\title{
Patients with rheumatoid arthritis facing sick leave or work disability meet varying regulations: a study among rheumatologists and patients from 44 European countries
} DOI:

10.1136/annrheumdis-2019-215294

\section{Document Version \\ Accepted author manuscript}

Link to publication record in Manchester Research Explorer

Citation for published version (APA):

Verstappen, S., \& et al. (2019). Patients with rheumatoid arthritis facing sick leave or work disability meet varying regulations: a study among rheumatologists and patients from 44 European countries. Annals of the rheumatic diseases. https://doi.org/10.1136/annrheumdis-2019-215294

\section{Published in:}

Annals of the rheumatic diseases

\section{Citing this paper}

Please note that where the full-text provided on Manchester Research Explorer is the Author Accepted Manuscript or Proof version this may differ from the final Published version. If citing, it is advised that you check and use the publisher's definitive version.

\section{General rights}

Copyright and moral rights for the publications made accessible in the Research Explorer are retained by the authors and/or other copyright owners and it is a condition of accessing publications that users recognise and abide by the legal requirements associated with these rights.

\section{Takedown policy}

If you believe that this document breaches copyright please refer to the University of Manchester's Takedown Procedures [http://man.ac.uk/04Y6Bo] or contact uml.scholarlycommunications@manchester.ac.uk providing relevant details, so we can investigate your claim.

\section{OPEN ACCESS}




\section{Annals of the \\ RHEUMATIC DISEASES}

The Eular Journal

\section{Patients with rheumatoid arthritis facing sick leave or work disability meet varying regulations: a study among rheumatologists and patients from 44 European countries}

\begin{tabular}{|c|c|}
\hline Journal: & Annals of the Rheumatic Diseases \\
\hline Manuscript ID & annrheumdis-2019-215294.R1 \\
\hline Article Type: & Extended report \\
\hline $\begin{array}{r}\text { Date Submitted by the } \\
\text { Author: }\end{array}$ & 01-Jul-2019 \\
\hline Complete List of Authors: & $\begin{array}{l}\text { Putrik, Polina; Maastricht University, Rheumatology } \\
\text { Ramiro, Sofia; Leiden University Medical Center, Rheumatology; } \\
\text { Zuyderland Medical Center, Rheumatology } \\
\text { Guillemin, Francis; School of Public Health, Medecine } \\
\text { Péntek, Márta; Corvinus University of Budapest, Department of Health } \\
\text { Economics; Flór Ferenc County Hospital, Rheumatology } \\
\text { Sivera, Francisca; Hospital Elda, Rheumatology Unit } \\
\text { Sokka, Tuulikki; Jyväskylä Central Hospital } \\
\text { de Wit, Maarten; Amsterdam University Medical Centre, Medical } \\
\text { Humanities } \\
\text { Woolf, Anthony; Rheumatology, } \\
\text { Zink, Angela; German Rheumatism Research Centre, Epidemiology } \\
\text { Andersone, Daina; Rheumatology } \\
\text { Berghea, Florian; Carol Davila University of Medicine and Pharmacy, } \\
\text { Butrimiene, Irena; Rheumatology Center, Vilnius University, State } \\
\text { Research Institute } \\
\text { Brouwer, Sandra; Groningen UMC, Department of Community and } \\
\text { Occupational Medicine } \\
\text { Cassar, Karen; University of Malta } \\
\text { Charalambous, Paraskevi; Cyrus Society for Rheumatology } \\
\text { Caporali, Roberto; University of Pavia, IRCCS Policlinico San Matteo } \\
\text { Foundation, Division of Rheumatology } \\
\text { Deseatnicova, Elena } \\
\text { Damjanov, Nemanja; University of Belgrade School of Medicine, } \\
\text { Rheumatology; Institute of Rheumatology, Director's office } \\
\text { Finckh, Axel; University of Geneva, Division of Rheumatology } \\
\text { FitzGerald, Oliver; St Vincents University Hospital } \\
\text { Grßndal, Ger ur; Landspitali University Hospital } \\
\text { Gobejishjvili, Nino; Health Center of Georgia } \\
\text { Głuszko, Piotr; Institute of Rheumatology, Clinic of Rheumatic Diseases } \\
\text { Hirsch, Marco } \\
\text { Jovanovic, Igor } \\
\text { Vencovský, Jiř́; Charles University } \\
\text { Janssens, Xavier } \\
\text { Keszei, Andras; Uniklinik RWTH Aachen University, Department of } \\
\text { Medical Informatics Uniklinik RWTH } \\
\text { Kovarova, Maria } \\
\text { Kull, Mart }\end{array}$ \\
\hline
\end{tabular}


Miranda, Luís; Portuguese Institute of Rheumatology, Mayer, Miroslav

Percinkova, Snezana

Inanc, Nevsun; marmara university, rheumatology

Nadashkevitz, Oleg

Petersson, Ingemar; Clinical Sciences Lund, Orthopedics

Puolakka, Kari; Lappeenranta Central Hospital, Department of Medicine Rojkovich, Bernadette; Department of Rheumatology, Semmelweis University, Polyclinic of the Hospitaller Brothers of St. John of God Radner, Helga; Medical University Vienna, Internal Medicine III; Departement of Rheumatology Szabados, Fruzsina

Slobodin, Gleb

Shirinsky, Ivan; Institute of Fundamental and Clinical Immunology, Laboratory of Clinical Immunopharmacology;

Soroka, Nikolay

Sidiropoulos, Prodromos; University of Crete School of Medicine, Rheumatology

Shumnalieva, Russka

Sokolovic, Sekib; Clinic of Heart and Rheumatic Diseases, Clinical Center University of Sarajevo, Rheumatology Department

Shukurova, Surayo

Tafaj, Argjend

Tomšič, Matija; University Medical Centre Ljubljana, Department of Rheumatology

Uhlig, Till; Diakonhjemmet Hospital, Rheumatology

Verstappen, Suzanne; University of Manchester, Arthritis Research UK Epidemiology Unit

Boonen, Annelies; Maastrich University Medical Center, Internal

Medicine, Division of Rheumatology

Keywords:

Epidemiology, Outcomes research, Rheumatoid Arthritis




Title:

\title{
Patients with rheumatoid arthritis facing sick leave or work disability meet varying regulations: a study among rheumatologists and patients from 44 European countries
}

\begin{abstract}
Authors:
Polina Putrik*1, Sofia Ramiro ${ }^{2}$, Francis Guillemin ${ }^{3}$, Marta Pentek ${ }^{4}$, Francisca Sivera ${ }^{5}$, Tuulikki Sokka ${ }^{6}$,Maarten de Wit ${ }^{7}$, Anthony Woolf ${ }^{8}$, Angela Zink ${ }^{9}$, Daina Andersone ${ }^{10}$, Florian Berghea ${ }^{11}$, Irena Butrimiene ${ }^{12}$, Sandra Brouwer ${ }^{13}$, Karen Cassar ${ }^{14}$, Paraskevi Charalambous $^{15}$, Roberto Caporali ${ }^{16}$, Elena Deseatnicova ${ }^{17}$, Nemanja Damjanov ${ }^{18}$, Axel Finckh ${ }^{19}$, Oliver FitzGerald ${ }^{20}$, Gerður Gröndal ${ }^{21}$, Nino Gobejishjvili22, Piotr Gluszko $^{23}$, Marco Hirsch ${ }^{24}$, Igor Jovanovic ${ }^{25}$, Jiri Vencovsky ${ }^{26}$, Xavier Janssens ${ }^{27}$, Andras Keszei ${ }^{28}$, Maria Kovarova ${ }^{29}$, Mart Kull ${ }^{30}$, Luis Cunha-Miranda ${ }^{31}$, Miroslav Mayer ${ }^{32}$, Snezana Misevska-Percinkova ${ }^{33}$, Inanc Nevsun ${ }^{34}$, Oleg Nadashkevich ${ }^{35}$, Ingemar F. Petersson ${ }^{36}$, Kari Poulakka ${ }^{37}$, B. Rojkovich ${ }^{38}$, Helga Radner ${ }^{39}$, Fruzsina Szabados ${ }^{40}$, Gleb Slobodin 41 , Ivan Shirinsky42, Nikolay Soroka ${ }^{43}$, Prodromos Sidiropoulos ${ }^{44}$, Russka Shumnalieva ${ }^{45}$, Sekib Sokolovic ${ }^{46}$, Surayo Shukurova ${ }^{47}$, Argiend Tafaj ${ }^{48}$, Matija Tomsic ${ }^{49}$, Till Uhlig ${ }^{50}$, Susanne Verstappen ${ }^{51}$, Annelies Boonen 1
\end{abstract}

*corresponding author: polina.putrik@gmail.com

\footnotetext{
${ }^{1}$ Rheumatology, MUMC, Maastricht, the Netherlands

${ }^{2}$ Rheumatology, Leiden University Medical Centre, Leiden, and Zuyderland Medical Center, Heerlen, the Netherlands

${ }^{3}$ University of Lorraine, APEMAC, Nancy, France

${ }^{4}$ Corvinus University, Budapest, Hungary

${ }^{5}$ Hospital General Universitario de Elda, Alicante, Spain

6 Jyväskylä Central Hospital, Jyväskylä, Finland

${ }^{7}$ Amsterdam University Medical Centre, Dept. Medical Humanities, Amsterdam Public Health (APH),

Amsterdam, Netherlands

${ }^{8}$ Royal Cornwall Hospital, Truro, United Kingdom

${ }^{9}$ German Rheumatism Research Centre and Rheumatology, Charité University Medicine, Berlin, Germany

${ }^{10}$ Rheumatology, Riga, Latvia

${ }^{11}$ Carol Davila University of Medicine, Bucharest, Romania

${ }^{12}$ Center of Rheumatology, Institute of Clinical Medicine, Medical Faculty, Vilnius University, Vilnius, Lithuania

${ }^{13}$ Department of Community and Occupational Medicine, University of Groningen, University Medical

Center Groningen, The Netherlands

${ }^{14}$ Faculty of Medicine and Surgery, University of Malta, Malta

${ }^{15}$ Cyrus Society for Rheumatology, Cyprus

${ }^{16}$ Division of Rheumatology, Universiry of Pavia and IRCCS S. Matteo Foundation, Pavia, Italy

17 Department of Rheumatology and Nephrology, State Medical and Pharmaceutical Universtiy

Nicolae Testemitanu, Republic of Moldova

${ }_{18}$ University of Belgrade School of Medicine, Institute of Rheumatology, Belgrade, Serbia

${ }^{19}$ Division of Rheumatology, University Hospital of Geneva (HUG), Switzerland

${ }^{20}$ Dept Rheumatology, St. Vincent's University Hospital and Conway Institute for Biomolecular

Research, University College Dublin. Ireland

${ }^{21}$ Centre for Rheumatology Research, University Hospital, Iceland
} 
22 Health Center of Georgia, Tbilisi, Georgia

${ }^{23}$ Department of Rheumatology, National Institute of Geriatrics, Rheumatology and

Rehabilitation, Warszawa, Poland

${ }^{24}$ Luxembourg, Luxembourg

25 Podgorica, Montenegro

${ }^{26}$ Institute of Rheumatology, Prague, Czech Republic

${ }^{27}$ Department of Rheumatology, Ghent University Hospital, Ghent, Belgium

${ }^{28}$ Medical Informatics, Uniklinik RWTH Aachen University, Aachen, Germany

29 Slovakia, St.CM University, Trnava, Slovakia

30 Tartu University, Department of Rheumatology and Internal Medicine and Viljandi Hospital, Tartu,

Estonia

31 Instituto Português de Reumatologia, Lisbon, Portugal

32 Division of Clinical Immunology and Rheumatology, Department of Internal Medicine, University Hospital Centre Zagreb and University of Zagreb, School of Medicine, Zagreb, Croatia

33 University Rheumatology Clinic, Medical Faculty, Former Yugoslav Republic of Macedonia

(FYROM)

${ }^{34}$ Division of Rheumatology, School of Medicine, Marmara University, Istanbul, Turkey

${ }^{35}$ Lviv National Medical University, Lviv, Ukraine

${ }^{36}$ Skåne University Hospital, Lund, Sweden and Clinical Sciences Lund, Lund University, Lund

Sweden

${ }^{37}$ South Karelia Central Hospital, Finland

${ }^{38}$ Polyclinic of the Hospitaller Brothers of St. John of God, Budapest, Hungary

39 Department of Internal Medicine III, Division of Rheumatology, Medical University Vienna, Vienna,

Austria

${ }^{40}$ Department of Rheumatology, North Denmark Regional Hospital

${ }^{41}$ Rheumatology Unit, Bnai Zion Medical Center, Haifa, Israel

${ }^{42}$ Laboratory of clinical immunopharmacology, Clinical immunology institute, Novosibirsk, Russia

${ }^{43}$ Rheumatology, Minsk, Belarus

${ }^{44}$ Greece, Medical School, University of Crete, Greece

${ }^{45}$ Clinic of Rheumatology, University Hospital "St. Ivan Rilski", Department of Internal

Medicine, Medical University -Sofia, Bulgaria

46 Sarajevo, Bosnia\&Herzegovina

${ }^{47}$ Medical Science Academy, Tajikistan, Dushanbe

48 Medical University Of Tirana, Rheumatology clinic, "Nene Tereza" Hospital, Tirane, Albania.

${ }^{49}$ Department of Rheumatology, University Medical Centre, Ljubljana, Slovenia

${ }^{50}$ Diakonhjemmet Hospital, Oslo, Norway

${ }^{51}$ Arthritis Research UK Centre for Epidemiology, Division of Musculoskeletal \& Dermatological Sciences, School of Biological Sciences, Faculty of Biology, Medicine and Health, The University of Manchester, Manchester Academic Health Science Centre, Manchester, United Kingdom; NIHR Manchester Biomedical Research Centre, Central Manchester University Hospitals NHS Foundation Trust, Manchester Academic Health Science Centre, United Kingdom; Arthritis Research UK-MRC Centre for Musculoskeletal Health and Work, University of Southampton, United kingdom.

\section{Word count: 3,690}

\section{Working group:}

Ermir Tafaj (Albania), Edi Rembeci (Albania), Saskia Decuman (Belgium), Anastasia Tushina (Belarus), Iva Miteva (Bulgaria), Šárka Forejtová (Czech Republic), Esben Toftegaard Knudsen (Denmark), Maxime Wach (France), Matthieu Mangin (France), Márta Fekete (Hungary), Antonella Celano (Italy), Grainne O'Leary (Ireland), Giedre Dereseviciene (Lithuania), Mona Thorekildsen (Norway), Leszek Roszkowski (Poland), Claudia Handra (Romania), Inessa Samko (Russia), Jenny Hubertsson (Sweden), Tapparel Ludovic (Switzerland), Zumrad Hamroeva(Tajikistan), Ilker Yagcı (Turkey), Ana Zekovic (Serbia), James Anderson (UK)

\section{Abstract}




\section{Objectives}

To describe and explore differences in formal regulations around sick leave and work disability (WD) for patients with rheumatoid arthritis (RA), as well as perceptions by rheumatologists and patients on the system's performance, across European countries.

\section{Methods}

We conducted three cross-sectional surveys in 50 European countries: one on work (re-)integration and social security (SS) system arrangements in case of sick leave and long-term WD due to RA (one rheumatologist per country), and two among approximately 15 rheumatologists and 15 patients per country on perceptions regarding SS arrangements on work participation. Differences in regulations and perceptions were compared across categories defined by Gross Domestic Product (GDP), type of social welfare regime, European Union (EU) membership and country RA WD rates.

\section{Results}

Forty-four (88\%) countries provided data on regulations, 33(75\%) on perceptions of rheumatologists $(n=539)$, and $34(77 \%)$ on perceptions of patients $(n=719)$. While large variation was observed across all regulations across countries, no relationship was found between most of regulations or income compensation and GDP, type of SS system, or rates of WD. Regarding perceptions, rheumatologists in high GDP and EU-member countries felt less confident in their role in the decision process towards WD $(\beta=-0.5[95 \% \mathrm{Cl}[-0.9 ;-0.2]$ and $\beta=-0.5[-1.0 ;-0.1]$, respectively). The Scandinavian and Bismarkian system scored best on patients' and rheumatologists' perceptions of regulations and system performance.

\section{Conclusions}

There is large heterogeneity in rules and regulations of SS systems across Europe in relation to WD of patients with $R A$, and it cannot be explained by existing welfare regimes, EU membership or country's wealth. 


\section{Introduction}

Rheumatoid arthritis (RA) is a chronic inflammatory joint disease that often starts during working life of patients.[1-3] RA has a high impact on functioning and ability to participate in social roles.[4, 5] Despite substantial improvements in treatment options in the last decades, still $20 \%$ of the patients are unable to continue to work in the first 3 years of disease and over $30 \%$ of patients become work disabled after 10 years.[6-9]

For patients, work disability (WD) implies exclusion from an essential role in society, but also loss of income and reduced economic self-sufficiency.[5, 10] To prevent individuals from poverty in case of WD, substitution of income has been introduced in some European countries in the $19^{\text {th }}$ century. By now, most countries have some form of social security (SS) system in place to regulate income substitution. However, these systems are not uniform, as they have been shaped by national political and social developments throughout the $20^{\text {th }}$ century. Policies targeted at income substitution balance between provision of a fair income, on one hand, and control of expenditures by restricting social security benefits only to individuals with specific levels of work restrictions, on the other hand.[11] More recently, the increasing economic burden of WD, but also the insight into social and health benefits of work participation stimulated in many countries stricter gate-keeping on the one hand, and stronger employment support to enhance endurable work participation of persons with chronic disease on the other hand.[12]

SS arrangements have been suggested to result in differences in overall patterns of employment, WD, and retirement.[13, 14] A recent worldwide multinational study (COMORA) has shown that lower economic wealth and human development of countries is associated with higher rates of unemployment and higher absenteeism.[15] Earlier, QUEST RA study has observed that in low Gross Domestic Product (GDP) countries, people remain working with higher levels of disability and disease activity compared to high GDP countries.[16] The regulations around the sick leave and work disability may be, at least partially, responsible for these differences, however, they have not been studied.

Policies with regard to work participation, however, go far beyond income substitution only. Criteria for access to WD benefits as well as levels of income 
substitution likely depend, among others, on economic, political and cultural factors. While the European Union (EU) and World Health Organization (WHO) accept the historical differences in the way health and social systems are organised and function in their member states, there is a universal agreement that differences should not result in inequalities in health and quality of life of people across nations.[17] It is unclear whether EU member states achieved any degree of homogeneity in the key regulations around social policies with regards to work disability. In addition to system level factors, there is evidence that personal contextual and disease related factors [6, 18, 19], influence the decision of an individual to take sick leave or apply for long term WD.

The aim of this study was to describe and explore differences in formal regulations around sick leave and WD for patients with RA, as well as perceptions by rheumatologists and patients on the system's performance, across European countries. We hypothesised that 1) lower GDP countries have stricter rules with regard to obtaining work disability and lower income substitution once work disability is granted 2) EU countries have more homogeneous regulations compared to nonEU countries 3) patients and rheumatologists in high GDP and EU member states are more satisfied with the performance of the social security system.

\section{Methods}

\section{Design and framework}

We conducted a cross-sectional observational study consisting of three surveys in 50 of the 53 countries of the European WHO Region (in three countries, no contact person could be found), in 2014-2016 (Figure 1). The questionnaires were designed following the framework of access (originally applied in healthcare $[20,21]$ ), with three dimensions : i) availability of re-integration plans or other systems/policies to facilitate work and/or prevent WD, as well as the eligibility criteria that a patient should meet to receive SS benefits; ii) affordability, i.e. the level of income substitution granted in case of sick leave and permanent disability; iii) acceptability, i.e. professional and individual perceptions of rheumatologists and patients, 
respectively, around the system performance on these issues. The study has been approved by Maastricht Ethical Committee.

\section{Participants}

For each of the 50 European countries, one rheumatologist was invited as principal investigator $(\mathrm{PI})$ to complete the questionnaire on SS arrangements in his/her country in case of sick leave and long-term WD due to RA (survey 1; availability and affordability). Additionally, each PI was asked to invite at least 15 rheumatologists (survey 2; acceptability) and at least 15 patients (survey 3; acceptability) to complete a questionnaire on professional and individual perceptions of the system. To recruit rheumatologists, Pls were instructed to ensure a diverse sample in terms of gender, years of professional experience, and clinical setting. Patients could also be recruited via patient organizations and aimed at representing the spectrum of patients with RA, assuring that at least a half of them had experience with (applying for a) WD pension.

\section{Questionnaires}

The questionnaire for Pls addressed national regulations (in 2014) on benefits separately for sick leave and WD (Online Supplementary Text S1a: availability and affordability), as well as calculations of the level of income for nine pre-specified scenarios (vignettes), one on sick leave and two for long-term WD across the three levels of income (Online Supplementary Text S1b). In countries where a patient research partner was available $(n=21)$, he/she was invited to comment on any inconsistencies in the answers on the main questionnaire about the formal regulations. In this case, answers were double-checked with the PI.

The questionnaire for rheumatologists (Online Supplementary Text S2; acceptability) contained questions about perceptions on appropriateness of the SS arrangements, practical aspects of the application process for benefits, and the role of rheumatologists in the process. The questionnaire for patients (Online Supplementary Text S3; acceptability) addressed perceptions about the importance and adequacy of the existing arrangements. Additional questions were included on age, gender, work environment (non-university hospital, university hospital, private practice, other) for rheumatologists; on age, gender, disease duration, work status (paid work, no paid work but not work disabled, partially or fully work disabled) and 
history of sick leave and WD. The PI decided on whether questionnaires could be applied in English, otherwise translated them, wherever possible with patient partners involved in checking the translation.

Data on Gross Domestic Product (GDP) per capita (in international dollars, 2013) were extracted from the World Development Indicators report by the World Bank [22] and used as a continuous variable or dichotomized around the median (27,000 int.\$). The welfare regimes taxonomy included five groups, namely the Anglo-Saxon, Bismarckian, Mediterranean, Post-Communist, and Scandinavian type of system.[23] Rates of work disability among RA patients have been collected by the QUEST-RA study (2009) and were available for 21 countries from our sample.[16, 24]

\section{Statistical analysis}

Arrangements to support work and social security regulations in case of sick leave or work disability due to RA (questionnaire 1)

Collected data on formal rules and policies were first presented through descriptive statistics. To investigate whether the regulations differed by the type of welfare regime[23], GDP, EU membership (EU-15, new EU member states and non-EU countries), or were associated with country-level work disability rates among RA patients[16], subgroup comparisons were performed using Pearson correlations, ttest/Mann-Whitney and chi-square/Fisher's test, as appropriate.

Patients' and rheumatologists' perceptions of the social security system (questionnaires 2 and 3)

Answers of rheumatologists on their perspective of SS arrangements were summarized and scored (the higher is the better) around the two domains: (1) 'Performance of the system' (score 0-4) and (2) 'Role of the rheumatologists' (score $0-4)$. Additionally, a single item on the perceived standardisation in the decisionmaking process was analysed separately. Input from patients was summarized following three domains: (1) 'Importance and support to remain employed' (score 05), (2) 'Process of applying for WD (score 0-4), and (3) 'Obtaining and living with work disability pension' (score 0-6) (complete questionnaires are provided in the Online Supplementary Texts S2 and S3). Each domain consisted of 4 to 6 questions (each on a 1 (totally agree) to 5 (totally disagree) Likert scale, dichotomized as 1 ('(totally) agree') and 0 ('not agree/not disagree', '(totally) disagree'). The 
dichotomized scores per question were summed into the five domain scores (two for rheumatologists and three for patients).

Rheumatologists' and patients' characteristics were compared across the type of SS system, EU membership, and GDP. Small numbers of surveyed patients and rheumatologists in each country hindered analyses of country level means, and thus were not related to national RA work disability rates. The domain 'Importance and support to remain employed' was assessed in patients currently or ever having worked. Analyses in domains 'process of applying for work disability and 'obtaining and living with work disability were limited to patients currently work disabled or ever considered applying for WD.

Finally, we conducted multilevel (with individuals clustered in countries) multiple regression analysis with each of the domains as an outcome and type of SS system, EU membership or GDP as the independent variable of interest. Models with patient perceptions as outcome were adjusted for age, gender, education, disease duration and ever having had sick leave due to RA. When rheumatologists' perceptions were the outcome, analyses were adjusted for age, gender and work setting.

\section{Results}

Forty-four (28 EU and 16 non-EU member states) countries (88\%) provided data on formal rules and regulations for sick leave and WD. Of these, 33 (75\%) countries collected data from rheumatologists $(n=539)$, and $34(77 \%)$ countries collected data from patients $(n=719)$ (missing countries were all non-EU members except Luxemburg).

Arrangements to support work and social security regulations in case of sick leave of work disability due to RA

While nearly all countries had arrangements to support patients with restrictions to work, a large heterogeneity was observed in the type of arrangements (Table 1, Online Supplementary Tables S1-3). All except for 12 countries had facilities to support patients with RA in paid employment $(n=32,73 \%)$, but only in a quarter of countries $(n=11,25 \%)$ rehabilitation efforts were obligatory prior to the decision about long-term WD. Twenty-five (57\%) and 30 (68\%) countries had a requirement for employment history or social insurance contributions in order to be eligible for sick leave or long-term WD compensation, respectively. The maximum sick leave 
length before transition to long-term WD varied from 3 to 36 months (mean (SD) 13 (9)). In $8(18 \%)$ and $5(11 \%)$ of the countries, participation of a rheumatologist was mandatory in the process of application or decision-making process on long-term WD, respectively. In addition to a functional assessment (degree of (dis)ability), prior profession, $(n=25(57 \%)$ of countries), diagnosis $(n=32(73 \%))$, earning capacity $(n=12(27 \%))$, age $(n=20(45 \%))$, and gender $(n=5(11 \%))$ were reported to be accounted for when a decision was taken about (the degree of) WD. Prognosis, education and place of residence were mentioned as additional factors by few countries.

All countries except the former Yugoslav Republic of Macedonia, BosniaHerzegovina and Serbia reported to recognise partial WD, a status that partially substitutes income while the person can continue in (reduced) employment. Of the 26 countries providing data on income substitution, in 8 countries (31\%) income substitution (averaged over the first 6 months of disability) in case of sick leave was less than $70 \%$ of previous income. Income substitution averaged over the first 12 months of disability was less than $70 \%$ in $18(70 \%)$ and $15(58 \%)$ in case of moderate (partial) or severe (full) long-term disability, respectively (Table 2). While wealthier countries as expected provided higher benefits in absolute terms, when converted to percentage of the previously earned income, no relationship was found between income compensation and neither GDP, nor the type of SS system, or rates of work disability. In richer countries and in countries with the Bismarkian type of welfare regime, the WD pension burden was more likely to be shared between SS and a private insurance, while countries with lower GDP and other welfare regimes had social insurance as the main source of WD allowances (data not shown). In countries with lower GDP, a rheumatologist was more frequently necessarily involved in the application and decision making. Other aspects of the system revealed no statistically significant patterns with country-level characteristics or national WD rates.

\section{Rheumatologists' and patients' and perceptions of social security system}

In total, 539 rheumatologists (mean age (SD) 48 (10), 284 (53\%) female, 278 (51\%) working in university hospitals) from 33 countries filled in the questionnaires (Online Supplementary Table S4). Scores on 'Role of the rheumatologists' (0-4) and 'Performance of the system' (0-4) ranged from 1.4 (SD 0.9) (Anglo-Saxon) to 2.4 (1.1) (Post-communist) and 0.8 (0.9) (Anglo-Saxon) to 2.3 (1.2) (Scandinavian), 
respectively. Perceived level of standardization around decision-taking revealed that only $26 \%$ ( $n=135)$ of rheumatologists consider decisions on WD to be objective. Of note, those who perceived standardization as poor (vs good) scored worse on both 'Role of the rheumatologists' (-0.4 points) and 'Performance of the system' (-2.6 points) domains (t-test $p$-value for both scores $<0.05$ ). Multilevel analyses revealed that rheumatologists in high GDP (vs low GDP) and EU-member (vs non-EU member) countries felt less confident in having an active role in WD decisions ( $\beta=-$ $0.5[95 \% \mathrm{Cl}[-0.9 ;-0.2]$ and $\beta=-0.5[-1.0 ;-0.1]$, respectively). In addition, significant differences were observed across the system types with the Scandinavian type (Denmark, Iceland, Sweden, Norway, Finland) consistently scoring higher than the others on domains 'Importance and support to remain employed' and 'Process of applying for work disability pension' (Table 4 and Supplementary Table S5).

The patient sample consisted of 719 patients from 34 countries (mean age (SD) 53 (12), $76 \%$ female, 519 (78\%) ever worked). The highest (=most satisfied) patient scores on all three domains were consistently observed in countries with Scandinavian and Bismarkian type of security system (Table 3). In multilevel adjusted regression models, neither country wealth nor EU status were associated with patients' perceptions (Table 4 and Supplementary Table S5). The findings across the system type were notably consistent across patient and rheumatologist domains. 
Table 1. Summary of social security regulations to support stay at work and work disability arrangements for persons with RA

\begin{tabular}{|c|c|c|c|c|c|c|c|}
\hline \multicolumn{2}{|l|}{ Regulation } & $\mathbf{N}(\%)^{*}$ & $\begin{array}{l}\text { Anglo- } \\
\text { saxon }\end{array}$ & Scandinavian & Bismarkian & Mediterranean & Post-communist \\
\hline \multicolumn{8}{|l|}{ Support to stay at work } \\
\hline \multirow{2}{*}{$\begin{array}{l}\text { Facilities for persons with RA } \\
\text { that aimed at keeping the } \\
\text { patient in paid employment are } \\
\text { available }\end{array}$} & Yes & $32(73 \%)$ & $\underline{\text { IEE}}, \underline{\mathrm{GB}}$ & $\frac{\mathrm{DK}}{\mathrm{SE}}, \underline{\mathrm{FI}}, \underline{\mathrm{IS}}, \underline{\mathrm{NO}}$, & $\begin{array}{l}\text { AU, }, \underline{B E}, \underline{\mathrm{DE}}, \\
\mathrm{NL}, \underline{\mathrm{LU}}, \underline{\mathrm{CH}},\end{array}$ & $\underline{\mathrm{CY}}, \underline{\mathrm{II}}, \underline{\mathrm{PT}}$ & $\begin{array}{l}\text { AL, BY, BG, CR, CZ, LT, } \\
\text { MK, MD, ME, PL, RO, } \\
\text { RS, SK, SL, TJ }\end{array}$ \\
\hline & No & $12(27 \%)$ & - & - & FR & $\begin{array}{l}\text { GR, MT, ES, } \\
\text { TR }\end{array}$ & $\begin{array}{l}B^{B P} E^{P} E^{P} G^{P} H^{P} R U^{P} \\
\text { LV }^{P} \cup A^{P}\end{array}$ \\
\hline \multicolumn{8}{|c|}{ Access to sick-leave or short term absence } \\
\hline \multirow{2}{*}{$\begin{array}{l}\text { Requirement for length of } \\
\text { previous employment AND/OR } \\
\text { certain income earned } \\
\text { AND/OR certain amount of } \\
\text { social insurance tax paid in } \\
\text { order to be eligible for sick } \\
\text { leave compensation }\end{array}$} & Yes & $25(57 \%)$ & $\underline{\text { IEE, }} \underline{\mathbf{G B}}$ & DK, NO, SE & $\frac{\mathrm{BE}}{\mathrm{CH}}, \underline{\mathrm{FR}}, \underline{\mathrm{IL}}$, & $\begin{array}{l}\text { CY, GR, PT, } \\
\text { ES }\end{array}$ & $\begin{array}{l}\text { AL, BY, BA, BG, HR, } \\
\text { CZ, LT, MD, PL, RO, } \\
\text { SK, TJ } \\
\end{array}$ \\
\hline & No & $18(41 \%)$ & & FI, IS & $\frac{\mathrm{AU}}{\mathrm{NL}}, \underline{\mathrm{DE}}, \underline{\mathrm{LU}}$, & IT, MT, TR & $\begin{array}{l}\text { EE, GE, HU, LV, MK, } \\
\text { ME, RU, RS, SL, UA }\end{array}$ \\
\hline \multirow{2}{*}{$\begin{array}{l}\text { The rheumatologist is } \\
\text { authorized to certify short-term } \\
\text { absence from work due to RA }\end{array}$} & Yes & $33(75 \%)$ & $\underline{\text { IE }}$ & $\frac{\mathrm{DK}}{\mathrm{SE}}, \underline{\mathrm{FI}}, \underline{\mathrm{IS}}, \underline{\mathrm{NO}}$, & 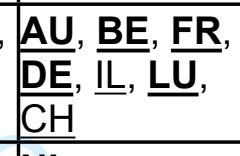 & $\begin{array}{l}\text { CY, GR, MT, } \\
\text { PT, ES, TR }\end{array}$ & $\begin{array}{l}\text { AL, BY, BA, CZ, EE, } \\
\text { HU, LT, MK, ME, PL, } \\
\text { RO, RU, TJ, UA }\end{array}$ \\
\hline & No & $10(23 \%)$ & $\underline{\text { GB }}$ & & $\mathrm{NL}$ & IT & $\begin{array}{l}\text { BG, CR, GE, LV, MD, } \\
\text { RS, SK, SL }\end{array}$ \\
\hline \multirow[b]{2}{*}{$\begin{array}{l}\text { First } 1-3 \text { days of sick leave are } \\
\text { unpaid }\end{array}$} & Yes & $11(25 \%)$ & - & SE & FR, IL & $\frac{\mathbf{C Y}}{\mathrm{TR}}, \underline{\mathbf{I T}}, \underline{\mathbf{P T}}, \underline{\mathbf{E S}}$, & CZ, EE, LV \\
\hline & No & $32(73 \%)$ & $\underline{\text { IEE }}, \underline{\mathbf{G B}}$ & $\underline{D K}, \underline{\mathrm{FI}}, \mathrm{IS}, \underline{\mathrm{NO}}$ & $\frac{\mathrm{AU}}{\mathrm{LU}}, \underline{\mathrm{BE}}, \underline{\mathrm{CH}}, \underline{\mathrm{NL}}$ & GR, $\underline{\mathrm{MT}}$ & $\begin{array}{l}\text { AL, BY, BA, BG, CR, } \\
\text { GE, HU, LT, MK, MD, } \\
\text { ME, PL, RO, RU, RS, } \\
\text { SK, SL, TJ, UA }\end{array}$ \\
\hline \multicolumn{8}{|c|}{ Decision about long-term work disability } \\
\hline \multirow[t]{2}{*}{$\begin{array}{l}\text { Maximum length of sick leave } \\
\text { before a WD can be granted }\end{array}$} & $\begin{array}{l}<12 \\
\text { months }\end{array}$ & $35(80 \%)$ & $\underline{\text { GB }}$ & DK, IS, $\underline{\text { SE }}$ & AU, & $\begin{array}{l}\text { CYY, GR, IT, } \\
\text { MT, PT, ES, }\end{array}$ & $\begin{array}{l}\text { AL, BY, BG, CR, EE, } \\
\text { LV, LT, HU, MK, MD, } \\
\text { ME, PL, RO, RU, RS, } \\
\text { SL, TJ, UA }\end{array}$ \\
\hline & $\geq 12$ & $8(18 \%)$ & $\underline{\underline{I E}}$ & FI, NO & $\mathrm{BE}, \underline{\mathrm{CH}}, \underline{\mathrm{NL}}$ & - & BA, $\underline{\mathbf{C Z}}, \mathbf{S K}$ \\
\hline
\end{tabular}




\begin{tabular}{|c|c|c|c|c|c|c|c|}
\hline \multicolumn{8}{|c|}{ Annals of the Rheumatic Diseases } \\
\hline & months & & & & & & \\
\hline \multirow{2}{*}{$\begin{array}{l}\text { Is it obligatory that a } \\
\text { rheumatologist is involved in } \\
\text { application process (i.e. } \\
\text { providing information about the } \\
\text { disease)? }\end{array}$} & Yes & $10(23 \%)$ & - & - & - & CY, GR & $\begin{array}{l}\text { AL, BY, BG, LT, } \\
\text { MK,SK,RU, TJ }\end{array}$ \\
\hline & No & $31(70 \%)$ & $\underline{\underline{\mathrm{IE}}, \underline{\mathrm{GB}}}$ & DK, FI, NO & $\begin{array}{l}\mathrm{AU}, \underline{\mathrm{BE}}, \underline{\mathrm{FR}}, \\
\mathrm{IL}, \underline{\mathrm{LU}}, \underline{\mathrm{CH}}, \\
\mathrm{DE}\end{array}$ & IT, $, \frac{M T}{\mathrm{ES}}, \underline{\mathrm{PT}}$, & $\begin{array}{l}\text { BA, CR, CZ, EE, HU, LV } \\
\text {, MD, PL, RO, RS, } \underline{\text { SL, }} \\
\text { UA }\end{array}$ \\
\hline \multirow[b]{2}{*}{$\begin{array}{l}\text { Is the rheumatologist also in } \\
\text { an obligatory way involved in } \\
\text { the decision-taking about the } \\
\text { long-term work disability? (i.e. } \\
\text { is part of the commission or } \\
\text { expert committee) } \xi\end{array}$} & Yes & $5(11 \%)$ & - & - & - & CY & AL, BG, SK, TJ \\
\hline & No & $36(82 \%)$ & $\underline{\underline{\mathrm{IE}}, \underline{\mathrm{GB}}}$ & DK, FI, NO & $\begin{array}{l}\mathrm{AU}, \mathrm{BE}, \frac{\mathrm{FR}}{}, \\
\mathrm{IL}, \mathrm{LU}, \underline{\mathrm{CH}}, \\
\mathrm{NL}, \mathrm{DE}\end{array}$ & $\begin{array}{l}\text { GR, IT, MT, } \\
\text { PT, ES, }\end{array}$ & $\begin{array}{l}\text { BY, BA, CR, CZ, EE, } \\
\text { HU, LV, LT, MK, MD, } \\
\text { PL, RO, RU, RS, SL, UA }\end{array}$ \\
\hline \multirow{2}{*}{$\begin{array}{l}\text { Rehabilitation efforts are } \\
\text { obligatory before a patient } \\
\text { could be assessed for long- } \\
\text { term disability }\end{array}$} & Yes & $11(25 \%)$ & & $\underline{\mathrm{DK}}, \underline{\mathrm{FI}}, \underline{\mathrm{SE}}, \underline{\mathrm{NO}}$ & $\mathrm{AU}, \underline{\mathrm{CH}}, \underline{\mathrm{NL}}$ & 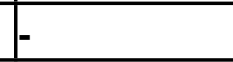 & LT, MD, ME, RO, SL \\
\hline & No & $32(73 \%)$ & IE, GB & IS & 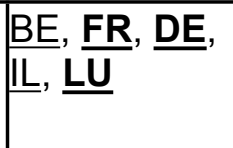 & $\begin{array}{l}\text { CYY, GR, IT, } \\
\text { MT, PT, ES, } \\
T R\end{array}$ & $\begin{array}{l}\text { AL, BY, BA, BG, CR, } \\
\text { CZ, EE, HU, LV, MK, } \\
\text { PL, RU, RS, SK, TJ, UA }\end{array}$ \\
\hline
\end{tabular}

AL, Albania; AT, Austria; BA, Bosnia and Herzegovina; BE, Belgium; BG, Bulgaria; BY, Belarus; HR, Croatia, CH, Switzerland; CY, Cyprus; CZ, Czech Republic; DE, Germany; GE, Georgia; DK, Denmark; EE, Estonia; ES, Spain; FI, Finland; FR, France; GR, Greece; HU, Hungary; HR, Croatia; IE, Ireland; IS, Iceland; IL, Israel; IT, Italy; LT, Lithuania; LU, Luxemburg; LV, Latvia; MD, Moldova; ME, Montenegro; MK, Macedonia (the former Yugoslav Republic of Macedonia); MT, Malta; NL, The Netherlands; NO, Norway; PL, Poland; RO, Romania; RU, Russia; SE, Sweden; SK, Slovakia; SL, Slovenia; RS, Serbia; TR, Turkey; TJ, Tajikistan, UK, United Kingdom; UA, Ukraine

*data provided by 44 countries, Georgia reported to not have any effective social security system in place; $\xi$ : data missing for IS, ME

EU countries are in bold, High GDP ( $>27,000$ int. dollars, based on median) countries are underscored 
Table 2. Income compensation in case of work disability for an employee with average income*

\begin{tabular}{|c|c|c|c|c|c|c|c|}
\hline \multirow{2}{*}{$\begin{array}{l}\begin{array}{l}\text { Type of } \\
\text { disability }\end{array} \\
\text { In case of sick } \\
\text { leave }\end{array}$} & \multicolumn{2}{|c|}{ Income compensation } & \multirow{2}{*}{\begin{tabular}{|l|} 
Anglo-saxon \\
IE \\
\end{tabular}} & \multirow[t]{2}{*}{ Scandinavian } & \multirow{2}{*}{\begin{tabular}{|l|} 
Bismarkian \\
$\mathrm{BE}$
\end{tabular}} & \multirow{2}{*}{\begin{tabular}{|l|} 
Mediterranean \\
CY, PT, TR \\
\end{tabular}} & \multirow{2}{*}{\begin{tabular}{|l|}
$\begin{array}{l}\text { Post- } \\
\text { communist }\end{array}$ \\
CZ, EE, SK \\
\end{tabular}} \\
\hline & $\begin{array}{l}\leq 70 \% \text { of earned } \\
\text { income }\end{array}$ & $8(31 \%)^{\star *}$ & & & & & \\
\hline & $\begin{array}{l}>70 \% \text { of earned } \\
\text { income }\end{array}$ & $18(69 \%)$ & - & FI, NO & $\underline{L U}, \underline{\mathrm{NL}}, \underline{\mathrm{SE}}$ & FR, IT, ES & $\begin{array}{l}\text { BG, LT, LV, } \\
\text { MD, PL, RO, } \\
\text { RS, SL }\end{array}$ \\
\hline \multirow[t]{2}{*}{$\begin{array}{l}\text { In case of } \\
\text { moderate }(50 \%) \\
\text { work disability* }\end{array}$} & $\begin{array}{l}\leq 70 \% \text { of earned } \\
\text { income }\end{array}$ & $18(70 \%)$ & $\underline{\text { IE }}$ & FI, NO & $\mathrm{BE}, \underline{\mathrm{SE}}, \underline{\mathrm{CH}}$ & CY, TR & $\begin{array}{l}\text { BY, BG, CZ, } \\
\text { EE, LV, LT, } \\
\text { MD, PL, RO, } \\
\text { RS, SK }\end{array}$ \\
\hline & $\begin{array}{l}>70 \% \text { of earned } \\
\text { income }\end{array}$ & $7(27 \%)$ & - & & $\underline{L U}, \underline{\mathrm{NL}}$ & FR, IT, PT, ES & $A L$ \\
\hline \multirow[t]{2}{*}{$\begin{array}{l}\text { In case of } \\
\text { severe }(75 \%) \\
\text { work disability* }\end{array}$} & $\begin{array}{l}\leq 70 \% \text { of earned } \\
\text { income }\end{array}$ & $15(58 \%)$ & & FI, NO & $\mathrm{BE}, \underline{\mathrm{LU}}, \underline{\mathrm{SE}}, \mathrm{CH}$ & CY, & \begin{tabular}{|l} 
CZ, EE, LV, LT, \\
MD, PL, RO, \\
RS, SK
\end{tabular} \\
\hline & $\begin{array}{l}>70 \% \text { of earned } \\
\text { income }\end{array}$ & $10(38 \%)$ & IE & & $\mathrm{NL}$ & FR, II, PT, ES, & $A L, B Y, B G$ \\
\hline
\end{tabular}

AL, Albania; BY, Belarus, BE; Belgium; BG; CY, Cyprus; CZ, Czech Republic; EE, Estonia; FI, Finland; FR, France; IE, Ireland; IT, Italy; LV, Latvia; LT, Lithuania;

LU, Luxemburg; MD, Moldova; NO, Norway; PL - Poland; PT, Portugal; RO, Romania; RS, Serbia; SK, Slovakia; SL, Slovenia; ES, Spain; CH, Switzerland; NL, the Netherlands; TR, Turkey

EU countries are in bold, High GDP ( $>27,000$ int. dollars, based on median) countries are underscored ${ }^{*}$ SL did not provide data for long term WD

*a person 50 y.o. recently diagnosed with RA, who is a citizen and has worked for 25 years full-time

${ }^{* *}$ data provided by 26 countries 
Table 3. Patients' and rheumatologists' characteristics per type of security system.

\begin{tabular}{|c|c|c|c|c|c|c|c|}
\hline & Total & Anglo-saxon & Scandinavian & Bismarkian & Mediterranean & Post-communist & p-value \\
\hline \multicolumn{8}{|c|}{ Rheumatologists characteristics (Questionnaire 2) } \\
\hline $\mathrm{N}$ of rheumatologists ( $\mathrm{N}$ of countries) & $539(33)$ & $22(2)$ & $58(5)$ & $88(6)$ & $87(6)$ & $284(15)$ & \\
\hline Role of the rheumatologists $(0-4)$ & $2.1(1.1)$ & $1.4(0.9)$ & $1.3(0.9)$ & $2.1(1.3)$ & $1.9(1.0)$ & $2.4(1.1)$ & $<0.001$ \\
\hline Performance of the system (0-4) & $1.5(1.1)$ & $0.8(0.9)$ & $2.3(1.2)$ & $2.0(1.1)$ & $1.1(1.0)$ & $1.4(1.1)$ & $<0.001$ \\
\hline \multicolumn{8}{|l|}{ Patient characteristics (Questionnaire 3) } \\
\hline $\begin{array}{l}\text { Importance and support to remain employed } \\
(0-5)\end{array}$ & $2.1(1.2)$ & $1.6(0.8)$ & $2.6(1.2)$ & $2.3(1.4)$ & $2.1(1.2)$ & $2.0(1.1)$ & $<0.001$ \\
\hline Process of applying for WD (0-4) & $2.2(1.8)$ & $1.3(1.8)$ & $3.2(3.0)$ & $2.4(1.9)$ & $2.0(1.6)$ & $2.3(1.7)$ & $<0.001$ \\
\hline Obtaining and living with WD pension $(0-6)$ & $1.1(1.1)$ & $0.7(0.8)$ & $1.2(1.1)$ & $1.2(1.4)$ & $1.0(1.1)$ & $1.1(1.1)$ & 0.22 \\
\hline
\end{tabular}

Anglo-saxon: UK, Ireland; Scandinavian: Denmark, Iceland, Sweden, Norway, Finland; Bismarkian: Austria, Belgium, Germany, France, Israel, Netherlands, Switzerland; Mediterranean: Cyprus, Greece, Italy, Portugal, Spain, Turkey; Post-communist: Albania, Bulgaria, Czech Republic, Croatia, Estonia, Georgia, Hungary, Latvia, Lithuania,

Poland, Romania, Russian Federation, Serbia, Tajikistan, Slovak Republic, Slovenia

Table 4. Patients' and rheumatologists' perceptions across several domains according to (1) GDP per capita PPP (2) EU membership status (3) Type of social security system. Models adjusted for socio-demographic confounders.

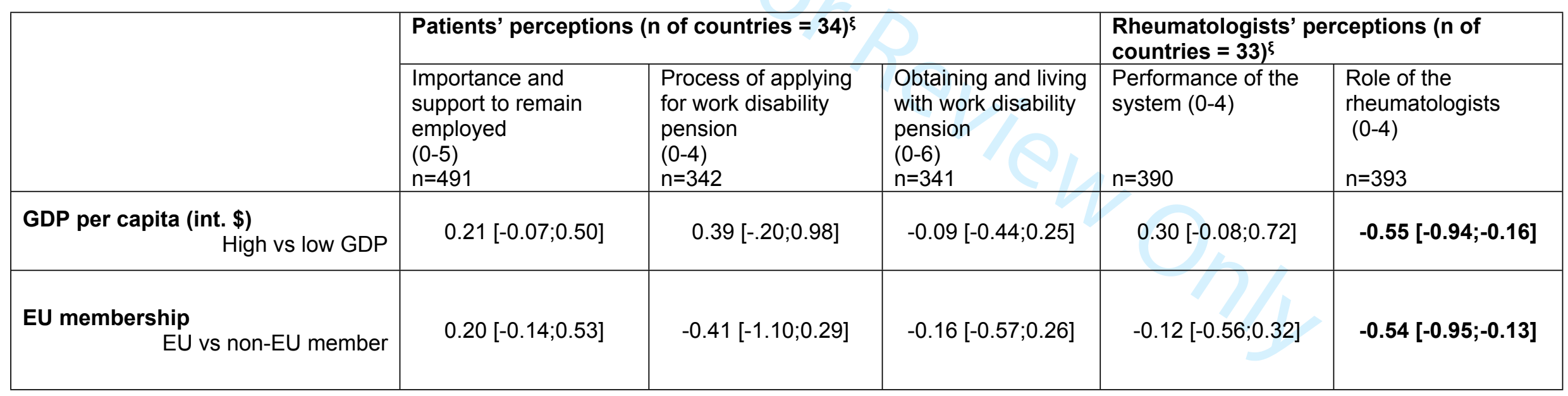




\section{Type of system}

(1)

\begin{tabular}{c|c} 
& \\
Reference & Reference \\
$-1.41[-2.73 ;-0.09]$ & $-0.47[-1.30 ; 0.36]$ \\
$-0.74[-1.84 ; 0.40]$ & $-0.04[-0.73 ; 0.65]$ \\
$-1.29[-2.33 ;-0.25]$ & $-0.20[-0.86 ; 0.46]$ \\
$-1.02[-1.91 ;-0.13]$ & $-0.04[-0.62 ; 0.53]$
\end{tabular}

Reference

Coefficients are derived from separate multilevel multiple models (with individuals clustered in countries and each independent variable (i.e. GDP, EU

membership or type of system), adjusted for age, gender, education, disease duration and ever having had sick leave due to RA; analyses on the rheumatologist domains were adjusted for age, gender and work setting; GDP - Gross Domestic Product, EU - European Union

Anglo-saxon: UK, Ireland; Scandinavian: Denmark, Iceland, Sweden, Norway, Finland; Bismarkian: Austria, Belgium, Germany, France, Israel, Netherlands, Switzerland; Mediterranean: Cyprus, Greece, Italy, Portugal, Spain, Turkey; Post-communist: Albania, Bulgaria, Czech Republic, Croatia, Estonia, Georgia, Hungary, Latvia, Lithuania, Poland, Romania, Russian Federation, Serbia, Tajikistan, Slovak Republic, Slovenia

sthe higher score the more positive are the perceptions

Statistically significant $(p<0.05)$ regression estimates are in bold 


\section{Discussion}

To our knowledge, this is the first study to provide an extended overview of systems to support work or work disability in RA. With regard to the system rules and regulations, i.e. availability and affordability of work disability arrangements, a large heterogeneity across countries was observed for most regulations including income compensation. While research and judgement on which system is preferable is complex and beyond the aim of this study, it is striking that a person with RA, upon becoming disabled, will face very different perspectives on future work participation, depending on his/her country of residence. Only in a minority of countries work re-integration plans were obligatory before starting a procedure towards WD.

Despite important variation, we could not detect patterns explaining differences in the formal rules and regulations of the SS systems, with only few exceptions. We could not find support to our first hypothesis that lower GDP countries would have stricter rules around work disability or lower relative income substation when WD is granted, nor did we find any common features of systems in lower GDP countries or EU vs non-EU member states. Moreover, the rules of the SS system did not seem to have a large role in explaining differences in WD in RA. Earlier research - mainly in general population and above 50 years of age in a number of European countries and dating back to 2007 - suggested that the national SS system and, in particular, generosity of benefits, explains a large proportion of between country variations in disability rates.[25] We could not reproduce these results in our study among patients with RA. It is worth noting that data on work disability rates in RA was available for 21 countries only and collected almost a decade earlier, and was based on a sample of patients rather than national statistics.[16]

In contrast to findings on formal regulations (availability and affordability), patients' and rheumatologists' perceptions of systems to support persons with RA encountering work restrictions (acceptability), showed an apparent variation according to the type of the social security system: the Scandinavian and Bismarkian employment support and social security system consistently appeared to most adequately meet the expectations of patients and rheumatologists regarding remaining at work and application for a work disability pension. At the same time, little differences were related to country's wealth or EU-membership, and only a weak signal suggested that rheumatologists in lower 
income countries are more confident in their role to support patient in work disability issues. The latter may indicate that rheumatologists in low income countries interact more intensively with patients on these issues and accept work disability questions as part of their responsibility. Alternatively, other cultural and system factors could be considered, e.g. patients referring to other professionals within the system (such as primary care or state agencies) for advice and support regarding work disability decisions.[26] Overall, a lack of standardisation in the decision-making process on work disability was reported by nearly three quarters of the rheumatologists, and reinforces earlier calls for efforts for standardisation and homogenisation.[27] Therefore, our third hypothesis that levels of satisfaction with the system is higher in EU member states and higher GDP countries was not supported by the available data, while initial insight was generated with respect to the social security system type where no hypotheses were formed a priori.

This study has some notable limitations. First, the complexity of access to social security related to work and disability is hard to capture with a questionnaire that unavoidably simplifies reality. In absence of a validated tool to measure formal social security regulations and perceptions around the system performance, we used selfdeveloped questionnaires. These happened in intensive collaboration with several international experts on work participation studies. We did not follow a formal translation procedure and the decision and responsibility to translate was left with the PI, however, the translations were double-checked by patient partners. While government authorities would be the most knowledgeable parties to enquire about rules and regulations, the feasibility issues around establishing a direct contact with agencies from $>30$ European countries, in different languages, and where they likely have little or no interest to contribute to a research project, led us to choose for the well-established network of rheumatologists active in research was approached instead. To improve data quality and accuracy, Pls were encouraged to seek help from other experts in their country; the summary was sent to available patient partners for a face-validity check of data in their country, and the results showed a good agreement. It is emphasized in the discussion that this study has an explorative nature and limited conclusions should be drawn.

We have to acknowledge that application of the formal rules and regulations can differ substantially from the formal rules, and hence patients in countries with similar rules 
could potentially have different experiences when rules are applied. For example, partial work disability (which formally implies that a patient can work part of the time) may be a barrier to any employment in some countries and thus perceived differently compared to countries where patients deemed partially disabled are able to use their right for parttime work. We have attempted to get the initial insight into this through the surveys among patients and rheumatologists. Furthermore, it was challenging to select the best country-level characteristics that should be related to indicators of access. On this line, the social security system taxonomy used[23] is most likely an oversimplification of the complex systems that are constantly changing and developing, but to our knowledge, no alternative taxonomy exists. Recourse to (official) disability is a complex construct in which social security has a limited role. Alternative approaches to gain insight into international variation should be considered to study the performance and impact or social security systems. One of the potentially promising method could consist of series of clinical vignettes. By considering the rules and regulations that are to be applied to a hypothetical patient with given characteristics in terms of disease, work situation and disability, as well as attitudes and values about role of work in life and society, countries could be compared and further classified. Despite limitations, we hereby present the first attempt to understand whether patterns in regulations can be found to help to understand differences in employment, sick leave and work disability. We found differences in regulations and income substitution that are challenging our perceptions of equity and call for further research to justify them or for efforts to define the acceptable standards. Cost-effectiveness models in RA often count with an improvement of work disability in parallel with the improvement of the health status of the patient (e.g. HAQ based Markov models). Our study suggests that large variations exist between countries regarding regulations of short term absence or work disability that can affect their chances to return to work, a point to consider in economic evaluations. Although we did not find clear relations between regulations and work participation rates, we should keep in mind the previous research that suggested that patients continue to work at different health status across countries.[16] As system type appears to have a rather limited impact on regulations and perceptions on them, this might indicate that interventions to support work retention in patients with RA could in principle be considered irrespective of social security system. Moreover, the ageing of 
the population worldwide urges policy-makers to increase the age of retirement, meaning that people with RA will also be expected to work longer in the years to come.

In conclusion, we observed large heterogeneity in rules and regulations of social security systems across Europe in relation to work disability of patients with RA, and these cannot be explained by existing welfare regimes, EU membership or country's wealth. These differences call for a platform to consider harmonization of policies for patients with RA who experience restrictions in work participation. While remarkably little differences of patients' and rheumatologists' perceptions are related to country's wealth and membership in EU, Scandinavian employment support and social security system appears to most adequately meet the expectations of patients and rheumatologists regarding endurable work participation and access to work disability pension.

What is already known about this subject?

- RA has a high impact on functional ability and work participation.

What does this study add?

- Large variation in social security regulations for sick leave and work disability for patients with RA was observed across countries.

- This heterogeneity cannot be explained by existing welfare regimes, EU membership or country's wealth.

How might this impact on clinical practice?

- Heterogeneity between countries regarding regulations for sick leave and work disability can affect patients' chances to return to work.

- These differences call for a platform to consider harmonization of policies for patients with RA who experience restrictions in work participation. 


\section{Competing interests}

Authors declare no conflict of interest.

\section{Contributorship}

$\mathrm{PP}, \mathrm{SR}$ and $\mathrm{AB}$ conceived the study idea, FG, MP, FS, TS, MdW, AW, AZ contributed to the protocol and conceptualisation, $A K$ advised on statistical analyses, $D A, F B, I B, S B, K C, P C, R C$, ED, ND, AF, OFG, GG, NG, PG, MH, IJ, JV, XJ, MKov, MKull, LCM, MM, SMP, IN, ON, IFP, KP, BR, HR, FS, GS, IS, NS, PS, RS, SSok, SShuk, AT, MT, TU, SV collected data. All authors read the manuscript draft and approved the final submission.

\section{Acknowledgements}

We would like to acknowelde our patient research partners for their invaluable contribution at different stages of this project: Eva Decantere (Belgium), Boryana Boteva (Bulgaria), Kouloumas Marios (Cyprus), Alena Slamova (Czech Republic), Lena Anderson (Denmark), Tiina Jasinski (Estonia), Sonia Trope (France), Dieter Wiek (Germany), Mary Vella (Malta), Margôt Bakkers (the Netherlands), Dragan Chichikj (Macedonia), Gerd Jenny Aanerud (Norway), Jolante Grygielska (Poland), Elsa Mateus (Portugal), Zabalan Codruta (Romania), Polina Pchelnikova (Russia), Petra Bednarova (Slovakia), Aladar Belec (Slovenia), Laly Alcaide (Spain), Ayhan Dinc (Turkey), Pamela Richards (UK), National Rheumatoid Arthritis Society (NRAS, UK).

\section{Funding info}

This study was partially funded by Abbvie in the context of Fit for Work initiative. SV was supported by Versus Arthritis (grant numbers 20385) and NIHR Manchester Biomedical Research Centre.

\section{Patient and Public Involvement}

Patient partners have been involved in design of the study, as well in developing and testing survey instruments in different countries.

\section{Ethical approval information}

The project has been approved by Maastricht Ethical Committee. Principal Investigators in each country were responsible for local ethical approvals, where necessary.

\section{Data sharing statement}


Most of the data is provided in the online appendices. Further details can be provided upon request.

Figures

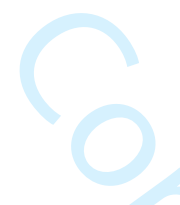

Figure 1. Study design.

\section{References}

1. Innala L, Berglin E, Moller B, et al. Age at onset determines severity and choice of treatment in early rheumatoid arthritis: a prospective study. Arthritis Res Ther 2014;16:R94

2. Cross $M$, Smith $E$, Hoy $D$, et al. The global burden of rheumatoid arthritis: estimates from the global burden of disease 2010 study. Ann Rheum Dis 2014;73:1316-22

3. Eriksson JK, Neovius M, Ernestam S, et al. Incidence of rheumatoid arthritis in Sweden: a nationwide population-based assessment of incidence, its determinants, and treatment penetration. Arthritis Care Res (Hoboken) 2013;65:870-8

4. Matcham F, Scott IC, Rayner L, et al. The impact of rheumatoid arthritis on quality-of-life assessed using the SF-36: a systematic review and meta-analysis. Semin Arthritis Rheum 2014;44:123-30

5. Lenssinck ML, Burdorf $A$, Boonen A, et al. Consequences of inflammatory arthritis for workplace productivity loss and sick leave: a systematic review. Ann Rheum Dis 2013;72:493-505

6. Backman CL. Employment and work disability in rheumatoid arthritis. Curr Opin Rheumatol 2004;16:148-52

7. Sokka T. Work disability in early rheumatoid arthritis. Clin Exp Rheumatol 2003;21:S71-4

8. Tiippana-Kinnunen T, Paimela L, Peltomaa R, et al. Work disability in Finnish patients with rheumatoid arthritis: a 15-year follow-up. Clin Exp Rheumatol 2014;32:88-94

9. Scott DL, Smith C, and Kingsley G. What are the consequences of early rheumatoid arthritis for the individual? Best Pract Res Clin Rheumatol 2005;19:117-36

10. Wolfe F, Michaud K, Choi HK, et al. Household income and earnings losses among 6,396 persons with rheumatoid arthritis. J Rheumatol 2005;32:1875-83

11. Angelini V, Cavapozzi D, and Paccagnella O, Dynamics of work disability reporting in Europe, in 'Marco Fanno' Working Paper (104). 2009: University of Padua.

12. OECD, Sickness, Disability and Work: Breaking the Barriers A Synthesis of Findings across OECD Countries. 2010.

13. Borsch-Supan A, Brugiavini A, and Croda E. The Role of Institutions and Health in European Patterns of Work and Retirement. J Eur Soc Policy 2009;19:341-358

14. Chung $C P$, Sokka T, Arbogast PG, et al. Work disability in early rheumatoid arthritis: higher rates but better clinical status in Finland compared with the US. Ann Rheum Dis 2006;65:1653-7

15. van der Zee-Neuen A, Putrik P, Ramiro S, et al. Large country differences in work outcomes in patients with RA - an analysis in the multinational study COMORA. Arthritis Res Ther 2017;19:216 
16. Sokka $\mathrm{T}$, Kautiainen $\mathrm{H}$, Pincus $\mathrm{T}$, et al. Work disability remains a major problem in rheumatoid arthritis in the 2000s: data from 32 countries in the QUEST-RA study. Arthritis Res Ther 2010;12:R42

17. European Commission, Reducing health inequalities in the European Union, in Luxembourg: Publications Office of the European Union. 2010.

18. de Croon EM, Sluiter JK, Nijssen TF, et al. Work ability of Dutch employees with rheumatoid arthritis. Scand J Rheumatol 2005;34:277-83

19. Odegard S, Finset A, Kvien TK, et al. Work disability in rheumatoid arthritis is predicted by physical and psychological health status: a 7-year study from the Oslo RA register. Scand J Rheumatol 2005;34:441-7

20. Mclntyre $D$, Thiede $M$, and Birch S. Access as a policy-relevant concept in low- and middleincome countries. Health Econ Policy Law 2009;4:179-93

21. Putrik $P$, Ramiro $S$, Kvien TK, et al. Inequities in access to biologic and synthetic DMARDs across 46 European countries. Annals of the rheumatic diseases 2014;73:198-206

22. IMF. 2011; World Economic Outlook Database (http://www.imf.org/external/pubs/ft/weo/2011/02/weodata/index.aspx). June, 2014.

23. Alvarez-Galvez J, Rodero-Cosano, M.L., García-Alonso, C. et al. Changes in socioeconomic determinants of health: comparing the effect of social and economic indicators through European welfare state regimes. J Public Health 2014;22:305

24. Sokka T, Kautiainen H, Toloza S, et al. QUEST-RA: quantitative clinical assessment of patients with rheumatoid arthritis seen in standard rheumatology care in 15 countries. Ann Rheum Dis 2007;66:1491-6

25. Börsch-Supan A, Work disability, health and incentive effects. MEA Discussion Paper (07135). 2007: University of Mannheim.

26. Ledingham JM, Snowden N, Rivett A, et al. Patient- and clinician-reported outcomes for patients with new presentation of inflammatory arthritis: observations from the National Clinical Audit for Rheumatoid and Early Inflammatory Arthritis. Rheumatology (Oxford) 2017;56:231-238

27. Barth J, de Boer WE, Busse JW, et al. Inter-rater agreement in evaluation of disability: systematic review of reproducibility studies. BMJ 2017;356:j14 
Title:

\title{
Patients with rheumatoid arthritis facing sick leave or work disability meet varying regulations: a study among rheumatologists and patients from 44 European countries
}

\begin{abstract}
Authors:
Polina Putrik ${ }^{* 1}$, Sofia Ramiro², Francis Guillemin ${ }^{3}$, Marta Pentek ${ }^{4}$, Francisca Sivera ${ }^{5}$, Tuulikki Sokka ${ }^{6}$,Maarten de Wit ${ }^{7}$, Anthony Woolf ${ }^{8}$, Angela Zink ${ }^{9}$, Daina Andersone ${ }^{10}$, Florian Berghea ${ }^{11}$, Irena Butrimiene ${ }^{12}$, Sandra Brouwer ${ }^{13}$, Karen Cassar ${ }^{14}$, Paraskevi Charalambous $^{15}$, Roberto Caporali ${ }^{16}$, Elena Deseatnicova ${ }^{17}$, Nemanja Damjanov ${ }^{18}$, Axel Finckh ${ }^{19}$, Oliver FitzGerald ${ }^{20}$, Gerður Gröndal ${ }^{21}$, Nino Gobejishjvili22, Piotr Gluszko ${ }^{23}$, Marco Hirsch ${ }^{24}$, Igor Jovanovic ${ }^{25}$, Jiri Vencovsky ${ }^{26}$, Xavier Janssens ${ }^{27}$, Andras Keszei ${ }^{28}$, Maria Kovarova ${ }^{29}$, Mart Kull ${ }^{30}$, Luis Cunha-Miranda ${ }^{31}$, Miroslav Mayer ${ }^{32}$, Snezana Misevska-Percinkova ${ }^{33}$, Inanc Nevsun ${ }^{34}$, Oleg Nadashkevich ${ }^{35}$, Ingemar F. Petersson ${ }^{36}$, Kari Poulakka ${ }^{37}$, B. Rojkovich ${ }^{38}$, Helga Radner ${ }^{39}$, Fruzsina Szabados ${ }^{40}$, Gleb Slobodin 41 , Ivan Shirinsky42, Nikolay Soroka ${ }^{43}$, Prodromos Sidiropoulos ${ }^{44}$, Russka Shumnalieva ${ }^{45}$, Sekib Sokolovic ${ }^{46}$, Surayo Shukurova ${ }^{47}$, Argiend Tafaj ${ }^{48}$, Matija Tomsic ${ }^{49}$, Till Uhlig ${ }^{50}$, Susanne Verstappen ${ }^{51}$, Annelies Boonen ${ }^{1}$
\end{abstract}

*corresponding author: polina.putrik@gmail.com

\footnotetext{
${ }^{1}$ Rheumatology, MUMC, Maastricht, the Netherlands

${ }^{2}$ Rheumatology, Leiden University Medical Centre, Leiden, and Zuyderland Medical Center, Heerlen, the Netherlands

${ }^{3}$ University of Lorraine, APEMAC, Nancy, France

${ }^{4}$ Corvinus University, Budapest, Hungary

${ }^{5}$ Hospital General Universitario de Elda, Alicante, Spain

6 Jyväskylä Central Hospital, Jyväskylä, Finland

${ }^{7}$ Amsterdam University Medical Centre, Dept. Medical Humanities, Amsterdam Public Health (APH), Amsterdam, Netherlands

${ }^{8}$ Royal Cornwall Hospital, Truro, United Kingdom

${ }^{9}$ German Rheumatism Research Centre and Rheumatology, Charité University Medicine, Berlin, Germany

10 Rheumatology, Riga, Latvia

${ }^{11}$ Carol Davila University of Medicine, Bucharest, Romania

${ }^{12}$ Center of Rheumatology, Institute of Clinical Medicine, Medical Faculty, Vilnius University, Vilnius, Lithuania

${ }^{13}$ Department of Community and Occupational Medicine, University of Groningen, University Medical

Center Groningen, The Netherlands

${ }^{14}$ Faculty of Medicine and Surgery, University of Malta, Malta

${ }^{15}$ Cyrus Society for Rheumatology, Cyprus

${ }^{16}$ Division of Rheumatology, Universiry of Pavia and IRCCS S. Matteo Foundation, Pavia, Italy

17 Department of Rheumatology and Nephrology, State Medical and Pharmaceutical Universtiy

Nicolae Testemitanu, Republic of Moldova

${ }_{18}$ University of Belgrade School of Medicine, Institute of Rheumatology, Belgrade, Serbia

${ }^{19}$ Division of Rheumatology, University Hospital of Geneva (HUG), Switzerland

${ }^{20}$ Dept Rheumatology, St. Vincent's University Hospital and Conway Institute for Biomolecular

Research, University College Dublin. Ireland

${ }^{21}$ Centre for Rheumatology Research, University Hospital, Iceland
} 
22 Health Center of Georgia, Tbilisi, Georgia

${ }^{23}$ Department of Rheumatology, National Institute of Geriatrics, Rheumatology and

Rehabilitation, Warszawa, Poland

${ }^{24}$ Luxembourg, Luxembourg

25 Podgorica, Montenegro

${ }^{26}$ Institute of Rheumatology, Prague, Czech Republic

27 Department of Rheumatology, Ghent University Hospital, Ghent, Belgium

${ }^{28}$ Medical Informatics, Uniklinik RWTH Aachen University, Aachen, Germany

29 Slovakia, St.CM University, Trnava, Slovakia

30 Tartu University, Department of Rheumatology and Internal Medicine and Viljandi Hospital, Tartu,

Estonia

31 Instituto Português de Reumatologia, Lisbon, Portugal

32 Division of Clinical Immunology and Rheumatology, Department of Internal Medicine, University Hospital Centre Zagreb and University of Zagreb, School of Medicine, Zagreb, Croatia

33 University Rheumatology Clinic, Medical Faculty, Former Yugoslav Republic of Macedonia

(FYROM)

${ }^{34}$ Division of Rheumatology, School of Medicine, Marmara University, Istanbul, Turkey

${ }^{35}$ Lviv National Medical University, Lviv, Ukraine

${ }^{36}$ Skåne University Hospital, Lund, Sweden and Clinical Sciences Lund, Lund University, Lund

Sweden

${ }^{37}$ South Karelia Central Hospital, Finland

${ }^{38}$ Polyclinic of the Hospitaller Brothers of St. John of God, Budapest, Hungary

${ }^{39}$ Department of Internal Medicine III, Division of Rheumatology, Medical University Vienna, Vienna,

Austria

${ }^{40}$ Department of Rheumatology, North Denmark Regional Hospital

${ }^{41}$ Rheumatology Unit, Bnai Zion Medical Center, Haifa, Israel

${ }^{42}$ Laboratory of clinical immunopharmacology, Clinical immunology institute, Novosibirsk, Russia

${ }^{43}$ Rheumatology, Minsk, Belarus

${ }^{44}$ Greece, Medical School, University of Crete, Greece

${ }^{45}$ Clinic of Rheumatology, University Hospital "St. Ivan Rilski", Department of Internal

Medicine, Medical University -Sofia, Bulgaria

${ }^{46}$ Sarajevo, Bosnia\&Herzegovina

${ }^{47}$ Medical Science Academy, Tajikistan, Dushanbe

48 Medical University Of Tirana, Rheumatology clinic, "Nene Tereza" Hospital, Tirane, Albania.

${ }^{49}$ Department of Rheumatology, University Medical Centre, Ljubljana, Slovenia

50 Diakonhjemmet Hospital, Oslo, Norway

${ }^{51}$ Arthritis Research UK Centre for Epidemiology, Division of Musculoskeletal \& Dermatological Sciences, School of Biological Sciences, Faculty of Biology, Medicine and Health, The University of Manchester, Manchester Academic Health Science Centre, Manchester, United Kingdom; NIHR Manchester Biomedical Research Centre, Central Manchester University Hospitals NHS Foundation Trust, Manchester Academic Health Science Centre, United Kingdom; Arthritis Research UK-MRC Centre for Musculoskeletal Health and Work, University of Southampton, United kingdom.

Word count: $3,249-\underline{690}$

\section{Working group:}

Ermir Tafaj (Albania), Edi Rembeci (Albania), Saskia Decuman (Belgium), Anastasia Tushina (Belarus), Iva Miteva (Bulgaria), Šárka Forejtová (Czech Republic), Esben Toftegaard Knudsen (Denmark), Maxime Wach (France), Matthieu Mangin (France), Márta Fekete (Hungary), Antonella Celano (Italy), Grainne O'Leary (Ireland), Giedre Dereseviciene (Lithuania), Mona Thorekildsen (Norway), Leszek Roszkowski (Poland), Claudia Handra (Romania), Inessa Samko (Russia), Jenny Hubertsson (Sweden), Tapparel Ludovic (Switzerland), Zumrad Hamroeva(Tajikistan), Ilker Yagcı (Turkey), Ana Zekovic (Serbia), James Anderson (UK)

\section{Acknowledgements to patient partners:}




\begin{abstract}
Eva Decantere (Belgium), Boryana Boteva (Bulgaria), Kouloumas Marios (Cypus), Alena Stamova (Gzech Republic), Lena Anderson (Denmark), Tiina Jasinski (Estonia), Sonia Trope (France), Dieter Wiek (Germany), Mary Vella (Malta), Margôt Bakkers (the Netherlands), Dragan Chichikj (Macedonia), Gerd Jenny Aanerud (Noway), Jolante Grygielska (Poland), Elsa Mateus (Portugal), Zabalan Codruta (Romania), Polina Pchelnikova (Russia), Petra Bednarova (Slovakia), Aladar Belec (Slovenia), Laly Alcaide (Spain), Ayhan Dinc (Turkey), Pamela Richards (UK), National Rheumatoid Arthritis-Society (NRAS, UK).
\end{abstract}

\title{
Abstract \\ Objectives
}

To describe and explore differences in formal regulations around sick leave and work disability (WD) for patients with rheumatoid arthritis (RA), as well as perceptions by rheumatologists and patients on the system's performance, across European countries.

\section{Methods}

We conducted three cross-sectional surveys in 50 European countries: one on work (re-)integration and social security (SS) system arrangements in case of sick leave and long-term WD due to RA (one rheumatologist per country), and two among approximately 15 rheumatologists and 15 patients per country on perceptions regarding SS arrangements on work participation. Differences in regulations and perceptions were compared across categories defined by Gross Domestic Product (GDP), type of social welfare regime, European Union (EU) membership and country RA WD rates.

\section{Results}

Forty-four (88\%) countries provided data on regulations, $33(75 \%)$ on perceptions of rheumatologists ( $n=539)$, and $34(77 \%)$ on perceptions of patients $(n=719)$. While large variation was observed across all regulations across countries, no relationship was found between most of regulations or income compensation and GDP, type of SS system, or rates of WD. Regarding perceptions, rheumatologists in high GDP and EUmember countries felt less confident in their role in the decision process towards WD ( $\beta=-0.5[95 \% \mathrm{CI}[-0.9 ;-0.2]$ and $\beta=-0.5[-1.0 ;-0.1]$, respectively). The Scandinavian and Bismarkian system scored best on patients' and rheumatologists' perceptions of regulations and system performance.

\section{Conclusions}

There is large heterogeneity in rules and regulations of SS systems across Europe in relation to WD of patients with RA, and it cannot be explained by existing welfare regimes, EU membership or country's wealth. 


\section{Introduction}

Rheumatoid arthritis (RA) is a chronic inflammatory joint disease that often starts during working life of patients.[1-3] RA has a high impact on functioning and ability to participate in social roles.[4, 5] Despite substantial improvements in treatment options in the last decades, still $20 \%$ of the patients are unable to continue to work in the first 3 years of disease and over $30 \%$ of patients become work disabled after 10 years.[69]

For patients, work disability (WD) implies exclusion from an essential role in society, but also loss of income and reduced economic self-sufficiency.[5, 10] To prevent individuals from poverty in case of WD, substitution of income has been introduced in some European countries in the $19^{\text {th }}$ century. By now, most countries have some form of social security (SS) system in place to regulate income substitution. However, these systems are not uniform, as they have been shaped by national political and social developments throughout the $20^{\text {th }}$ century. Policies targeted at income substitution balance between provision of a fair income, on one hand, and control of expenditures by restricting social security benefits only to individuals with specific levels of work restrictions, on the other hand.[11] More recently, the increasing economic burden of WD, but also the insight into social and health benefits of work participation stimulated in many countries stricter gate-keeping on the one hand, and stronger employment support to enhance endurable work participation of persons with chronic disease on the other hand.[12]

SS arrangements have been suggested to result in differences in overall patterns of employment, WD, and retirement.[13, 14] A recent worldwide multinational study (COMORA) has shown that lower economic wealth and human development of countries is associated with higher rates of unemployment and higher absenteeism.[15] Earlier, QUEST RA study has observed that in low Gross Domestic Product (GDP) countries, people remain working with higher levels of disability and disease activity compared to high GDP countries.[16] The regulations around the sick leave and work disability may be, at least partially, responsible for these differences, however, they have not been studied.

Policies with regard to work participation, however, go far beyond income substitution only. Criteria for access to WD benefits as well as levels of income substitution likely 
depend, among others, on economic, political and cultural factors. While the European Union (EU) and World Health Organization (WHO) accept the historical differences in the way health and social systems are organised and function in their member states, there is a universal agreement that differences should not result in inequalities in health and quality of life of people across nations.[17] It is unclear whether EU member states achieved any degree of homogeneity in the key regulations around social policies with regards to work disability. In addition to system level factors, there is evidence that personal contextual and disease related factors $[6,18,19]$, influence the decision of an individual to take sick leave or apply for long term WD.

The aim of this study was to describe and explore differences in formal regulations around sick leave and WD for patients with RA, as well as perceptions by rheumatologists and patients on the system's performance, across European countries. We hypothesised that 1) lower GDP countries have stricter rules with regards to obtaining work disability and lower income substitution once work disability is granted 2) EU countries have more homogeneous regulations compared to non-EU countries 3) patients and rheumatologists in high GDP and EU member states are more satisfied with the performance of the social security system.

\section{Methods}

\section{Design and framework}

We conducted a cross-sectional observational study consisting of three surveys in 50 of the 53 countries of the European WHO Region (in three countries, no contact person could be found), in 2014-2016 (Figure 1). The questionnaires were designed following the framework of access (originally applied in healthcare $[20,21]$ ), with three dimensions : i) availability of re-integration plans or other systems/policies to facilitate work and/or prevent WD, as well as the eligibility criteria that a patient should meet to receive SS benefits; ii) affordability, i.e. the level of income substitution granted in case of sick leave and permanent disability; iii) acceptability, i.e. professional and individual perceptions of rheumatologists and patients, respectively, around the system performance on these issues. The study has been approved by Maastricht Ethical Committee. 


\section{Participants}

For each of the 50 European countries, one rheumatologist was invited as principal investigator $\mathrm{PI}$ ) to complete the questionnaire on SS arrangements in his/her country in case of sick leave and long-term WD due to RA (survey 1; availability and affordability). Additionally, each PI was asked to invite at least 15 rheumatologists (survey 2; acceptability) and at least 15 patients (survey 3; acceptability) to complete a questionnaire on professional and individual perceptions of the system. To recruit rheumatologists, Pls were instructed to ensure a diverse sample in terms of gender, years of professional experience, and clinical setting. Patients could also be recruited via patient organizations and aimed at representing the spectrum of patients with RA, assuring that at least a half of them had experience with (applying for a) WD pension.

\section{Questionnaires}

The questionnaire for Pls addressed national regulations (in 2014) on benefits separately for sick leave and WD (Online Supplementary Text S1a: availability and affordability), as well as calculations of the level of income for three-nine pre-specified scenarios (vignettes), one on sick leave and two for long-term WD across the three levels of income (Online Supplementary Text S1b). In countries where a patient research partner was available $(n=21)$, he/she was invited to comment on any inconsistencies in the answers on the main questionnaire about the formal regulations. In this case, answers were double-checked with the PI.

The questionnaire for rheumatologists (Online Supplementary Text S2; acceptability) contained questions about perceptions on appropriateness of the SS arrangements, practical aspects of the application process for benefits, and the role of rheumatologists in the process. The questionnaire for patients (Online Supplementary Text S3; acceptability) addressed perceptions about the importance and adequacy of the existing arrangements. Additional questions were included on age, gender, work environment (non-university hospital, university hospital, private practice, other) for rheumatologists; on age, gender, disease duration, work status (paid work, no paid work but not work disabled, partially or fully work disabled) and history of sick leave and WD. The PI decided on whether questionnaires could be applied in English, otherwise translated them, wherever possible with patient partners involved in checking the translation. 
Data on Gross Domestic Product (GDP) per capita (in international dollars, 2013) were extracted from the World Development Indicators report by the World Bank [22] and used as a continuous variable or dichotomized around the median (27,000 int.\$). The welfare regimes taxonomy included five groups, namely the Anglo-Saxon, Bismarckian, Mediterranean, Post-Communist, and Scandinavian type of system.[23] Rates of work disability among RA patients have been collected by the QUEST-RA study (2009) and were available for 21 countries from our sample.[16, 24]

\section{Part I: Formal regulations and rules}

- One investigator per country filled in the questionnaire

\section{Part II: survey among rheumatologists}

- >15 rheumatologists per country on perceptions of social security system

\section{Part III: survey among patients}

- > 15 patients per country on perceptions of social security system

\section{Figure 1, Study-design}

\section{Statistical analysis}

Arrangements to support work and social security regulations in case of sick leave or work disability due to RA (questionnaire 1)

Collected data on formal rules and policies were first presented through descriptive statistics. To investigate whether the regulations differed by the type of welfare regime[23], GDP, EU membership (EU-15, new EU member states and non-EU countries), or were associated with country-level work disability rates among RA patients[16], subgroup comparisons were performed using Pearson correlations, ttest/Mann-Whitney and chi-square/Fisher's test, as appropriate.

Patients' and rheumatologists' perceptions of the social security system (questionnaires 2 and 3)

Answers of rheumatologists on their perspective of SS arrangements were summarized and scored (the higher is the better) around the two domains: (1) 
'Performance of the system' (score 0-4), questions Q2, Q8, Q9, Q11; Online Supplementary Text S2) and (2) 'Role of the rheumatologists' (score 0-4), Q3 Q4 Q5 Q10). Additionally, a single item on the perceived standardisation in the decisionmaking process (Q7) was analysed separately. Input from patients was summarized following three domains: (1) 'Importance and support to remain employed' (score 0-5; Q10, Q11, Q12, Q13, Q14, Online Supplementary Text S3), (2) 'Process of applying for WD (score 0-4, Q20, Q21, Q22, Q23, Q25), and (3) 'Obtaining and living with work disability pension' (score 0-6, Q15, Q16, Q17, Q18) (complete questionnaires are provided in the Online Supplementary Texts S2 and S3). Each domain consisted of 4 to 6 questions (each on a 1 (totally agree) to 5 (totally disagree) Likert scale, dichotomized as 1 ('(totally) agree') and 0 ('not agree/not disagree', '(totally) disagree'). The dichotomized scores per question were summed into the five domain scores (two for rheumatologists and three for patients).

Rheumatologists' and patients' characteristics were compared across the type of SS system, EU membership, and GDP. Small numbers of surveyed patients and rheumatologists in each country hindered analyses of country level means, and thus were not related to national RA work disability rates. The domain 'Importance and support to remain employed' was assessed in patients currently or ever having worked. Analyses in domains 'process of applying for work disability and 'obtaining and living with work disability were limited to patients currently work disabled or ever considered applying for WD.

Finally, we conducted multilevel (with individuals clustered in countries) multiple regression analysis with each of the domains as an outcome and type of SS system, EU membership or GDP as the independent variable of interest. Models with patient perceptions as outcome were adjusted for age, gender, education, disease duration and ever having had sick leave due to RA. When rheumatologists' perceptions were the outcome, analyses were adjusted for age, gender and work setting.

\section{Results}

Forty-four (28 EU and 16 non-EU member states) countries (88\%) provided data on formal rules and regulations for sick leave and WD. Of these, 33 (75\%) countries collected data from rheumatologists $(n=539)$, and $34(77 \%)$ countries collected data from patients $(n=719)$ (missing countries were all non-EU members except Luxemburg). 
Arrangements to support work and social security regulations in case of sick leave of work disability due to RA

While nearly all countries had arrangements to support patients with restrictions to work, a large heterogeneity was observed in the type of arrangements (Table 1, Online Supplementary Tables S1-3). All except for 12 countries had facilities to support patients with RA in paid employment $(n=32,73 \%)$, but only in a quarter of countries $(n=11,25 \%)$ rehabilitation efforts were obligatory prior to the decision about long-term WD. Twenty-five $(57 \%)$ and $30(68 \%)$ countries had a requirement for employment history or social insurance contributions in order to be eligible for sick leave or longterm WD compensation, respectively. The maximum sick leave length before transition to long-term WD varied from 3 to 36 months (mean (SD) 13 (9)). In 8 (18\%) and 5 $(11 \%)$ of the countries, participation of a rheumatologist was mandatory in the process of application or decision-making process on long-term WD, respectively. In addition to a functional assessment (degree of (dis)ability), prior profession, $(n=25(57 \%)$ of countries), diagnosis $(n=32(73 \%))$, earning capacity $(n=12(27 \%))$, age $(n=20(45 \%))$, and gender $(n=5(11 \%)$ ) were reported to be accounted for when a decision was taken about (the degree of) WD. Prognosis, education and place of residence were mentioned as additional factors by few countries.

All countries except the former Yugoslav Republic of Macedonia, Bosnia-Herzegovina and Serbia reported to recognise partial WD, a status that partially substitutes income while the person can continue in (reduced) employment. Of the 26 countries providing data on income substitution, in 8 countries (31\%) income substitution (averaged over the first 6 months of disability) in case of sick leave was less than $70 \%$ of previous income. Income substitution averaged over the first 12 months of disability was less than $70 \%$ in $18(70 \%)$ and $15(58 \%)$ in case of moderate (partial) or severe (full) longterm disability, respectively (Table 2). While wealthier countries as expected provided higher benefits in absolute terms, when converted to percentage of the previously earned income, no relationship was found between income compensation and neither GDP, nor the type of SS system, or rates of work disability. In richer countries and in countries with the Bismarkian type of welfare regime, the WD pension burden was more likely to be shared between SS and a private insurance, while countries with lower GDP and other welfare regimes had social insurance as the main source of WD allowances (data not shown). In countries with lower GDP, a rheumatologist was more frequently necessarily involved in the application and decision making. Other aspects 
of the system revealed no statistically significant patterns with country-level characteristics or national WD rates.

Rheumatologists' and patients' and perceptions of social security system

In total, 539 rheumatologists (mean age (SD) 48 (10), 284 (53\%) female, 278 (51\%) working in university hospitals) from 33 countries filled in the questionnaires (Table Online Supplementary Table 3 S4). Scores on 'Role of the rheumatologists' (0-4) and 'Performance of the system' (0-4) ranged from 1.4 (SD 0.9) (Anglo-Saxon) to 2.4 (1.1) (Post-communist) and 0.8 (0.9) (Anglo-Saxon) to 2.3 (1.2) (Scandinavian), respectively. Perceived level of standardization around decision-taking revealed that only $26 \%$ ( $n=135)$ of rheumatologists consider decisions on WD to be objective. Of note, those who perceived standardization as poor (vs good) scored worse on both 'Role of the rheumatologists' (-0.4 points) and 'Performance of the system' (-2.6 points) domains (t-test $p$-value for both scores <0.05). Remarkably, mMultilevel analyses revealed that rheumatologists in high GDP (vs low GDP) and EU-member (vs non-EU member) countries felt less confident in having an active role in WD decisions $(\beta=-0.5[95 \% \mathrm{Cl}[-0.9 ;-0.2]$ and $\beta=-0.5[-1.0 ;-0.1]$, respectively). In addition, significant differences were observed across the system types with the Scandinavian type (Denmark, Iceland, Sweden, Norway, Finland) consistently scoring higher than the others on domains 'Importance and support to remain employed' and 'Process of applying for work disability pension' (Table 4 and Supplementary Table S5).

The patient sample consisted of 719 patients from 34 countries (mean age (SD) 53 (12), $76 \%$ female, $519(78 \%)$ ever worked). The highest (=most satisfied) patient scores on all three domains were consistently observed in countries with Scandinavian and Bismarkian type of security system (Table 3). In multilevel adjusted regression models, neither country wealth nor EU status were associated with patients' perceptions (Table 4 and Supplementary Table S5). The findings across the system type were notably consistent across patient and rheumatologist domains. 
Table 1. Summary of social security regulations to support stay at work and work disability arrangements for persons with RA

\begin{tabular}{|c|c|c|c|c|c|c|c|}
\hline \multicolumn{2}{|l|}{ Regulation } & $\mathbf{N}(\%)^{*}$ & $\begin{array}{l}\text { Anglo- } \\
\text { saxon }\end{array}$ & Scandinavian & Bismarkian & Mediterranean & Post-communist \\
\hline \multicolumn{8}{|l|}{ Support to stay at work } \\
\hline \multirow{2}{*}{$\begin{array}{l}\text { Facilities for persons with RA } \\
\text { that aimed at keeping the } \\
\text { patient in paid employment are } \\
\text { available }\end{array}$} & Yes & $32(73 \%)$ & IE, $\underline{\text { GB }}$ & $\frac{\mathrm{DK}}{\mathrm{SE}}, \underline{\mathrm{FI}}, \underline{\mathrm{IS}}, \underline{\mathrm{NO}}$ & $\begin{array}{l}\frac{\mathrm{AU}}{\mathrm{LU}}, \underline{\mathrm{BE}}, \underline{\mathrm{DE}}, \\
\mathrm{NL},\end{array}$ & CY, IT, PT & $\begin{array}{l}\text { AL, BY, BG, CR, CZ, LT } \\
\text { MK, MD, ME, PL, RO, } \\
\text { RS, SK, SL, TJ }\end{array}$ \\
\hline & No & $12(27 \%)$ & - & $F$ & FR & $\begin{array}{l}\text { GR, MT, ES, } \\
\text { TR }\end{array}$ & $\begin{array}{l}\mathrm{BA}^{\mathrm{P}} \mathrm{EE}^{\mathrm{P}} \mathrm{GE}^{\mathrm{P}} \mathrm{HU}^{\mathrm{P}} \mathrm{RU}^{\mathrm{P}} \\
\text { LV }^{\mathrm{P}} \mathrm{UA}^{\mathrm{P}}\end{array}$ \\
\hline \multicolumn{8}{|c|}{ Access to sick-leave or short term absence } \\
\hline \multirow{2}{*}{$\begin{array}{l}\text { Requirement for length of } \\
\text { previous employment AND/OR } \\
\text { certain income earned } \\
\text { AND/OR certain amount of } \\
\text { social insurance tax paid in } \\
\text { order to be eligible for sick } \\
\text { leave compensation }\end{array}$} & Yes & $25(57 \%)$ & $\underline{\text { IE, }}, \underline{\mathbf{G B}}$ & DK, NO, SE & $\mathrm{BE}, \underline{\mathrm{FR}}, \underline{\mathrm{IL}}$, & \begin{tabular}{|l}
$\mathrm{CY}, \mathrm{GR}, \underline{\mathrm{PT}}$, \\
$\mathrm{ES}$
\end{tabular} & $\begin{array}{l}\text { AL, BY, BA, BG, HR, } \\
\text { CZ, LT, MD, PL, RO, } \\
\text { SK, TJ } \\
\end{array}$ \\
\hline & No & $18(41 \%)$ & & FI, IS & $\frac{\mathrm{AU}}{\mathrm{NL}}, \underline{\mathrm{DE}}, \underline{\mathrm{LU}}$, & IT, MT, TR & $\begin{array}{l}\text { EE, GE, HU, LV, MK, } \\
\text { ME, RU, RS, SL, UA }\end{array}$ \\
\hline \multirow{2}{*}{$\begin{array}{l}\text { The rheumatologist is } \\
\text { authorized to certify short-term } \\
\text { absence from work due to RA }\end{array}$} & Yes & $33(75 \%)$ & $\underline{\text { IE }}$ & $\frac{\mathrm{DK}}{\mathrm{SE}}, \underline{\mathrm{FI}}, \underline{\mathrm{IS}}, \underline{\mathrm{NO}}$ & 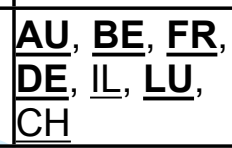 & \begin{tabular}{|l} 
CY, GR,,$\underline{\text { MT, }}$ \\
PT, ES, \\
TR
\end{tabular} & $\begin{array}{l}\text { AL, BY, BA, CZ, EE, } \\
\text { HU, LT, MK, ME, PL, } \\
\text { RO, RU, TJ, UA }\end{array}$ \\
\hline & No & $10(23 \%)$ & $\underline{\text { GB }}$ & & $\mathrm{NL}$ & IT & $\begin{array}{l}\text { BG, CR, GE, LV, MD, } \\
\text { RS, SK, SL }\end{array}$ \\
\hline \multirow[b]{2}{*}{$\begin{array}{l}\text { First 1-3 days of sick leave are } \\
\text { unpaid }\end{array}$} & Yes & $11(25 \%)$ & - & SE & FR, IL & $\frac{\mathrm{CY}}{\mathrm{TR}}, \underline{\mathrm{IT}}, \underline{\mathrm{PT}}, \underline{\mathrm{ES}}$, & CZ, EE, LV \\
\hline & No & $32(73 \%)$ & IE, $\underline{\text { GB }}$ & 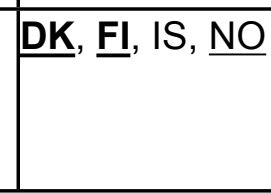 & 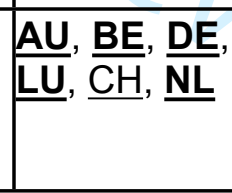 & GR, MT & $\begin{array}{l}\text { AL, BY, BA, BG, CR, } \\
\text { GE, HU, LT, MK, MD, } \\
\text { ME, PL, RO, RU, RS, } \\
\text { SK, SL, TJ, UA }\end{array}$ \\
\hline \multicolumn{8}{|c|}{ Decision about long-term work disability } \\
\hline $\begin{array}{l}\text { Maximum length of sick leave } \\
\text { before a WD can be granted }\end{array}$ & $\begin{array}{l}<12 \\
\text { months }\end{array}$ & $35(80 \%)$ & $\underline{\mathrm{GB}}$ & DK, IS, $\underline{\text { SE }}$ & $\frac{\mathrm{AU}}{\mathrm{IL}}, \underline{\mathrm{LR}}, \underline{\mathrm{DE}}$, & \begin{tabular}{|l} 
CY, GR, IT, \\
MT, PT, ES, \\
TR
\end{tabular} & $\begin{array}{l}\text { AL, BY, BG, CR, EE, } \\
\text { LV, LT, HU, MK, MD, } \\
\text { ME, PL, RO, RU, RS, } \\
\text { SL, TJ, UA }\end{array}$ \\
\hline
\end{tabular}




\begin{tabular}{|c|c|c|c|c|c|c|c|}
\hline \multicolumn{8}{|c|}{ Annals of the Rheumatic Diseases } \\
\hline & $\begin{array}{l}\geq 12 \\
\text { months }\end{array}$ & $8(18 \%)$ & IEE & FI, NO & $\mathrm{BE}, \underline{\mathrm{CH}}, \underline{\mathrm{NL}}$ & - & BA, $\underline{\mathbf{C Z}}, \mathbf{S K}$ \\
\hline \multirow{2}{*}{$\begin{array}{l}\text { Is it obligatory that a } \\
\text { rheumatologist is involved in } \\
\text { application process (i.e. } \\
\text { providing information about the } \\
\text { disease)? }\end{array}$} & Yes & $10(23 \%)$ & - & - & - & CY, GR & $\begin{array}{l}\text { AL, BY, BG, LT, } \\
\text { MK,SK,RU, TJ }\end{array}$ \\
\hline & No & $31(70 \%)$ & $\underline{\underline{\mathrm{IE}}}, \underline{\mathrm{GB}}$ & DK, FI, NO & $\begin{array}{l}\mathrm{AU}, \mathrm{BE}, \overline{\mathrm{FR}}, \\
\mathrm{IL}, \mathrm{LU}, \underline{\mathrm{CH}}, \\
\mathrm{NL}, \mathrm{DE}\end{array}$ & $\frac{\mathrm{IT}}{\mathrm{ES}}, \frac{\mathrm{MT}}{\mathrm{TR}}, \underline{\mathrm{PT}}$ & $\begin{array}{l}\text { BA, CR, CZ, EE, HU, LV } \\
\text {, MD, PL, RO, RS, } \underline{\text { SL, }} \\
\text { UA }\end{array}$ \\
\hline \multirow[b]{2}{*}{$\begin{array}{l}\text { Is the rheumatologist also in } \\
\text { an obligatory way involved in } \\
\text { the decision-taking about the } \\
\text { long-term work disability? (i.e. } \\
\text { is part of the commission or } \\
\text { expert committee) } \xi\end{array}$} & Yes & $5(11 \%)$ & - &  & - & CY & AL, BG, SK, TJ \\
\hline & No & $36(82 \%)$ & $\underline{\underline{\mathrm{IE}}}, \underline{\mathrm{GB}}$ & DK, FI, NO & $\begin{array}{l}\mathrm{AU}, \mathrm{BE}, \mathrm{FR}, \\
\mathrm{IL}, \underline{\mathrm{LU}}, \underline{\mathrm{CH}}, \\
\mathrm{NL}, \mathrm{DE}\end{array}$ & $\begin{array}{l}\text { GR, IT, }, \frac{\mathrm{MT}}{\mathrm{PT}}, \underline{\mathrm{ES}}, \mathrm{TR} \\
\text { PI }\end{array}$ & \begin{tabular}{|l|} 
BY, BA, CR, CZ, EE, \\
HU, LV, LT, MK, MD, \\
PL, RO, RU, RS, SL, UA
\end{tabular} \\
\hline \multirow{2}{*}{$\begin{array}{l}\text { Rehabilitation efforts are } \\
\text { obligatory before a patient } \\
\text { could be assessed for long- } \\
\text { term disability }\end{array}$} & Yes & $11(25 \%)$ & - & DK, FI, SE, & $\mathrm{AU}, \underline{\mathrm{CH}}, \underline{\mathrm{NL}}$ & - & LT, MD, ME, RO, SL \\
\hline & No & $32(73 \%)$ & $\underline{\text { IE, }} \underline{\text { GB }}$ & IS & $\begin{array}{l}\mathrm{BE}, \underline{\mathbf{F R}}, \underline{\mathrm{DE}}, \\
\mathrm{IL}, \underline{\mathrm{LU}}\end{array}$ & $\begin{array}{l}\text { CY, GR, IT, } \\
\frac{\mathrm{MT}}{\mathrm{TR}}, \underline{\mathrm{PT}}, \underline{\mathrm{ES}},\end{array}$ & \begin{tabular}{|l|} 
AL, BY, BA, BG, CR, \\
CZ, EE, HU, LV, MK, \\
PL, RU, RS, SK, TJ, UA
\end{tabular} \\
\hline
\end{tabular}

AL, Albania; AT, Austria; BA, Bosnia and Herzegovina; BE, Belgium; BG, Bulgaria; BY, Belarus; HR, Croatia, CH, Switzerland; CY, Cyprus; CZ, Czech Republic; DE, Germany; GE, Georgia; DK, Denmark; EE, Estonia; ES, Spain; FI, Finland; FR, France; GR, Greece; HU, Hungary; HR, Croatia; IE, Ireland; IS, Iceland; IL, Israel; IT, Italy; LT, Lithuania; LU, Luxemburg; LV, Latvia; MD, Moldova; ME, Montenegro; MK, Macedonia (the former Yugoslav Republic of Macedonia); MT, Malta; NL, The Netherlands; NO, Norway; PL, Poland; RO, Romania; RU, Russia; SE, Sweden; SK, Slovakia; SL, Slovenia; RS, Serbia; TR, Turkey; TJ, Tajikistan, UK, United Kingdom; UA, Ukraine *data provided by 44 countries, Georgia reported to not have any effective social security system in place; $\xi$ : data missing for IS, ME EU countries are in bold, High GDP (>27,000 int. dollars, based on median) countries are underscored 
Table 2. Income compensation in case of work disability for an employee with average income*

\begin{tabular}{|c|c|c|c|c|c|c|c|}
\hline \multirow{2}{*}{\begin{tabular}{|l|}
$\begin{array}{l}\text { Type of } \\
\text { disability }\end{array}$ \\
In case of sick \\
leave \\
\end{tabular}} & \multicolumn{2}{|c|}{ Income compensation } & \multirow{2}{*}{\begin{tabular}{|l|} 
Anglo-saxon \\
$\underline{\text { IE }}$ \\
\end{tabular}} & \multirow{2}{*}{\begin{tabular}{|l} 
Scandinavian \\
-
\end{tabular}} & \multirow{2}{*}{\begin{tabular}{|l|} 
Bismarkian \\
$\mathrm{BE}$
\end{tabular}} & \multirow{2}{*}{\begin{tabular}{|l|} 
Mediterranean \\
$\underline{\mathrm{CY}}, \underline{\mathrm{PT}}, \mathrm{TR}$
\end{tabular}} & \multirow{2}{*}{\begin{tabular}{|l|}
$\begin{array}{l}\text { Post- } \\
\text { communist }\end{array}$ \\
CZ, EE, SK \\
\end{tabular}} \\
\hline & $\begin{array}{l}\leqslant 70 \% \text { of earned } \\
\text { income }\end{array}$ & $8(31 \%)^{\star \star}$ & & & & & \\
\hline & $\begin{array}{l}>70 \% \text { of earned } \\
\text { income }\end{array}$ & $18(69 \%)$ & - & FI, NO & $\underline{L U}, \underline{N L}, \underline{S E}$ & FR, IT, ES & $\begin{array}{l}\text { BG, LT, LV, } \\
\text { MD, PL, RO, } \\
\text { RS, SL }\end{array}$ \\
\hline \multirow[t]{2}{*}{$\begin{array}{l}\text { In case of } \\
\text { moderate }(50 \%) \\
\text { work disability }\end{array}$} & $\begin{array}{l}\leq 70 \% \text { of earned } \\
\text { income }\end{array}$ & $18(70 \%)$ & $\underline{\text { IE }}$ & FI, NO & $\mathrm{BE}, \underline{\mathrm{SE}}, \underline{\mathrm{CH}}$ & CY, TR & $\begin{array}{l}\text { BY, BG, CZ, } \\
\text { EE, LV, LT, } \\
\text { MD, PL, RO, } \\
\text { RS, SK }\end{array}$ \\
\hline & $\begin{array}{l}>70 \% \text { of earned } \\
\text { income }\end{array}$ & $7(27 \%)$ & - & - & $\underline{L U}, \underline{\mathrm{NL}}$ & $\mathrm{FR}, \underline{\mathrm{IT}}, \underline{\mathrm{PI}}, \underline{\mathrm{ES}}$ & $A L$ \\
\hline \multirow[t]{2}{*}{$\begin{array}{l}\text { In case of } \\
\text { severe }(75 \%) \\
\text { work disability }\end{array}$} & $\begin{array}{l}\leq 70 \% \text { of earned } \\
\text { income }\end{array}$ & $15(58 \%)$ & & FI, NO & BE, LU, SE, $\underline{\text { CH }}$ & CY, & \begin{tabular}{|l} 
CZ, EE, LV, LT, \\
MD, PL, RO, \\
RS, SK \\
\end{tabular} \\
\hline & $\begin{array}{l}>70 \% \text { of earned } \\
\text { income }\end{array}$ & $10(38 \%)$ & $\underline{\text { IE }}$ & & $\mathrm{NL}$ & FR, IT, PI, ES, & $A L, B Y, B G$ \\
\hline
\end{tabular}

AL, Albania; BY, Belarus, BE; Belgium; BG; CY, Cyprus; CZ, Czech Republic; EE, Estonia; FI, Finland; FR, France; IE, Ireland; IT, Italy; LV, Latvia; LT, Lithuania;

LU, Luxemburg; MD, Moldova; NO, Norway; PL - Poland; PT, Portugal; RO, Romania; RS, Serbia; SK, Slovakia; SL, Slovenia; ES, Spain; CH, Switzerland; NL, the Netherlands; TR, Turkey

EU countries are in bold, High GDP (>27,000 int. dollars, based on median) countries are underscored ${ }^{*} \mathrm{SL}$ did not provide data for long term WD

*a person 50 y.o. recently diagnosed with RA, who is a citizen and has worked for 25 years full-time

${ }^{* *}$ data provided by 26 countries 
Table 3. Patients' and rheumatologists' characteristics per type of security system.

\begin{tabular}{|c|c|c|c|c|c|c|c|}
\hline & Total & Anglo-saxon & Scandinavian & Bismarkian & Mediterranean & Post-communist & p-value \\
\hline \multicolumn{8}{|l|}{ Rheumatologists characteristics (Questionnaire 2) } \\
\hline $\mathrm{N}$ of rheumatologists ( $\mathrm{N}$ of countries) & $539(33)$ & $22(2)$ & $58(5)$ & $88(6)$ & $87(6)$ & $284(15)$ & \\
\hline Age (years) & $47.5(10.2)$ & $45.1(8.1)$ & $51.8(10.5)$ & $51.1(9.5)$ & $47.4(9.5)$ & $45.5(10.3)$ & $\leq 0.001$ \\
\hline Gender, female & $284(53 \%)$ & $9(41 \%)$ & $25(43 \%)$ & $38(43 \%)$ & $45(52 \%)$ & $167(59 \%)$ & $\leq 0.01$ \\
\hline $\begin{array}{l}\text { Gurrent work environment } \\
\qquad \begin{array}{r}\text { Non-university hospital*t } \\
\text { University hospital } \\
\text { Private practice } \\
\text { Other }\end{array}\end{array}$ & $\begin{array}{l}142(26 \%) \\
278(51 \%) \\
52(10 \%) \\
22(4 \%)\end{array}$ & $\begin{array}{l}10(45 \%) \\
7(32 \%) \\
1(5 \%) \\
1(5 \%)\end{array}$ & $\begin{array}{l}12(21 \%) \\
39(67 \%) \\
0(0 \%) \\
0(0 \%)\end{array}$ & $\begin{array}{l}19(22 \%) \\
48(55 \%) \\
14(16 \%) \\
5(6 \%)\end{array}$ & $\begin{array}{l}23(26 \%) \\
50(57 \%) \\
8(9 \%) \\
4(5 \%)\end{array}$ & $\begin{array}{l}78(27 \%) \\
132(46 \%) \\
29(10 \%) \\
12(4 \%)\end{array}$ & $<0.001$ \\
\hline Years of work experience & $2.5(1.1)$ & $2.6(1.1)$ & $2.4(1.2)$ & $2.9(1.1)$ & $2.7(1.0)$ & $2.4(1.2)$ & $<0.001$ \\
\hline Average hours of patient care per week & $33.5(12.3)$ & $32.2(12.2)$ & $29.9(10.8)$ & $32.0(12.9)$ & $32.0(11.9)$ & $35.4(12.2)$ & $\leq 0.05$ \\
\hline Role of the rheumatologists $(0-4)$ & $2.1(1.1)$ & $1.4(0.9)$ & $1.3(0.9)$ & $2.1(1.3)$ & $1.9(1.0)$ & $2.4(1.1)$ & $<0.001$ \\
\hline Performance of the system (0-4) & $1.5(1.1)$ & $0.8(0.9)$ & $2.3(1.2)$ & $2.0(1.1)$ & $1.1(1.0)$ & $1.4(1.1)$ & $<0.001$ \\
\hline \multicolumn{8}{|l|}{ Patient characteristics (Questionnaire 3) } \\
\hline $\mathrm{N}$ of patients ( $\mathrm{N}$ of countries) & $719(34)$ & $47(2)$ & $60(5)$ & $137(7)$ & $119(6)$ & $356(16)$ & \\
\hline Age (years) & $53.1(12.2)$ & $48.6(9.9)$ & $57.9(11.0)$ & $54.7(11.9)$ & $52.2(12.1)$ & $52.7(12.4)$ & $\leq 0.001$ \\
\hline Gender, female & $541(76 \%)$ & $44(94 \%)$ & $48(80 \%)$ & $101(74 \%)$ & $90(76 \%)$ & $261(74 \%)$ & $\leq 0.05$ \\
\hline $\begin{array}{r}\text { Primary } \\
\text { Secondary } \\
\text { High }\end{array}$ & $\begin{array}{l}110(16 \%) \\
254(36 \%) \\
339(48 \%)\end{array}$ & $\begin{array}{l}0(0 \%) \\
14(30 \%) \\
32(70 \%)\end{array}$ & $\begin{array}{l}15(25 \%) \\
16(27 \%) \\
28(47 \%)\end{array}$ & $\begin{array}{l}16(12 \%) \\
31(24 \%) \\
82(64 \%)\end{array}$ & $\begin{array}{l}30(25 \%) \\
43(36 \%) \\
45(38 \%)\end{array}$ & $\begin{array}{l}49(14 \%) \\
150(43 \%) \\
151(43 \%)\end{array}$ & $<0.01$ \\
\hline $\begin{array}{r}\text { Paid work } \\
\text { No paid work* } \\
\text { Work disabled (WD) } \\
\text { Paid work + WD }\end{array}$ & $\begin{array}{l}317(45 \%) \\
180(26 \%) \\
172(24 \%) \\
34(5 \%)\end{array}$ & $\begin{array}{l}21(47 \%) \\
13(29 \%) \\
9(2 \%) \\
z(4 \%)\end{array}$ & $\begin{array}{l}23(38 \%) \\
12(20 \%) \\
16(27 \%) \\
9(15 \%)\end{array}$ & $\begin{array}{l}75(55 \%) \\
31(23 \%) \\
24(18 \%) \\
3(2 \%)\end{array}$ & $\begin{array}{l}57(49 \%) \\
36(31 \%) \\
22(19 \%) \\
1(1 \%)\end{array}$ & $\begin{array}{l}140(40 \%) \\
88(25 \%) \\
101(29 \%) \\
19(6 \%)\end{array}$ & $<0.01$ \\
\hline Symptom duration (years) & $15.0(11.1)$ & $13.3(9.6)$ & $17.7(11.2)$ & $14.6(11.8)$ & $15.8(10.4)$ & $14.8(11.1)$ & 0.22 \\
\hline Ever absent from work due to $R A$ & $472(69 \%)$ & $33(89 \%)$ & $42(71 \%)$ & $83(63 \%)$ & $74(64 \%)$ & $239(70 \%)$ & $<0,05$ \\
\hline $\begin{array}{l}\text { Importance and support to remain employed } \\
(0-5)\end{array}$ & $2.1(1.2)$ & $1.6(0.8)$ & $2.6(1.2)$ & $2.3(1.4)$ & $2.1(1.2)$ & $2.0(1.1)$ & $<0.001$ \\
\hline Process of applying for WD (0-4) & $2.2(1.8)$ & $1.3(1.8)$ & $3.2(3.0)$ & $2.4(1.9)$ & $2.0(1.6)$ & $2.3(1.7)$ & $<0.001$ \\
\hline Obtaining and living with WD pension (0-6) & $1.1(1.1)$ & $0.7(0.8)$ & $1.2(1.1)$ & $1.2(1.4)$ & $1.0(1.1)$ & $1.1(1.1)$ & 0.22 \\
\hline
\end{tabular}

Values reflect mean (SD) or $n(\%)$, as appropriate; WD - work disability * but not work disabled ** private or public Anglo-saxon: UK, Ireland; Scandinavian: Denmark,

Iceland, Sweden, Norway, Finland; Bismarkian: Austria, Belgium, Germany, France, Israel, Netherlands, Switzerland; Mediterranean: Cyprus, Greece, Italy, Portugal, Spain,

Turkey; Post-communist: Albania, Bulgaria, Czech Republic, Croatia, Estonia, Georgia, Hungary, Latvia, Lithuania, Poland, Romania, Russian Federation, Serbia, Tajikistan,

Slovak Republic, Slovenia 
Table 4. Patients' and rheumatologists' perceptions across several domains according to (1) GDP per capita PPP (2) EU membership status (3) Type of social security system. Models adjusted for socio-demographic confounders.

\begin{tabular}{|c|c|c|c|c|c|}
\hline & \multicolumn{3}{|c|}{ Patients' perceptions ( $n$ of countries $=34)^{\xi}$} & \multicolumn{2}{|c|}{$\begin{array}{l}\text { Rheumatologists' perceptions (n of } \\
\text { countries }=33)^{\xi}\end{array}$} \\
\hline & $\begin{array}{l}\text { Importance and } \\
\text { support to remain } \\
\text { employed } \\
(0-5) \\
n=491\end{array}$ & $\begin{array}{l}\text { Process of applying } \\
\text { for work disability } \\
\text { pension } \\
(0-4) \\
n=342\end{array}$ & $\begin{array}{l}\text { Obtaining and living } \\
\text { with work disability } \\
\text { pension } \\
(0-6) \\
n=341\end{array}$ & $\begin{array}{l}\text { Performance of the } \\
\text { system }(0-4) \\
n=390\end{array}$ & $\begin{array}{l}\text { Role of the } \\
\text { rheumatologists } \\
(0-4) \\
n=393\end{array}$ \\
\hline $\begin{array}{l}\text { GDP per capita (int. \$) } \\
\text { High vs low GDP }\end{array}$ & $0.21[-0.07 ; 0.50]$ & $0.39[-.20 ; 0.98]$ & $-0.09[-0.44 ; 0.25]$ & $0.30[-0.08 ; 0.72]$ & $-0.55[-0.94 ;-0.16]$ \\
\hline $\begin{array}{l}\text { EU membership } \\
\qquad E U \text { vs non-EU member }\end{array}$ & $0.20[-0.14 ; 0.53]$ & $-0.41[-1.10 ; 0.29]$ & $-0.16[-0.57 ; 0.26]$ & $-0.12[-0.56 ; 0.32]$ & $-0.54[-0.95 ;-0.13]$ \\
\hline $\begin{array}{r}\text { Type of system } \\
\text { Scandinavian } \\
\text { Anglo-Saxon/liberal } \\
\text { Bismarckian/conservative } \\
\text { Mediterranean/southern } \\
\text { Post-Communist/eastern }\end{array}$ & $\begin{array}{c}\text { Reference } \\
-1.02[-1.64 ;-0.39] \\
-0.40[-0.90 ; 0.10] \\
-0.56[-1.06 ;-0.07] \\
-0.63[-1.06 ;-0.19]\end{array}$ & $\begin{array}{c}\text { Reference } \\
-1.41[-2.73 ;-0.09] \\
-0.74[-1.84 ; 0.40] \\
-1.29[-2.33 ;-0.25] \\
-1.02[-1.91 ;-0.13]\end{array}$ & $\begin{array}{c}\text { Reference } \\
-0.47[-1.30 ; 0.36] \\
-0.04[-0.73 ; 0.65] \\
-0.20[-0.86 ; 0.46] \\
-0.04[-0.62 ; 0.53]\end{array}$ & $\begin{array}{c}\text { Reference } \\
-1.49[-2.22 ;-0.77] \\
-0.41[-0.94 ; 1.12] \\
-1.19[-1.70 ;-0.69] \\
-0.98[-1.44 ;-0.51]\end{array}$ & $\begin{array}{c}\text { Reference } \\
-0.57[-1.49 ; 0.35] \\
-0.39[-1.09 ; 0.31] \\
-0.11[-0.78 ; 0.56] \\
0.21[-0.40 ; 0.83]\end{array}$ \\
\hline
\end{tabular}

Coefficients are derived from separate multilevel multiple models (with individuals clustered in countries and each independent variable (i.e. GDP, EU membership or type of system), adjusted for age, gender, education, disease duration and ever having had sick leave due to RA; analyses on the rheumatologist domains were adjusted for age, gender and work setting; GDP - Gross Domestic Product, EU - European Union

Anglo-saxon: UK, Ireland; Scandinavian: Denmark, Iceland, Sweden, Norway, Finland; Bismarkian: Austria, Belgium, Germany, France, Israel, Netherlands, Switzerland; Mediterranean: Cyprus, Greece, Italy, Portugal, Spain, Turkey; Post-communist: Albania, Bulgaria, Czech Republic, Croatia, Estonia, Georgia, Hungary, Latvia, Lithuania, Poland, Romania, Russian Federation, Serbia, Tajikistan, Slovak Republic, Slovenia

sthe higher score the more positive are the perceptions

Statistically significant $(p<0.05)$ regression estimates are in bold 


\section{Discussion}

To our knowledge, this is the first study to provide an extended overview of systems to support work or work disability in RA. With regard to the system rules and regulations, i.e. availability and affordability of work disability arrangements, a large heterogeneity across countries was observed for most regulations including income compensation. While research and judgement on which system is preferable is complex and beyond the aim of this study, it is striking that a person with RA, upon becoming disabled, will face very different perspectives on future work participation, depending on his/her country of residence. Only in a minority of countries work re-integration plans were obligatory before starting a procedure towards WD.

Despite important variation, we could not detect patterns explaining differences in the formal rules and regulations of the SS systems, with only few exceptions. We could not find support to our first hypothesis that lower GDP countries would have stricter rules around work disability or lower relative income substation when WD is granted, nor did we find any common features of systems in lower GDP countries or EU vs non-EU member states. Moreover, the rules of the SS system did not seem to have a large role in explaining differences in WD in RA. Earlier research - mainly in general population and above 50 years of age in a number of European countries and dating back to 2007 suggested that the national SS system and, in particular, generosity of benefits, explains a large proportion of between country variations in disability rates.[25] We could not reproduce these results in our study among patients with $\mathrm{RA}$. It is worth noting that data on work disability rates in RA was available for 21 countries only and collected almost a decade earlier, and was based on a sample of patients rather than national statistics.[16] In contrast to findings on formal regulations (availability and affordability), patients' and rheumatologists' perceptions of systems to support persons with RA encountering work restrictions (acceptability), showed an apparent variation according to the type of the social security system: the Scandinavian and Bismarkian employment support and social security system consistently appeared to most adequately meet the expectations of patients and rheumatologists regarding remaining at work and application for a work disability pension. At the same time, little differences were related to country's wealth or EU-membership, and only a weak signal suggested that rheumatologists in lower income countries are more confident in their role to support patient in work disability issues. The 
latter may indicate that rheumatologists in low income countries interact more intensively with patients on these issues and accept work disability questions as part of their responsibility. Alternatively, other cultural and system factors could be considered, e.g. patients referring to other professionals within the system_such as primary care or state agencies) for advice and support regarding work disability decisions.[26] Overall, a lack of standardisation in the decision-making process on work disability was reported by nearly three quarters of the rheumatologists, and reinforces earlier calls for efforts for standardisation and homogenisation.[27] Therefore, our third hypothesis that levels of satisfaction with the system is higher in EU member states and higher GDP countries was not supported by the available data, while initial insight was generated with respect to the social security system type where no hypotheses were formed a priori.

This study has some notable limitations. First, the complexity of access to social security related to work and disability is hard to capture with a questionnaire that unavoidably simplifies reality. In absence of a validated tool to measure formal social security regulations and perceptions around the system performance, we used self-developed questionnaires. These happened in intensive collaboration with several international experts on work participation studies. We did not follow a formal translation procedure and the decision and responsibility to translate was left with the $\mathrm{PI}$, however, the translations were double-checked by patient partners. While government authorities would be the most knowledgeable parties to enquire about rules and regulations, the feasibility issues around establishing a direct contact with agencies from $>30$ European countries, in different languages, and where they likely have little or no interest to contribute to a research project, led us to choose for the well-established network of rheumatologists active in research was approached instead. To improve data quality and accuracy, Pls were encouraged to seek help from other experts in their country; the summary was sent to available patient partners for a face-validity check of data in their country, and the results showed a good agreement. It is emphasized in the discussion that this study has an explorative nature and limited conclusions should be drawn.

We have to acknowledge that application of the formal rules and regulations can differ substantially from the formal rules, and hence patients in countries with similar rules could potentially have different experiences when rules are applied. For example, partial work disability (which formally implies that a patient can work part of the time) may be a barrier 
to any employment in some countries and thus perceived differently compared to countries where patients deemed partially disabled are able to use their right for part-time work. We have attempted to get the initial insight on this through the surveys among patients and rheumatologists. Furthermore, it was challenging to select the best countrylevel characteristics that should be related to indicators of access. On this line, the social security system taxonomy used[23] is most likely an oversimplification of the complex systems that are constantly changing and developing, but to our knowledge, no alternative taxonomy exists. Recourse to (official) disability is a complex construct in which social security has a limited role. Alternative approaches to gain insight into international variation should be considered to study the performance and impact or social security systems. One of the potentially promising method could consist of series of clinical vignettes. By considering the rules and regulations that are to be applied to a hypothetical patient with given characteristics in terms of disease, work situation and disability, as well as attitudes and values about role of work in life and society, countries could be compared and further classified.

Despite limitations, we hereby present the first attempt to understand whether patterns in regulations can be found to help to understand differences in employment, sick leave and work disability. We found differences in regulations and income substitution that are challenging our perceptions of equity and call for further research to justify them or for efforts to define the acceptable standards. Cost-effectiveness models in RA often count with an improvement of work disability in parallel with the improvement of the health status of the patient (e.g. HAQ based Markov models). Our study suggests that large variations exist between countries regarding regulations of short term absence or work disability that can affect their chances to return to work, a point to consider in economic evaluations. Although we did not find clear relations between regulations and work participation rates, we should keep in mind the previous research that suggested that patients continue to work at different health status across countries.[16] As system type appears to have a rather limited impact on regulations and perceptions on them, this might indicate that interventions to support work retention in patients with RA could in principle be considered irrespective of social security system. Moreover, the ageing of the population worldwide urges policy-makers to increase the age of retirement, meaning that people with RA will also be expected to work longer in the years to come. 
In conclusion, we observed large heterogeneity in rules and regulations of social security systems across Europe in relation to work disability of patients with RA, and these cannot be explained by existing welfare regimes, EU membership or country's wealth. These differences call for a platform to consider harmonization of policies for patients with RA who experience restrictions in work participation. While remarkably little differences of patients' and rheumatologists' perceptions are related to country's wealth and membership in EU, Scandinavian employment support and social security system appears to most adequately meet the expectations of patients and rheumatologists regarding endurable work participation and access to work disability pension.

\section{Disclosure}

Authors declare no conflict of interest.

Competing interests

Authors declare no conflict of interest.

\section{Contributorship}

PP, SR and AB conceived the study idea, FG, MP, FS, TS, MdW, AW, AZ contributed to the protocol and conceptualisation, AK advised on statistical analyses, DA, FB, IB, SB, KC, PC, RC, ED, ND, AF, OFG, GG, NG, PG, MH, IJ, JV, XJ, MKov, MKull, LCM, MM, SMP, IN, ON, IFP, $\mathrm{KP}, \mathrm{BR}, \mathrm{HR}, \mathrm{FS}, \mathrm{GS}$, IS, NS, PS, RS, SSok, SShuk, AT, MT, TU, SV collected data. All authors read the manuscript draft and approved the final submission.

\section{Acknowledgements}

We would like to acknowelde our patient research partners for their invaluable contribution at different stages of this project: Eva Decantere (Belgium), Boryana Boteva (Bulgaria), Kouloumas Marios (Cyprus), Alena Slamova (Czech Republic), Lena Anderson (Denmark), Tiina Jasinski (Estonia), Sonia Trope (France), Dieter Wiek (Germany), Mary Vella (Malta), Margôt Bakkers (the Netherlands), Dragan Chichikj (Macedonia), Gerd Jenny Aanerud (Norway), Jolante Grygielska (Poland), Elsa Mateus (Portugal), Zabalan Codruta (Romania), Polina Pchelnikova (Russia), Petra Bednarova (Slovakia), Aladar Belec (Slovenia), Laly Alcaide (Spain), Ayhan Dinc (Turkey), Pamela Richards (UK), National Rheumatoid Arthritis Society (NRAS, UK).

Funding info 


\section{Figure 1. Study design.}

This study was partially funded by Abbvie in the context of Fit for Work initiative. SV was supported by Versus Arthritis (grant numbers 20385) and NIHR Manchester Biomedical Research Centre.

\section{Patient and Public Involvement}

Patient partners have been involved in design of the study, as well in developing and testing survey instruments in different countries.

\section{Ethical approval information}

The project has been approved by Maastricht Ethical Committee. Principal Investigators in each country were responsible for local ethical approvals, where necessary.

\section{Data sharing statement}

\section{Most of the data is provided in the online appendices. Further details can be provided upon request.}

\section{References}

1. Innala L, Berglin E, Moller B, et al. Age at onset determines severity and choice of treatment in early rheumatoid arthritis: a prospective study. Arthritis Res Ther 2014;16:R94

2. Cross M, Smith E, Hoy D, et al. The global burden of rheumatoid arthritis: estimates from the global burden of disease 2010 study. Ann Rheum Dis 2014;73:1316-22

3. Eriksson JK, Neovius M, Ernestam S, et al. Incidence of rheumatoid arthritis in Sweden: a nationwide population-based assessment of incidence, its determinants, and treatment penetration. Arthritis Care Res (Hoboken) 2013;65:870-8

4. Matcham F, Scott IC, Rayner L, et al. The impact of rheumatoid arthritis on quality-of-life assessed using the SF-36: a systematic review and meta-analysis. Semin Arthritis Rheum 2014;44:123-30

5. Lenssinck ML, Burdorf A, Boonen A, et al. Consequences of inflammatory arthritis for workplace productivity loss and sick leave: a systematic review. Ann Rheum Dis 2013;72:493-505

6. Backman CL. Employment and work disability in rheumatoid arthritis. Curr Opin Rheumatol 2004;16:148-52

7. Sokka T. Work disability in early rheumatoid arthritis. Clin Exp Rheumato/ 2003;21:S71-4

8. Tiippana-Kinnunen T, Paimela L, Peltomaa R, et al. Work disability in Finnish patients with rheumatoid arthritis: a 15-year follow-up. Clin Exp Rheumatol 2014;32:88-94

9. Scott DL, Smith C, and Kingsley G. What are the consequences of early rheumatoid arthritis for the individual? Best Pract Res Clin Rheumatol 2005;19:117-36 
10. Wolfe F, Michaud K, Choi HK, et al. Household income and earnings losses among 6,396 persons with rheumatoid arthritis. J Rheumatol 2005;32:1875-83

11. Angelini V, Cavapozzi D, and Paccagnella O, Dynamics of work disability reporting in Europe, in 'Marco Fanno' Working Paper (104). 2009: University of Padua.

12. OECD, Sickness, Disability and Work: Breaking the Barriers A Synthesis of Findings across OECD Countries. 2010.

13. Borsch-Supan A, Brugiavini A, and Croda E. The Role of Institutions and Health in European Patterns of Work and Retirement. J Eur Soc Policy 2009;19:341-358

14. Chung $\mathrm{CP}$, Sokka T, Arbogast PG, et al. Work disability in early rheumatoid arthritis: higher rates but better clinical status in Finland compared with the US. Ann Rheum Dis 2006;65:1653-7

15. van der Zee-Neuen A, Putrik P, Ramiro S, et al. Large country differences in work outcomes in patients with RA - an analysis in the multinational study COMORA. Arthritis Res Ther 2017;19:216

16. Sokka $\mathrm{T}$, Kautiainen $\mathrm{H}$, Pincus $\mathrm{T}$, et al. Work disability remains a major problem in rheumatoid arthritis in the 2000s: data from 32 countries in the QUEST-RA study. Arthritis Res Ther 2010;12:R42

17. European Commission, Reducing health inequalities in the European Union, in Luxembourg: Publications Office of the European Union. 2010.

18. de Croon EM, Sluiter JK, Nijssen TF, et al. Work ability of Dutch employees with rheumatoid arthritis. Scand J Rheumatol 2005;34:277-83

19. Odegard S, Finset A, Kvien TK, et al. Work disability in rheumatoid arthritis is predicted by physical and psychological health status: a 7-year study from the Oslo RA register. Scand J Rheumatol 2005;34:441-7

20. McIntyre D, Thiede M, and Birch S. Access as a policy-relevant concept in low- and middleincome countries. Health Econ Policy Law 2009;4:179-93

21. Putrik P, Ramiro S, Kvien TK, et al. Inequities in access to biologic and synthetic DMARDs across 46 European countries. Annals of the rheumatic diseases 2014;73:198-206

22. IMF. 2011; World Economic Outlook Database (http://www.imf.org/external/pubs/ft/weo/2011/02/weodata/index.aspx). June, 2014.

23. Alvarez-Galvez J, Rodero-Cosano, M.L., García-Alonso, C. et al. Changes in socioeconomic determinants of health: comparing the effect of social and economic indicators through European welfare state regimes. J Public Health 2014;22:305

24. Sokka T, Kautiainen H, Toloza S, et al. QUEST-RA: quantitative clinical assessment of patients with rheumatoid arthritis seen in standard rheumatology care in 15 countries. Ann Rheum Dis 2007;66:1491-6

25. Börsch-Supan A, Work disability, health and incentive effects. MEA Discussion Paper (07135). 2007: University of Mannheim.

26. Ledingham JM, Snowden N, Rivett A, et al. Patient- and clinician-reported outcomes for patients with new presentation of inflammatory arthritis: observations from the National Clinical Audit for Rheumatoid and Early Inflammatory Arthritis. Rheumatology (Oxford) 2017;56:231-238

27. Barth J, de Boer WE, Busse JW, et al. Inter-rater agreement in evaluation of disability: systematic review of reproducibility studies. BMJ 2017;356:j14 


\section{Online supplementary material}

\section{Contents}

Online Supplementary Text S1. Questionnaire administered to Principal Investigators (one

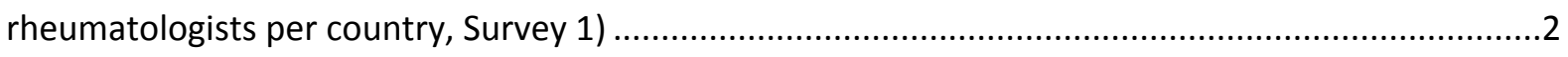

Online Supplementary Text S2. Questionnaire for rheumatologists (Survey 2)................................23

Online Supplementary Text S3. Questionnaire for patients (Survey 3) ............................................27

Online Supplementary Table S1. Social security system arrangements in case of sick leave due to RA

Online Supplementary Table S2. Social security system arrangements in case of work disability due to RA

Online Supplementary Table S3. Regulations to prevent work disability and help staying at work for people with RA

Online Supplementary Table S4. Patients' and rheumatologists' perceptions across several domains according to (1) GDP per capita PPP (2) EU membership status (3) Type of social security system ....43 


\section{Online Supplementary Text S1a. Questionnaire administered to Principal Investigators (one rheumatologists per country, Survey 1)}

\section{Research project: "Access to social security benefits in case of sick leave or work disability in rheumatoid arthritis across European countries".}

The aim of the project is:

1. To gain insight into the differences in access to social security benefits in case of sick leave and work disability for patients with rheumatoid arthritis (RA), in terms of eligibility criteria, and

2. To compare the level of income substitution and impoverishment in different countries across Europe.

\section{Questionnaire to be completed by the principal investigator of each country}

The principal investigator of each country is asked to complete the questionnaire below. This questionnaire consists of five parts:

1. Facilities to allow continuing participation in work force and to prevent work disability

2. Regulations in case of sick leave (short-term work disability)

3. Regulations in case of long-term work disability

4. Facilities to return to work after a period of long-term disability

5. Regulations in case of disability for self-employed persons

\section{Co-authorship rules}

The Principal Investigator will be a co-author in case the journal does not set strict limitations for the number of authors. We have confirmed with ARD (journal of first choice) that $>40$ authors is allowed given that author provides sufficient contribution. In this case the co-author will become coresponsible for the information published. More than 40 countries are invited to participate. Therefore, please note that due to restrictions from journals regarding the maximum number of authors, we can include only one person per country as a co-author. If the journal allows, one additional person who has substantially contributed to the data collection process could be included as a member of the working group and up to two persons can be acknowledged at the end of the manuscript.

Instructions on how to complete the questionnaire 
Completion of some parts of the questionnaire may require inquiring experts in your country who possess the specific knowledge on regulations in respect of work disability allowances. We encourage you to make all the efforts to obtain the most reliable information, which will likely involve contacting other people you consider appropriate. We also ask you to provide the official sources of information of the obtained data, as those are essential for publication of the final manuscript.

\section{Focus of the questionnaire}

Please, keep in mind that all the questions are related to work disability due to rheumatoid arthritis and patients who have an employment contract. In view of increasing rates of self-employed in some countries, we have added a section with a few general questions on work disability rules for selfemployed patients at the end of the questionnaire.

\section{Further recommendations on completing the questionnaire}

Due to expected large differences between systems, we attempt to make a universally applicable questionnaire to cover the general topics. It can be that some questions are not applicable to the situation in your country. After each question, there is always space left for additional comments from your side, and we invite you to provide additional comments for us to understand the local system better. Always think about the most general and most applicable case. Please, disregard variations and exceptions (for example, across regions or minority groups) or, if in doubt, describe them in the field for additional comments). In case it seems that the questions do not cover sufficiently the situation in your country, please indicate this in your comments. We will be in contact with Principal Investigator and adjust the questionnaire with country specific additions, if needed.

\section{$\underline{\text { Paper and online versions of the questionnaire }}$}

It is also possible to fill in this questionnaire online. Individual link to the survey is available in the invitation letter you have received. Questions are identical in both versions of the survey, and either of two is enough to complete. Should you have difficulties in accessing the online survey, please, let us know as soon as possible.

\section{$\underline{\text { Contact information }}$}

Polina Putrik, e-mail: polina.putrik@gmail.com 


\section{Q1 Country}

Please, indicate for which country are you completing the questionnaire:

Country name: 


\section{Definitions and terms}

Please note, that unless explicitly stated otherwise, the questions are always referring to patients with RA.

For purposes of our project (and based on collected knowledge about different social security systems), we feel we should distinguish two situations in which a patient cannot continue working because of RA:

1. Sick leave (also referred to as short-term work disability), which is absence from work due to disease starting from day 1 of the disease-related absence with a prospect to go back to the same work place after recovery.

2. Long-term work disability, which implies permanent disability to work, which generally follows a period of sickness (short-term work disability). In this case, contract with employer is usually no longer in place

\section{Additional terminology}

Degree (\%) of work disability refers to level of incapacity to work due to physical and/or mental reasons.

Degree (\%) of work ability is an alternative measure of work disability that is being used in some countries. It relates to the extent a person is able to perform work activities compared to a nondisabled person.

Q2

Are these two definitions (sick leave and long-term work disability) similar to the definitions used in your country?

\section{Yes}

No, please explain

Additional comments 


\section{Structure of the questionnaire:}

Part 1. Regulations to prevent work disability and help staying at work for people with RA ..............6

Part 2. Short- and long-term work disability regulations ................................................................

Part 2.1 Regulations related to sick leave (short-term work disability): system-related requirements .8

Part 2.2. Regulations related to sick leave (short-term work disability): disease-related criteria for



Part 2.3. Regulations related to long-term work disability: system-related requirements.................12

Part 2.4. Regulations related to long-term work disability: disease related criteria ..........................14

Part 3. Arrangements to facilitate return to work for patients with health problems due to rheumatoid



Part 4. Regulations in case of work disability for patients who are self-employed.............................19






\section{Part 1. Regulations to prevent work disability and help staying at work for people with RA}

\begin{tabular}{|c|c|}
\hline \multirow[t]{4}{*}{ Q3 } & $\begin{array}{l}\text { Are there arrangements or facilities available for persons with RA that aim at retention of } \\
\text { the patient in paid employment? }\end{array}$ \\
\hline & $\begin{array}{l}\square \text { No } \\
\square \text { Yes. Please, choose below if applicable: } \\
\square \text { Vocational rehabilitation in the hospital } \\
\square \text { Vocational rehabilitation in private sector } \\
\square \text { Self-learning/self-management } \\
\square \text { Work coaches } \\
\square \text { Advice from occupational physician } \\
\square \text { Coaching (advice) from employment agency } \\
\square \text { Other, please, explain below }\end{array}$ \\
\hline & Source of information \\
\hline & Additional comments \\
\hline
\end{tabular}




\section{Part 2. Short- and long-term work disability regulations}

This part contains questions about system-related (part 2.1) and disease-related (part 2.2) criteria for obtaining the short-term work disability (sick leave) allowance.

For example, should a person be a citizen of the country to qualify, how long the person should be employed before he or she has a right for sick leave allowance, who is authorized to certify the sick leave, etc. Please, in part 2.1 fill in the information for both permanent and temporary contracts even if similar rules apply for both.

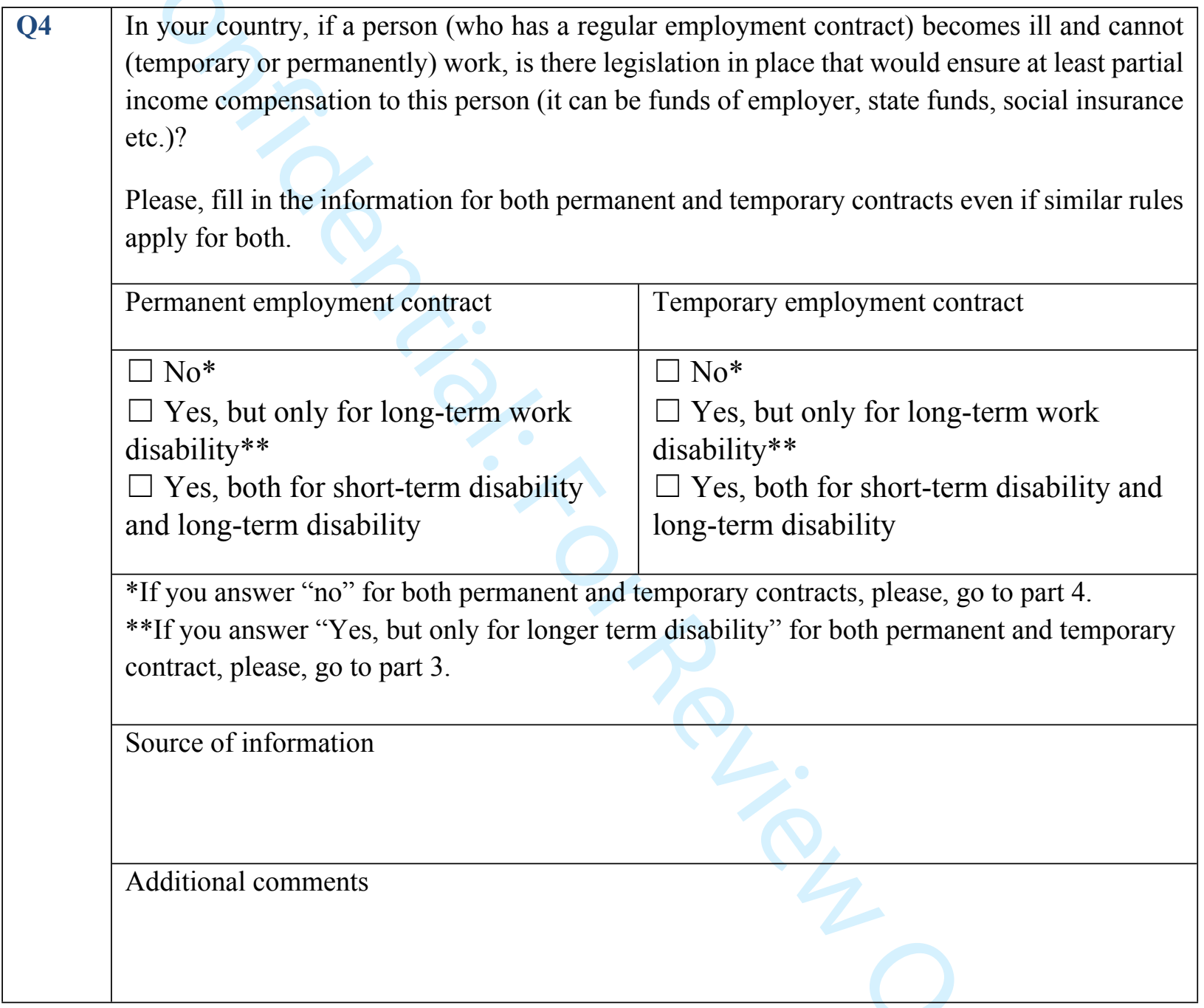


$\underline{\text { Part 2.1 Regulations related to sick leave (short-term work disability): system-related requirements }}$

\begin{tabular}{|c|c|c|}
\hline \multirow[t]{6}{*}{ Q5 } & \multicolumn{2}{|c|}{$\begin{array}{l}\text { Is there a requirement for a minimum number of years a person should reside in the country } \\
\text { to become eligible for sick leave allowance? }\end{array}$} \\
\hline & Permanent employment contract & Temporary employment contract \\
\hline & $\begin{array}{l}\square \text { No } \\
\square \text { Yes. Please, indicate the minimally } \\
\text { required period__ years } \\
\square \text { Yes, a person should be a citizen } \\
\square \text { Other. Please, explain }\end{array}$ & $\begin{array}{l}\square \text { No } \\
\square \text { Yes. Please, indicate the minimally } \\
\text { required period _ years } \\
\square \text { Yes, a person should be a citizen } \\
\square \text { Other. Please, explain }\end{array}$ \\
\hline & $\square$ Not applicable & $\square$ Not applicable \\
\hline & \multicolumn{2}{|l|}{ Source of information } \\
\hline & \multicolumn{2}{|l|}{ Additional comments } \\
\hline \multirow[t]{5}{*}{ Q6 } & \multicolumn{2}{|c|}{$\begin{array}{l}\text { Is there a requirement for the minimum length of official employment in order to be eligible } \\
\text { for a sick leave allowance? }\end{array}$} \\
\hline & Permanent employment contract & Temporary employment contract \\
\hline & $\begin{array}{l}\square \text { No } \\
\square \text { Yes, } \\
\square \text { Yes, } \\
\square \text { Yes, } \\
\square \text { workinths } \\
\square \text { Not applicable }\end{array}$ & 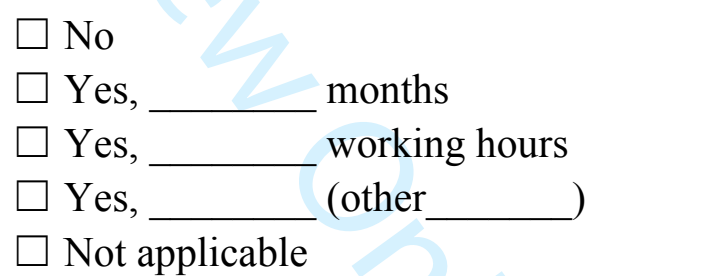 \\
\hline & \multicolumn{2}{|l|}{ Source of information } \\
\hline & Additional comments & \\
\hline
\end{tabular}




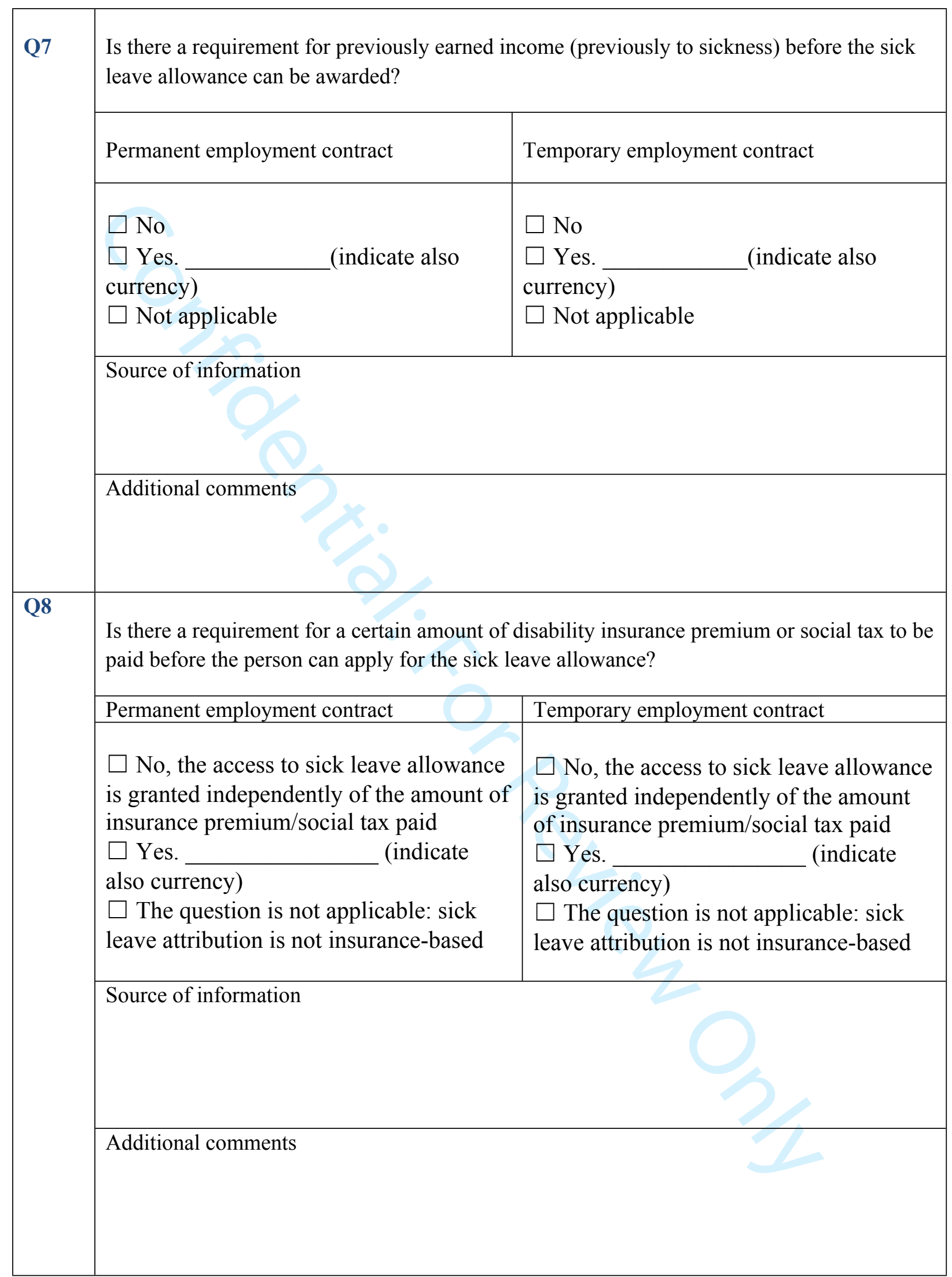


Part 2.2. Regulations related to sick leave (short-term work disability): disease-related criteria for eligibility

\begin{tabular}{|c|c|c|c|c|c|}
\hline \multirow[t]{4}{*}{ Q9 } & \multicolumn{5}{|c|}{$\begin{array}{l}\text { Is there a difference in sick leave compensation in case of permanent or temporary } \\
\text { employment contract? }\end{array}$} \\
\hline & \multicolumn{5}{|c|}{$\begin{array}{l}\square \text { No, there is no difference } \\
\square \text { Yes, the arrangement is different. Please, explain briefly }\end{array}$} \\
\hline & \multicolumn{5}{|l|}{ Source of information } \\
\hline & \multicolumn{5}{|l|}{ Additional comments } \\
\hline \multicolumn{6}{|c|}{$\begin{array}{l}\text { In case regulations for sick leave compensation are different for permanent and temporary contracts, } \\
\text { please refer in the following questions }(\mathbf{Q 1 0}-\mathbf{Q 1 4}) \text { to the conditions valid for those with a } \\
\text { permanent work contract }\end{array}$} \\
\hline \multirow[t]{11}{*}{ Q10 } & \multicolumn{5}{|c|}{$\begin{array}{l}\text { In case of RA, who can officially (i.e. legally) justify sick leave? We allow a possibility to } \\
\text { distinguish between the initial sick leave and prolonged sick leave, please, adjust the table } \\
\text { according to the reality in your country. It may be that not all columns have to be } \\
\text { completed. }\end{array}$} \\
\hline & \multirow{2}{*}{\multicolumn{2}{|c|}{ Qualification }} & $\begin{array}{l}\text { Initial sick } \\
\text { leave } \\
\text { (from day } \\
\quad \text { till day } \\
\text { ) }\end{array}$ & $\begin{array}{l}\text { First } \\
\text { prolongation } \\
\text { sick leave } \\
\text { (from } \\
\text { day_till } \\
\text { day_ }{ }^{*}\end{array}$ & $\begin{array}{l}\text { Second } \\
\text { prolongation } \\
\text { sick leave } \\
\text { (from day } \\
\text { till day_ _) }\end{array}$ \\
\hline & & & & \multicolumn{2}{|c|}{ *if applicable } \\
\hline & \multicolumn{2}{|c|}{ General practitioner (GP) } & $\square$ & $\square$ & $\square$ \\
\hline & \multicolumn{2}{|l|}{ Rheumatologist } & $\square$ & $\square$ & $\square$ \\
\hline & $\begin{array}{l}\text { Physician } \\
\text { (working for public } \\
\text { or private insurance } \\
\text { company/institute) }\end{array}$ & $\begin{array}{l}\text { Specialised in } \\
\text { occupational issues: } \\
\square \text { Yes } \\
\square \text { No }\end{array}$ & $\square$ & $\square$ & $\square$ \\
\hline & $\begin{array}{l}\text { Physician } \\
\text { (working for } \\
\text { employer) }\end{array}$ & $\begin{array}{l}\text { Specialised in } \\
\text { occupational issues: } \\
\square \text { Yes } \\
\square \text { No }\end{array}$ & $\square$ & $\square$ & $\square$ \\
\hline & \multicolumn{2}{|l|}{ Other } & $\square$ & $\square$ & $\square$ \\
\hline & \multicolumn{2}{|c|}{$\begin{array}{l}\text { Should not be justified (patient can report to } \\
\text { be sick and stay at home without anyone } \\
\text { certifying the reasons) }\end{array}$} & $\square$ & $\square$ & $\square$ \\
\hline & \multicolumn{5}{|l|}{ Source of information } \\
\hline & \multicolumn{5}{|l|}{ Additional comments } \\
\hline
\end{tabular}









\begin{tabular}{|l|l|}
\hline Q13 & $\begin{array}{l}\text { What is the maximum duration of sick leave allowed (e.g. eligible for compensation), } \\
\text { without entering long-term disability? }\end{array}$ \\
\hline $\begin{array}{l}\square \text { There is no maximum duration of sick leave specified } \\
\square \text { Continuous sick leave of _ months } \\
\square \text { Total sick leave of } \\
\square \text { Year } \\
\square \text { Other period }\end{array}$ \\
\hline $\begin{array}{l}\text { Source of information } \\
\text { Additional comments }\end{array}$ \\
\hline
\end{tabular}

$\underline{\text { Part 2.3. Regulations related to long-term work disability: system-related requirements }}$

Part 2.3 and 2.4 contain questions about system-related and disease-related criteria that apply when a person needs to receive long-term work disability allowance. Questions cover such topics as citizenship rules, length of employment contract, earned income etc. Further, you will be inquired about authorities that certify the disease and the fact that a person is not able to continue working and the criteria that would be taken into account when such decision is taken. Furthermore, questions about the levels of compensation (e.g. income substitution by the work disability pension) are asked. Please, in part 2.3 fill in the information for both permanent and temporary contracts even if similar rules apply for both.

\begin{tabular}{|c|c|c|}
\hline \multirow[t]{5}{*}{ Q14 } & \multicolumn{2}{|c|}{$\begin{array}{l}\text { Is there a requirement for a minimum number of years a person should reside in the country } \\
\text { to become eligible for long-term work disability allowance? }\end{array}$} \\
\hline & Permanent employment contract & Temporary employment contract \\
\hline & $\begin{array}{l}\square \text { No } \\
\square \text { Yes. Please, indicate the minimally } \\
\text { required period: _ years } \\
\square \text { Yes, a person should be a citizen } \\
\square \text { Not applicable }\end{array}$ & $\begin{array}{l}\square \text { No } \\
\square \text { Yes. Please, indicate the minimally } \\
\text { required period: } \\
\square \text { Yes, a person should be a citizen } \\
\square \text { Not applicable }\end{array}$ \\
\hline & \multicolumn{2}{|l|}{ Source of information } \\
\hline & \multicolumn{2}{|l|}{ Additional comments } \\
\hline Q15 & \multicolumn{2}{|c|}{$\begin{array}{l}\text { Is there a requirement for the minimum length of (official) employment in order to be eligible } \\
\text { for a long-term work disability allowance? }\end{array}$} \\
\hline
\end{tabular}







$\underline{\text { Part 2.4. Regulations related to long-term work disability: disease related criteria }}$

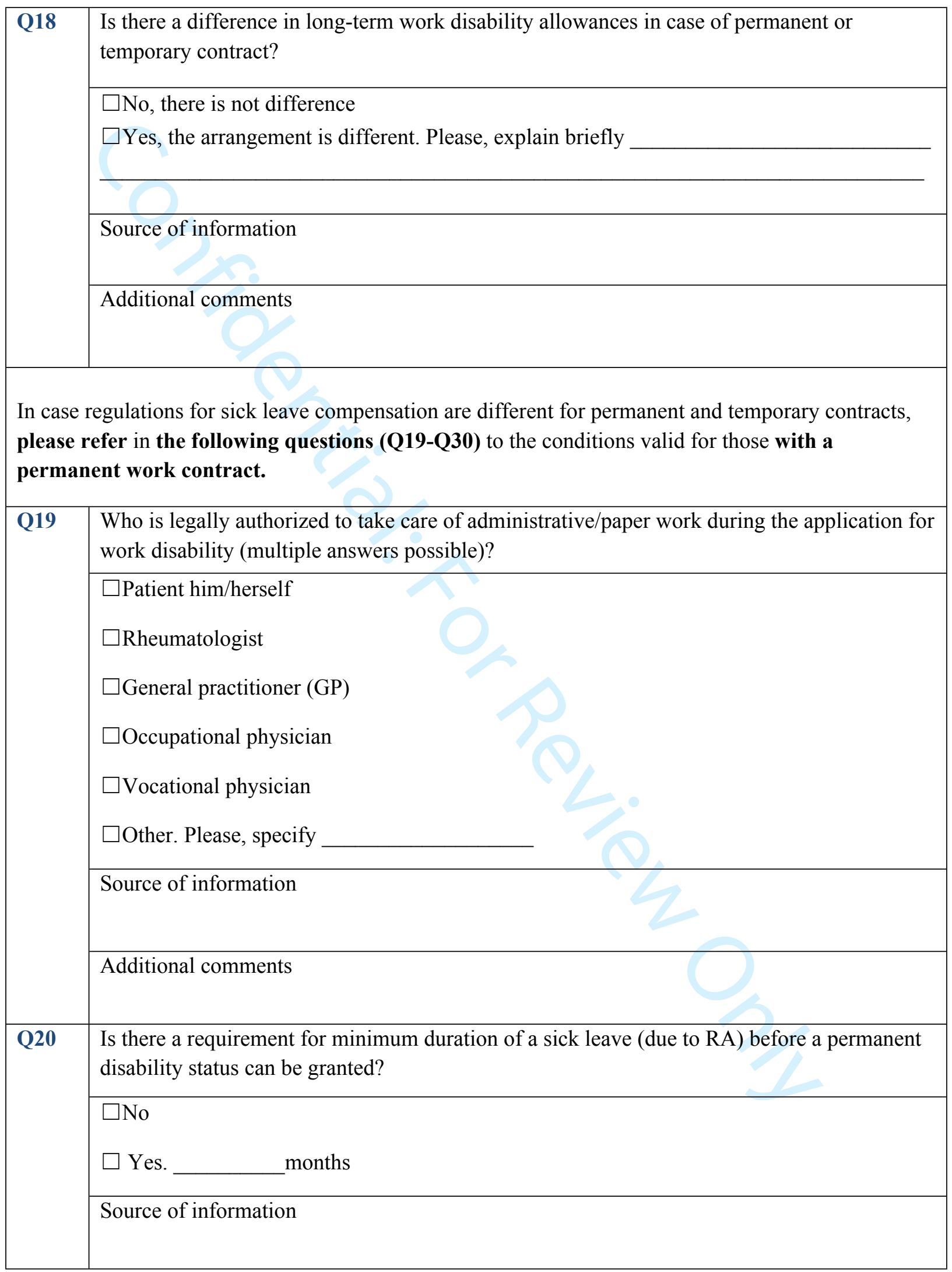




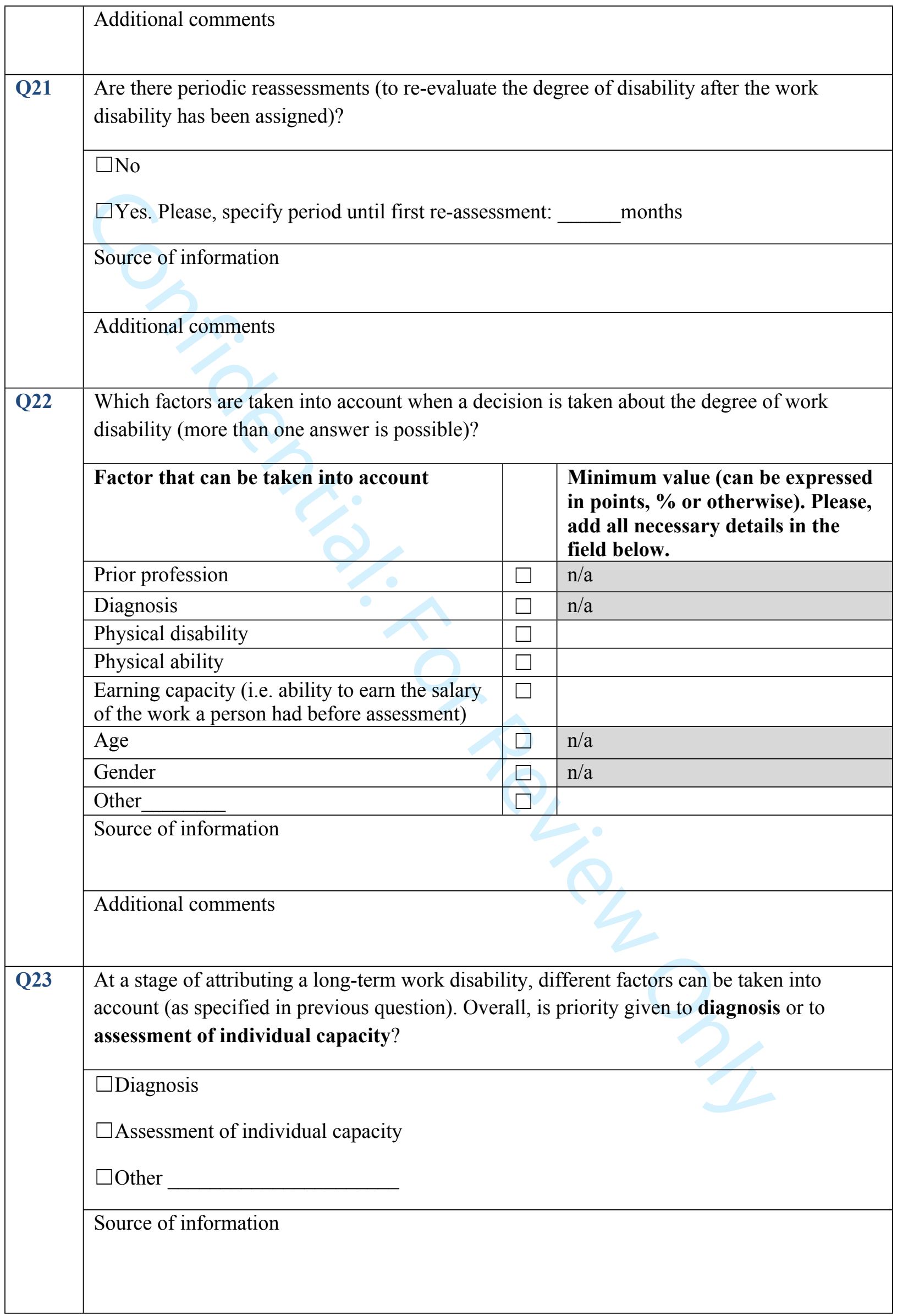




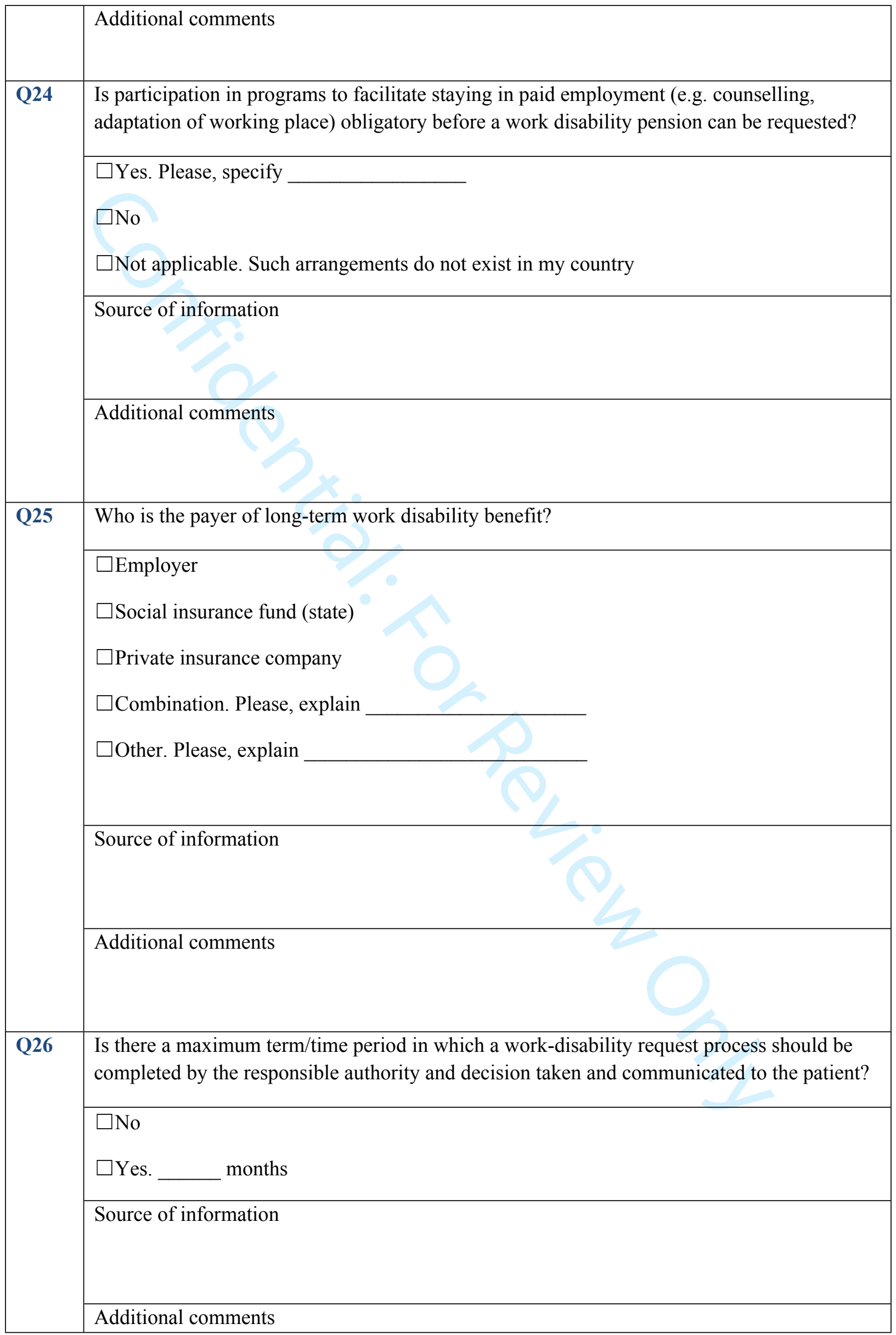




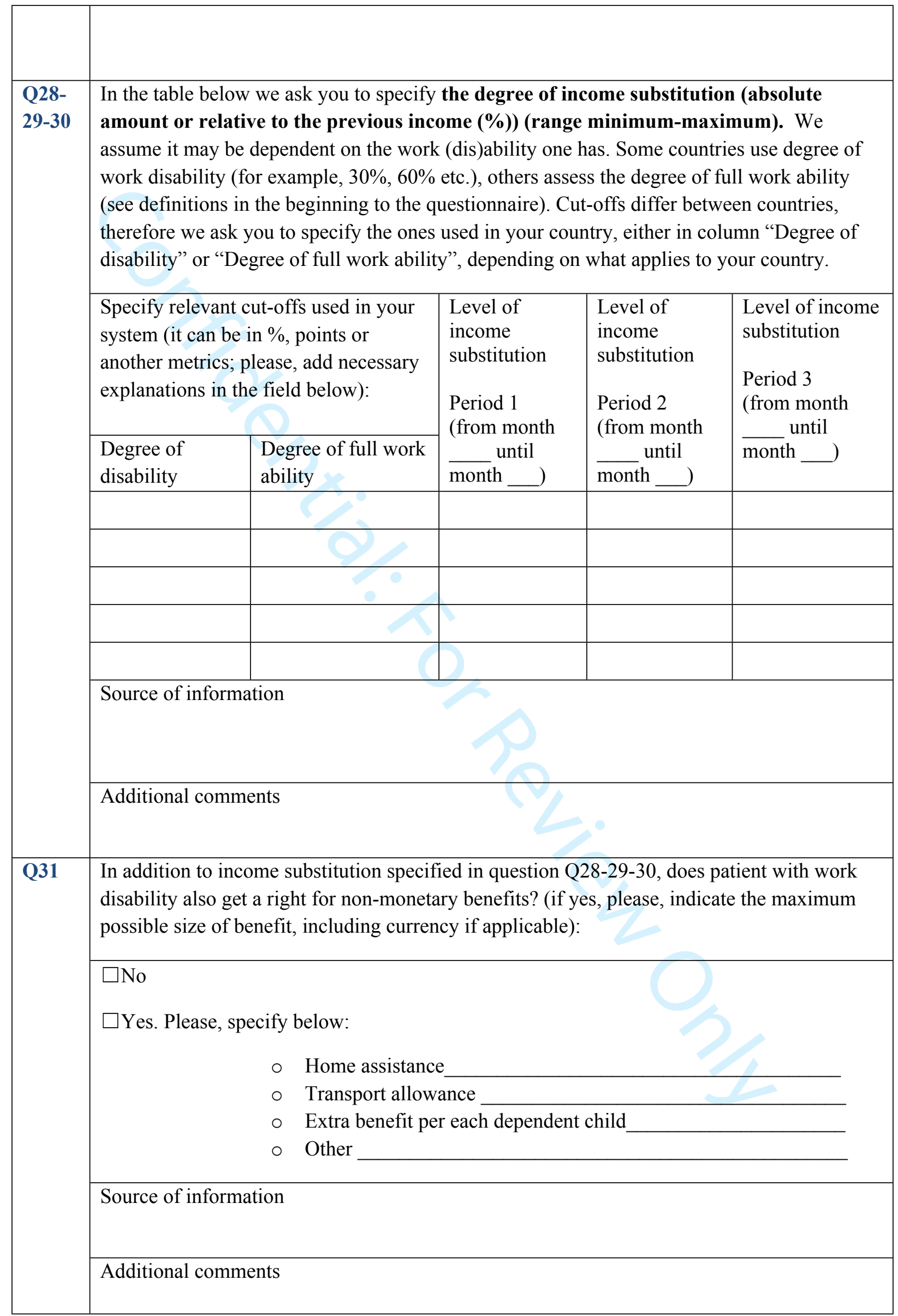


The questions of this section refer to the possibilities of continuing with work activity by a patient who was assigned a degree of long-term work disability, and possible regulations related to this process.

\section{Part 3. Arrangements to facilitate return to work for patients with health problems due to rheumatoid arthritis}

\begin{tabular}{|c|c|}
\hline \multirow[t]{4}{*}{ Q32 } & $\begin{array}{l}\text { Does the person who was assigned any degree of work disability have a right to work for a } \\
\text { monetary reward (it can be regular paid employment, occasional temporary work etc.)? }\end{array}$ \\
\hline & $\begin{array}{l}\square \text { No [go to question Q34] } \\
\square \text { Yes }\end{array}$ \\
\hline & Source of information \\
\hline & Additional comments \\
\hline \multirow[t]{4}{*}{ Q33 } & Does the right to work depend on the degree of work disability? \\
\hline & $\begin{array}{l}\square \text { No } \\
\square \text { Yes. Please, explain (including the relevant cut-offs for degrees of disability) }\end{array}$ \\
\hline & Source of information \\
\hline & Additional comments \\
\hline \multirow[t]{3}{*}{ Q34 } & $\begin{array}{l}\text { Are there any forms of societal support for people with a disability that would help them to } \\
\text { get employed (example can be special quotas for employers to hire people with disability, } \\
\text { subsidies for companies that employ people with disability, programs of additional education } \\
\text { etc.) }\end{array}$ \\
\hline & $\begin{array}{l}\square \text { No, this does not exist } \\
\square \text { Yes, but this does not apply for persons with RA } \\
\square \text { Yes, it exists, and also applies for persons with RA. Please, explain }\end{array}$ \\
\hline & Source of information \\
\hline
\end{tabular}




\section{Part 4. Regulations in case of work disability for patients who are self-employed}

Previous questions were referring to regulations concerning patients who are regularly employed (i.e. do not run their own business) at the moment of becoming ill. However, in some countries, the number of self-employed people is relatively large, and it is likely that other criteria for disability allowances apply for this group. Therefore, we added the section below with several general questions about work-disability regulations for self-employed population.

\begin{tabular}{|c|c|}
\hline \multirow[t]{4}{*}{ Q35 } & $\begin{array}{l}\text { Do people who are self-employed at the moment of sickness have a right for sick leave } \\
\text { compensation in case of temporary illness (in this question, we refer to state regulations, not } \\
\text { the voluntary insurance or individual savings)? }\end{array}$ \\
\hline & $\begin{array}{l}\square \text { No } \\
\square \text { Yes }\end{array}$ \\
\hline & Source of information \\
\hline & Additional comments \\
\hline \multirow[t]{4}{*}{ Q36 } & $\begin{array}{l}\text { Are people who are self-employed eligible for applying for long-term disability pension in } \\
\text { case their condition does not allow to continue working activities (in this question, we refer to } \\
\text { state regulations, not the voluntary insurance or individual savings)? }\end{array}$ \\
\hline & $\begin{array}{l}\square \text { No [Please, go to question Q38] } \\
\square \text { Yes. After___ months since the beginning of disease }\end{array}$ \\
\hline & Source of information \\
\hline & Additional comments \\
\hline \multirow[t]{2}{*}{ Q37 } & $\begin{array}{l}\text { You have answered that a person who is self-employed at the moment of sickness is eligible } \\
\text { for applying for long-term disability benefit in case his/her condition does not allow to continue } \\
\text { working activities. How is the amount of compensation (i.e. disability pension) determined? }\end{array}$ \\
\hline & $\begin{array}{l}\square \text { Based on previous income (please, specify the rules applied, including min and max) } \\
\square \text { Fixed amount } \quad \text { (indicate currency) }\end{array}$ \\
\hline
\end{tabular}




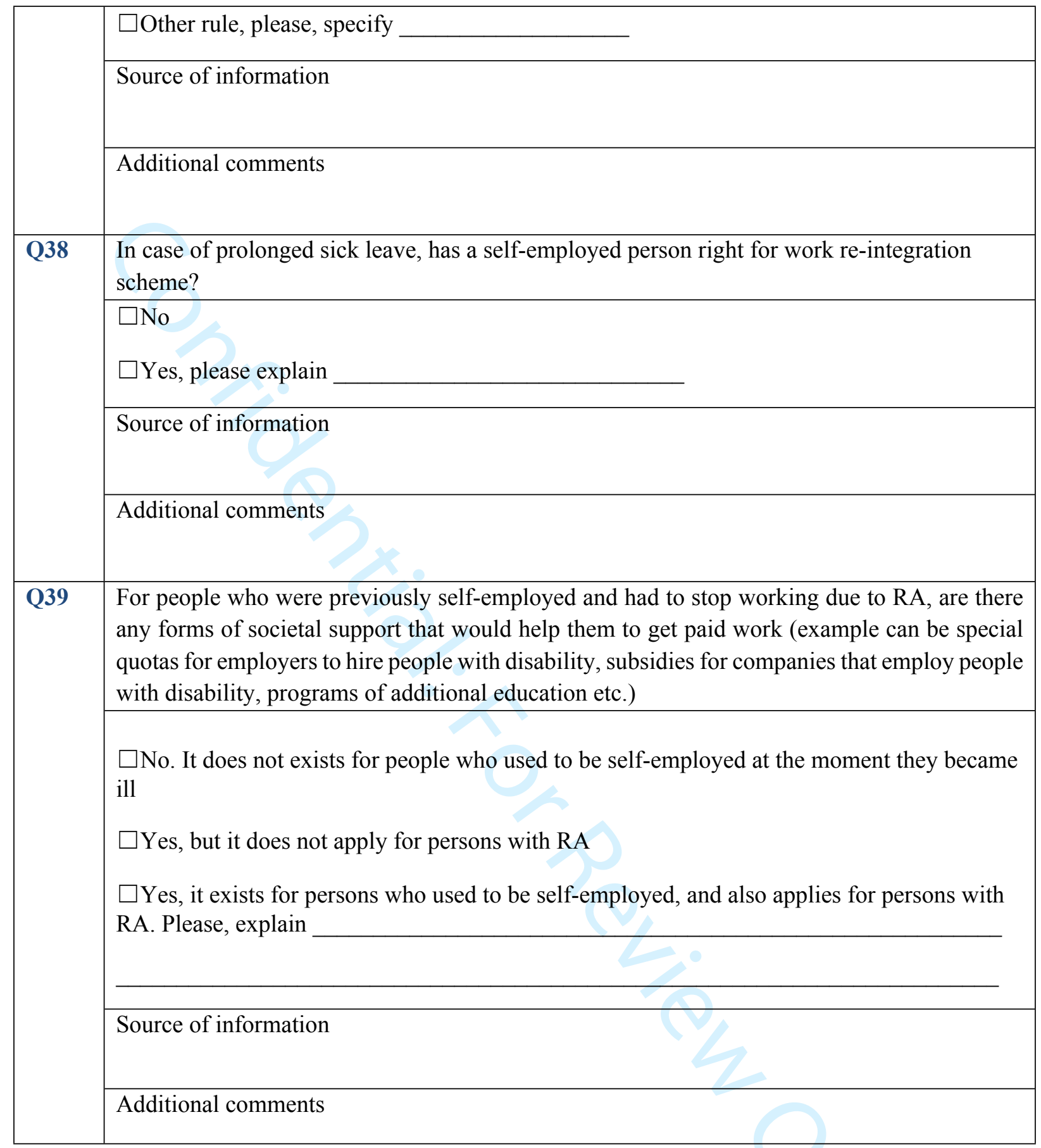




\section{Part 5. Other chronic diseases}

\begin{tabular}{|l|l|}
\hline Q40 & $\begin{array}{l}\text { Are the criteria/allowances specified in the previous sections of the questionnaire applicable to } \\
\text { other conditions than RA? }\end{array}$ \\
$\square$ No, only to RA \\
$\square$ Yes, to all rheumatologic conditions \\
$\square$ Yes, but only for (select the ones that are applicable): \\
$\square$ Psoriatic arthritis \\
$\square$ Osteoarthritis \\
$\square$ Ankylosis spondylitis/Spondyloarthritis \\
$\square$ Polyarthritis \\
$\square$ Chronic back pain \\
$\square$ Other chronic conditions, namely \\
\end{tabular}

Thank you for filling in the questionnaire! 
Online Supplementary Text S1b. Questionnaire administered to Principal Investigators (one rheumatologists per country, vignette scenario for income compensation)

\begin{tabular}{|c|c|c|c|}
\hline & $\begin{array}{l}\text { Female, } 50 \text { years old, citizen of } \\
\text { Has } 25 \text { years of work experien } \\
\text { Works in a large private compa }\end{array}$ & $\begin{array}{l}\text { e country } \\
40 \text { working hours per week }\end{array}$ & valent to full-time work) \\
\hline $\begin{array}{l}\text { Previous income during years preceding } \\
\text { illness }\end{array}$ & $\begin{array}{c}\text { Minimum salary per } 1 \text { month } \\
\end{array}$ & $\begin{array}{c}\text { Average salary per } 1 \text { month } \\
\end{array}$ & $\begin{array}{l}3 \text { times average salary per } 1 \text { month } \\
\end{array}$ \\
\hline $\begin{array}{l}\text { The patient was recently diagnosed with } \\
\text { very active RA and has not been able to } \\
\text { attend work job for } 6 \text { months (full-time sick } \\
\text { leave/short-term disability) } \\
\text { - Please, indicate actual amount of } \\
\text { income compensation that a person } \\
\text { will receive during } 6 \text { months period } \\
\text { (gross income, ie. before taxes, if any) }\end{array}$ & $\begin{array}{l}\text { Income compensation (per } 6 \text { months, as } \\
\text { described in scenario) }\end{array}$ & $\begin{array}{l}\text { Income compensation (per } 6 \text { months, as } \\
\text { described in scenario) }\end{array}$ & $\begin{array}{l}\text { Income compensation (per } 6 \text { months, as described } \\
\text { in scenario) }\end{array}$ \\
\hline $\begin{array}{l}\text { Patient has longstanding RA (10 years) and } \\
\text { was granted a year ago a permanent (long- } \\
\text { term) moderate work disability because of } \\
\text { physical incapacity to perform half ( } 50 \% \text { ) of } \\
\text { usual work activities) } \\
\text { - Please, indicate actual amount of } \\
\text { disability pension that a person would } \\
\text { have received during first year (gross } \\
\text { income, ie. before taxes, if any) }\end{array}$ & $\begin{array}{l}\text { Disability pension (per } 1 \text { year, as described in } \\
\text { scenario) }\end{array}$ & $\begin{array}{l}\text { Disability pension (per } 1 \text { year, as described in } \\
\text { scenario) }\end{array}$ & $\begin{array}{l}\text { Disability pension (per } 1 \text { year, as described in } \\
\text { scenario) }\end{array}$ \\
\hline
\end{tabular}




\begin{tabular}{|l|l|l|l|}
\hline $\begin{array}{l}\text { Patient has longstanding RA (10 years) and } \\
\text { was granted a year ago a permanent (long- } \\
\text { term) severe work disability, because of } \\
\text { physical incapacity to perform } 3 / 4(>75 \%) \text { of } \\
\text { usual work activities }\end{array}$ & $\begin{array}{l}\text { Disability pension (per 1 year, as described in } \\
\text { scenario) }\end{array}$ & $\begin{array}{l}\text { Disability pension (per 1 year, as described in } \\
\text { scenario) }\end{array}$ & $\begin{array}{l}\text { Disability pension (per 1 year, as described in } \\
\text { scenario) }\end{array}$ \\
$\begin{array}{l}\text { Please, indicate actual amount of } \\
\text { disability pension that a person would } \\
\text { have received during first year (gross } \\
\text { income, ie. before taxes, if any) }\end{array}$ & & \\
\hline
\end{tabular}




\section{Online Supplementary Text S2. Questionnaire for rheumatologists (Survey 2) \\ Perspective of rheumatologists}

\section{Acceptability of arrangements aimed at helping to stay at work or acquiring work disability pensions for patients with rheumatoid arthritis.}

This questionnaire is created to investigate opinion of rheumatologists about existing work support systems as well as regulations and attitudes in relation to work disability benefits for patient with rheumatoid arthritis in your country.

This study is part of a larger initiative to understand access to support systems and social security in case of problems to sustain paid work in patients with rheumatoid arthritis.

You have received this questionnaire because you are a rheumatologist and may have direct or indirect experience with the work participation and work disability benefits for patients with rheumatoid arthritis.

We are interested in having your opinion about different aspects related to work disability arrangements. We would like to ask you to give your opinion on the questions and statements below.

Please, note that the questionnaire is completely anonymous. Once you have completed the questionnaire you may choose among the following options to send it:

- You may send the completed version directly to the research team (Polina Putrik, polina.putrik@maastrichtuniversity.nl)

- You may return the questionnaire to the Principal Investigator in your country Dr. 
Q1 Please, indicate which country you are working in. *

*This survey is completely anonymous, and only generalizations on country level will be made during analyses stage.

1. Arrangements aimed at supporting to stay at work or acquiring work disability status for patients with rheumatoid arthritis

\begin{tabular}{|c|c|c|}
\hline Q2 & $\begin{array}{l}\text { The work related needs of patients } \\
\text { with RA are met by the (social } \\
\text { security) system in my country (i.e. } \\
\text { those who are able to work have } \\
\text { opportunity to continue working with } \\
\text { necessary adaptations) }\end{array}$ & $\begin{array}{l}\square \text { Yes, totally agree } \\
\square \text { Yes, agree } \\
\square \text { Neither agree nor disagree } \\
\square \text { No, disagree } \\
\square \text { Completely disagree } \\
\square \text { No opinion }\end{array}$ \\
\hline Q3 & $\begin{array}{l}\text { I feel that offering patient a chance } \\
\text { to stay at work (e.g. by developing a } \\
\text { work integration plan given patient's } \\
\text { reduced physical capacity) is as } \\
\text { important as offering a medical } \\
\text { treatment for his condition }\end{array}$ & $\begin{array}{l}\square \text { Yes, totally agree } \\
\square \text { Yes, agree } \\
\square \text { Neither agree nor disagree } \\
\square \text { No, disagree } \\
\square \text { Completely disagree } \\
\square \text { No opinion }\end{array}$ \\
\hline Q4 & $\begin{array}{l}\text { I feel confident and well-prepared } \\
\text { when it comes to supporting a } \\
\text { patient in maintaining/adjusting } \\
\text { his/her work duties so that he/she } \\
\text { can continue working }\end{array}$ & $\begin{array}{l}\square \text { Yes, totally agree } \\
\square \text { Yes, agree } \\
\square \text { Neither agree nor disagree } \\
\square \text { No, disagree } \\
\square \text { Completely disagree } \\
\square \text { No opinion }\end{array}$ \\
\hline Q5 & $\begin{array}{l}\text { The role of the rheumatologist in } \\
\text { my country is sufficient in the } \\
\text { process of decision making } \\
\text { regarding assigning the long-term } \\
\text { disability status for a patient }\end{array}$ & $\begin{array}{l}\square \text { Yes, totally agree } \\
\square \text { Yes, agree } \\
\square \text { Neither agree nor disagree } \\
\square \text { No, disagree } \\
\square \text { Completely disagree } \\
\square \text { No opinion }\end{array}$ \\
\hline
\end{tabular}




\begin{tabular}{|c|c|c|}
\hline Q6 & $\begin{array}{l}\text { If according to the rules in your } \\
\text { country you are the person who is } \\
\text { authorized to apply for work } \\
\text { disability pension on behalf of the } \\
\text { patient, how satisfied are you with } \\
\text { this task? }\end{array}$ & $\begin{array}{l}\square \text { I am not authorized to apply a and } \\
\text { feel comfortable with it } \\
\square \text { I am not authorized to apply a and } \\
\text { it is regrettable } \\
\square \text { I am authorized to apply and feel } \\
\text { comfortable with this role } \\
\square \text { I am authorized to apply and feel } \\
\text { uncomfortable with this role } \\
\square \text { Other }\end{array}$ \\
\hline Q7 & $\begin{array}{l}\text { If according to the rules in your } \\
\text { country you are the person who is } \\
\text { authorized to be involved in the } \\
\text { decision process for work } \\
\text { disability pension, how satisfied are } \\
\text { you with this task? }\end{array}$ & $\begin{array}{l}\square \text { I am not authorized to decide a } \\
\text { and feel comfortable with it } \\
\square \text { I am not authorized to decide a } \\
\text { and it is regrettable } \\
\square \text { I am authorized to decide and feel } \\
\text { comfortable with this role } \\
\square \text { I am authorized to decide and feel } \\
\text { uncomfortable with this role } \\
\square \text { Other }\end{array}$ \\
\hline Q8 & $\begin{array}{l}\text { The social security system is working } \\
\text { in a way that patients who need it } \\
\text { can get a work disability pension } \\
\text { (given the health status and personal } \\
\text { situation, such as family and work) }\end{array}$ & $\begin{array}{l}\square \text { Yes, totally agree } \\
\square \text { Yes, agree } \\
\square \text { Neither agree nor disagree } \\
\square \text { No, disagree } \\
\square \text { Completely disagree } \\
\square \text { No opinion }\end{array}$ \\
\hline Q9 & $\begin{array}{l}\text { How would you rate the level of } \\
\text { standardization (as opposite to } \\
\text { subjectivity) in the decision-taking } \\
\text { process regarding the long-term } \\
\text { disability? }\end{array}$ & $\begin{array}{l}\square \text { Very high level of standardization } \\
\text { (i.e. decisions are very objective) } \\
\square \text { High level of standardization (i.e. } \\
\text { decisions are objective) } \\
\square \text { Neither high nor low } \\
\square \text { Low level of standardization (i.e. } \\
\text { decisions are subjective) } \\
\square \text { Very low level of standardization } \\
\text { (i.e. decisions are very subjective) } \\
\square \text { No opinion }\end{array}$ \\
\hline Q10 & $\begin{array}{l}\text { I feel confident and well-prepared } \\
\text { when it comes to facilitating the } \\
\text { process of application for long-term } \\
\text { work disability benefit for my patients }\end{array}$ & $\begin{array}{l}\square \text { Yes, totally agree } \\
\square \text { Yes, agree } \\
\square \text { Neither agree nor disagree } \\
\square \text { No, disagree } \\
\square \text { Completely disagree } \\
\square \text { No opinion }\end{array}$ \\
\hline Q11 & $\begin{array}{l}\text { Taking all factors into account, } \\
\text { rheumatologists face a lot of }\end{array}$ & $\begin{array}{l}\square \text { Yes, totally agree } \\
\square \text { Yes, agree }\end{array}$ \\
\hline
\end{tabular}




\begin{tabular}{|l|l|l|}
\hline barriers when a patient should be & $\square$ Neither agree nor disagree \\
recognized as work disabled. & $\square$ No, disagree \\
& $\square$ Completely disagree \\
& $\square$ No opinion \\
\hline
\end{tabular}

\section{Background information about respondent}

This section contains questions about you and your work environment. This information will help us to understand and analyse your previous answers. All your responses will be anonymous, treated strictly confidential and used for the purpose of this study only.

\begin{tabular}{|c|c|c|}
\hline Q12 & What is your gender? & $\begin{array}{l}\square \text { Male } \\
\square \text { Female }\end{array}$ \\
\hline Q13 & What is your year of birth? & $19_{-}$ \\
\hline Q14 & $\begin{array}{l}\text { What best describes your current } \\
\text { work environment? (In case several } \\
\text { responses apply, please select your } \\
\text { main work environment only) }\end{array}$ & $\begin{array}{l}\square \text { University hospital (academic } \\
\text { setting) } \\
\square \text { Non-university (private or public) } \\
\text { hospital } \\
\square \text { Private practice } \\
\square \text { Other: }\end{array}$ \\
\hline Q15 & $\begin{array}{l}\text { How many years of work experience } \\
\text { as certified rheumatologist do you } \\
\text { have? }\end{array}$ & $\begin{array}{l}\square \text { Less than } 5 \text { years } \\
\square 6 \text { to } 10 \text { years } \\
\square 11 \text { to } 20 \text { years } \\
\square \text { More than } 20 \text { years }\end{array}$ \\
\hline Q16 & $\begin{array}{l}\text { What is the number of hours you } \\
\text { spend on patient care per week } \\
\text { (average)? }\end{array}$ & -- hours \\
\hline
\end{tabular}

Thank you for taking time to complete the questionnaire!

In case of questions, feel free to take direct contact with the research team: Polina Putrik, polina.putrik@maastrichtuniversity.nl or via telephone: (+31) 62478 7278 


\title{
Online Supplementary Text S3. Questionnaire for patients (Survey 3) \\ Perspective of patients
}

\section{Acceptability of arrangements aimed at helping to stay at work and acquiring work disability pensions for patients with RA.}

\begin{abstract}
This questionnaire is created to investigate
- the attitude of persons around you towards patients who have difficulties to work due to rheumatoid arthritis

and

- the acceptability of processes and regulations related to acquiring work disability status for patients with rheumatoid arthritis

You have received this questionnaire because you are a patient with rheumatoid arthritis and may have an opinion about different aspects related to work disability, such as possibilities to continue working despite of the disease, attitude of colleagues/society to patients with rheumatoid arthritis, application process to receive a disability pension, size of payments, etc. We would like to ask you to give your opinion on the questions and statements below.
\end{abstract}

Please, note that the questionnaire is completely anonymous. Once you have completed the questionnaire you may choose among the following options to send it:

- You may send the completed version directly to the research team (Polina Putrik, polina.putrik@maastrichtuniversity.nl)

- You may return the questionnaire to the Principal Investigator in your country (Dr. Principal Investigator, PLEASE REPLACE YOUR NAME HERE BEFORE PRINTING) 
Q1 Please, indicate which country you are living in*

*This survey is completely anonymous, and only generalizations on country level will be made during analyses stage.

1. Background information about respondent

\begin{tabular}{|c|c|c|}
\hline Q2 & What is your gender? & $\begin{array}{l}\square \text { Male } \\
\square \text { Female }\end{array}$ \\
\hline Q3 & What is your year of birth? & 19 \\
\hline Q4 & $\begin{array}{l}\text { When did you first experience symptoms } \\
\text { of rheumatoid arthritis (year)? }\end{array}$ & $-{ }_{-}-$(indicate year) \\
\hline Q5 & $\begin{array}{l}\text { When were you diagnosed with } \\
\text { rheumatoid arthritis? }\end{array}$ & ---- (indicate year) \\
\hline Q6 & $\begin{array}{l}\text { Have you ever had to be absent from } \\
\text { work due to your rheumatoid arthritis } \\
\text { (i.e. ask for a sick leave). If yes, please } \\
\text { indicate the longest period? }\end{array}$ & $\begin{array}{l}\square \text { No } \\
\square \text { Yes. Longest period } \\
\text { was__ days }\end{array}$ \\
\hline Q7 & $\begin{array}{l}\text { Has your RA ever caused substantial } \\
\text { problems with your work that made you } \\
\text { consider applying for work disability? }\end{array}$ & $\begin{array}{l}\square \text { No } \\
\square \text { Yes }\end{array}$ \\
\hline Q8 & $\begin{array}{l}\text { What is the highest level education you } \\
\text { have ever completed? }\end{array}$ & $\begin{array}{l}\square \text { Primary school diploma } \\
\square \text { Secondary school diploma } \\
\square \text { Higher than secondary } \\
\text { diploma (e.g. (in)completed } \\
\text { university diploma) }\end{array}$ \\
\hline Q9 & $\begin{array}{l}\text { What is your current work status? (check } \\
\text { all the boxes that apply) }\end{array}$ & $\begin{array}{l}\square \text { Paid work, part- or full-time } \\
\square \text { Do not have a paid work } \\
\text { (but NOT work disabled; e.g. } \\
\text { unemployed, student, } \\
\text { housewife, retired due to } \\
\text { age) } \\
\square \text { Certified work disability } \\
\text { (full or partial) }\end{array}$ \\
\hline
\end{tabular}


2. Stay at work arrangements for patients with rheumatoid arthritis

\begin{tabular}{|c|c|c|}
\hline Q10 & $\begin{array}{l}\text { Society (it can be, for example, colleagues } \\
\text { and/or employer) has an adequate } \\
\text { understanding of what I am able and not } \\
\text { able to do in a view of physical limitations } \\
\text { resulting from my RA }\end{array}$ & $\begin{array}{l}\square \text { Yes, totally agree } \\
\square \text { Yes, agree } \\
\square \text { Neither agree nor } \\
\text { disagree } \\
\square \text { No, disagree } \\
\square \text { Completely disagree } \\
\square \text { No opinion }\end{array}$ \\
\hline Q11 & $\begin{array}{l}\text { Society perceives me as less valuable } \\
\text { because of my RA (i.e. as being less able } \\
\text { to contribute to society). }\end{array}$ & $\begin{array}{l}\square \text { Yes, totally agree } \\
\square \text { Yes, agree } \\
\square \text { Neither agree nor } \\
\text { disagree } \\
\square \text { No, disagree } \\
\square \text { Completely disagree } \\
\square \text { No opinion }\end{array}$ \\
\hline Q12 & $\begin{array}{l}\text { I feel it is personally (not considering } \\
\text { financial benefit) rewarding to be able to } \\
\text { stay at work despite the physical limitation } \\
\text { caused by RA }\end{array}$ & $\begin{array}{l}\square \text { Yes, totally agree } \\
\square \text { Yes, agree } \\
\square \text { Neither agree nor } \\
\text { disagree } \\
\square \text { No, disagree } \\
\square \text { Completely disagree } \\
\square \text { No opinion }\end{array}$ \\
\hline Q13 & $\begin{array}{l}\text { I can get sufficient support in case I } \\
\text { prefer to stay at work despite of RA (e.g. } \\
\text { my working space can be adapted, as well } \\
\text { as work duties, schedules etc.) }\end{array}$ & $\begin{array}{l}\square \text { Yes, totally agree } \\
\square \text { Yes, agree } \\
\square \text { Neither agree nor } \\
\text { disagree } \\
\square \text { No, disagree } \\
\square \text { Completely disagree } \\
\square \text { No opinion }\end{array}$ \\
\hline Q14 & $\begin{array}{l}\text { My rheumatologist/ occupational } \\
\text { physician pays sufficient attention to } \\
\text { work related problems caused by RA (i.e. } \\
\text { makes suggestions/plans on how to } \\
\text { maintain the employment given my medical } \\
\text { condition) }\end{array}$ & $\begin{array}{l}\square \text { Yes, totally agree } \\
\square \text { Yes, agree } \\
\square \text { Neither agree nor } \\
\text { disagree } \\
\square \text { No, disagree } \\
\square \text { Completely disagree } \\
\square \text { No opinion }\end{array}$ \\
\hline
\end{tabular}

3. Work disability pension for patients with rheumatoid arthritis who are not able to continue working

\begin{tabular}{|l|l|l|}
\hline Q15 & $\begin{array}{l}\text { I perceive the level of work disability } \\
\text { pension due to RA as sufficient (i.e. I do }\end{array}$ & $\begin{array}{l}\square \text { Yes, totally agree } \\
\square \text { Yes, agree }\end{array}$ \\
\hline
\end{tabular}




\begin{tabular}{|c|c|c|}
\hline & $\begin{array}{l}\text { not feel forced to stay at work in order to } \\
\text { not loose income) }\end{array}$ & $\begin{array}{l}\square \text { Neither agree nor } \\
\text { disagree } \\
\square \text { No, disagree } \\
\square \text { Completely disagree } \\
\square \text { No opinion }\end{array}$ \\
\hline Q16 & $\begin{array}{l}\text { If I am not able to work due to my RA, I } \\
\text { can obtain work disability pension } \\
\text { without particular difficulties }\end{array}$ & $\begin{array}{l}\square \text { Yes, totally agree } \\
\square \text { Yes, agree } \\
\square \text { Neither agree nor } \\
\text { disagree } \\
\square \text { No, disagree } \\
\square \text { Completely disagree } \\
\square \text { No opinion }\end{array}$ \\
\hline Q17 & $\begin{array}{l}\text { In my opinion, persons who are } \\
\text { authorized to take decision regarding } \\
\text { work disability are knowledgeable about } \\
\text { the condition, i.e. know the natural } \\
\text { history of disease with flares, and the } \\
\text { impact of fatigue etc. }\end{array}$ & $\begin{array}{l}\square \text { Yes, totally agree } \\
\square \text { Yes, agree } \\
\square \text { Neither agree nor } \\
\text { disagree } \\
\square \text { No, disagree } \\
\square \text { Completely disagree } \\
\square \text { No opinion }\end{array}$ \\
\hline Q18 & $\begin{array}{l}\text { If I apply for work disability pension, my } \\
\text { personal situation (type of work, family } \\
\text { situation) will be taken into account } \\
\text { during the decision process }\end{array}$ & $\begin{array}{l}\square \text { Yes, totally agree } \\
\square \text { Yes, agree } \\
\square \text { Neither agree nor } \\
\text { disagree } \\
\square \text { No, disagree } \\
\square \text { Completely disagree } \\
\square \text { No opinion }\end{array}$ \\
\hline Q19 & $\begin{array}{l}\text { I think my gender makes a difference for } \\
\text { the process and decision regarding the } \\
\text { work disability pension due to RA }\end{array}$ & $\begin{array}{l}\square \text { Yes, totally agree } \\
\square \text { Yes, agree } \\
\square \text { Neither agree nor } \\
\text { disagree } \\
\square \text { No, disagree } \\
\square \text { Completely disagree } \\
\square \text { No opinion }\end{array}$ \\
\hline
\end{tabular}

4. Process of applying for official work disability for patients with rheumatoid arthritis who are not able to continue working

\begin{tabular}{|l|l|l|}
\hline Q20 & $\begin{array}{l}\text { I can easily access sufficient information } \\
\text { about requirements for application for } \\
\text { work disability pension for RA patients }\end{array}$ & $\square$ Yes, totally agree \\
& $\begin{array}{l}\square \text { Neither agree nor } \\
\text { disagree } \\
\square \text { No, disagree }\end{array}$ \\
\hline
\end{tabular}




\begin{tabular}{|c|c|c|}
\hline & & $\begin{array}{l}\square \text { Completely disagree } \\
\square \text { No opinion }\end{array}$ \\
\hline Q21 & $\begin{array}{l}\text { I can easily access sufficient information } \\
\text { about levels of compensation (monetary } \\
\text { and non-monetary benefits) in case of work } \\
\text { disability }\end{array}$ & $\begin{array}{l}\square \text { Yes, totally agree } \\
\square \text { Yes, agree } \\
\square \text { Neither agree nor } \\
\text { disagree } \\
\square \text { No, disagree } \\
\square \text { Completely disagree } \\
\square \text { No opinion }\end{array}$ \\
\hline Q22 & $\begin{array}{l}\text { In my opinion, the application process in } \\
\text { order to receive disability pension due to } \\
\text { RA is not difficult }\end{array}$ & $\begin{array}{l}\square \text { Yes, totally agree } \\
\square \text { Yes, agree } \\
\square \text { Neither agree nor } \\
\text { disagree } \\
\square \text { No, disagree } \\
\square \text { Completely disagree } \\
\square \text { No opinion }\end{array}$ \\
\hline Q23 & $\begin{array}{l}\text { In my opinion, I would be treated with } \\
\text { dignity and respect if I should apply for } \\
\text { work disability pension (or I have been } \\
\text { treated with dignity and respect when } \\
\text { applying for the pension) }\end{array}$ & $\begin{array}{l}\square \text { Yes, totally agree } \\
\square \text { Yes, agree } \\
\square \text { Neither agree nor } \\
\text { disagree } \\
\square \text { No, disagree } \\
\square \text { Completely disagree } \\
\square \text { No opinion }\end{array}$ \\
\hline Q24 & $\begin{array}{l}\text { In my opinion, there is sufficient } \\
\text { confidentiality throughout the process } \\
\text { of application to work disability pension } \\
\text { due to RA }\end{array}$ & $\begin{array}{l}\square \text { Yes, totally agree } \\
\square \text { Yes, agree } \\
\square \text { Neither agree nor } \\
\text { disagree } \\
\square \text { No, disagree } \\
\square \text { Completely disagree } \\
\square \text { No opinion }\end{array}$ \\
\hline Q25 & $\begin{array}{l}\text { I think that my rheumatologist has } \\
\text { sufficient role and influence in the } \\
\text { application process for work disability } \\
\text { pension due to RA }\end{array}$ & $\begin{array}{l}\square \text { Yes, totally agree } \\
\square \text { Yes, agree } \\
\square \text { Neither agree nor } \\
\text { disagree } \\
\square \text { No, disagree } \\
\square \text { Completely disagree } \\
\square \text { No opinion }\end{array}$ \\
\hline
\end{tabular}

In case of questions, feel free to take direct contact with the research team: Polina Putrik, polina.putrik@maastrichtuniversity.nl or via telephone: +31 62478 7278

Thank you for taking time to complete the questionnaire! 


\section{Online Supplementary Table S1. Social security system arrangements in case of sick leave due to RA}

\begin{tabular}{|c|c|c|c|c|c|c|c|c|c|c|c|c|}
\hline Country &  & $\begin{array}{l}\text { Length of } \\
\text { employme } \\
\text { nt to be } \\
\text { eligible for } \\
\text { Short - } \\
\text { term work } \\
\text { disability }\end{array}$ & 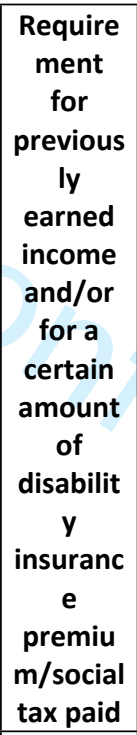 & $\begin{array}{c}\text { Who can justify sick } \\
\text { leave? }\end{array}$ & $\begin{array}{l}\text { When can } \\
\text { rheumatolog } \\
\text { ist be } \\
\text { involved in } \\
\text { certifying } \\
\text { sick leave }\end{array}$ & $\begin{array}{c}\text { When can } \\
\text { occupation } \\
\text { al therapist } \\
\text { be } \\
\text { involved in } \\
\text { certifying } \\
\text { sick leave }\end{array}$ & $\begin{array}{c}\text { When can } \\
\text { commission } \\
\text { of physicians } \\
\text { be involved } \\
\text { in certifying } \\
\text { sick-leave? }\end{array}$ & $\begin{array}{l}\text { Unpaid } \\
\text { first period } \\
\text { of sick } \\
\text { leave }\end{array}$ & $\begin{array}{l}\text { Maximum duration of } \\
\text { sick leave }\end{array}$ & $\begin{array}{c}\% \text { of } \\
\text { income } \\
\text { compensat } \\
\text { ion in first } \\
3 \text { months }\end{array}$ & $\begin{array}{c}\text { Period that } \\
\text { employer is } \\
\text { responsible } \\
\text { for (part of) } \\
\text { payment of } \\
\text { income } \\
\text { compensatio } \\
n\end{array}$ & $\begin{array}{l}\text { Is part of } \\
\text { this } \\
\text { period } \\
\text { shared } \\
\text { with } \\
\text { social } \\
\text { insuranc } \\
\text { e? }\end{array}$ \\
\hline $\begin{array}{c}\text { Albania }^{\mathrm{P}} \\
\text { (Non-EU, Low GDP) }\end{array}$ & No & $\begin{array}{c}\text { Not } \\
\text { specified }\end{array}$ & Yes & $\begin{array}{c}\text { GP, RHEUM, physician }{ }^{4} \\
\text { health commission }\end{array}$ & from day 1 & $\begin{array}{c}\text { from day } \\
60\end{array}$ & $\begin{array}{c}\text { from day } \\
180\end{array}$ & No & 6 months in one year & $70-80 \%$ & $\begin{array}{l}\text { first } 12 \\
\text { weeks }\end{array}$ & No \\
\hline $\begin{array}{c}\text { Austria }^{\mathrm{B}} \\
\text { (EU-15_High GDP) }\end{array}$ & No & $\begin{array}{c}\text { Not } \\
\text { specified }\end{array}$ & No & GP, RHEUM, physician ${ }^{5}$ & from day 1 & from day 1 & na & No & $\begin{array}{c}26 \text { or } 52 \text { weeks }^{7} \text {, with } \\
\text { max } 31 \text { days } \\
\text { interruption }\end{array}$ & $83 \%$ & day $4-12$ & No \\
\hline $\begin{array}{c}\text { Belarus }^{\mathrm{P}} \\
\text { (Non-EU, Low GDP) }\end{array}$ & Yes $^{1}$ & $\begin{array}{c}\text { Not } \\
\text { specified }\end{array}$ & No & GP, RHEUM, physician ${ }^{5}$ & from day 1 & na & na & No & $\begin{array}{c}120 \text { days } \\
\text { continuously or } 150 \\
\text { days intermittently } \\
\end{array}$ & $96 \%$ & $\begin{array}{l}\text { first } 6 \\
\text { months }\end{array}$ & No \\
\hline $\begin{array}{c}\text { Belgium }^{\mathrm{B}} \\
\text { (EU-15, High GDP) }\end{array}$ & No & $\begin{array}{c}1-6 \\
\text { months }\end{array}$ & No & GP, rheumat. & from day 1 & na & na & No & $\begin{array}{l}12 \text { months, max. } 3 \\
\text { months interruption }\end{array}$ & $60 \%$ & day $7-30$ & No \\
\hline $\begin{array}{c}\text { Bosnia\&Herzegovina }{ }^{P} \\
\text { (Non-EU, Low GDP) }\end{array}$ & No & $\begin{array}{c}\text { Not } \\
\text { specified }\end{array}$ & No & GP, rheumat. & from day 1 & na & na & No & 12 months & $90 \%$ & $\begin{array}{l}\text { first } 12 \\
\text { weeks }\end{array}$ & No \\
\hline $\begin{array}{c}\text { Bulgaria }^{\mathrm{P}} \\
\text { (EU-28_Low GDP) }\end{array}$ & No & $\begin{array}{l}>=6 \\
\text { months }\end{array}$ & No & $\begin{array}{l}\text { GP, hospital MD, medical } \\
\text { advisory committees, } \\
\text { territorial expert medical } \\
\text { commission }\end{array}$ & na & na & from day 30 & No & 18 months & $80 \%$ & first 3 days & No \\
\hline $\begin{array}{c}\text { Croatia }^{\mathrm{P}} \\
\text { (EU-28, Low GDP) }\end{array}$ & No & $\begin{array}{c}>=6 \\
\text { months }\end{array}$ & No & GP, physician ${ }^{5}$ & na & $\begin{array}{l}\text { from day } \\
365\end{array}$ & na & No & 12 months & $75 \%$ & first 42 days & No \\
\hline $\begin{array}{c}\text { Cyprus }^{\mathrm{M}} \\
\text { (EU-28, HighGDP) }\end{array}$ & No & $\begin{array}{c}>=6 \\
\text { months }\end{array}$ & Yes & GP, RHEUM, Physician ${ }^{6}$ & from day 1 & from day 1 & na & Yes, 3 days & 156 days & $57 \%$ & $\begin{array}{c}\text { first } 156 \\
\text { days }\end{array}$ & Yes \\
\hline
\end{tabular}




\begin{tabular}{|c|c|c|c|c|c|c|c|c|c|c|c|c|c|}
\hline 1 & $\begin{array}{c}\text { Czech Republic }^{P} \\
\text { (EU-28, High GDP) }\end{array}$ & No & $\begin{array}{c}\text { Not } \\
\text { specified }\end{array}$ & Yes & GP, physician ${ }^{5}$ & from day 1 & from day 1 & na & Yes, 3 days & 12 months & $57 \%$ & day $4-14$ & No \\
\hline $\begin{array}{l}2 \\
3\end{array}$ & $\begin{array}{c}\text { Denmark }^{\mathrm{S}} \\
\text { (EU-15, High GDP) }\end{array}$ & No & $\begin{array}{c}1-6 \\
\text { months }\end{array}$ & No & RHEUM, physician ${ }^{5}$ & from day 1 & na & na & No & 3 years & $100 \%$ & $\begin{array}{l}\text { first } 22 \\
\text { weeks } \\
\end{array}$ & Yes \\
\hline $\begin{array}{l}4 \\
5 \\
6 \\
7 \\
8\end{array}$ & $\begin{array}{c}\text { Eestonia }^{\mathrm{P}} \\
(\mathrm{EU}-28, \text { Low GDP) }\end{array}$ & No & $\begin{array}{c}1-6 \\
\text { months }\end{array}$ & No & GP, physician ${ }^{5}$ & from day 1 & $\begin{array}{c}\text { from day } \\
121\end{array}$ & na & Yes & $\begin{array}{l}\text { Continuous sick leave } \\
\text { of } 182 \text { days or total } \\
\text { sick leave of } 250 \text { days } \\
\text { (e.g. several periods } \\
\text { of sick leave) }\end{array}$ & $67 \%$ & day $4-8$ & No \\
\hline $\begin{array}{l}9 \\
10 \\
11\end{array}$ & $\begin{array}{c}\text { Finland }^{\mathrm{S}} \\
\text { (EU-15, High GDP) }\end{array}$ & No & $\begin{array}{c}\text { Not } \\
\text { specified }\end{array}$ & No & GP, RHEUM, physician ${ }^{6}$ & from day 1 & from day 1 & na & No & $\begin{array}{c}12 \text { months per } 2 \\
\text { years }\end{array}$ & $\begin{array}{c}\text { not } \\
\text { possible to } \\
\text { define } \%\end{array}$ & First 56 days & Yes \\
\hline $\begin{array}{l}12 \\
13 \\
14 \\
15\end{array}$ & $\begin{array}{c}\text { France }^{\mathrm{B}} \\
\text { (EU-15, High GDP) }\end{array}$ & No & $\begin{array}{c}1-6 \\
\text { months }\end{array}$ & Yes & GP, RHEUM & from day 1 & na & na & Yes, 3 days & 360 days per 3 years & $76 \%$ & $\begin{array}{c}\text { from day } 4, \\
\text { max. } 360 \\
\text { days per } 3 \\
\text { years } \\
\end{array}$ & Yes \\
\hline $\begin{array}{l}16 \\
17\end{array}$ & $\begin{array}{c}\text { Georgia }^{\mathrm{P}} \\
\text { (Non-EU, Low GDP) }\end{array}$ & na & $\begin{array}{c}\text { Not } \\
\text { specified }\end{array}$ & $\mathrm{n} / \mathrm{a}$ & $\mathrm{n} / \mathrm{a}$ & $\mathrm{n} / \mathrm{a}$ & $\mathrm{n} / \mathrm{a}$ & $\mathrm{n} / \mathrm{a}$ & $\mathrm{n} / \mathrm{a}$ & $\mathrm{n} / \mathrm{a}$ & $\mathrm{n} / \mathrm{a}$ & $\mathrm{n} / \mathrm{a}$ & $\mathrm{n} / \mathrm{a}$ \\
\hline $\begin{array}{l}18 \\
19 \\
20 \\
21 \\
22\end{array}$ & $\begin{array}{c}\text { Germany }{ }^{\mathrm{B}} \\
\text { (EU-15, High GDP) }\end{array}$ & No & $\begin{array}{c}\text { Not } \\
\text { specified }\end{array}$ & No & GP, RHEUM & from day 3 & na & na & No & 78 weeks per 3 years & $\begin{array}{c}70 \% \text { of } \\
\text { brutto or } \\
90 \% \text { of } \\
\text { netto } \\
\text { income } \\
\end{array}$ & first 45 days & No \\
\hline $\begin{array}{l}23 \\
24 \\
25 \\
26\end{array}$ & $\begin{array}{c}\text { Greece }^{\mathrm{M}} \\
\text { (EU-15, Low GDP) }\end{array}$ & No & $\begin{array}{c}1-6 \\
\text { months }\end{array}$ & Yes & GP, RHEUM, physician ${ }^{6}$ & From day 1 & from day 1 & Day 16 & No & $\begin{array}{l}\text { maximum of sick- } \\
\text { leave in months equal } \\
\text { to the sum of years in } \\
\text { work }\end{array}$ & $\begin{array}{c}\text { not } \\
\text { possible to } \\
\text { define } \% \\
\end{array}$ & day $1-25$ & Yes \\
\hline $\begin{array}{l}27 \\
28 \\
29 \\
30\end{array}$ & $\begin{array}{c}\text { Hungary }{ }^{P} \\
\text { (EU-28, Low GDP) }\end{array}$ & No & $\begin{array}{c}\text { Not } \\
\text { specified }\end{array}$ & No & $\begin{array}{c}\text { GP, RHEUM, Supervisors } \\
\text { of the National Health } \\
\text { Insurance Fund } \\
\text { Administration }\end{array}$ & from day 1 & na & from day 30 & No & 370 days & $60 \%$ & day $1-380$ & Yes \\
\hline $\begin{array}{l}30 \\
31 \\
32\end{array}$ & $\begin{array}{c}\text { Iceland }^{\mathrm{S}} \\
\text { (Non-EU, High GDP) }\end{array}$ & No & $\begin{array}{l}\text { Depends } \\
\text { on work } \\
\text { union }\end{array}$ & No & GP, RHEUM & from day 1 & na & na & No & 48 months & $80-100 \%$ & $\begin{array}{l}\text { first } 3 \\
\text { months }\end{array}$ & No \\
\hline $\begin{array}{l}35 \\
34 \\
35\end{array}$ & $\begin{array}{c}\text { Ireland }{ }^{\mathrm{A}} \\
\text { (EU-15, High GDP) }\end{array}$ & Yes $^{2}$ & $\begin{array}{c}>=6 \\
\text { months }\end{array}$ & Yes & GP, RHEUM, Physician ${ }^{6}$ & from day 3 & from day 3 & na & No & 24 months & $\begin{array}{c}\text { not } \\
\text { possible to } \\
\text { define } \%^{8}\end{array}$ & $\begin{array}{c}\text { day } 7 \text { - } 1 \\
\text { year }\end{array}$ & Yes \\
\hline $\begin{array}{l}36 \\
37 \\
30\end{array}$ & $\begin{array}{c}\text { Israel }^{\mathrm{B}} \\
\text { (Non-EU, High GDP) }\end{array}$ & No & $\begin{array}{c}1-6 \\
\text { months }\end{array}$ & No & GP, RHEUM & from day 1 & na & na & Yes, 1 day & 3 months & $98 \%$ & first 90 days & Yes \\
\hline $\begin{array}{l}38 \\
39\end{array}$ & $\begin{array}{c}\text { Italy }^{\mathrm{M}} \\
\text { (EU-15, High GDP) }\end{array}$ & No & $\begin{array}{c}\text { Not } \\
\text { specified }\end{array}$ & No & GP & na & na & na & Yes, 3 days & 6 months & $64 \%$ & first 90 days & No \\
\hline
\end{tabular}




\begin{tabular}{|c|c|c|c|c|c|c|c|c|c|c|c|c|c|}
\hline $\begin{array}{l}1 \\
2\end{array}$ & $\begin{array}{c}\text { Latvia }^{\mathrm{P}} \\
\text { (EU-28, Low GDP) }\end{array}$ & No & $\begin{array}{c}\text { Not } \\
\text { specified }\end{array}$ & No & $\begin{array}{c}\text { GP, The Expert Medical } \\
\text { Commission }\end{array}$ & na & na & $\begin{array}{l}\text { from week } \\
26\end{array}$ & Yes, 1 day & $\begin{array}{c}26 \text { weeks, or } 52 \\
\text { weeks of the three } \\
\text { year period }\end{array}$ & $74 \%$ & day $2-10$ & No \\
\hline $\begin{array}{l}3 \\
4 \\
5 \\
6\end{array}$ & $\begin{array}{c}\text { Lithuania }^{\mathrm{P}} \\
\text { (EU-28, Low GDP) }\end{array}$ & No & $\begin{array}{c}1-6 \\
\text { months }\end{array}$ & No & $\begin{array}{l}\text { GP, RHEUM, physician } \\
\text { working at institution } \\
\text { which has contact with } \\
\text { Social Insurance Fund }\end{array}$ & From day 1 & na & na & No & $\begin{array}{c}\text { Continuous sick leave } \\
\text { of } 122 \text { days or Total } \\
\text { sick leave of } 153 \text { days } \\
\text { per year }\end{array}$ & $77 \%$ & first 2 days & No \\
\hline $\begin{array}{l}7 \\
8 \\
9 \\
10\end{array}$ & $\begin{array}{c}\text { Luxemburg }^{B} \\
\text { (EU-15, High GDP) }\end{array}$ & No & $\begin{array}{c}\text { Not } \\
\text { specified }\end{array}$ & No & $\begin{array}{l}\text { GP, RHEUM, any MD } \\
\text { approved by ministry of } \\
\text { health and not working } \\
\text { for social security }\end{array}$ & from day 1 & na & na & No & 12 months & $100 \%$ & first 90 days & No \\
\hline $\begin{array}{l}11 \\
12 \\
13\end{array}$ & $\begin{array}{l}\text { FYR Macedonia } \\
\text { (Non-EU, Low) }\end{array}$ & No & $\begin{array}{c}\text { Not } \\
\text { specified }\end{array}$ & No & $\begin{array}{c}\text { GP, RHEUM, Team } \\
\text { (Commission) of Three } \\
\text { Rheumatologists }\end{array}$ & from day 21 & na & from day 60 & No & 9 months & $87 \%$ & first 30 days & No \\
\hline $\begin{array}{l}14 \\
15 \\
16\end{array}$ & $\begin{array}{c}\text { Malta }^{\mathrm{M}} \\
\text { (EU-28, High GDP) }\end{array}$ & No & $\begin{array}{c}\text { Not } \\
\text { specified }\end{array}$ & No & $\begin{array}{c}\text { GP, RHEUM, any } \\
\text { physician working in } \\
\text { public sector }\end{array}$ & from day 1 & na & na & No & 156 days per year & $50 \%$ & first 3 days & No \\
\hline $\begin{array}{l}17 \\
18 \\
19 \\
20\end{array}$ & $\begin{array}{c}\text { Moldova } \\
\text { (Non-EU, Low GDP) }\end{array}$ & No & $\begin{array}{c}>=6 \\
\text { months }\end{array}$ & No & $\begin{array}{c}\text { GP, Medical Expertise } \\
\text { Committee (GP, RHEUM, } \\
\text { head of medical unit) }\end{array}$ & na & na & from day 7 & No & $\begin{array}{l}\text { Total sick leave of } \\
\text { calendar } 210 \text { days } \\
\text { (e.g. several periods } \\
\text { of sick leave) per }\end{array}$ & $60-90 \%$ & first 5 days & No \\
\hline $\begin{array}{l}21 \\
22\end{array}$ & $\begin{array}{c}\text { Montenegro }^{\mathrm{P}} \\
\text { (Non-EU, Low GDP) }\end{array}$ & No & $\begin{array}{c}\text { Not } \\
\text { specified }\end{array}$ & No & $\begin{array}{l}\text { GP, RHEUM, sick leave } \\
\text { grant commissions }\end{array}$ & from day 1 & na & from day 31 & No & 10 months & $70 \%$ & first 60 days & No \\
\hline 23 & $\begin{array}{c}\text { Norways } \\
\text { (Non-EU, High GDP) }\end{array}$ & No & $\begin{array}{c}1-6 \\
\text { months }\end{array}$ & No & $\begin{array}{l}\text { GP, RHEUM, physician } \\
\text { (working for employer) }\end{array}$ & from day 1 & from day 1 & na & No & 12 months & $100 \%$ & first 16 days & No \\
\hline $\begin{array}{l}25 \\
26 \\
27\end{array}$ & $\begin{array}{c}\text { PoLand }{ }^{\mathrm{P}} \\
\text { (EU-28, Low GDP) }\end{array}$ & No & $\begin{array}{c}1-6 \\
\text { months }\end{array}$ & No & $\begin{array}{c}\text { Any MD authorized by } \\
\text { the Social Insurance } \\
\text { Institution }\end{array}$ & from day 1 & na & na & No & $\begin{array}{l}182 \text { days ( } 6 \text { months) } \\
\text { per } 1 \text { year }\end{array}$ & $80 \%$ & first 30 days & No \\
\hline $\begin{array}{l}28 \\
29 \\
30\end{array}$ & $\begin{array}{c}\text { Portugal }{ }^{\mathrm{M}} \\
\text { (EU-15, High GDP) }\end{array}$ & No & $\begin{array}{c}1-6 \\
\text { months }\end{array}$ & No & $\begin{array}{c}\text { GP, RHEUM, any } \\
\text { physician working in } \\
\text { public sector }\end{array}$ & from day 1 & na & na & Yes, 3 days & $\begin{array}{c}1095 \text { days / } 36 \\
\text { months }\end{array}$ & $56 \%$ & $\begin{array}{c}\text { Emploer } \\
\text { does not } \\
\text { contribute }\end{array}$ & na \\
\hline 31 & $\begin{array}{c}\text { Romania }^{\mathrm{P}} \\
\text { (EU-28, Low GDP) }\end{array}$ & No & $\begin{array}{c}1-6 \\
\text { months }\end{array}$ & No & GP, RHEUM, physician ${ }^{6}$ & from day 1 & from day 1 & na & No & 6 months in one year & $75 \%$ & first 5 days & No \\
\hline 33 & $\begin{array}{c}\text { Russia }^{\mathrm{P}} \\
\text { (Non-EU,_Low GDP) }\end{array}$ & No & $\begin{array}{c}\text { Not } \\
\text { specified }\end{array}$ & No & GP, RHEUM & from day 1 & na & na & No & 12 months & $60-100 \%^{7}$ & first 3 days & na \\
\hline $\begin{array}{l}34 \\
35 \\
36 \\
37\end{array}$ & $\begin{array}{c}\text { Serbia }{ }^{P} \\
\text { (Non-EU, Low GDP) }\end{array}$ & No & $\begin{array}{c}\text { Not } \\
\text { specified }\end{array}$ & No & $\begin{array}{c}\text { GP, Board (Consilium of } 3 \\
\text { physicians) }\end{array}$ & na & na & $\begin{array}{l}\text { data not } \\
\text { provided }\end{array}$ & No & $\begin{array}{c}\text { There is no maximum } \\
\text { duration of sick leave } \\
\text { specified }\end{array}$ & $70-100 \%$ & first 4 weeks & No \\
\hline 38 & $\begin{array}{c}\text { Slovakia }^{\mathrm{P}} \\
\text { (EU-28, Low GDP) }\end{array}$ & No & $\begin{array}{c}1-6 \\
\text { months }\end{array}$ & Yes & $\begin{array}{c}\text { GP, Physician specialized } \\
\text { in occupational issues }\end{array}$ & na & week 52 & na & No & 12 months & $54 \%$ & first 10 days & no \\
\hline
\end{tabular}




\begin{tabular}{|c|c|c|c|c|c|c|c|c|c|c|c|c|c|}
\hline 1 & $\begin{array}{c}\text { Slovenia }{ }^{\mathrm{P}} \\
\text { (EU-28, High GDP) }\end{array}$ & No & $\begin{array}{c}\text { Not } \\
\text { specified }\end{array}$ & No & GP, Physician 5 & na & $\begin{array}{c}\text { from day } \\
30\end{array}$ & na & No & 12 months & $80 \%$ & first 30 days & No \\
\hline 3 & $\begin{array}{c}\text { Spain }^{\mathrm{M}} \\
\text { (EU-15, High GDP) }\end{array}$ & No & $\begin{array}{c}1-6 \\
\text { months }\end{array}$ & No & GP, rheumat. & From day 1 & na & na & Yes, 3 days & \begin{tabular}{|c|}
545 calendar days, or \\
18 months
\end{tabular} & $70 \%$ & day $4-15$ & No \\
\hline $\begin{array}{l}4 \\
5 \\
6\end{array}$ & $\begin{array}{c}\text { Sweden }^{\mathrm{S}} \\
\text { (EU-15, High GDP) }\end{array}$ & No & $\begin{array}{c}>=6 \\
\text { months }\end{array}$ & No & GP, RHEUM, Physician ${ }^{6}$ & from day 8 & \begin{tabular}{|c|} 
From day \\
8 \\
\end{tabular} & na & Yes, 1 day & 24 months & $79 \%$ & day $2-14$ & No \\
\hline $\begin{array}{l}0 \\
7 \\
8\end{array}$ & $\begin{array}{c}\text { Switzerland }{ }^{\mathrm{B}} \\
\text { (Non-EU, High GDP) }\end{array}$ & No & $\begin{array}{c}\text { Not } \\
\text { specified }\end{array}$ & No & GP, rheumat. & from day 1 & na & na & No & $\begin{array}{l}730 \text { days during a } \\
\text { period of } 900 \text { days }\end{array}$ & $\begin{array}{c}\text { not } \\
\text { possible to } \\
\text { define } \%\end{array}$ & first year & Yes \\
\hline $\begin{array}{l}9 \\
10 \\
11 \\
12 \\
13 \\
14\end{array}$ & $\begin{array}{c}\text { TaJikistan } \\
\text { (Non-EU, Low GDP) }\end{array}$ & Yes $^{1}$ & $\begin{array}{c}\text { Not } \\
\text { specified }\end{array}$ & No & $\begin{array}{l}\text { GP, RHEUM, the vice } \\
\text { director of the medical } \\
\text { institution together with } \\
\text { the family medical } \\
\text { doctor, rheumatologist } \\
\text { and/or main specialist }\end{array}$ & from day 7 & na & from day 7 & No & $\begin{array}{l}\text { Continuous sick leave } \\
\text { of } 120 \text { days or } \\
\text { interrupted sick leave } \\
\text { for } 150 \text { days during } \\
\text { past } 12 \text { month }\end{array}$ & $30-80 \%$ & first 14 days & No \\
\hline $\begin{array}{l}15 \\
16 \\
17\end{array}$ & $\begin{array}{l}\text { The Netherlands }{ }^{\mathrm{B}} \\
\text { (EU-15, High GDP) }\end{array}$ & No & $\begin{array}{c}\text { Not } \\
\text { specified }\end{array}$ & No & $\begin{array}{l}\text { Physician (working for } \\
\text { employer) specialized in } \\
\text { occupational issues }\end{array}$ & na & from day 1 & na & No & 24 months & $100 \%$ & first 2 years & No \\
\hline $\begin{array}{l}18 \\
19 \\
20\end{array}$ & $\begin{array}{c}\text { Turkey }^{\mathrm{M}} \\
\text { (Non-EU, Low GDP) }\end{array}$ & $\mathrm{No}^{3}$ & $\begin{array}{c}\text { Not } \\
\text { specified }\end{array}$ & No & $\begin{array}{l}\text { GP, RHEUM, physician }{ }^{6}, \\
\text { health commission }\end{array}$ & from day 1 & na & from day 40 & Yes, 3 days & 12 months & $63 \%$ & $\begin{array}{c}\text { Emploer } \\
\text { does not } \\
\text { contribute } \\
\end{array}$ & na \\
\hline $\begin{array}{l}21 \\
22 \\
23\end{array}$ & $\begin{array}{c}\text { Great Britain }{ }^{\mathrm{A}} \\
\text { (EU-15, High GDP) }\end{array}$ & No & $\begin{array}{c}\text { Not } \\
\text { specified }\end{array}$ & Yes & GP & na & na & na & No & $\begin{array}{c}28 \text { weeks (approx. } 7 \\
\text { months) }\end{array}$ & $\begin{array}{c}\text { not } \\
\text { possible to } \\
\text { define } \%^{8} \\
\end{array}$ & $\begin{array}{l}\text { first } 28 \\
\text { weeks }\end{array}$ & No \\
\hline $\begin{array}{l}24 \\
25 \\
26 \\
27\end{array}$ & $\begin{array}{c}\text { Ukraine }^{\mathrm{P}} \\
\text { (Non-EU, Low GDP) }\end{array}$ & No & $\begin{array}{c}\text { Not } \\
\text { specified }\end{array}$ & No & RHEUM, physician ${ }^{5}$ & from day 1 & $\begin{array}{l}\text { from day } \\
90\end{array}$ & na & No & $\begin{array}{c}\text { Continuous sick-leave } \\
\text { of } 3 \text { months or total } 4 \\
\text { months of sick-leave } \\
\text { during one year }\end{array}$ & $50-100 \%^{7}$ & $\begin{array}{c}\text { Emploer } \\
\text { does not } \\
\text { contribute }\end{array}$ & na \\
\hline
\end{tabular}

A - Anglo-saxon, S - Scandinavian, B - Bismarkian, M - Mediterranean, P - Post-communist

EU - European Union, GDP - Gross Domestic Product (classification based on GDP per capita in international dollars), MD - medical doctor, GP - general practitioner, RHEUM - rheumatologist

$\mathrm{Na}-$ not applicable

1 a person should be a citizen

2 Minimum 2 years

${ }^{3}$ but only citizen can get permanent contract)

${ }^{4}$ working for employer or public or private insurance institute and specialized in occupational issues

${ }^{5}$ working for public or private insurance company/institute

${ }^{6}$ working for public or private insurance company/institute or for employer

${ }^{7}$ depending on the work experience

8 fixed level of payment 
Online Supplementary Table S2. Social security system arrangements in case of work disability due to RA

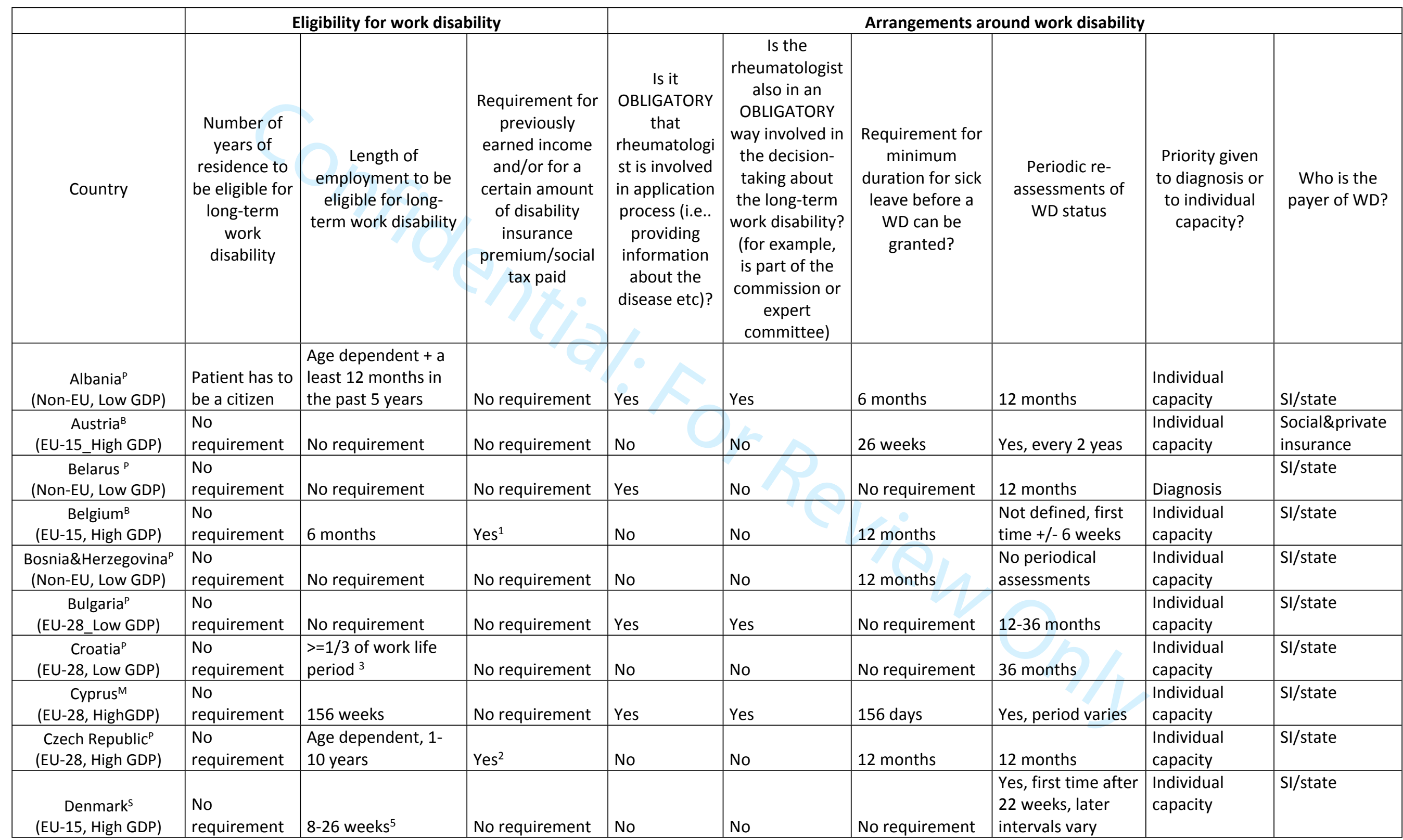




\begin{tabular}{|c|c|c|c|c|c|c|c|c|c|}
\hline $\begin{array}{c}\text { Eestonia }{ }^{\mathrm{P}} \\
\text { (EU-28, Low GDP) }\end{array}$ & \begin{tabular}{|l} 
No \\
requirement
\end{tabular} & $\begin{array}{l}\text { Age dependent, } 1 \text { to } \\
14 \text { years }\end{array}$ & No requirement & No & No & No requirement & 12 months & \begin{tabular}{|l} 
Individual \\
capacity
\end{tabular} & SI/state \\
\hline $\begin{array}{c}\text { Finland }^{\mathrm{s}} \\
\text { (EU-15, High GDP) }\end{array}$ & $\begin{array}{l}\text { No } \\
\text { requirement }\end{array}$ & No requirement & No & No & No & 12 months & \begin{tabular}{|l|} 
No periodical \\
assessments
\end{tabular} & \begin{tabular}{|l} 
Individual \\
capacity
\end{tabular} & $\begin{array}{l}\text { Social\&private } \\
\text { insurance }\end{array}$ \\
\hline $\begin{array}{c}\text { France }^{\mathrm{B}} \\
\text { (EU-15, High GDP) }\end{array}$ & $\begin{array}{l}\text { No } \\
\text { requirement }\end{array}$ & \begin{tabular}{|l|} 
No requirement for \\
disabled adult \\
allowance
\end{tabular} & Yes $^{6}$ & No & No & No requirement & 12 months & $\begin{array}{l}\text { Individual } \\
\text { capacity }\end{array}$ & SI/state \\
\hline $\begin{array}{c}\text { Georgia }^{\mathrm{P}} \\
\text { (Non-EU, Low GDP) }\end{array}$ & $n / a$ & $n / a$ & $n / a$ & $n / a$ & $n / a$ & $n / a$ & $n / a$ & $n / a$ & $n / a$ \\
\hline $\begin{array}{c}\text { Germany }^{\mathrm{B}} \\
\text { (EU-15, High GDP) }\end{array}$ & $\begin{array}{l}\text { No } \\
\text { requirement }\end{array}$ & No requirement & $Y_{e s}^{8}$ & No & No & No requirement & 3 years & \begin{tabular}{|l|} 
Individual \\
capacity
\end{tabular} & SI/state \\
\hline $\begin{array}{c}\text { Greece }^{\mathrm{M}} \\
\text { (EU-15, Low GDP) }\end{array}$ & $\begin{array}{l}\text { No } \\
\text { requirement }\end{array}$ & $\begin{array}{l}500 \text { days and last } \\
\text { day not longer than } \\
5 \text { years ago }\end{array}$ & No requirement & Yes & No & No requirement & $12-36$ months & $\begin{array}{l}\text { Individual } \\
\text { capacity }\end{array}$ & SI/state \\
\hline $\begin{array}{c}\text { Hungary }^{p} \\
\text { (EU-28, Low GDP) }\end{array}$ & $\begin{array}{l}\text { No } \\
\text { requirement }\end{array}$ & $\begin{array}{l}1095 \text { days in the } \\
\text { past } 5 \text { years }\end{array}$ & No requirement & No & No & No requirement & 3 years & \begin{tabular}{|l|} 
Individual \\
capacity
\end{tabular} & SI/state \\
\hline $\begin{array}{c}\text { Icelands } \\
\text { (Non-EU, High GDP) }\end{array}$ & 6 months & No requirement & No requirement & $\begin{array}{l}\text { data not } \\
\text { provided }\end{array}$ & $\begin{array}{l}\text { data not } \\
\text { provided }\end{array}$ & \begin{tabular}{|l|} 
varied depending \\
on age and \\
severity \\
\end{tabular} & $12-60$ months & $\begin{array}{l}\text { Individual } \\
\text { capacity }\end{array}$ & SI/state \\
\hline $\begin{array}{c}\text { Ireland } \\
\text { (EU-15, High GDP) }\end{array}$ & $\begin{array}{l}\text { No } \\
\text { requirement }\end{array}$ & 5 years & $\begin{array}{l}\text { earned income } \\
>40 \text { euro per } \\
\text { week }\end{array}$ & No & No & 12 months & $\begin{array}{l}\text { Yes, every } 6 \\
\text { months }\end{array}$ & $\begin{array}{l}\text { Diagnosis and } \\
\text { Individual } \\
\text { capacity }\end{array}$ & SI/state \\
\hline $\begin{array}{c}\text { Israel }^{\mathrm{B}} \\
\text { (Non-EU, High GDP) }\end{array}$ & $\begin{array}{l}\text { No } \\
\text { requirement }\end{array}$ & No requirement & No requirement & No & No & No requirement & $12-24$ months & $\begin{array}{l}\text { Individual } \\
\text { capacity }\end{array}$ & $\begin{array}{l}\text { Social\&private } \\
\text { insurance }\end{array}$ \\
\hline $\begin{array}{c}\text { Italy } \\
\text { (EU-15, High GDP) }\end{array}$ & $\begin{array}{l}\text { No } \\
\text { requirement }\end{array}$ & No requirement & No requirement & No & No & No requirement & Yes, period varies & $\begin{array}{l}\text { Individual } \\
\text { capacity }\end{array}$ & SI/state \\
\hline $\begin{array}{c}\text { Latvia }^{\mathrm{P}} \\
\left(\mathrm{EU}-28, \text { Low }^{\mathrm{a} D P}\right)\end{array}$ & $\begin{array}{l}\text { No } \\
\text { requirement }\end{array}$ & 36 months & No requirement & No & No & 26-52 weeks & $\begin{array}{l}\text { No periodical } \\
\text { assessments }\end{array}$ & Diagnosis & SI/state \\
\hline $\begin{array}{l}\text { Lithuania }^{\mathrm{P}} \\
\text { (EU-28, Low GDP) }\end{array}$ & Citizen & $\begin{array}{l}\text { Age dependent, 1- } \\
30 \text { years }\end{array}$ & No requirement & Yes & No & $\begin{array}{l}122 \text { (continuous) } \\
\text { or } 153 \text { (total) } \\
\text { days }\end{array}$ & $\begin{array}{l}\text { Yes, after } 6 \\
\text { months, } 1 \text { year or } \\
2 \text { years }\end{array}$ & $\begin{array}{l}\text { Individual } \\
\text { capacity }\end{array}$ & SI/state \\
\hline $\begin{array}{c}\text { Luxemburg B } \\
\text { (EU-15, High GDP) }\end{array}$ & $\begin{array}{l}\text { No } \\
\text { requirement }\end{array}$ & 6 months & No & No & No & 5 weeks & 12 months & \begin{tabular}{|l}
$\begin{array}{l}\text { Individual } \\
\text { capacity }\end{array}$ \\
\end{tabular} & SI/state \\
\hline $\begin{array}{l}\text { FYR Macedonia } \\
\text { (Non-EU, Low) }\end{array}$ & $\begin{array}{l}\text { No } \\
\text { requirement }\end{array}$ & 15 years & No requirement & Yes & No & 9 months & 24 months & \begin{tabular}{|l} 
Individual \\
capacity
\end{tabular} & SI/state \\
\hline $\begin{array}{c}\text { MaltaM } \\
\text { (EU-28, High GDP) }\end{array}$ & $\begin{array}{l}\text { No } \\
\text { requirement }\end{array}$ & 12 months & $\begin{array}{l}>=250 \\
\text { contributions to } \\
\text { social security }\end{array}$ & No & No & 6 months & 36 months & \begin{tabular}{|l} 
Individual \\
capacity
\end{tabular} & SI/state \\
\hline
\end{tabular}




\begin{tabular}{|c|c|c|c|c|c|c|c|c|c|}
\hline & & & $\begin{array}{l}\text { system and } \\
\text { annual average } \\
>=50 .\end{array}$ & & & & & & \\
\hline $\begin{array}{c}\text { Moldova }^{\mathrm{P}} \\
\text { (Non-EU, Low GDP) }\end{array}$ & Citizen & No requirement & No requirement & No & No & No requirement & 12 months & $\begin{array}{l}\text { Individual } \\
\text { capacity }\end{array}$ & SI/state \\
\hline $\begin{array}{c}\text { Montenegro }^{\mathrm{P}} \\
\text { (Non-EU, Low GDP) }\end{array}$ & $\begin{array}{l}\text { No } \\
\text { requirement }\end{array}$ & No requirement & No requirement & $\begin{array}{l}\text { data not } \\
\text { provided }\end{array}$ & $\begin{array}{l}\text { data not } \\
\text { provided }\end{array}$ & No requirement & $\begin{array}{l}\text { No periodical } \\
\text { assessments }\end{array}$ & $\begin{array}{l}\text { Individual } \\
\text { capacity }\end{array}$ & SI/state \\
\hline $\begin{array}{c}\text { Norways }^{\mathrm{s}} \\
\text { (Non-EU, High GDP) }\end{array}$ & 3 years & No requirement & No & No & No & 52 weeks & Yes, 2-3 years & $\begin{array}{l}\text { Individual } \\
\text { capacity }\end{array}$ & SI/state \\
\hline $\begin{array}{c}\text { PoLand } \\
\text { (EU-28, Low GDP) }\end{array}$ & $\begin{array}{l}\text { No } \\
\text { requirement }\end{array}$ & $\begin{array}{l}\text { Minimum } 5 \text { years } \\
\text { during last } 10 \text { years }\end{array}$ & No requirement & No & No & 182 days & 12 months & $\begin{array}{l}\text { Individual } \\
\text { capacity }\end{array}$ & SI/state \\
\hline $\begin{array}{c}\text { Portugal }^{\mathrm{M}} \\
\text { (EU-15, High GDP) }\end{array}$ & $\begin{array}{l}\text { No } \\
\text { requirement }\end{array}$ & $\begin{array}{l}120 \text { worked days in } \\
\text { last } 3-5 \text { years }\end{array}$ & No requirement & No & No & No requirement & 36 months & $\begin{array}{l}\text { Individual } \\
\text { capacity }\end{array}$ & SI/state \\
\hline $\begin{array}{c}\text { Romania }^{\mathrm{P}} \\
(\mathrm{EU}-28, \text { Low GDP) }\end{array}$ & Citizen & $\begin{array}{l}\text { Age dependent, } 1 \text { - } \\
27 \text { years }\end{array}$ & No requirement & No & No & 183 days & $12-36$ months & $\begin{array}{l}\text { Individual } \\
\text { capacity }\end{array}$ & SI/state \\
\hline $\begin{array}{c}\text { RussiaP }^{\mathrm{P}} \\
\text { (Non-EU,_Low GDP) }\end{array}$ & $\begin{array}{l}\text { No } \\
\text { requirement }\end{array}$ & No requirement & No requirement & Yes & No & No requirement & $1-2$ years & $\begin{array}{l}\text { Individual } \\
\text { capacity }\end{array}$ & SI/state \\
\hline $\begin{array}{c}\text { Serbia }{ }^{\mathrm{P}} \\
\text { (Non-EU, Low GDP) }\end{array}$ & $\begin{array}{l}\text { No } \\
\text { requirement }\end{array}$ & No requirement & No requirement & No & No & 6 months & $\begin{array}{l}\text { No periodical } \\
\text { assessments }\end{array}$ & $\begin{array}{l}\text { Individual } \\
\text { capacity }\end{array}$ & SI/state \\
\hline $\begin{array}{c}\text { Slovakia }^{\mathrm{P}} \\
\text { (EU-28, Low GDP) }\end{array}$ & $\begin{array}{l}\text { No } \\
\text { requirement }\end{array}$ & $\begin{array}{l}\text { Age dependent, } 1 \text { - } \\
15 \text { years }\end{array}$ & No requirement & Yes & Yes & 12 months & Yes, period varies & $\begin{array}{l}\text { Individual } \\
\text { capacity }\end{array}$ & SI/state \\
\hline $\begin{array}{c}\text { Slovenia }{ }^{\mathrm{P}} \\
\text { (EU-28, High GDP) }\end{array}$ & $\begin{array}{l}\text { No } \\
\text { requirement }\end{array}$ & $\begin{array}{l}\text { Age dependent, >= } \\
1 / 3 \text { of the period } \\
\text { 'age of disability - } \\
20 \text { ' }\end{array}$ & No requirement & No & No & No requirement & Yes, $12-60$ months & $\begin{array}{l}\text { Individual } \\
\text { capacity }\end{array}$ & SI/state \\
\hline $\begin{array}{c}\text { Spain }^{M} \\
\text { (EU-15, High GDP) }\end{array}$ & $\begin{array}{l}\text { No } \\
\text { requirement }\end{array}$ & $\begin{array}{l}\text { Min } 1800 \text { working } \\
\text { days in preceding } 10 \\
\text { years for partial WD }\end{array}$ & No requirement & No & No & No requirement & 2 years & $\begin{array}{l}\text { Individual } \\
\text { capacity }\end{array}$ & SI/state \\
\hline $\begin{array}{c}\text { Switzerland }^{\mathrm{B}} \\
\text { (Non-EU, High GDP) }\end{array}$ & \begin{tabular}{|l|} 
No \\
requirement
\end{tabular} & No requirement & No requirement & No & No & 2 years & 5-10 years & $\begin{array}{l}\text { Individual } \\
\text { capacity }\end{array}$ & $\begin{array}{l}\text { Social\&private } \\
\text { insurance }\end{array}$ \\
\hline
\end{tabular}




\section{Annals of the Rheumatic Diseases}

\begin{tabular}{|c|c|c|c|c|c|c|c|c|c|}
\hline $\begin{array}{c}\text { TaJikistan } \\
\text { (Non-EU, Low GDP) }\end{array}$ & Citizen & No requirement & No requirement & Yes & Yes & 120 working days & $\begin{array}{l}\text { Yes, every } 11 \\
\text { months }\end{array}$ & \begin{tabular}{|l} 
Individual \\
capacity
\end{tabular} & $\mathrm{SI} /$ state \\
\hline $\begin{array}{l}\text { The Netherlands }{ }^{\mathrm{B}} \\
\text { (EU-15, High GDP) }\end{array}$ & $\begin{array}{l}\text { No } \\
\text { requirement }\end{array}$ & No requirement & No requirement & No & No & 24 months & $\begin{array}{l}\text { Yes, period varies } \\
\text { and depends on } \\
\text { diagnosis } \\
\end{array}$ & \begin{tabular}{|l} 
Individual \\
capacity
\end{tabular} & $\mathrm{SI} /$ state \\
\hline $\begin{array}{c}\text { TurkeyM }^{M} \\
\text { (Non-EU, Low GDP) }\end{array}$ & \begin{tabular}{|l} 
No \\
requirement
\end{tabular} & 120 months & No requirement & No & No & No requirement & 2 years & \begin{tabular}{|l|} 
Individual \\
capacity \\
\end{tabular} & $\mathrm{SI} /$ state \\
\hline $\begin{array}{c}\text { Great Britain } \\
\text { (EU-15, High GDP) }\end{array}$ & $\begin{array}{l}\begin{array}{l}\text { No } \\
\text { requirement }\end{array} \\
\end{array}$ & No requirement & Yes $^{4}$ & No & No & 28 weeks & $\begin{array}{l}\text { Yes, period varies } \\
\text { and depends on } \\
\text { diagnosis } \\
\end{array}$ & \begin{tabular}{|l|} 
Individual \\
capacity
\end{tabular} & SI/state \\
\hline $\begin{array}{c}\text { Ukraine } \\
\text { (Non-EU, Low GDP) }\end{array}$ & $\begin{array}{l}\text { No } \\
\text { requirement }\end{array}$ & No requirement & No requirement & No & No & 3 months & $\begin{array}{l}\text { Yes, every } 12 \\
\text { months }\end{array}$ & \begin{tabular}{|l} 
Individual \\
capacity
\end{tabular} & $\mathrm{SI} /$ state \\
\hline
\end{tabular}

A - Anglo-saxon, S - Scandinavian, B - Bismarkian, M - Mediterranean, P - Post-communist

EU - European Union, GDP - Gross Domestic Product (classification based on GDP per capita in international dollars) , SI - social insurance

1 1501,82 / 1082,65 euro per trimester for a person >=21 / <21 years old, resp

$2>=36$ months of payments to the statutory pension insurance within the last 5 years

${ }^{3}$ from 20-26 y.o. depending on education

${ }^{4}$ depends on multiple factors of individual situation (online calculator)

${ }^{5}$ Public benefit: continuous employment, the last 26 weeks (min. 240 hours) Employer-paid benefit: 8 weeks of employment (min. 74 hours)

${ }^{6}$ Previously earned income 19,142.9 euro for invalidity pension / 9,482.16 euro for disabled adult allowance

${ }^{7} 800$ working hours in previous 12 months for invalidity pension

$8>=36$ months of payments to the statutory pension insurance within the last 5 years 


\section{Online Supplementary Table S3. Regulations to prevent work disability and help staying at work for people with RA}

\begin{tabular}{|c|c|c|c|c|c|c|c|c|c|c|c|c|}
\hline \multirow[b]{2}{*}{ Country } & \multicolumn{8}{|c|}{ Facilities available to support stay at work } & \multirow[b]{2}{*}{\begin{tabular}{|} 
Participatio \\
$n$ in \\
programs \\
to facilitate \\
employmen \\
t obligatory \\
before a \\
WD can be \\
granted?
\end{tabular}} & \multicolumn{2}{|c|}{ Working with disability } & \multirow[b]{2}{*}{$\begin{array}{c}\text { Forms of societal support for employment } \\
\text { of people with disabilities }\end{array}$} \\
\hline & $\begin{array}{c}\text { Yes/N } \\
0\end{array}$ & $\begin{array}{c}\text { Vocati } \\
\text { onal } \\
\text { rehabi } \\
\text { litatio } \\
\mathrm{n} \text { in } \\
\text { the } \\
\text { hospit } \\
\text { al }\end{array}$ & $\begin{array}{c}\text { Vocatio } \\
\text { nal } \\
\text { rehabilit } \\
\text { ation in } \\
\text { private } \\
\text { sector }\end{array}$ & $\begin{array}{l}\text { Self- } \\
\text { learning } \\
\text { /self- } \\
\text { manage } \\
\text { ment }\end{array}$ & $\begin{array}{c}\text { Work } \\
\text { coaches }\end{array}$ & $\begin{array}{l}\text { Advice } \\
\text { from } \\
\text { occupati } \\
\text { onal } \\
\text { physicia } \\
n\end{array}$ & $\begin{array}{l}\text { Coaching } \\
\text { (advice) } \\
\text { from } \\
\text { employm } \\
\text { ent } \\
\text { agency }\end{array}$ & Other & & $\begin{array}{c}\text { Can a } \\
\text { person } \\
\text { with WD } \\
\text { perform a } \\
\text { paid } \\
\text { work? }\end{array}$ & $\begin{array}{l}\text { Does the } \\
\text { right to } \\
\text { work } \\
\text { depend on } \\
\text { degree of } \\
\text { disability? }\end{array}$ & \\
\hline $\begin{array}{c}\text { Albania }^{\mathrm{P}} \\
\text { (Non-EU, Low GDP) }\end{array}$ & Yes & Yes & No & Yes & No & No & No & & No & Yes & Yes & reductions in taxes for employers \\
\hline $\begin{array}{c}\text { Austria }^{\mathrm{B}} \\
\text { (EU-15_High GDP) }\end{array}$ & Yes & No & Yes & No & No & Yes & No & $\otimes$ & Yes & Yes $^{6}$ & No & $\begin{array}{l}\text { protection against dismissal; wage protection; } \\
\text { additional annual leave; benefits for the } \\
\text { employer and his business if disabled people } \\
\text { are employed; tax benefits }\end{array}$ \\
\hline $\begin{array}{c}\text { Belarus }^{\mathrm{P}} \\
\text { (Non-EU, Low GDP) }\end{array}$ & Yes & Yes & No & Yes & No & No & No & & No & Yes & Yes & reductions in taxes for employers \\
\hline $\begin{array}{c}\text { Belgium }^{\mathrm{B}} \\
\text { (EU-15, High GDP) }\end{array}$ & Yes & Yes & No & No & Yes & Yes & Yes & Yes $^{1}$ & No & Yes & Yes & $\begin{array}{l}\text { compensation for work place adjustments, } \\
\text { travel expenses, subsidies for employers }\end{array}$ \\
\hline $\begin{array}{l}\text { Bosnia\&Herzegovina }{ }^{P} \\
\text { (Non-EU, Low GDP) }\end{array}$ & No & na & na & na & na & na & na & & No & No & NA & none \\
\hline $\begin{array}{c}\text { Bulgaria }^{\mathrm{P}} \\
\text { (EU-28_Low GDP) }\end{array}$ & Yes & Yes & No & Yes & No & Yes & Yes & & No & Yes & No & $\begin{array}{l}\text { obligatory quotas of patients with disabilities } \\
\text { that should be employed. }\end{array}$ \\
\hline $\begin{array}{c}\text { Croatia }^{\mathrm{P}} \\
\text { (EU-28, Low GDP) }\end{array}$ & Yes & No & No & No & No & Yes & Yes & Yes $^{2}$ & No & Yes $^{7}$ & No & $\begin{array}{l}\text { obligatory quotas of patients with disabilities } \\
\text { that should be employed. }\end{array}$ \\
\hline $\begin{array}{c}\text { Cyprus }^{\mathrm{M}} \\
\text { (EU-28, HighGDP) }\end{array}$ & Yes & Yes & Yes & Yes & Yes & Yes & Yes & & No & Yes & Yes & $\begin{array}{l}\text { obligatory quotas of patients with disabilities } \\
\text { that should be employed, subsidies for } \\
\text { companies, programs of additional education } \\
\text { and training }\end{array}$ \\
\hline $\begin{array}{l}\text { Czech Republic }^{P} \\
\text { (EU-28, High GDP) }\end{array}$ & Yes & Yes & Yes & Yes & No & Yes & No & & No & Yes & No & reductions in taxes for employers \\
\hline $\begin{array}{c}\text { Denmark }^{\mathrm{S}} \\
\text { (EU-15, High GDP) }\end{array}$ & Yes & Yes & Yes & Yes & Yes & Yes & Yes & & Yes & Yes & Yes & wages subsidies \\
\hline $\begin{array}{c}\text { Eestonia }^{\mathrm{P}} \\
\text { (EU-28, Low GDP) }\end{array}$ & No & na & na & na & na & na & na & & No & Yes & No & reductions in taxes for employers \\
\hline $\begin{array}{c}\text { Finlands }^{\mathrm{s}} \\
\text { (EU-15, High GDP) }\end{array}$ & Yes & Yes & Yes & Yes & No & Yes & Yes & & Yes & Yes & Yes & $\begin{array}{l}\text { subsidies for companies, programs of } \\
\text { additional education and training }\end{array}$ \\
\hline
\end{tabular}




\begin{tabular}{|c|c|c|c|c|c|c|c|c|c|c|c|c|}
\hline $\begin{array}{c}\text { France }^{\mathrm{B}} \\
\text { (EU-15, High GDP) }\end{array}$ & No & na & na & na & na & na & na & & No & Yes & No & $\begin{array}{l}\text { obligatory quotas of patients with disabilities } \\
\text { that should be employed. }\end{array}$ \\
\hline $\begin{array}{c}\text { Georgia }^{P} \\
\text { (Non-EU, Low GDP) }\end{array}$ & No & na & na & na & na & na & na & & $n / a$ & na & na & none \\
\hline $\begin{array}{c}\text { Germany }^{B} \\
\text { (EU-15, High GDP) }\end{array}$ & Yes & No & Yes & Yes & Yes & Yes & Yes & & No & Yes $^{6}$ & Yes & $\begin{array}{l}\text { obligatory quotas of patients with disabilities } \\
\text { that should be employed. }\end{array}$ \\
\hline $\begin{array}{c}\text { Greece }^{\mathrm{M}} \\
\text { (EU-15, Low GDP) }\end{array}$ & No & na & na & na & na & na & na & & No & Yes $^{7}$ & Yes & none \\
\hline $\begin{array}{c}\text { Hungary }^{p} \\
\text { (EU-28, Low GDP) }\end{array}$ & No & na & na & na & na & na & na & & No & Yes & Yes & $\begin{array}{l}\text { obligatory quotas of patients with disabilities } \\
\text { that should be employed. }\end{array}$ \\
\hline $\begin{array}{c}\text { Iceland }^{\mathrm{S}} \\
\text { (Non-EU, High GDP) }\end{array}$ & Yes & Yes & Yes & & & Yes & & & No & Yes & No & subsidies for companies \\
\hline $\begin{array}{c}\text { Ireland }{ }^{\mathrm{A}} \\
\text { (EU-15, High GDP) }\end{array}$ & Yes & No & Yes & Yes & No & Yes & No & & No & Yes & Yes & $\begin{array}{l}\text { obligatory quotas of patients with disabilities } \\
\text { that should be employed, subsidies for } \\
\text { companies, workplace adaptations }\end{array}$ \\
\hline $\begin{array}{c}\text { Israel }^{\mathrm{B}} \\
\text { (Non-EU, High GDP) }\end{array}$ & Yes & Yes & Yes & No & No & Yes & No & & No & Yes & No & education programs \\
\hline $\begin{array}{c}\text { ItalyM } \\
\text { (EU-15, High GDP) }\end{array}$ & Yes & No & No & Yes & No & No & No & & No & Yes & Yes & $\begin{array}{l}\text { obligatory quotas of patients with disabilities } \\
\text { that should be employedYes }\end{array}$ \\
\hline $\begin{array}{c}\text { Latvia }^{\mathrm{P}} \\
\text { (EU-28, Low GDP) }\end{array}$ & No & na & na & na & na & na & na & & No & Yes & No & subsidies for companies \\
\hline $\begin{array}{c}\text { Lithuania }^{\mathrm{P}} \\
\text { (EU-28, Low GDP) }\end{array}$ & Yes & Yes & Yes & Yes & & Yes & Yes & 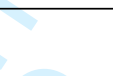 & Yes & Yes & Yes & Education programs \\
\hline $\begin{array}{c}\text { Luxemburg } \\
\text { (EU-15, High GDP) }\end{array}$ & Yes & No & No & No & No & Yes & No & Yes $^{2}$ & No & Yes & No & yes, details not specified \\
\hline $\begin{array}{l}\text { FYR Macedonia } \\
\text { (Non-EU, Low) }\end{array}$ & Yes & Yes & Yes & Yes & No & Yes & No & & No & No & NA & $\begin{array}{l}\text { reductions in taxes for employers (1 year of } \\
\text { employment) }\end{array}$ \\
\hline $\begin{array}{c}\text { MaltaM } \\
\text { (EU-28, High GDP) }\end{array}$ & No & na & na & na & na & na & na & & No & Yes & No & $\begin{array}{l}\text { reductions in taxes for employers, subsidies } \\
\text { for companies, obligatory quotas of patients } \\
\text { with disabilities that should be employed }\end{array}$ \\
\hline $\begin{array}{c}\text { Moldova }{ }^{\mathrm{P}} \\
\text { (Non-EU, Low GDP) }\end{array}$ & Yes & Yes & Yes & Yes & No & No & Yes & & Yes & Yes & Yes & $\begin{array}{l}\text { obligatory quotas of patients with disabilities } \\
\text { that should be employed }\end{array}$ \\
\hline $\begin{array}{c}\text { Montenegro } \\
\text { (Non-EU, Low GDP) }\end{array}$ & Yes & Yes & No & No & No & No & No & & Yes & Yes & Yes & yes, details not specified \\
\hline $\begin{array}{c}\text { Norways } \\
\text { (Non-EU, High GDP) }\end{array}$ & Yes & yes & No & No & No & Yes & yes & & Yes & $\begin{array}{l}\text { Yes }(\text { after } \\
1 \text { year) }\end{array}$ & No & $\begin{array}{l}\text { in case of re-entering employment, employer } \\
\text { may for the first months (up to one year) get } \\
\text { refund of salary by the social security system. }\end{array}$ \\
\hline $\begin{array}{c}\text { PoLand } \\
\text { (EU-28, Low GDP) }\end{array}$ & Yes & Yes & No & Yes & Yes & Yes & Yes & Yes $^{4}$ & No & Yes & Yes & $\begin{array}{l}\text { obligatory quotas of patients with disabilities } \\
\text { that should be employed, educational } \\
\text { programs, subsidies for companies }\end{array}$ \\
\hline Romania $^{P}$ & Yes & No & No & No & No & Yes & No & & Yes & Yes & Yes & None \\
\hline
\end{tabular}




\begin{tabular}{|c|c|c|c|c|c|c|c|c|c|c|c|c|}
\hline (EU-28, Low GDP) & & & & & & & & & & & & \\
\hline $\begin{array}{c}\text { RussiaP }^{\mathrm{P}} \\
\text { (Non-EU,_Low GDP) }\end{array}$ & No & na & na & na & na & na & na & & No & No & NA & $\begin{array}{l}\text { "supported employment" }{ }^{\text {, }} \text { obligatory quotas } \\
\text { of patients with disabilities that should be } \\
\text { employed }\end{array}$ \\
\hline $\begin{array}{c}\text { Serbia } \\
\text { (Non-EU, Low GDP) }\end{array}$ & Yes & Yes & No & Yes & No & No & No & & No & No & NA & yes, details not specified \\
\hline $\begin{array}{c}\text { Slovakiap }^{\mathrm{P}} \\
\text { (EU-28, Low GDP) }\end{array}$ & Yes & Yes & Yes & No & No & Yes & No & & No & Yes & No & $\begin{array}{l}\text { obligatory quotas of patients with disabilities } \\
\text { that should be employed }\end{array}$ \\
\hline $\begin{array}{c}\text { Slovenia }^{P} \\
\text { (EU-28, High GDP) }\end{array}$ & Yes & No & No & Yes & No & Yes & Yes & & Yes & Yes $^{6}$ & Yes & $\begin{array}{l}\text { obligatory quotas of patients with disabilities } \\
\text { that should be employed }\end{array}$ \\
\hline $\begin{array}{c}\text { Spain }^{M} \\
\text { (EU-15, High GDP) }\end{array}$ & No & na & na & na & na & na & na & & No & Yes & Yes & $\begin{array}{l}\text { subsidies for companies, reductions in taxes } \\
\text { for employers }\end{array}$ \\
\hline $\begin{array}{c}\text { Sweden }^{\mathrm{s}} \\
\text { (EU-15, High GDP) }\end{array}$ & Yes & No & yes & Yes & No & Yes & Yes & & Yes & Yes & Yes & $\begin{array}{l}\text { subsidies for companies, reductions in taxes } \\
\text { for employers }\end{array}$ \\
\hline $\begin{array}{c}\text { Switzerland } \\
\text { (Non-EU, High GDP) }\end{array}$ & Yes & Yes & No & No & No & Yes & No & & Yes & Yes $^{6}$ & Yes & $\begin{array}{l}\text { "supported employment", "sheltered } \\
\text { employment", or social enterprises with } \\
\text { partially subsidized job } \\
\end{array}$ \\
\hline $\begin{array}{c}\text { TaJikistan } \\
\text { (Non-EU, Low GD) }\end{array}$ & Yes & Yes & No & Yes & Yes & Yes & No & & No & Yes & No & $\begin{array}{l}\text { reductions in taxes for employers, educational } \\
\text { programs }\end{array}$ \\
\hline $\begin{array}{c}\text { Turkey }^{\mathrm{M}} \\
\text { (Non-EU, Low GDP) }\end{array}$ & No & na & na & na & na & na & na & & No & Yes & Yes & $\begin{array}{l}\text { obligatory quotas of patients with disabilities } \\
\text { that should be employed }\end{array}$ \\
\hline $\begin{array}{c}\text { Great Britain }{ }^{\mathrm{A}} \\
(\mathrm{EU}-15, \text { High GDP) }\end{array}$ & Yes & No & No & No & No & No & No & Yes $^{5}$ & No & Yes $^{6}$ & No & yes, details not specified \\
\hline $\begin{array}{c}\text { Ukraine }^{\mathrm{P}} \\
\text { (Non-EU, Low GDP) }\end{array}$ & No & na & na & na & na & na & na & & No & Yes & No & None \\
\hline
\end{tabular}

A - Anglo-saxon, S - Scandinapean Union, GDP - Gross Domestic Product (classification based on GDP per capita in international dollars)

1 Progressive work reintegration

2 Professional rehabilitation right provided and payed by social insurance

3 Internal or external reclassification coached by social security

${ }^{4}$ Working in protected conditions, conditions of employment in sheltered workshops, customized workplace

${ }^{5}$ An occupational health assessment with recommendations made to their employer and doctor

6 With a limitation on earnings

${ }^{7}$ Amount of pension may be reduced

8 Salaries may be lower than average disability pension 
Online Supplementary Table S4. Patients' and rheumatologists' characteristics.

\begin{tabular}{|c|c|}
\hline & Total \\
\hline \multicolumn{2}{|l|}{ Rheumatologists characteristics (Questionnaire 2) } \\
\hline $\mathrm{N}$ of rheumatologists ( $\mathrm{N}$ of countries) & $539(33)$ \\
\hline Age (years) & $47.5(10.2)$ \\
\hline Gender, female & $284(53 \%)$ \\
\hline \multicolumn{2}{|l|}{ Current work environment } \\
\hline Non-university hospital ${ }^{* *}$ & $142(26 \%)$ \\
\hline University hospital & $278(51 \%)$ \\
\hline Private practice & $52(10 \%)$ \\
\hline Other & $22(4 \%)$ \\
\hline Years of work experience & $2.5(1.1)$ \\
\hline Average hours of patient care per week & $33.5(12.3)$ \\
\hline \multicolumn{2}{|l|}{ Patient characteristics (Questionnaire 3) } \\
\hline $\mathrm{N}$ of patients ( $\mathrm{N}$ of countries) & $719(34)$ \\
\hline Age (years) & $53.1(12.2)$ \\
\hline Gender, female & $541(76 \%)$ \\
\hline \multicolumn{2}{|l|}{ Education } \\
\hline Primary & $110(16 \%)$ \\
\hline Secondary & $254(36 \%)$ \\
\hline High & $339(48 \%)$ \\
\hline \multicolumn{2}{|l|}{ Work status } \\
\hline Paid work & $317(45 \%)$ \\
\hline No paid work* & $180(26 \%)$ \\
\hline Work disabled (WD) & $172(24 \%)$ \\
\hline Paid work + WD & $34(5 \%)$ \\
\hline Symptom duration (years) & $15.0(11.1)$ \\
\hline Ever absent from work due to RA & $472(69 \%)$ \\
\hline
\end{tabular}

Values reflect mean (SD) or $\mathrm{n}(\%)$, as appropriate; WD - work disability * but not work disabled ** private or public Anglosaxon: UK, Ireland; Scandinavian: Denmark, Iceland, Sweden, Norway, Finland; Bismarkian: Austria, Belgium, Germany, France, Israel, Netherlands, Switzerland; Mediterranean: Cyprus, Greece, Italy, Portugal, Spain, Turkey; Postcommunist: Albania, Bulgaria, Czech Republic, Croatia, Estonia, Georgia, Hungary, Latvia, Lithuania, Poland, Romania, Russian Federation, Serbia, Tajikistan, Slovak Republic, Slovenia 
Online Supplementary Table S5. Patients' and rheumatologists' perceptions across several domains according to (1) GDP per capita PPP (2) EU membership status (3) Type of social security system

\begin{tabular}{|c|c|c|c|c|c|}
\hline & \multicolumn{3}{|c|}{ Patients' perceptions $\xi$} & \multicolumn{2}{|c|}{ Rheumatologists' perceptions $\xi$} \\
\hline & $\begin{array}{l}\text { Importance and } \\
\text { support to remain } \\
\text { employed }(0-5) \\
\mathrm{n}=520\end{array}$ & $\begin{array}{l}\text { Process of } \\
\text { applying for work } \\
\text { disability pension } \\
(0-4) \\
n=360\end{array}$ & $\begin{array}{l}\text { Obtaining and } \\
\text { living with work } \\
\text { disability pension } \\
(0-6) \\
n=358\end{array}$ & $\begin{array}{l}\text { Performance of the } \\
\text { system }(0-4) \\
\mathrm{n}=498\end{array}$ & $\begin{array}{l}\text { Role of the } \\
\text { rheumatologists } \\
(0-4) \\
n=507\end{array}$ \\
\hline $\begin{array}{l}\text { GDP per capita (int. \$) } \\
\qquad \text { High vs low GDP }\end{array}$ & $0.16[-0.12 ; 0.44]$ & $0.36[-0.27 ; 0.99]$ & $-0.13[-0.49 ; 0.23]$ & $0.31[-0.08 ; 0.69]$ & $-0.54[-0.89 ;-0.20]$ \\
\hline $\begin{array}{l}\text { EU membership } \\
\text { EU vs non-EU member }\end{array}$ & $0.19[-0.13 ; 0.52]$ & $-0.43[-1.17 ; 0.32]$ & $-0.19[-0.61 ; 0.24]$ & $0.02[-0.43 ; 0.46]$ & $-0.42[-0.83 ;-0.00]$ \\
\hline $\begin{array}{r}\text { Type of system } \\
\text { Scandinavian } \\
\text { Anglo-Saxon/liberal } \\
\text { Bismarckian/conservative } \\
\text { Mediterranean/southern } \\
\text { Post-Communist/eastern }\end{array}$ & $\begin{array}{c}\text { Reference } \\
-1.02[-1.62 ;-0.42] \\
-0.39[-0.88 ; 0.11] \\
-0.55[-1.04 ;-0.06] \\
-0.58[-1.00 ;-0.15]\end{array}$ & $\begin{array}{c}\text { Reference } \\
-1.87[-3.24 ;-0.50] \\
-1.02[-2.15 ; 0.12] \\
-1.37[-2.46 ;-0.29] \\
-1.14[-2.07 ;-0.22]\end{array}$ & $\begin{array}{c}\text { Reference } \\
-0.42[-1.25 ; 0.41] \\
-0.02[-0.71 ; 0.67] \\
-0.25[-0.90 ; 0.41] \\
-0.11[-0.67 ; 0.46]\end{array}$ & $\begin{array}{c}\text { Reference } \\
-\mathbf{1 . 4 0}[-2.14 ;-0.67] \\
-0.29[-0.79 ; 0.21] \\
-1.18[-1.68 ;-0.67] \\
-0.86[-1.30 ;-0.42]\end{array}$ & $\begin{array}{c}\text { Reference } \\
-0.60[-1.47 ; 0.27] \\
-0.34[-0.95 ; 0.27] \\
-0.10[-0.71 ; 0.51] \\
0.24[-0.29 ; 0.77]\end{array}$ \\
\hline
\end{tabular}

Coefficients are derived from multilevel models (with individuals clustered in countries) and each independent variable (i.e. GDP, EU membership or type of system) entered in a separate model; GDP - Gross Domestic Product, EU - European Union

Anglo-saxon: UK, Ireland; Scandinavian: Denmark, Iceland, Sweden, Norway, Finland; Bismarkian: Austria, Belgium, Germany, France, Israel, Netherlands, Switzerland; Mediterranean: Cyprus, Greece, Italy, Portugal, Spain, Turkey; Post-communist: Albania, Bulgaria, Czech Republic, Croatia, Estonia, Georgia, Hungary, Latvia, Lithuania, Poland, Romania, Russian Federation, Serbia, Tajikistan, Slovak Republic, Slovenia

sthe higher score the more positive are the perception 
Online Supplementary Table S6. N(\%) of missing data per variable.

\begin{tabular}{|l|l|}
\hline & N (\%) missing \\
\hline Rheumatologists characteristics (Questionnaire 2) & \\
\hline N of rheumatologists (N of countries) & $539(33)$ \\
\hline Age (years) & $136(25.2)$ \\
\hline Gender, female & $43(8.0)$ \\
\hline Current work environment & $47(8.7)$ \\
\hline Years of work experience & $45(8.4)$ \\
\hline Average hours of patient care per week & $53(9.8)$ \\
\hline Role of the rheumatologists (0-4) & $32(5.9)$ \\
\hline Performance of the system (0-4) & $41(7.6)$ \\
\hline Patient characteristics (Questionnaire 3) & \\
\hline N of patients (N of countries) & $719(34)$ \\
\hline Age (years) & $18(2.5)$ \\
\hline Gender, female & $19(2.6)$ \\
\hline Education & $30(4.1)$ \\
\hline Work status & $30(4.1)$ \\
\hline Symptom duration (years) & $32(4.4)$ \\
\hline Ever absent from work due to RA & $46(6.3)$ \\
\hline Importance and support to remain employed (0-5) & $53(9.3)^{\star}$ \\
\hline Process of applying for WD (0-4) & $78(17.8)^{\star *}$ \\
\hline Obtaining and living with WD pension (0-6) & $80(19.2)^{\star *}$ \\
\hline
\end{tabular}

WD - work disability * but not work disabled ${ }^{* *}$ private or public

*only assessed in patients currently or ever having worked $(n=573)$

**only assessed in patients currently work disabled or ever considered applying for WD $(n=438)$ 


\section{Online supplementary material}

\section{Contents}

Online Supplementary Text S1. Questionnaire administered to Principal Investigators (one



Online Supplementary Text S2. Questionnaire for rheumatologists (Survey 2)................................23

Online Supplementary Text S3. Questionnaire for patients (Survey 3) ............................................27

Online Supplementary Table S1. Social security system arrangements in case of sick leave due to RA

Online Supplementary Table S2. Social security system arrangements in case of work disability due to RA

Online Supplementary Table S3. Regulations to prevent work disability and help staying at work for people with RA

Online Supplementary Table S4. Patients' and rheumatologists' perceptions across several domains according to (1) GDP per capita PPP (2) EU membership status (3) Type of social security system ....43 


\section{Online Supplementary Text S1a. Questionnaire administered to Principal Investigators (one rheumatologists per country, Survey 1)}

\section{Research project: "Access to social security benefits in case of sick leave or work disability in rheumatoid arthritis across European countries".}

The aim of the project is:

1. To gain insight into the differences in access to social security benefits in case of sick leave and work disability for patients with rheumatoid arthritis (RA), in terms of eligibility criteria, and

2. To compare the level of income substitution and impoverishment in different countries across Europe.

\section{Questionnaire to be completed by the principal investigator of each country}

The principal investigator of each country is asked to complete the questionnaire below. This questionnaire consists of five parts:

1. Facilities to allow continuing participation in work force and to prevent work disability

2. Regulations in case of sick leave (short-term work disability)

3. Regulations in case of long-term work disability

4. Facilities to return to work after a period of long-term disability

5. Regulations in case of disability for self-employed persons

\section{Co-authorship rules}

The Principal Investigator will be a co-author in case the journal does not set strict limitations for the number of authors. We have confirmed with ARD (journal of first choice) that $>40$ authors is allowed given that author provides sufficient contribution. In this case the co-author will become coresponsible for the information published. More than 40 countries are invited to participate. Therefore, please note that due to restrictions from journals regarding the maximum number of authors, we can include only one person per country as a co-author. If the journal allows, one additional person who has substantially contributed to the data collection process could be included as a member of the working group and up to two persons can be acknowledged at the end of the manuscript.

Instructions on how to complete the questionnaire 
Completion of some parts of the questionnaire may require inquiring experts in your country who possess the specific knowledge on regulations in respect of work disability allowances. We encourage you to make all the efforts to obtain the most reliable information, which will likely involve contacting other people you consider appropriate. We also ask you to provide the official sources of information of the obtained data, as those are essential for publication of the final manuscript.

\section{Focus of the questionnaire}

Please, keep in mind that all the questions are related to work disability due to rheumatoid arthritis and patients who have an employment contract. In view of increasing rates of self-employed in some countries, we have added a section with a few general questions on work disability rules for selfemployed patients at the end of the questionnaire.

\section{Further recommendations on completing the questionnaire}

Due to expected large differences between systems, we attempt to make a universally applicable questionnaire to cover the general topics. It can be that some questions are not applicable to the situation in your country. After each question, there is always space left for additional comments from your side, and we invite you to provide additional comments for us to understand the local system better. Always think about the most general and most applicable case. Please, disregard variations and exceptions (for example, across regions or minority groups) or, if in doubt, describe them in the field for additional comments). In case it seems that the questions do not cover sufficiently the situation in your country, please indicate this in your comments. We will be in contact with Principal Investigator and adjust the questionnaire with country specific additions, if needed.

\section{$\underline{\text { Paper and online versions of the questionnaire }}$}

It is also possible to fill in this questionnaire online. Individual link to the survey is available in the invitation letter you have received. Questions are identical in both versions of the survey, and either of two is enough to complete. Should you have difficulties in accessing the online survey, please, let us know as soon as possible.

\section{Contact information}

Polina Putrik, e-mail: polina.putrik@gmail.com 


\section{Q1 Country}

Please, indicate for which country are you completing the questionnaire:

Country name: 


\section{Definitions and terms}

Please note, that unless explicitly stated otherwise, the questions are always referring to patients with RA.

For purposes of our project (and based on collected knowledge about different social security systems), we feel we should distinguish two situations in which a patient cannot continue working because of RA:

1. Sick leave (also referred to as short-term work disability), which is absence from work due to disease starting from day 1 of the disease-related absence with a prospect to go back to the same work place after recovery.

2. Long-term work disability, which implies permanent disability to work, which generally follows a period of sickness (short-term work disability). In this case, contract with employer is usually no longer in place

\section{Additional terminology}

Degree (\%) of work disability refers to level of incapacity to work due to physical and/or mental reasons.

Degree (\%) of work ability is an alternative measure of work disability that is being used in some countries. It relates to the extent a person is able to perform work activities compared to a nondisabled person.

Q2

Are these two definitions (sick leave and long-term work disability) similar to the definitions used in your country?

\section{Yes}

No, please explain

Additional comments 


\section{Structure of the questionnaire:}

Part 1. Regulations to prevent work disability and help staying at work for people with RA ...............6

Part 2. Short- and long-term work disability regulations ...............................................................

Part 2.1 Regulations related to sick leave (short-term work disability): system-related requirements .8

Part 2.2. Regulations related to sick leave (short-term work disability): disease-related criteria for

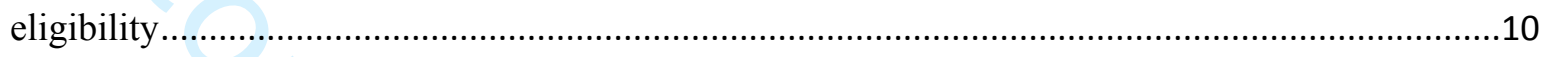

Part 2.3. Regulations related to long-term work disability: system-related requirements..................12

Part 2.4. Regulations related to long-term work disability: disease related criteria ..........................14

Part 3. Arrangements to facilitate return to work for patients with health problems due to rheumatoid

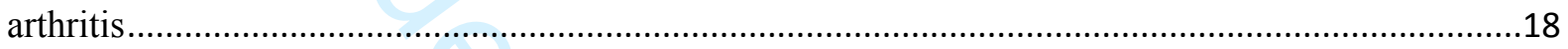

Part 4. Regulations in case of work disability for patients who are self-employed.............................19

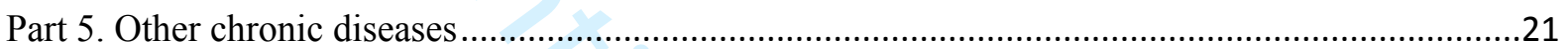




\section{Part 1. Regulations to prevent work disability and help staying at work for people with RA}






\section{Part 2. Short- and long-term work disability regulations}

This part contains questions about system-related (part 2.1) and disease-related (part 2.2) criteria for obtaining the short-term work disability (sick leave) allowance.

For example, should a person be a citizen of the country to qualify, how long the person should be employed before he or she has a right for sick leave allowance, who is authorized to certify the sick leave, etc. Please, in part 2.1 fill in the information for both permanent and temporary contracts even if similar rules apply for both.

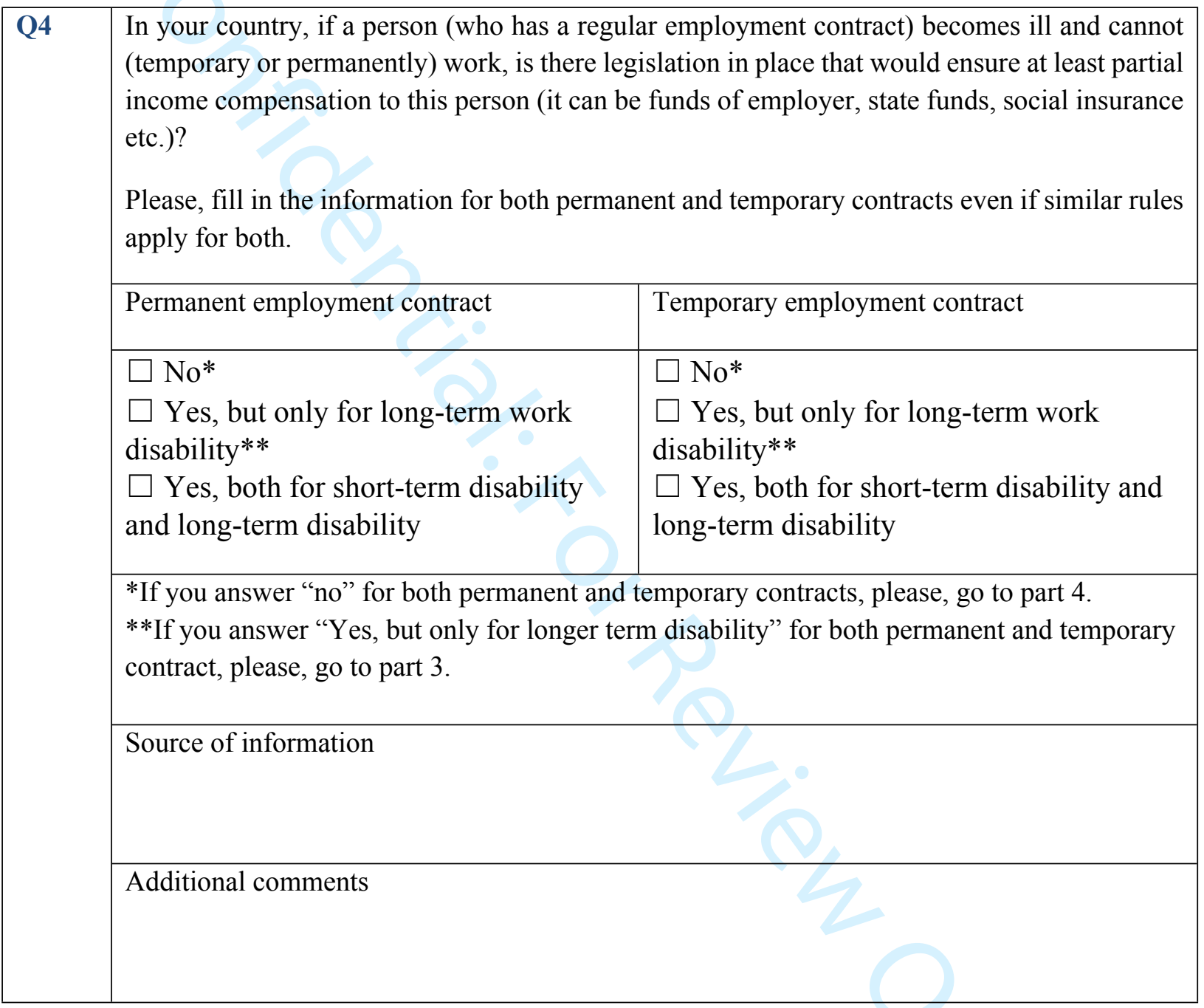


$\underline{\text { Part 2.1 Regulations related to sick leave (short-term work disability): system-related requirements }}$

\begin{tabular}{|c|c|c|}
\hline \multirow[t]{6}{*}{ Q5 } & \multicolumn{2}{|c|}{$\begin{array}{l}\text { Is there a requirement for a minimum number of years a person should reside in the country } \\
\text { to become eligible for sick leave allowance? }\end{array}$} \\
\hline & Permanent employment contract & Temporary employment contract \\
\hline & $\begin{array}{l}\square \text { No } \\
\square \text { Yes. Please, indicate the minimally } \\
\text { required period__ years } \\
\square \text { Yes, a person should be a citizen } \\
\square \text { Other. Please, explain }\end{array}$ & $\begin{array}{l}\square \text { No } \\
\square \text { Yes. Please, indicate the minimally } \\
\text { required period _ years } \\
\square \text { Yes, a person should be a citizen } \\
\square \text { Other. Please, explain }\end{array}$ \\
\hline & $\square$ Not applicable & $\square$ Not applicable \\
\hline & \multicolumn{2}{|l|}{ Source of information } \\
\hline & \multicolumn{2}{|l|}{ Additional comments } \\
\hline \multirow[t]{5}{*}{ Q6 } & \multicolumn{2}{|c|}{$\begin{array}{l}\text { Is there a requirement for the minimum length of official employment in order to be eligible } \\
\text { for a sick leave allowance? }\end{array}$} \\
\hline & Permanent employment contract & Temporary employment contract \\
\hline & $\begin{array}{l}\square \text { No } \\
\square \text { Yes, } \\
\square \text { Yes, } \\
\square \text { Yes, } \\
\square \text { workinths } \\
\square \text { Not applicable }\end{array}$ & 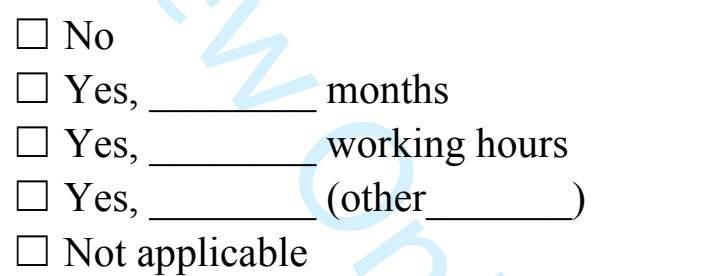 \\
\hline & \multicolumn{2}{|l|}{ Source of information } \\
\hline & Additional comments & \\
\hline
\end{tabular}




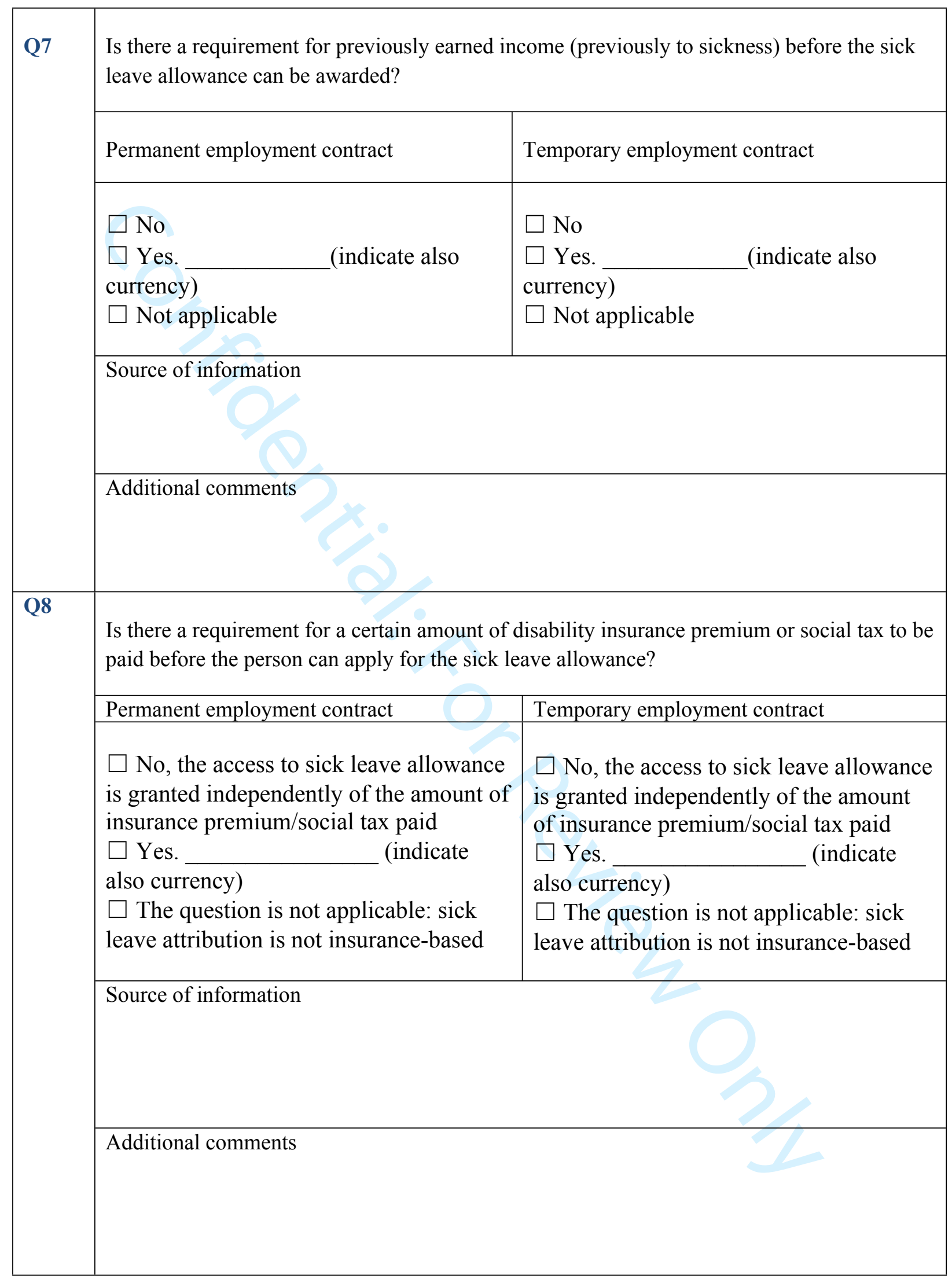


Part 2.2. Regulations related to sick leave (short-term work disability): disease-related criteria for eligibility

\begin{tabular}{|c|c|c|c|c|c|}
\hline \multirow[t]{4}{*}{ Q9 } & \multicolumn{5}{|c|}{$\begin{array}{l}\text { Is there a difference in sick leave compensation in case of permanent or temporary } \\
\text { employment contract? }\end{array}$} \\
\hline & \multicolumn{5}{|c|}{$\begin{array}{l}\square \text { No, there is no difference } \\
\square \text { Yes, the arrangement is different. Please, explain briefly }\end{array}$} \\
\hline & \multicolumn{5}{|l|}{ Source of information } \\
\hline & \multicolumn{5}{|l|}{ Additional comments } \\
\hline \multicolumn{6}{|c|}{$\begin{array}{l}\text { In case regulations for sick leave compensation are different for permanent and temporary contracts, } \\
\text { please refer in the following questions }(\mathbf{Q 1 0}-\mathbf{Q 1 4}) \text { to the conditions valid for those with a } \\
\text { permanent work contract }\end{array}$} \\
\hline \multirow[t]{11}{*}{ Q10 } & \multicolumn{5}{|c|}{$\begin{array}{l}\text { In case of RA, who can officially (i.e. legally) justify sick leave? We allow a possibility to } \\
\text { distinguish between the initial sick leave and prolonged sick leave, please, adjust the table } \\
\text { according to the reality in your country. It may be that not all columns have to be } \\
\text { completed. }\end{array}$} \\
\hline & \multirow{2}{*}{\multicolumn{2}{|c|}{ Qualification }} & $\begin{array}{l}\text { Initial sick } \\
\text { leave } \\
\text { (from day } \\
\quad \text { till day } \\
\text { ) }\end{array}$ & $\begin{array}{l}\text { First } \\
\text { prolongation } \\
\text { sick leave } \\
\text { (from } \\
\text { day_till } \\
\text { day___* }\end{array}$ & $\begin{array}{l}\text { Second } \\
\text { prolongation } \\
\text { sick leave } \\
\text { (from day } \\
\text { till day__ }\end{array}$ \\
\hline & & & & \multicolumn{2}{|c|}{ *if applicable } \\
\hline & \multicolumn{2}{|c|}{ General practitioner (GP) } & $\square$ & $\square$ & $\square$ \\
\hline & \multicolumn{2}{|l|}{ Rheumatologist } & $\square$ & $\square$ & $\square$ \\
\hline & $\begin{array}{l}\text { Physician } \\
\text { (working for public } \\
\text { or private insurance } \\
\text { company/institute) }\end{array}$ & $\begin{array}{l}\text { Specialised in } \\
\text { occupational issues: } \\
\square \text { Yes } \\
\square \text { No }\end{array}$ & $\square$ & $\square$ & $\square$ \\
\hline & $\begin{array}{l}\text { Physician } \\
\text { (working for } \\
\text { employer) }\end{array}$ & $\begin{array}{l}\text { Specialised in } \\
\text { occupational issues: } \\
\square \text { Yes } \\
\square \text { No }\end{array}$ & $\square$ & $\square$ & $\square$ \\
\hline & \multicolumn{2}{|l|}{ Other } & $\square$ & $\square$ & $\square$ \\
\hline & \multicolumn{2}{|c|}{$\begin{array}{l}\text { Should not be justified (patient can report to } \\
\text { be sick and stay at home without anyone } \\
\text { certifying the reasons) }\end{array}$} & $\square$ & $\square$ & $\square$ \\
\hline & \multicolumn{5}{|l|}{ Source of information } \\
\hline & \multicolumn{5}{|l|}{ Additional comments } \\
\hline
\end{tabular}




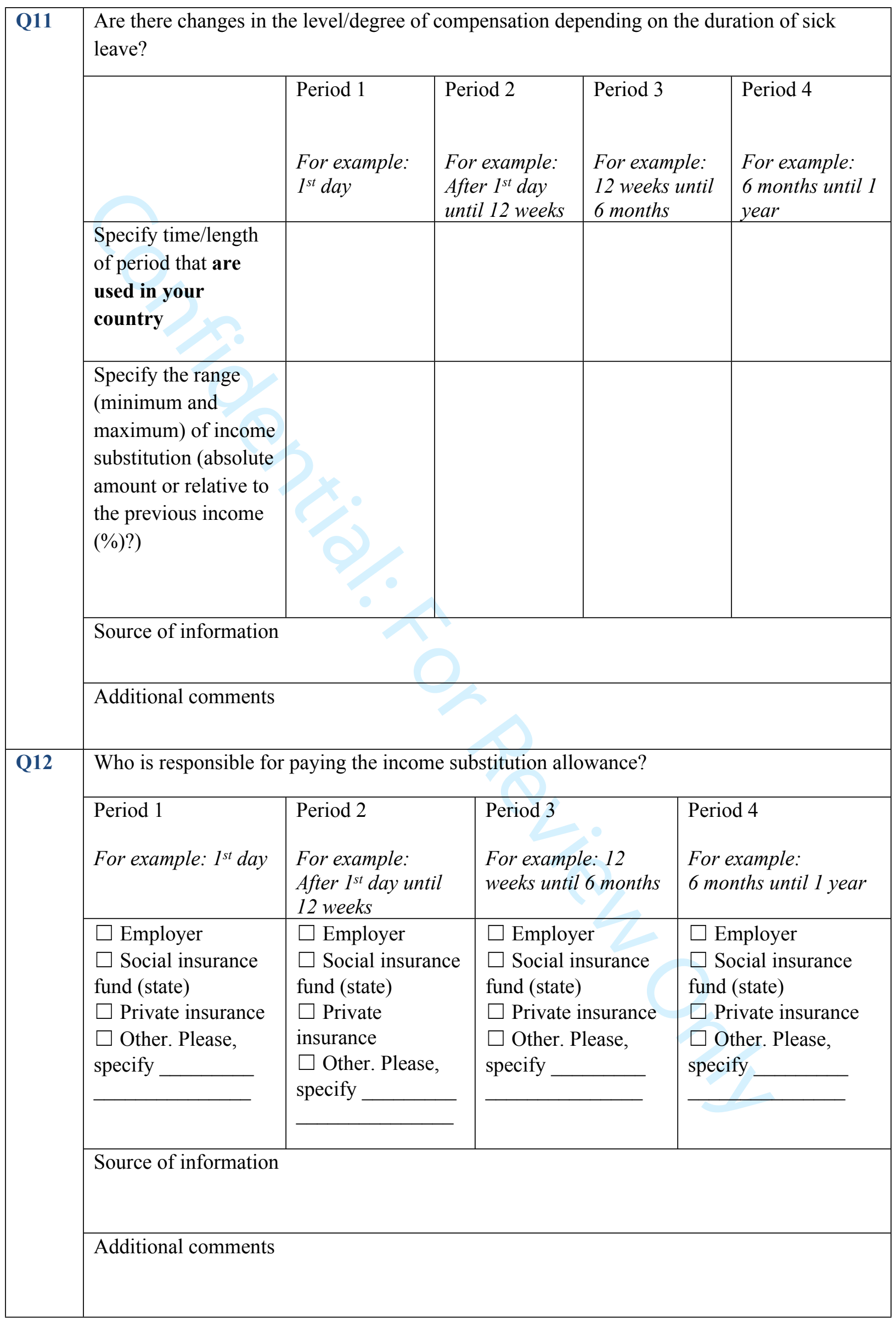




\begin{tabular}{|l|l|}
\hline Q13 & $\begin{array}{l}\text { What is the maximum duration of sick leave allowed (e.g. eligible for compensation), } \\
\text { without entering long-term disability? }\end{array}$ \\
\hline $\begin{array}{l}\square \text { There is no maximum duration of sick leave specified } \\
\square \text { Continuous sick leave of _ months } \\
\square \text { Total sick leave of } \\
\square \text { Year } \\
\square \text { Other period }\end{array}$ \\
\hline $\begin{array}{l}\text { Source of information } \\
\text { Additional comments }\end{array}$ \\
\hline
\end{tabular}

Part 2.3. Regulations related to long-term work disability: system-related requirements

Part 2.3 and 2.4 contain questions about system-related and disease-related criteria that apply when a person needs to receive long-term work disability allowance. Questions cover such topics as citizenship rules, length of employment contract, earned income etc. Further, you will be inquired about authorities that certify the disease and the fact that a person is not able to continue working and the criteria that would be taken into account when such decision is taken. Furthermore, questions about the levels of compensation (e.g. income substitution by the work disability pension) are asked. Please, in part 2.3 fill in the information for both permanent and temporary contracts even if similar rules apply for both.

\begin{tabular}{|l|l|l|}
\hline Q14 & $\begin{array}{l}\text { Is there a requirement for a minimum number of years a person should reside in the country } \\
\text { to become eligible for long-term work disability allowance? }\end{array}$ \\
\cline { 2 - 3 } & $\begin{array}{l}\text { Permanent employment contract } \\
\begin{array}{l}\square \text { No } \\
\square \text { Yes. Please, indicate the minimally } \\
\text { required period: } \\
\square \text { Yes, a person should be a citizen } \\
\square \text { Not applicable }\end{array}\end{array}$ & $\begin{array}{l}\square \text { Temporary employment contract } \\
\square \text { Yes. Please, indicate the minimally } \\
\text { required period: } \\
\square \text { Yes, a person should be a citizen } \\
\square \text { Not applicable }\end{array}$ \\
\cline { 2 - 3 } & $\begin{array}{l}\text { Source of information } \\
\text { Q15 }\end{array}$ & $\begin{array}{l}\text { Is there a requirement for the minimum length of (official) employment in order to be eligible } \\
\text { for a long-term work disability allowance? }\end{array}$ \\
\cline { 2 - 3 } & \begin{tabular}{l} 
Additional comments \\
\hline
\end{tabular}
\end{tabular}




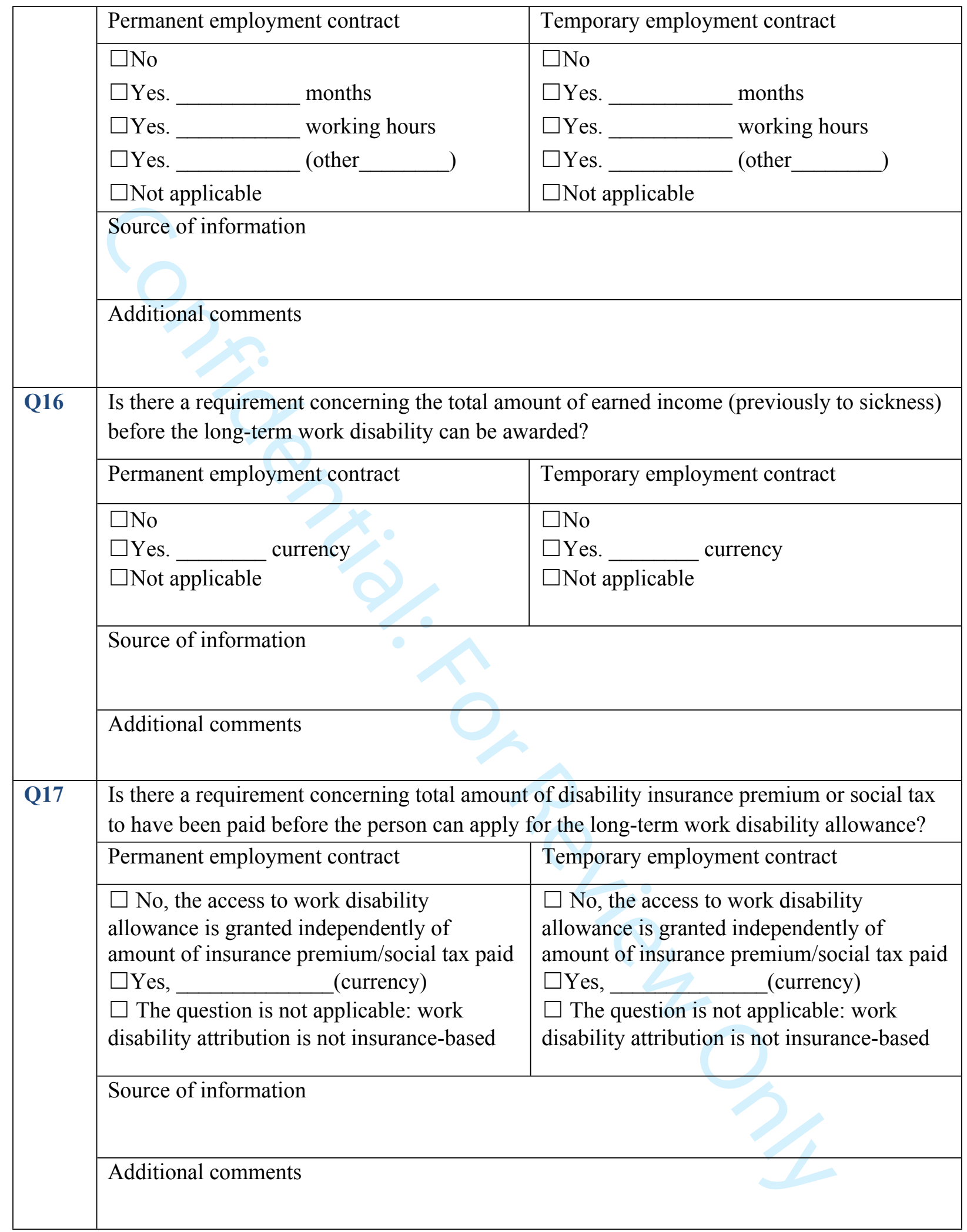


$\underline{\text { Part 2.4. Regulations related to long-term work disability: disease related criteria }}$

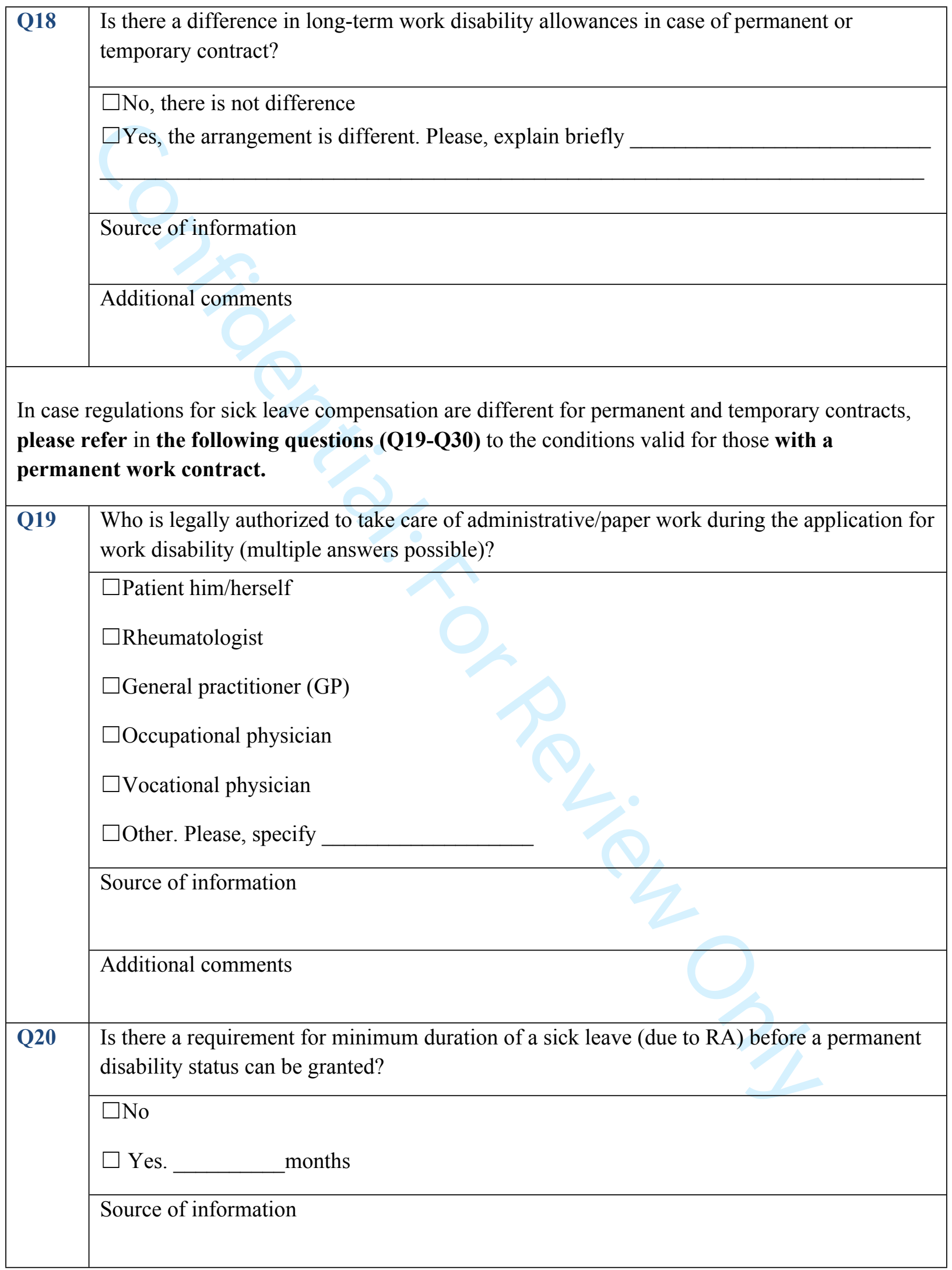




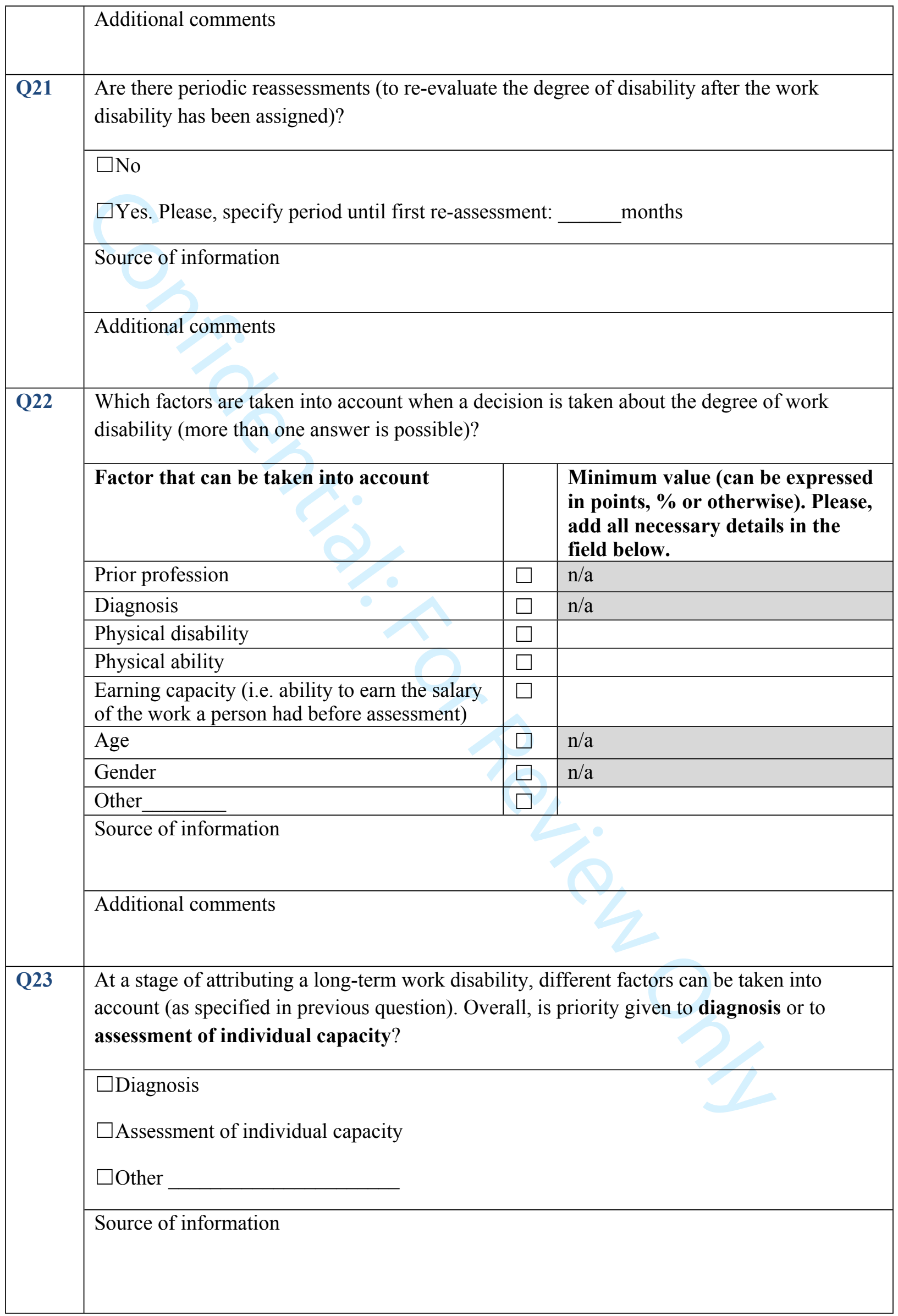




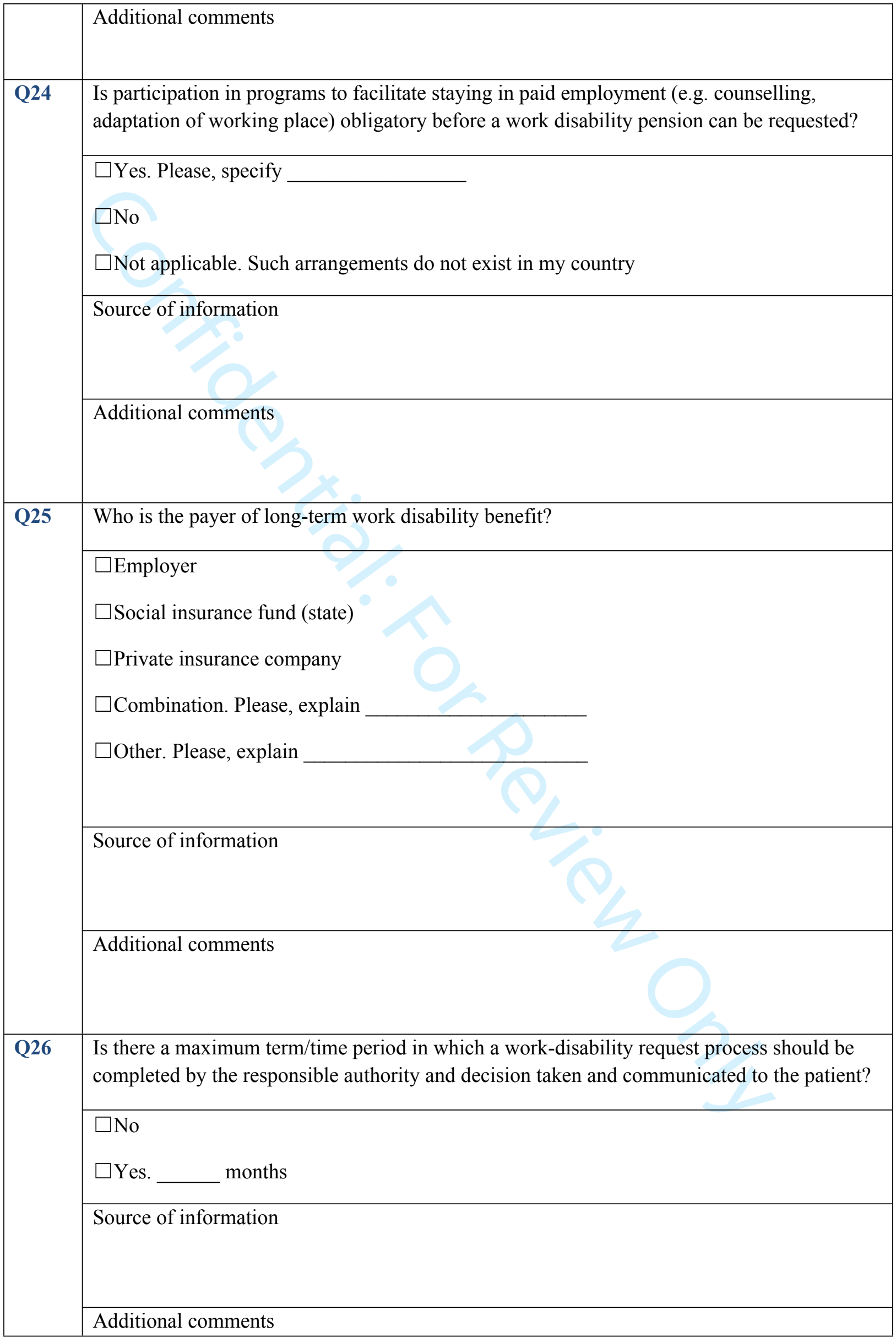




\begin{tabular}{|c|c|c|c|c|c|}
\hline \multirow[t]{6}{*}{$\begin{array}{l}\text { Q28- } \\
29-30\end{array}$} & \multicolumn{5}{|c|}{$\begin{array}{l}\text { In the table below we ask you to specify the degree of income substitution (absolute } \\
\text { amount or relative to the previous income (\%)) (range minimum-maximum). We } \\
\text { assume it may be dependent on the work (dis)ability one has. Some countries use degree of } \\
\text { work disability (for example, } 30 \%, 60 \% \text { etc.), others assess the degree of full work ability } \\
\text { (see definitions in the beginning to the questionnaire). Cut-offs differ between countries, } \\
\text { therefore we ask you to specify the ones used in your country, either in column "Degree of } \\
\text { disability" or "Degree of full work ability", depending on what applies to your country. }\end{array}$} \\
\hline & \multicolumn{2}{|c|}{$\begin{array}{l}\text { Specify relevant cut-offs used in your } \\
\text { system (it can be in \%, points or } \\
\text { another metrics; please, add necessary } \\
\text { explanations in the field below): }\end{array}$} & \multirow{2}{*}{$\begin{array}{l}\begin{array}{l}\text { Level of } \\
\text { income } \\
\text { substitution }\end{array} \\
\text { Period } 1 \\
\text { (from month } \\
\text { until } \\
\text { month }\end{array}$} & \multirow{2}{*}{$\begin{array}{l}\text { Level of } \\
\text { income } \\
\text { substitution } \\
\text { Period } 2 \\
\text { (from month } \\
\text { month } \\
\end{array}$} & \multirow{2}{*}{$\begin{array}{l}\text { Level of income } \\
\text { substitution } \\
\text { Period } 3 \\
\text { (from month } \\
\overline{\text { month until }}\end{array}$} \\
\hline & $\begin{array}{l}\text { Degree of } \\
\text { disability }\end{array}$ & $\begin{array}{l}\text { Degree of full work } \\
\text { ability }\end{array}$ & & & \\
\hline & & 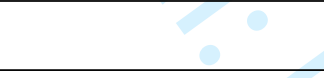 & & & \\
\hline & \multicolumn{5}{|c|}{ Source of information } \\
\hline & \multicolumn{5}{|c|}{ Additional comments } \\
\hline \multirow[t]{9}{*}{ Q31 } & \multicolumn{5}{|c|}{$\begin{array}{l}\text { In addition to income substitution specified in question Q28-29-30, does patient with work } \\
\text { disability also get a right for non-monetary benefits? (if yes, please, indicate the maximum } \\
\text { possible size of benefit, including currency if applicable): }\end{array}$} \\
\hline & \multicolumn{5}{|l|}{$\square$ No } \\
\hline & \multicolumn{5}{|c|}{$\square$ Yes. Please, specify below: } \\
\hline & \multicolumn{5}{|c|}{$\circ$ Home assistance } \\
\hline & \multicolumn{5}{|c|}{ ○ Transport allowance } \\
\hline & \multicolumn{5}{|c|}{ - Extra benefit per each dependent child } \\
\hline & \multicolumn{5}{|c|}{ O Other } \\
\hline & \multicolumn{5}{|c|}{ Source of information } \\
\hline & \multicolumn{5}{|c|}{ Additional comments } \\
\hline
\end{tabular}


Part 3. Arrangements to facilitate return to work for patients with health problems due to rheumatoid arthritis

The questions of this section refer to the possibilities of continuing with work activity by a patient who was assigned a degree of long-term work disability, and possible regulations related to this process.

\begin{tabular}{|c|c|}
\hline \multirow[t]{4}{*}{ Q32 } & $\begin{array}{l}\text { Does the person who was assigned any degree of work disability have a right to work for a } \\
\text { monetary reward (it can be regular paid employment, occasional temporary work etc.)? }\end{array}$ \\
\hline & $\begin{array}{l}\square \text { No [go to question Q34] } \\
\square \text { Yes }\end{array}$ \\
\hline & Source of information \\
\hline & Additional comments \\
\hline \multirow[t]{4}{*}{ Q33 } & Does the right to work depend on the degree of work disability? \\
\hline & $\begin{array}{l}\square \text { No } \\
\square \text { Yes. Please, explain (including the relevant cut-offs for degrees of disability) }\end{array}$ \\
\hline & Source of information \\
\hline & Additional comments \\
\hline \multirow[t]{3}{*}{ Q34 } & $\begin{array}{l}\text { Are there any forms of societal support for people with a disability that would help them to } \\
\text { get employed (example can be special quotas for employers to hire people with disability, } \\
\text { subsidies for companies that employ people with disability, programs of additional education } \\
\text { etc.) }\end{array}$ \\
\hline & $\begin{array}{l}\square \text { No, this does not exist } \\
\square \text { Yes, but this does not apply for persons with RA } \\
\square \text { Yes, it exists, and also applies for persons with RA. Please, explain }\end{array}$ \\
\hline & Source of information \\
\hline
\end{tabular}




\section{Part 4. Regulations in case of work disability for patients who are self-employed}

Previous questions were referring to regulations concerning patients who are regularly employed (i.e. do not run their own business) at the moment of becoming ill. However, in some countries, the number of self-employed people is relatively large, and it is likely that other criteria for disability allowances apply for this group. Therefore, we added the section below with several general questions about work-disability regulations for self-employed population.

\begin{tabular}{|c|c|}
\hline \multirow[t]{4}{*}{ Q35 } & $\begin{array}{l}\text { Do people who are self-employed at the moment of sickness have a right for sick leave } \\
\text { compensation in case of temporary illness (in this question, we refer to state regulations, not } \\
\text { the voluntary insurance or individual savings)? }\end{array}$ \\
\hline & $\begin{array}{l}\square \text { No } \\
\square \text { Yes }\end{array}$ \\
\hline & Source of information \\
\hline & Additional comments \\
\hline \multirow[t]{4}{*}{ Q36 } & $\begin{array}{l}\text { Are people who are self-employed eligible for applying for long-term disability pension in } \\
\text { case their condition does not allow to continue working activities (in this question, we refer to } \\
\text { state regulations, not the voluntary insurance or individual savings)? }\end{array}$ \\
\hline & $\begin{array}{l}\square \text { No [Please, go to question Q38] } \\
\square \text { Yes. After___ months since the beginning of disease }\end{array}$ \\
\hline & Source of information \\
\hline & Additional comments \\
\hline \multirow[t]{2}{*}{ Q37 } & $\begin{array}{l}\text { You have answered that a person who is self-employed at the moment of sickness is eligible } \\
\text { for applying for long-term disability benefit in case his/her condition does not allow to continue } \\
\text { working activities. How is the amount of compensation (i.e. disability pension) determined? }\end{array}$ \\
\hline & $\begin{array}{l}\square \text { Based on previous income (please, specify the rules applied, including min and max) } \\
\square \text { Fixed amount } \quad \text { (indicate currency) }\end{array}$ \\
\hline
\end{tabular}




\begin{tabular}{|c|c|}
\hline & $\square$ Other rule, please, specify \\
\hline & Source of information \\
\hline & Additional comments \\
\hline Q38 & $\begin{array}{l}\text { In case of prolonged sick leave, has a self-employed person right for work re-integration } \\
\text { scheme? }\end{array}$ \\
\hline & $\begin{array}{l}\square \text { No } \\
\square \text { Yes, please explain }\end{array}$ \\
\hline & Source of information \\
\hline & Additional comments \\
\hline Q39 & $\begin{array}{l}\text { For people who were previously self-employed and had to stop working due to RA, are there } \\
\text { any forms of societal support that would help them to get paid work (example can be special } \\
\text { quotas for employers to hire people with disability, subsidies for companies that employ people } \\
\text { with disability, programs of additional education etc.) }\end{array}$ \\
\hline & $\begin{array}{l}\square \text { No. It does not exists for people who used to be self-employed at the moment they became } \\
\text { ill } \\
\square \text { Yes, but it does not apply for persons with RA } \\
\square \text { Yes, it exists for persons who used to be self-employed, and also applies for persons with } \\
\text { RA. Please, explain }\end{array}$ \\
\hline & \\
\hline & Source of information \\
\hline & Additional comments \\
\hline
\end{tabular}




\section{Part 5. Other chronic diseases}

\begin{tabular}{|l|l|}
\hline 40 & $\begin{array}{l}\text { Are the criteria/allowances specified in the previous sections of the questionnaire applicable to } \\
\text { other conditions than RA? } \\
\square \text { No, only to RA } \\
\square \text { Yes, to all rheumatologic conditions } \\
\square \text { Yes, but only for (select the ones that are applicable): } \\
\square \text { Psoriatic arthritis } \\
\square \text { Osteoarthritis } \\
\square \text { Ankylosis spondylitis/Spondyloarthritis } \\
\square \text { Polyarthritis } \\
\square \text { Chronic back pain } \\
\square \text { Other chronic conditions, namely } \\
\end{array} \mid \begin{array}{l}\text { Source of information } \\
\text { Additional comments }\end{array}$ \\
\end{tabular}

Thank you for filling in the questionnaire! 
Online Supplementary Text S1b. Questionnaire administered to Principal Investigators (one rheumatologists per country, vignette scenario for income compensation)

\begin{tabular}{|c|c|c|c|}
\hline & \multicolumn{3}{|c|}{$\begin{array}{l}\text { Female, } 50 \text { years old, citizen of the country } \\
\text { Has } 25 \text { years of work experience } \\
\text { Works in a large private company, } 40 \text { working hours per week (equivalent to full-time work) }\end{array}$} \\
\hline $\begin{array}{l}\text { Previous income during years preceding } \\
\text { illness }\end{array}$ & $\frac{\text { Minimum salary per } 1 \text { month }}{\text { (euro/other currency }}$ & $\begin{array}{c}\frac{\text { Average salary per } 1 \text { month }}{\text { (euro/other currency }} \\
\end{array}$ & $\begin{array}{l}3 \text { times average salary per } 1 \text { month } \\
\end{array}$ \\
\hline $\begin{array}{l}\text { The patient was recently diagnosed with } \\
\text { very active RA and has not been able to } \\
\text { attend work job for } 6 \text { months (full-time sick } \\
\text { leave/short-term disability) } \\
\text { - Please, indicate actual amount of } \\
\frac{\text { income compensation that a person }}{\text { will receive during } 6 \text { months period }} \\
\text { (gross income, ie. before taxes, if any) }\end{array}$ & $\begin{array}{l}\text { Income compensation (per } 6 \text { months, as } \\
\text { described in scenario) } \\
\text {. (euro/other currency }\end{array}$ & $\begin{array}{l}\text { Income compensation (per } 6 \text { months, as } \\
\text { described in scenario) }\end{array}$ & $\begin{array}{l}\text { Income compensation (per } 6 \text { months, as described } \\
\text { in scenario) } \\
\quad \text { (euro/other currency }\end{array}$ \\
\hline $\begin{array}{l}\text { Patient has longstanding RA (10 years) and } \\
\text { was granted a year ago a permanent (long- } \\
\text { term) moderate work disability because of } \\
\text { physical incapacity to perform half }(50 \%) \text { of } \\
\text { usual work activities) }\end{array}$ & $\begin{array}{l}\text { Disability pension (per } 1 \text { year, as described in } \\
\text { scenario) }\end{array}$ & $\begin{array}{l}\text { Disability pension (per } 1 \text { year, as described in } \\
\underline{\text { scenario) }}\end{array}$ & $\begin{array}{l}\text { Disability pension (per } 1 \text { year, as described in } \\
\text { scenario) }\end{array}$ \\
\hline $\begin{array}{l}\text { - Please, indicate actual amount of } \\
\frac{\text { disability pension that a person would }}{\text { have received during first year (gross }} \\
\text { income, ie. before taxes, if any) }\end{array}$ & _____euro/other currency & _._._euro/other currency & ar currency \\
\hline
\end{tabular}




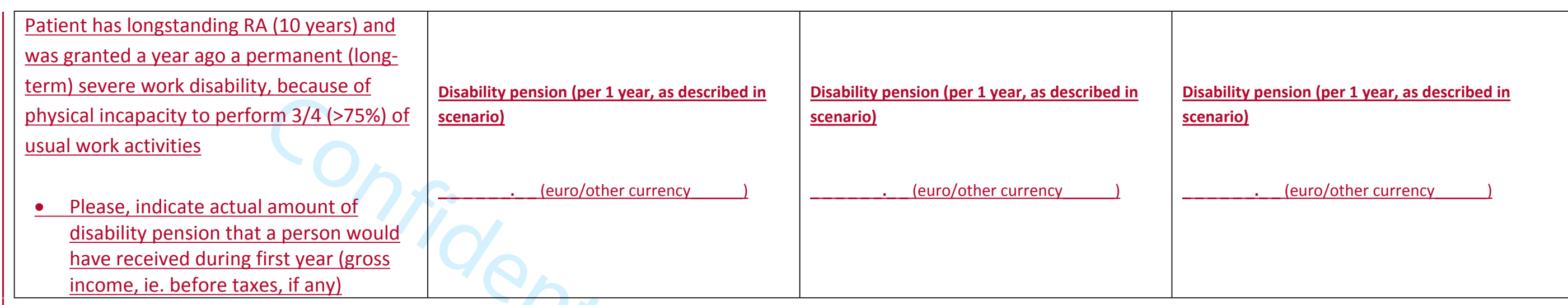




\section{Online Supplementary Text S2. Questionnaire for rheumatologists (Survey 2) \\ Perspective of rheumatologists}

\section{Acceptability of arrangements aimed at helping to stay at work or acquiring work disability pensions for patients with rheumatoid arthritis.}

This questionnaire is created to investigate opinion of rheumatologists about existing work support systems as well as regulations and attitudes in relation to work disability benefits for patient with rheumatoid arthritis in your country.

This study is part of a larger initiative to understand access to support systems and social security in case of problems to sustain paid work in patients with rheumatoid arthritis.

You have received this questionnaire because you are a rheumatologist and may have direct or indirect experience with the work participation and work disability benefits for patients with rheumatoid arthritis.

We are interested in having your opinion about different aspects related to work disability arrangements. We would like to ask you to give your opinion on the questions and statements below.

Please, note that the questionnaire is completely anonymous. Once you have completed the questionnaire you may choose among the following options to send it:

- You may send the completed version directly to the research team (Polina Putrik, polina.putrik@maastrichtuniversity.nl)

- You may return the questionnaire to the Principal Investigator in your country Dr. 
Q1 Please, indicate which country you are working in. *

*This survey is completely anonymous, and only generalizations on country level will be made during analyses stage.

1. Arrangements aimed at supporting to stay at work or acquiring work disability status for patients with rheumatoid arthritis

\begin{tabular}{|c|c|c|}
\hline Q2 & $\begin{array}{l}\text { The work related needs of patients } \\
\text { with RA are met by the (social } \\
\text { security) system in my country (i.e. } \\
\text { those who are able to work have } \\
\text { opportunity to continue working with } \\
\text { necessary adaptations) }\end{array}$ & $\begin{array}{l}\square \text { Yes, totally agree } \\
\square \text { Yes, agree } \\
\square \text { Neither agree nor disagree } \\
\square \text { No, disagree } \\
\square \text { Completely disagree } \\
\square \text { No opinion }\end{array}$ \\
\hline Q3 & $\begin{array}{l}\text { I feel that offering patient a chance } \\
\text { to stay at work (e.g. by developing a } \\
\text { work integration plan given patient's } \\
\text { reduced physical capacity) is as } \\
\text { important as offering a medical } \\
\text { treatment for his condition }\end{array}$ & $\begin{array}{l}\square \text { Yes, totally agree } \\
\square \text { Yes, agree } \\
\square \text { Neither agree nor disagree } \\
\square \text { No, disagree } \\
\square \text { Completely disagree } \\
\square \text { No opinion }\end{array}$ \\
\hline Q4 & $\begin{array}{l}\text { I feel confident and well-prepared } \\
\text { when it comes to supporting a } \\
\text { patient in maintaining/adjusting } \\
\text { his/her work duties so that he/she } \\
\text { can continue working }\end{array}$ & $\begin{array}{l}\square \text { Yes, totally agree } \\
\square \text { Yes, agree } \\
\square \text { Neither agree nor disagree } \\
\square \text { No, disagree } \\
\square \text { Completely disagree } \\
\square \text { No opinion }\end{array}$ \\
\hline Q5 & $\begin{array}{l}\text { The role of the rheumatologist in } \\
\text { my country is sufficient in the } \\
\text { process of decision making } \\
\text { regarding assigning the long-term } \\
\text { disability status for a patient }\end{array}$ & $\begin{array}{l}\square \text { Yes, totally agree } \\
\square \text { Yes, agree } \\
\square \text { Neither agree nor disagree } \\
\square \text { No, disagree } \\
\square \text { Completely disagree } \\
\square \text { No opinion }\end{array}$ \\
\hline
\end{tabular}




\begin{tabular}{|c|c|c|}
\hline Q6 & $\begin{array}{l}\text { If according to the rules in your } \\
\text { country you are the person who is } \\
\text { authorized to apply for work } \\
\text { disability pension on behalf of the } \\
\text { patient, how satisfied are you with } \\
\text { this task? }\end{array}$ & $\begin{array}{l}\square \text { I am not authorized to apply a and } \\
\text { feel comfortable with it } \\
\square \text { I am not authorized to apply a and } \\
\text { it is regrettable } \\
\square \text { I am authorized to apply and feel } \\
\text { comfortable with this role } \\
\square \text { I am authorized to apply and feel } \\
\text { uncomfortable with this role } \\
\square \text { Other }\end{array}$ \\
\hline Q7 & $\begin{array}{l}\text { If according to the rules in your } \\
\text { country you are the person who is } \\
\text { authorized to be involved in the } \\
\text { decision process for work } \\
\text { disability pension, how satisfied are } \\
\text { you with this task? }\end{array}$ & $\begin{array}{l}\square \text { I am not authorized to decide a } \\
\text { and feel comfortable with it } \\
\square \text { I am not authorized to decide a } \\
\text { and it is regrettable } \\
\square \text { I am authorized to decide and feel } \\
\text { comfortable with this role } \\
\square \text { I am authorized to decide and feel } \\
\text { uncomfortable with this role } \\
\square \text { Other }\end{array}$ \\
\hline Q8 & $\begin{array}{l}\text { The social security system is working } \\
\text { in a way that patients who need it } \\
\text { can get a work disability pension } \\
\text { (given the health status and personal } \\
\text { situation, such as family and work) }\end{array}$ & $\begin{array}{l}\square \text { Yes, totally agree } \\
\square \text { Yes, agree } \\
\square \text { Neither agree nor disagree } \\
\square \text { No, disagree } \\
\square \text { Completely disagree } \\
\square \text { No opinion }\end{array}$ \\
\hline Q9 & $\begin{array}{l}\text { How would you rate the level of } \\
\text { standardization (as opposite to } \\
\text { subjectivity) in the decision-taking } \\
\text { process regarding the long-term } \\
\text { disability? }\end{array}$ & $\begin{array}{l}\square \text { Very high level of standardization } \\
\text { (i.e. decisions are very objective) } \\
\square \text { High level of standardization (i.e. } \\
\text { decisions are objective) } \\
\square \text { Neither high nor low } \\
\square \text { Low level of standardization (i.e. } \\
\text { decisions are subjective) } \\
\square \text { Very low level of standardization } \\
\text { (i.e. decisions are very subjective) } \\
\square \text { No opinion }\end{array}$ \\
\hline Q10 & $\begin{array}{l}\text { I feel confident and well-prepared } \\
\text { when it comes to facilitating the } \\
\text { process of application for long-term } \\
\text { work disability benefit for my patients }\end{array}$ & $\begin{array}{l}\square \text { Yes, totally agree } \\
\square \text { Yes, agree } \\
\square \text { Neither agree nor disagree } \\
\square \text { No, disagree } \\
\square \text { Completely disagree } \\
\square \text { No opinion }\end{array}$ \\
\hline Q11 & $\begin{array}{l}\text { Taking all factors into account, } \\
\text { rheumatologists face a lot of }\end{array}$ & $\begin{array}{l}\square \text { Yes, totally agree } \\
\square \text { Yes, agree }\end{array}$ \\
\hline
\end{tabular}




\begin{tabular}{|l|l|l|}
\hline barriers when a patient should be & $\square$ Neither agree nor disagree \\
recognized as work disabled. & $\square$ No, disagree \\
& $\square$ Completely disagree \\
& $\square$ No opinion \\
\hline
\end{tabular}

\section{Background information about respondent}

This section contains questions about you and your work environment. This information will help us to understand and analyse your previous answers. All your responses will be anonymous, treated strictly confidential and used for the purpose of this study only.

\begin{tabular}{|c|c|c|}
\hline Q12 & What is your gender? & $\begin{array}{l}\square \text { Male } \\
\square \text { Female }\end{array}$ \\
\hline Q13 & What is your year of birth? & $19_{--}$ \\
\hline Q14 & $\begin{array}{l}\text { What best describes your current } \\
\text { work environment? (In case several } \\
\text { responses apply, please select your } \\
\text { main work environment only) }\end{array}$ & $\begin{array}{l}\square \text { University hospital (academic } \\
\text { setting) } \\
\square \text { Non-university (private or public) } \\
\text { hospital } \\
\square \text { Private practice } \\
\square \text { Other: }\end{array}$ \\
\hline Q15 & $\begin{array}{l}\text { How many years of work experience } \\
\text { as certified rheumatologist do you } \\
\text { have? }\end{array}$ & $\begin{array}{l}\square \text { Less than } 5 \text { years } \\
\square 6 \text { to } 10 \text { years } \\
\square 11 \text { to } 20 \text { years } \\
\square \text { More than } 20 \text { years }\end{array}$ \\
\hline Q16 & $\begin{array}{l}\text { What is the number of hours you } \\
\text { spend on patient care per week } \\
\text { (average)? }\end{array}$ & -- hours \\
\hline
\end{tabular}

Thank you for taking time to complete the questionnaire!

In case of questions, feel free to take direct contact with the research team: Polina Putrik, polina.putrik@maastrichtuniversity.nl or via telephone: (+31) 62478 7278 


\section{Online Supplementary Text S3. Questionnaire for patients (Survey 3) \\ Perspective of patients}

Acceptability of arrangements aimed at helping to stay at work and acquiring work disability pensions for patients with RA.

This questionnaire is created to investigate

- the attitude of persons around you towards patients who have difficulties to work due to rheumatoid arthritis

and

- the acceptability of processes and regulations related to acquiring work disability status for patients with rheumatoid arthritis

You have received this questionnaire because you are a patient with rheumatoid arthritis and may have an opinion about different aspects related to work disability, such as possibilities to continue working despite of the disease, attitude of colleagues/society to patients with rheumatoid arthritis, application process to receive a disability pension, size of payments, etc. We would like to ask you to give your opinion on the questions and statements below.

Please, note that the questionnaire is completely anonymous. Once you have completed the questionnaire you may choose among the following options to send it:

- You may send the completed version directly to the research team (Polina Putrik, polina.putrik@maastrichtuniversity.nl)

- You may return the questionnaire to the Principal Investigator in your country (Dr. Principal Investigator, PLEASE REPLACE YOUR NAME HERE BEFORE PRINTING) 
Q1 Please, indicate which country you are living in*

*This survey is completely anonymous, and only generalizations on country level will be made during analyses stage.

\section{Background information about respondent}

\begin{tabular}{|c|c|c|}
\hline Q2 & What is your gender? & $\begin{array}{l}\square \text { Male } \\
\square \text { Female }\end{array}$ \\
\hline Q3 & What is your year of birth? & 19 \\
\hline Q4 & $\begin{array}{l}\text { When did you first experience symptoms } \\
\text { of rheumatoid arthritis (year)? }\end{array}$ & $-{ }_{-}-$(indicate year) \\
\hline Q5 & $\begin{array}{l}\text { When were you diagnosed with } \\
\text { rheumatoid arthritis? }\end{array}$ & ---- (indicate year) \\
\hline Q6 & $\begin{array}{l}\text { Have you ever had to be absent from } \\
\text { work due to your rheumatoid arthritis } \\
\text { (i.e. ask for a sick leave). If yes, please } \\
\text { indicate the longest period? }\end{array}$ & $\begin{array}{l}\square \text { No } \\
\square \text { Yes. Longest period } \\
\text { was__ days }\end{array}$ \\
\hline Q7 & $\begin{array}{l}\text { Has your RA ever caused substantial } \\
\text { problems with your work that made you } \\
\text { consider applying for work disability? }\end{array}$ & $\begin{array}{l}\square \text { No } \\
\square \text { Yes }\end{array}$ \\
\hline Q8 & $\begin{array}{l}\text { What is the highest level education you } \\
\text { have ever completed? }\end{array}$ & $\begin{array}{l}\square \text { Primary school diploma } \\
\square \text { Secondary school diploma } \\
\square \text { Higher than secondary } \\
\text { diploma (e.g. (in)completed } \\
\text { university diploma) }\end{array}$ \\
\hline Q9 & $\begin{array}{l}\text { What is your current work status? (check } \\
\text { all the boxes that apply) }\end{array}$ & $\begin{array}{l}\square \text { Paid work, part- or full-time } \\
\square \text { Do not have a paid work } \\
\text { (but NOT work disabled; e.g. } \\
\text { unemployed, student, } \\
\text { housewife, retired due to } \\
\text { age) } \\
\square \text { Certified work disability } \\
\text { (full or partial) }\end{array}$ \\
\hline
\end{tabular}


2. Stay at work arrangements for patients with rheumatoid arthritis

\begin{tabular}{|c|c|c|}
\hline Q10 & $\begin{array}{l}\text { Society (it can be, for example, colleagues } \\
\text { and/or employer) has an adequate } \\
\text { understanding of what I am able and not } \\
\text { able to do in a view of physical limitations } \\
\text { resulting from my RA }\end{array}$ & $\begin{array}{l}\square \text { Yes, totally agree } \\
\square \text { Yes, agree } \\
\square \text { Neither agree nor } \\
\text { disagree } \\
\square \text { No, disagree } \\
\square \text { Completely disagree } \\
\square \text { No opinion }\end{array}$ \\
\hline Q11 & $\begin{array}{l}\text { Society perceives me as less valuable } \\
\text { because of my RA (i.e. as being less able } \\
\text { to contribute to society). }\end{array}$ & $\begin{array}{l}\square \text { Yes, totally agree } \\
\square \text { Yes, agree } \\
\square \text { Neither agree nor } \\
\text { disagree } \\
\square \text { No, disagree } \\
\square \text { Completely disagree } \\
\square \text { No opinion }\end{array}$ \\
\hline Q12 & $\begin{array}{l}\text { I feel it is personally (not considering } \\
\text { financial benefit) rewarding to be able to } \\
\text { stay at work despite the physical limitation } \\
\text { caused by RA }\end{array}$ & $\begin{array}{l}\square \text { Yes, totally agree } \\
\square \text { Yes, agree } \\
\square \text { Neither agree nor } \\
\text { disagree } \\
\square \text { No, disagree } \\
\square \text { Completely disagree } \\
\square \text { No opinion }\end{array}$ \\
\hline Q13 & $\begin{array}{l}\text { I can get sufficient support in case I } \\
\text { prefer to stay at work despite of RA (e.g. } \\
\text { my working space can be adapted, as well } \\
\text { as work duties, schedules etc.) }\end{array}$ & $\begin{array}{l}\square \text { Yes, totally agree } \\
\square \text { Yes, agree } \\
\square \text { Neither agree nor } \\
\text { disagree } \\
\square \text { No, disagree } \\
\square \text { Completely disagree } \\
\square \text { No opinion }\end{array}$ \\
\hline Q14 & $\begin{array}{l}\text { My rheumatologist/ occupational } \\
\text { physician pays sufficient attention to } \\
\text { work related problems caused by RA (i.e. } \\
\text { makes suggestions/plans on how to } \\
\text { maintain the employment given my medical } \\
\text { condition) }\end{array}$ & $\begin{array}{l}\square \text { Yes, totally agree } \\
\square \text { Yes, agree } \\
\square \text { Neither agree nor } \\
\text { disagree } \\
\square \text { No, disagree } \\
\square \text { Completely disagree } \\
\square \text { No opinion }\end{array}$ \\
\hline
\end{tabular}

3. Work disability pension for patients with rheumatoid arthritis who are not able to continue working

\begin{tabular}{|l|l|l|}
\hline Q15 & $\begin{array}{l}\text { I perceive the level of work disability } \\
\text { pension due to RA as sufficient (i.e. I do }\end{array}$ & $\begin{array}{l}\square \text { Yes, totally agree } \\
\square \text { Yes, agree }\end{array}$ \\
\hline
\end{tabular}




\begin{tabular}{|c|c|c|}
\hline & $\begin{array}{l}\text { not feel forced to stay at work in order to } \\
\text { not loose income) }\end{array}$ & $\begin{array}{l}\square \text { Neither agree nor } \\
\text { disagree } \\
\square \text { No, disagree } \\
\square \text { Completely disagree } \\
\square \text { No opinion }\end{array}$ \\
\hline Q16 & $\begin{array}{l}\text { If I am not able to work due to my RA, I } \\
\text { can obtain work disability pension } \\
\text { without particular difficulties }\end{array}$ & $\begin{array}{l}\square \text { Yes, totally agree } \\
\square \text { Yes, agree } \\
\square \text { Neither agree nor } \\
\text { disagree } \\
\square \text { No, disagree } \\
\square \text { Completely disagree } \\
\square \text { No opinion }\end{array}$ \\
\hline Q17 & $\begin{array}{l}\text { In my opinion, persons who are } \\
\text { authorized to take decision regarding } \\
\text { work disability are knowledgeable about } \\
\text { the condition, i.e. know the natural } \\
\text { history of disease with flares, and the } \\
\text { impact of fatigue etc. }\end{array}$ & $\begin{array}{l}\square \text { Yes, totally agree } \\
\square \text { Yes, agree } \\
\square \text { Neither agree nor } \\
\text { disagree } \\
\square \text { No, disagree } \\
\square \text { Completely disagree } \\
\square \text { No opinion }\end{array}$ \\
\hline Q18 & $\begin{array}{l}\text { If I apply for work disability pension, my } \\
\text { personal situation (type of work, family } \\
\text { situation) will be taken into account } \\
\text { during the decision process }\end{array}$ & $\begin{array}{l}\square \text { Yes, totally agree } \\
\square \text { Yes, agree } \\
\square \text { Neither agree nor } \\
\text { disagree } \\
\square \text { No, disagree } \\
\square \text { Completely disagree } \\
\square \text { No opinion }\end{array}$ \\
\hline Q19 & $\begin{array}{l}\text { I think my gender makes a difference for } \\
\text { the process and decision regarding the } \\
\text { work disability pension due to RA }\end{array}$ & $\begin{array}{l}\square \text { Yes, totally agree } \\
\square \text { Yes, agree } \\
\square \text { Neither agree nor } \\
\text { disagree } \\
\square \text { No, disagree } \\
\square \text { Completely disagree } \\
\square \text { No opinion }\end{array}$ \\
\hline
\end{tabular}

4. Process of applying for official work disability for patients with rheumatoid arthritis who are not able to continue working

\begin{tabular}{|l|l|l|}
\hline Q20 & $\begin{array}{l}\text { I can easily access sufficient information } \\
\text { about requirements for application for } \\
\text { work disability pension for RA patients }\end{array}$ & $\square$ Yes, totally agree \\
& $\begin{array}{l}\square \text { Neither agree nor } \\
\text { disagree } \\
\square \text { No, disagree }\end{array}$ \\
\hline
\end{tabular}




\begin{tabular}{|c|c|c|}
\hline & & $\begin{array}{l}\square \text { Completely disagree } \\
\square \text { No opinion }\end{array}$ \\
\hline Q21 & $\begin{array}{l}\text { I can easily access sufficient information } \\
\text { about levels of compensation (monetary } \\
\text { and non-monetary benefits) in case of work } \\
\text { disability }\end{array}$ & $\begin{array}{l}\square \text { Yes, totally agree } \\
\square \text { Yes, agree } \\
\square \text { Neither agree nor } \\
\text { disagree } \\
\square \text { No, disagree } \\
\square \text { Completely disagree } \\
\square \text { No opinion }\end{array}$ \\
\hline Q22 & $\begin{array}{l}\text { In my opinion, the application process in } \\
\text { order to receive disability pension due to } \\
\text { RA is not difficult }\end{array}$ & $\begin{array}{l}\square \text { Yes, totally agree } \\
\square \text { Yes, agree } \\
\square \text { Neither agree nor } \\
\text { disagree } \\
\square \text { No, disagree } \\
\square \text { Completely disagree } \\
\square \text { No opinion }\end{array}$ \\
\hline Q23 & $\begin{array}{l}\text { In my opinion, I would be treated with } \\
\text { dignity and respect if I should apply for } \\
\text { work disability pension (or I have been } \\
\text { treated with dignity and respect when } \\
\text { applying for the pension) }\end{array}$ & $\begin{array}{l}\square \text { Yes, totally agree } \\
\square \text { Yes, agree } \\
\square \text { Neither agree nor } \\
\text { disagree } \\
\square \text { No, disagree } \\
\square \text { Completely disagree } \\
\square \text { No opinion }\end{array}$ \\
\hline Q24 & $\begin{array}{l}\text { In my opinion, there is sufficient } \\
\text { confidentiality throughout the process } \\
\text { of application to work disability pension } \\
\text { due to RA }\end{array}$ & $\begin{array}{l}\square \text { Yes, totally agree } \\
\square \text { Yes, agree } \\
\square \text { Neither agree nor } \\
\text { disagree } \\
\square \text { No, disagree } \\
\square \text { Completely disagree } \\
\square \text { No opinion }\end{array}$ \\
\hline Q25 & $\begin{array}{l}\text { I think that my rheumatologist has } \\
\text { sufficient role and influence in the } \\
\text { application process for work disability } \\
\text { pension due to RA }\end{array}$ & $\begin{array}{l}\square \text { Yes, totally agree } \\
\square \text { Yes, agree } \\
\square \text { Neither agree nor } \\
\text { disagree } \\
\square \text { No, disagree } \\
\square \text { Completely disagree } \\
\square \text { No opinion }\end{array}$ \\
\hline
\end{tabular}

In case of questions, feel free to take direct contact with the research team: Polina Putrik, polina.putrik@maastrichtuniversity.nl or via telephone: +31 62478 7278

Thank you for taking time to complete the questionnaire! 
Online Supplementary Table S1. Social security system arrangements in case of sick leave due to RA

\begin{tabular}{|c|c|c|c|c|c|c|c|c|c|c|c|c|}
\hline Country & 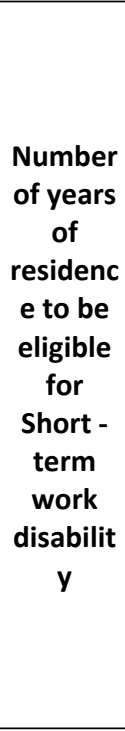 & $\begin{array}{l}\text { Length of } \\
\text { employme } \\
\text { nt to be } \\
\text { eligible for } \\
\text { Short - } \\
\text { term work } \\
\text { disability }\end{array}$ & 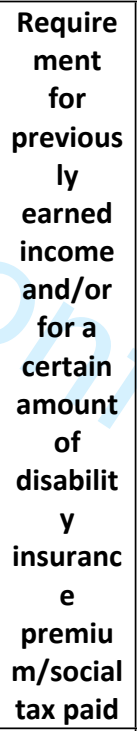 & $\begin{array}{l}\text { Who can justify sick } \\
\text { leave? }\end{array}$ & $\begin{array}{c}\text { When can } \\
\text { rheumatolog } \\
\text { ist be } \\
\text { involved in } \\
\text { certifying } \\
\text { sick leave }\end{array}$ & $\begin{array}{l}\text { When can } \\
\text { occupation } \\
\text { al therapist } \\
\text { be } \\
\text { involved in } \\
\text { certifying } \\
\text { sick leave }\end{array}$ & $\begin{array}{l}\text { When can } \\
\text { commission } \\
\text { of physicians } \\
\text { be involved } \\
\text { in certifying } \\
\text { sick-leave? }\end{array}$ & $\begin{array}{l}\text { Unpaid } \\
\text { first period } \\
\text { of sick } \\
\text { leave }\end{array}$ & $\begin{array}{l}\text { Maximum duration of } \\
\text { sick leave }\end{array}$ & $\begin{array}{c}\text { \% of } \\
\text { income } \\
\text { compensat } \\
\text { ion in first } \\
3 \text { months }\end{array}$ & $\begin{array}{c}\text { Period that } \\
\text { employer is } \\
\text { responsible } \\
\text { for (part of) } \\
\text { payment of } \\
\text { income } \\
\text { compensatio } \\
n\end{array}$ & $\begin{array}{l}\text { Is part of } \\
\text { this } \\
\text { period } \\
\text { shared } \\
\text { with } \\
\text { social } \\
\text { insuranc } \\
\text { e? }\end{array}$ \\
\hline $\begin{array}{c}\text { Albania }^{P} \\
\text { (Non-EU, Low GDP) }\end{array}$ & No & $\begin{array}{c}\text { Not } \\
\text { specified }\end{array}$ & Yes & $\begin{array}{l}\text { GP, RHEUM, physician }{ }^{4} \text {, } \\
\text { health commission }\end{array}$ & from day 1 & $\begin{array}{c}\text { from day } \\
60\end{array}$ & $\begin{array}{c}\text { from day } \\
180\end{array}$ & No & 6 months in one year & $70-80 \%$ & $\begin{array}{l}\text { first } 12 \\
\text { weeks }\end{array}$ & No \\
\hline $\begin{array}{c}\text { Austria }^{\mathrm{B}} \\
\text { (EU-15_High GDP) }\end{array}$ & No & $\begin{array}{c}\text { Not } \\
\text { specified }\end{array}$ & No & GP, RHEUM, physician ${ }^{5}$ & from day 1 & from day 1 & na & No & $\begin{array}{c}26 \text { or } 52 \text { weeks }^{7} \text {, with } \\
\text { max } 31 \text { days } \\
\text { interruption }\end{array}$ & $83 \%$ & day $4-12$ & No \\
\hline $\begin{array}{c}\text { Belarus }^{\mathrm{P}} \\
\text { (Non-EU, Low GDP) }\end{array}$ & Yes $^{1}$ & $\begin{array}{c}\text { Not } \\
\text { specified }\end{array}$ & No & GP, RHEUM, physician ${ }^{5}$ & from day 1 & na & na & No & $\begin{array}{c}120 \text { days } \\
\text { continuously or } 150 \\
\text { days intermittently } \\
\end{array}$ & $96 \%$ & $\begin{array}{c}\text { first } 6 \\
\text { months }\end{array}$ & No \\
\hline $\begin{array}{c}\text { Belgium }^{\mathrm{B}} \\
\text { (EU-15, High GDP) }\end{array}$ & No & $\begin{array}{c}1-6 \\
\text { months }\end{array}$ & No & GP, rheumat. & from day 1 & na & na & No & $\begin{array}{l}12 \text { months, max. } 3 \\
\text { months interruption }\end{array}$ & $60 \%$ & day $7-30$ & No \\
\hline $\begin{array}{c}\text { Bosnia\&Herzegovina }{ }^{P} \\
\text { (Non-EU, Low GDP) }\end{array}$ & No & $\begin{array}{c}\text { Not } \\
\text { specified }\end{array}$ & No & GP, rheumat. & from day 1 & na & na & No & 12 months & $90 \%$ & $\begin{array}{l}\text { first } 12 \\
\text { weeks }\end{array}$ & No \\
\hline $\begin{array}{c}\text { Bulgaria }^{\mathrm{P}} \\
\text { (EU-28 Low GDP) }\end{array}$ & No & $\begin{array}{c}>=6 \\
\text { months }\end{array}$ & No & $\begin{array}{l}\text { GP, hospital MD, medical } \\
\text { advisory committees, } \\
\text { territorial expert medical } \\
\text { commission }\end{array}$ & na & na & from day 30 & No & 18 months & $80 \%$ & first 3 days & No \\
\hline $\begin{array}{c}\text { Croatia }^{\mathrm{P}} \\
\text { (EU-28, Low GDP) }\end{array}$ & No & $\begin{array}{c}>=6 \\
\text { months }\end{array}$ & No & GP, physician ${ }^{5}$ & na & $\begin{array}{l}\text { from day } \\
365\end{array}$ & na & No & 12 months & $75 \%$ & first 42 days & No \\
\hline $\begin{array}{c}\text { Cyprus }^{\mathrm{M}} \\
\text { (EU-28, HighGDP) }\end{array}$ & No & $\begin{array}{c}>=6 \\
\text { months }\end{array}$ & Yes & GP, RHEUM, Physician ${ }^{6}$ & from day 1 & from day 1 & na & Yes, 3 days & 156 days & $57 \%$ & $\begin{array}{c}\text { first } 156 \\
\text { days }\end{array}$ & Yes \\
\hline
\end{tabular}




\begin{tabular}{|c|c|c|c|c|c|c|c|c|c|c|c|c|c|}
\hline 1 & $\begin{array}{l}\text { Czech Republic }^{\mathrm{P}} \\
\text { (EU-28, High GDP) }\end{array}$ & No & $\begin{array}{c}\text { Not } \\
\text { specified }\end{array}$ & Yes & GP, physician ${ }^{5}$ & from day 1 & from day 1 & na & Yes, 3 days & 12 months & $57 \%$ & day $4-14$ & No \\
\hline $\begin{array}{l}2 \\
3\end{array}$ & $\begin{array}{c}\text { Denmark }^{\mathrm{S}} \\
\text { (EU-15, High GDP) }\end{array}$ & No & $\begin{array}{c}1-6 \\
\text { months }\end{array}$ & No & RHEUM, physician ${ }^{5}$ & from day 1 & na & na & No & 3 years & $100 \%$ & $\begin{array}{c}\text { first } 22 \\
\text { weeks }\end{array}$ & Yes \\
\hline $\begin{array}{l}4 \\
5 \\
6 \\
7 \\
8\end{array}$ & $\begin{array}{c}\text { Eestonia }^{\mathrm{P}} \\
(\text { EU-28, Low GDP) }\end{array}$ & No & $\begin{array}{c}1-6 \\
\text { months }\end{array}$ & No & GP, physician ${ }^{5}$ & from day 1 & $\begin{array}{c}\text { from day } \\
121\end{array}$ & na & Yes & $\begin{array}{l}\text { Continuous sick leave } \\
\text { of } 182 \text { days or total } \\
\text { sick leave of } 250 \text { days } \\
\text { (e.g. several periods } \\
\text { of sick leave) }\end{array}$ & $67 \%$ & day $4-8$ & No \\
\hline $\begin{array}{l}9 \\
10 \\
11\end{array}$ & $\begin{array}{c}\text { Finland }^{\mathrm{S}} \\
\text { (EU-15, High GDP) }\end{array}$ & No & $\begin{array}{c}\text { Not } \\
\text { specified }\end{array}$ & No & GP, RHEUM, physician ${ }^{6}$ & from day 1 & from day 1 & na & No & $\begin{array}{c}12 \text { months per } 2 \\
\text { years }\end{array}$ & \begin{tabular}{|c|} 
not \\
possible to \\
define $\%$
\end{tabular} & First 56 days & Yes \\
\hline $\begin{array}{l}12 \\
13 \\
14 \\
15\end{array}$ & $\begin{array}{c}\text { France }^{\mathrm{B}} \\
\text { (EU-15, High GDP) }\end{array}$ & No & $\begin{array}{c}1-6 \\
\text { months }\end{array}$ & Yes & GP, RHEUM & from day 1 & na & na & Yes, 3 days & 360 days per 3 years & $76 \%$ & $\begin{array}{c}\text { from day } 4, \\
\text { max. } 360 \\
\text { days per } 3 \\
\text { years } \\
\end{array}$ & Yes \\
\hline $\begin{array}{l}16 \\
17\end{array}$ & $\begin{array}{c}\text { Georgia }^{\mathrm{P}} \\
\text { (Non-EU, Low GDP) }\end{array}$ & na & $\begin{array}{c}\text { Not } \\
\text { specified }\end{array}$ & $\mathrm{n} / \mathrm{a}$ & $\mathrm{n} / \mathrm{a}$ & $\mathrm{n} / \mathrm{a}$ & $\mathrm{n} / \mathrm{a}$ & $\mathrm{n} / \mathrm{a}$ & $\mathrm{n} / \mathrm{a}$ & $\mathrm{n} / \mathrm{a}$ & $\mathrm{n} / \mathrm{a}$ & $\mathrm{n} / \mathrm{a}$ & $\mathrm{n} / \mathrm{a}$ \\
\hline $\begin{array}{l}18 \\
19 \\
20 \\
21 \\
22\end{array}$ & $\begin{array}{c}\text { Germany }{ }^{\mathrm{B}} \\
\text { (EU-15, High GDP) }\end{array}$ & No & $\begin{array}{c}\text { Not } \\
\text { specified }\end{array}$ & No & GP, RHEUM & from day 3 & na & na & No & 78 weeks per 3 years & $\begin{array}{c}70 \% \text { of } \\
\text { brutto or } \\
90 \% \text { of } \\
\text { netto } \\
\text { income } \\
\end{array}$ & first 45 days & No \\
\hline $\begin{array}{l}23 \\
24 \\
25 \\
26\end{array}$ & $\begin{array}{c}\text { Greece }^{\mathrm{M}} \\
\text { (EU-15, Low GDP) }\end{array}$ & No & $\begin{array}{c}1-6 \\
\text { months }\end{array}$ & Yes & GP, RHEUM, physician ${ }^{6}$ & From day 1 & from day 1 & Day 16 & No & $\begin{array}{c}\text { maximum of sick- } \\
\text { leave in months equal } \\
\text { to the sum of years in } \\
\text { work }\end{array}$ & \begin{tabular}{|c|} 
not \\
possible to \\
define $\%$
\end{tabular} & day $1-25$ & Yes \\
\hline $\begin{array}{l}27 \\
28 \\
29 \\
30\end{array}$ & $\begin{array}{c}\text { Hungary }^{P} \\
\text { (EU-28, Low GDP) }\end{array}$ & No & $\begin{array}{c}\text { Not } \\
\text { specified }\end{array}$ & No & $\begin{array}{c}\text { GP, RHEUM, Supervisors } \\
\text { of the National Health } \\
\text { Insurance Fund } \\
\text { Administration }\end{array}$ & from day 1 & na & from day 30 & No & 370 days & $60 \%$ & day $1-380$ & Yes \\
\hline $\begin{array}{l}31 \\
32 \\
33\end{array}$ & $\begin{array}{c}\text { Iceland }^{\text {S }} \\
\text { (Non-EU, High GDP) }\end{array}$ & No & $\begin{array}{c}\text { Depends } \\
\text { on work } \\
\text { union } \\
\end{array}$ & No & GP, RHEUM & from day 1 & na & na & No & 48 months & $80-100 \%$ & $\begin{array}{c}\text { first } 3 \\
\text { months }\end{array}$ & No \\
\hline $\begin{array}{l}30 \\
34 \\
35 \\
36\end{array}$ & $\begin{array}{c}\text { Ireland }^{\mathrm{A}} \\
\text { (EU-15, High GDP) }\end{array}$ & Yes $^{2}$ & $\begin{array}{c}>=6 \\
\text { months }\end{array}$ & Yes & GP, RHEUM, Physician ${ }^{6}$ & from day 3 & from day 3 & na & No & 24 months & \begin{tabular}{|c|} 
not \\
possible to \\
define $\%^{8}$ \\
\end{tabular} & $\begin{array}{c}\text { day } 7 \text { - } 1 \\
\text { year }\end{array}$ & Yes \\
\hline $\begin{array}{l}36 \\
37 \\
30\end{array}$ & $\begin{array}{c}\text { Israel }^{\mathrm{B}} \\
\text { (Non-EU, High GDP) }\end{array}$ & No & $\begin{array}{c}1-6 \\
\text { months }\end{array}$ & No & GP, RHEUM & from day 1 & na & na & Yes, 1 day & 3 months & $98 \%$ & first 90 days & Yes \\
\hline $\begin{array}{l}38 \\
39\end{array}$ & $\begin{array}{c}\text { Italy } \\
\text { (EU-15, High GDP) }\end{array}$ & No & $\begin{array}{c}\text { Not } \\
\text { specified }\end{array}$ & No & GP & na & na & na & Yes, 3 days & 6 months & $64 \%$ & first 90 days & No \\
\hline
\end{tabular}




\begin{tabular}{|c|c|c|c|c|c|c|c|c|c|c|c|c|c|}
\hline $\begin{array}{l}1 \\
2\end{array}$ & $\begin{array}{c}\text { Latvia }^{P} \\
\text { (EU-28, Low GDP) }\end{array}$ & No & $\begin{array}{c}\text { Not } \\
\text { specified }\end{array}$ & No & $\begin{array}{c}\text { GP, The Expert Medical } \\
\text { Commission }\end{array}$ & na & na & $\begin{array}{l}\text { from week } \\
26\end{array}$ & Yes, 1 day & $\begin{array}{c}26 \text { weeks, or } 52 \\
\text { weeks of the three } \\
\text { year period }\end{array}$ & $74 \%$ & day $2-10$ & No \\
\hline $\begin{array}{l}3 \\
4 \\
5 \\
6\end{array}$ & $\begin{array}{c}\text { Lithuania }^{\mathrm{P}} \\
\text { (EU-28, Low GDP) }\end{array}$ & No & $\begin{array}{c}1-6 \\
\text { months }\end{array}$ & No & $\begin{array}{l}\text { GP, RHEUM, physician } \\
\text { working at institution } \\
\text { which has contact with } \\
\text { Social Insurance Fund }\end{array}$ & From day 1 & na & na & No & $\begin{array}{c}\text { Continuous sick leave } \\
\text { of } 122 \text { days or Total } \\
\text { sick leave of } 153 \text { days } \\
\text { per year }\end{array}$ & $77 \%$ & first 2 days & No \\
\hline $\begin{array}{l}7 \\
8 \\
9 \\
10\end{array}$ & $\begin{array}{c}\text { Luxemburg }^{\text {B }} \\
\text { (EU-15, High GDP) }\end{array}$ & No & $\begin{array}{c}\text { Not } \\
\text { specified }\end{array}$ & No & $\begin{array}{l}\text { GP, RHEUM, any MD } \\
\text { approved by ministry of } \\
\text { health and not working } \\
\text { for social security }\end{array}$ & from day 1 & na & na & No & 12 months & $100 \%$ & first 90 days & No \\
\hline $\begin{array}{l}11 \\
12 \\
13\end{array}$ & $\begin{array}{l}\text { FYR MacedoniaP } \\
\text { (Non-EU, Low) }\end{array}$ & No & $\begin{array}{c}\text { Not } \\
\text { specified }\end{array}$ & No & $\begin{array}{c}\text { GP, RHEUM, Team } \\
\text { (Commission) of Three } \\
\text { Rheumatologists }\end{array}$ & from day 21 & na & from day 60 & No & 9 months & $87 \%$ & first 30 days & No \\
\hline $\begin{array}{l}14 \\
15 \\
16\end{array}$ & $\begin{array}{c}\text { MaltaM } \\
\text { (EU-28, High GDP) }\end{array}$ & No & $\begin{array}{c}\text { Not } \\
\text { specified }\end{array}$ & No & $\begin{array}{c}\text { GP, RHEUM, any } \\
\text { physician working in } \\
\text { public sector }\end{array}$ & from day 1 & na & na & No & 156 days per year & $50 \%$ & first 3 days & No \\
\hline $\begin{array}{l}17 \\
18 \\
19 \\
20\end{array}$ & $\begin{array}{c}\text { Moldova }^{\mathrm{P}} \\
\text { (Non-EU, Low GDP) }\end{array}$ & No & $\begin{array}{c}>=6 \\
\text { months }\end{array}$ & No & $\begin{array}{c}\text { GP, Medical Expertise } \\
\text { Committee (GP, RHEUM, } \\
\text { head of medical unit) }\end{array}$ & na & na & from day 7 & No & $\begin{array}{l}\text { Total sick leave of } \\
\text { calendar } 210 \text { days } \\
\text { (e.g. several periods } \\
\text { of sick leave) per }\end{array}$ & $60-90 \%$ & first 5 days & No \\
\hline $\begin{array}{l}21 \\
22\end{array}$ & $\begin{array}{c}\text { Montenegro }{ }^{\mathrm{P}} \\
\text { (Non-EU, Low GDP) }\end{array}$ & No & $\begin{array}{c}\text { Not } \\
\text { specified }\end{array}$ & No & $\begin{array}{l}\text { GP, RHEUM, sick leave } \\
\text { grant commissions }\end{array}$ & from day 1 & na & from day 31 & No & 10 months & $70 \%$ & first 60 days & No \\
\hline 23 & $\begin{array}{c}\text { Norway }^{\mathrm{s}} \\
\text { (Non-EU, High GDP) }\end{array}$ & No & $\begin{array}{c}1-6 \\
\text { months }\end{array}$ & No & $\begin{array}{l}\text { GP, RHEUM, physician } \\
\text { (working for employer) }\end{array}$ & from day 1 & from day 1 & na & No & 12 months & $100 \%$ & first 16 days & No \\
\hline $\begin{array}{l}25 \\
26 \\
27\end{array}$ & $\begin{array}{c}\text { PoLand } \\
\text { (EU-28, Low GDP) }\end{array}$ & No & $\begin{array}{c}1-6 \\
\text { months }\end{array}$ & No & $\begin{array}{c}\text { Any MD authorized by } \\
\text { the Social Insurance } \\
\text { Institution }\end{array}$ & from day 1 & na & na & No & $\begin{array}{c}182 \text { days ( } 6 \text { months) } \\
\text { per } 1 \text { year }\end{array}$ & $80 \%$ & first 30 days & No \\
\hline $\begin{array}{l}28 \\
29 \\
30\end{array}$ & $\begin{array}{c}\text { PortugalM } \\
\text { (EU-15, High GDP) }\end{array}$ & No & $\begin{array}{c}1-6 \\
\text { months }\end{array}$ & No & $\begin{array}{l}\text { GP, RHEUM, any } \\
\text { physician working in } \\
\text { public sector }\end{array}$ & from day 1 & na & na & Yes, 3 days & $\begin{array}{c}1095 \text { days/ } 36 \\
\text { months }\end{array}$ & $56 \%$ & $\begin{array}{c}\text { Emploer } \\
\text { does not } \\
\text { contribute }\end{array}$ & na \\
\hline $\begin{array}{l}31 \\
32\end{array}$ & $\begin{array}{c}\text { Romania }^{\mathrm{P}} \\
\text { (EU-28, Low GDP) }\end{array}$ & No & $\begin{array}{c}1-6 \\
\text { months }\end{array}$ & No & GP, RHEUM, physician ${ }^{6}$ & from day 1 & from day 1 & na & No & 6 months in one year & $75 \%$ & first 5 days & No \\
\hline 33 & $\begin{array}{c}\text { RussiaP }^{\mathrm{P}} \\
\text { (Non-EU,_Low GDP) }\end{array}$ & No & $\begin{array}{c}\text { Not } \\
\text { specified }\end{array}$ & No & GP, RHEUM & from day 1 & na & na & No & 12 months & $60-100 \%^{7}$ & first 3 days & na \\
\hline $\begin{array}{l}24 \\
35 \\
36 \\
27\end{array}$ & $\begin{array}{c}\text { Serbia }^{P} \\
\text { (Non-EU, Low GDP) }\end{array}$ & No & $\begin{array}{c}\text { Not } \\
\text { specified }\end{array}$ & No & $\begin{array}{c}\text { GP, Board (Consilium of } 3 \\
\text { physicians) }\end{array}$ & na & na & $\begin{array}{l}\text { data not } \\
\text { provided }\end{array}$ & No & $\begin{array}{c}\text { There is no maximum } \\
\text { duration of sick leave } \\
\text { specified }\end{array}$ & $70-100 \%$ & first 4 weeks & No \\
\hline 38 & $\begin{array}{c}\text { SlovakiaP } \\
\text { (EU-28, Low GDP) }\end{array}$ & No & $\begin{array}{c}1-6 \\
\text { months }\end{array}$ & Yes & $\begin{array}{c}\text { GP, Physician specialized } \\
\text { in occupational issues }\end{array}$ & na & week 52 & na & No & 12 months & $54 \%$ & first 10 days & no \\
\hline
\end{tabular}




\begin{tabular}{|c|c|c|c|c|c|c|c|c|c|c|c|c|c|}
\hline 1 & $\begin{array}{c}\text { Slovenia }^{\mathrm{P}} \\
\text { (EU-28, High GDP) }\end{array}$ & No & $\begin{array}{c}\text { Not } \\
\text { specified }\end{array}$ & No & GP, Physician ${ }^{5}$ & na & $\begin{array}{c}\text { from day } \\
30\end{array}$ & na & No & 12 months & $80 \%$ & first 30 days & No \\
\hline & $\begin{array}{c}\text { Spain }^{\mathrm{M}} \\
\text { (EU-15, High GDP) }\end{array}$ & No & $\begin{array}{c}1-6 \\
\text { months }\end{array}$ & No & GP, rheumat. & From day 1 & na & na & Yes, 3 days & $\begin{array}{c}545 \text { calendar days, or } \\
18 \text { months }\end{array}$ & $70 \%$ & day $4-15$ & No \\
\hline 5 & $\begin{array}{c}\text { Sweden }^{\mathrm{S}} \\
\text { (EU-15, High GDP) }\end{array}$ & No & $\begin{array}{c}>=6 \\
\text { months }\end{array}$ & No & GP, RHEUM, Physician ${ }^{6}$ & from day 8 & \begin{tabular}{|c|} 
From day \\
8 \\
\end{tabular} & na & Yes, 1 day & 24 months & $79 \%$ & day $2-14$ & No \\
\hline 8 & $\begin{array}{c}\text { Switzerland } \\
\text { (Non-EU, High GDP) }\end{array}$ & No & $\begin{array}{c}\text { Not } \\
\text { specified }\end{array}$ & No & GP, rheumat. & from day 1 & na & na & No & $\begin{array}{l}730 \text { days during a } \\
\text { period of } 900 \text { days }\end{array}$ & $\begin{array}{c}\text { not } \\
\text { possible to } \\
\text { define } \% \\
\end{array}$ & first year & Yes \\
\hline $\begin{array}{l}10 \\
11 \\
12 \\
13 \\
14\end{array}$ & $\begin{array}{c}\text { TaJikistan } \\
\text { (Non-EU, Low GDP) }\end{array}$ & Yes $^{1}$ & $\begin{array}{c}\text { Not } \\
\text { specified }\end{array}$ & No & $\begin{array}{l}\text { GP, RHEUM, the vice } \\
\text { director of the medical } \\
\text { institution together with } \\
\text { the family medical } \\
\text { doctor, rheumatologist } \\
\text { and/or main specialist }\end{array}$ & from day 7 & na & from day 7 & No & $\begin{array}{l}\text { Continuous sick leave } \\
\text { of } 120 \text { days or } \\
\text { interrupted sick leave } \\
\text { for } 150 \text { days during } \\
\text { past } 12 \text { month }\end{array}$ & $30-80 \%$ & first 14 days & No \\
\hline $\begin{array}{l}15 \\
16 \\
17\end{array}$ & $\begin{array}{l}\text { The Netherlands }{ }^{B} \\
\text { (EU-15, High GDP) }\end{array}$ & No & $\begin{array}{c}\text { Not } \\
\text { specified }\end{array}$ & No & $\begin{array}{c}\text { Physician (working for } \\
\text { employer) specialized in } \\
\text { occupational issues }\end{array}$ & na & from day 1 & na & No & 24 months & $100 \%$ & first 2 years & No \\
\hline $\begin{array}{l}18 \\
19 \\
20\end{array}$ & $\begin{array}{c}\text { TurkeyM } \\
\text { (Non-EU, Low GDP) }\end{array}$ & $\mathrm{No}^{3}$ & $\begin{array}{c}\text { Not } \\
\text { specified }\end{array}$ & No & $\begin{array}{l}\text { GP, RHEUM, physician }{ }^{6} \\
\text { health commission }\end{array}$ & from day 1 & na & from day 40 & Yes, 3 days & 12 months & $63 \%$ & $\begin{array}{c}\text { Emploer } \\
\text { does not } \\
\text { contribute } \\
\end{array}$ & na \\
\hline $\begin{array}{l}21 \\
22 \\
23\end{array}$ & $\begin{array}{c}\text { Great Britain }{ }^{\mathrm{A}} \\
\text { (EU-15, High GDP) }\end{array}$ & No & $\begin{array}{c}\text { Not } \\
\text { specified }\end{array}$ & Yes & GP & na & na & na & No & $\begin{array}{c}28 \text { weeks (approx. } 7 \\
\text { months) }\end{array}$ & $\begin{array}{c}\text { not } \\
\text { possible to } \\
\text { define } \%^{8}\end{array}$ & $\begin{array}{l}\text { first } 28 \\
\text { weeks }\end{array}$ & No \\
\hline $\begin{array}{l}24 \\
25 \\
26 \\
27\end{array}$ & $\begin{array}{c}\text { Ukraine }^{\mathrm{P}} \\
\text { (Non-EU, Low GDP) }\end{array}$ & No & $\begin{array}{c}\text { Not } \\
\text { specified }\end{array}$ & No & RHEUM, physician ${ }^{5}$ & from day 1 & $\begin{array}{l}\text { from day } \\
90\end{array}$ & na & No & $\begin{array}{c}\text { Continuous sick-leave } \\
\text { of } 3 \text { months or total } 4 \\
\text { months of sick-leave } \\
\text { during one year }\end{array}$ & $50-100 \%^{7}$ & $\begin{array}{c}\text { Emploer } \\
\text { does not } \\
\text { contribute }\end{array}$ & na \\
\hline
\end{tabular}

A - Anglo-saxon, S - Scandinavian, B - Bismarkian, M - Mediterranean, P - Post-communist

EU - European Union, GDP - Gross Domestic Product (classification based on GDP per capita in international dollars), MD - medical doctor, GP - general practitioner, RHEUM - rheumatologist

$\mathrm{Na}-$ not applicable

${ }^{1}$ a person should be a citizen

2 Minimum 2 years

${ }^{3}$ but only citizen can get permanent contract)

${ }^{4}$ working for employer or public or private insurance institute and specialized in occupational issues

${ }^{5}$ working for public or private insurance company/institute

${ }^{6}$ working for public or private insurance company/institute or for employer

${ }^{7}$ depending on the work experience

8 fixed level of payment 
Online Supplementary Table S2. Social security system arrangements in case of work disability due to RA

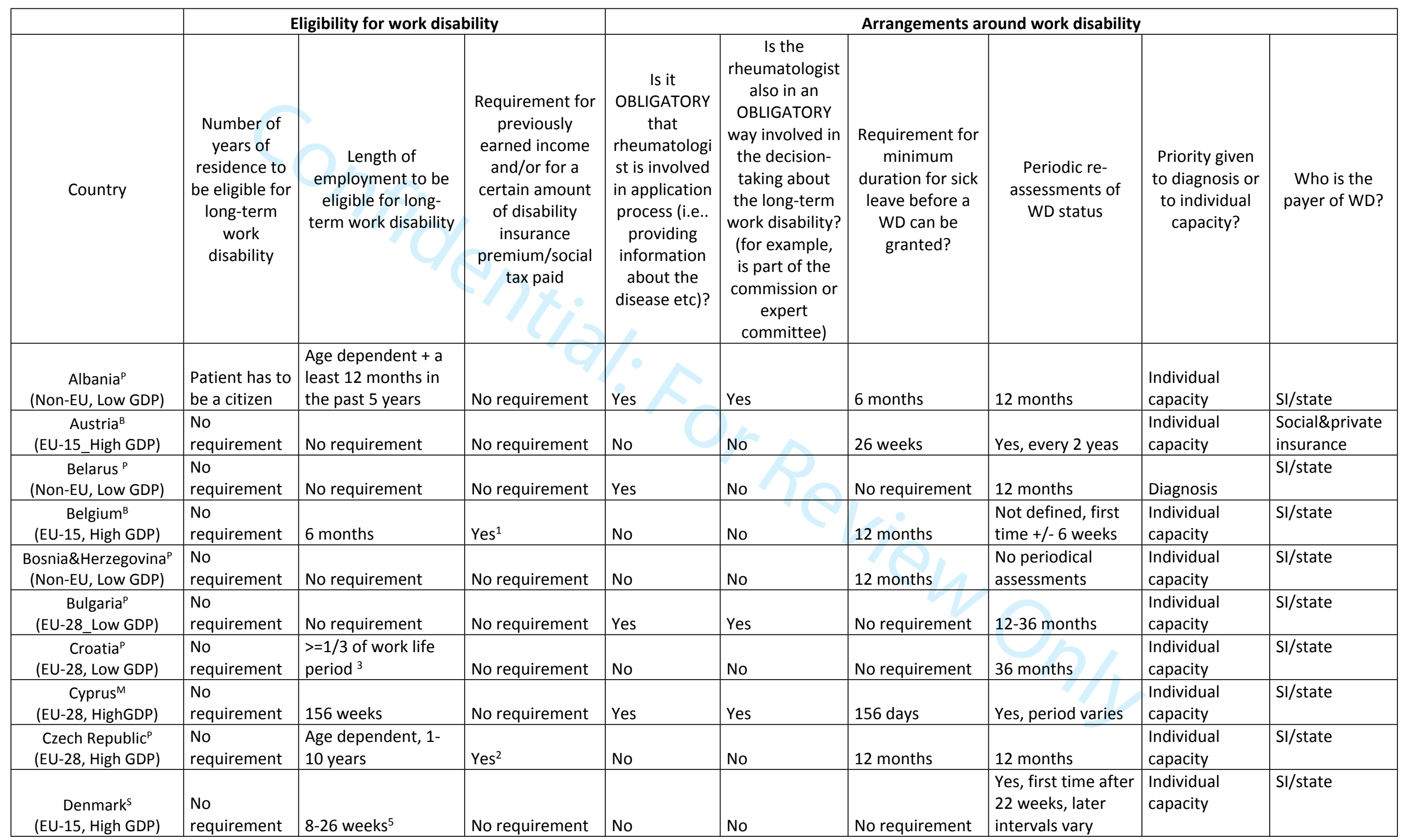




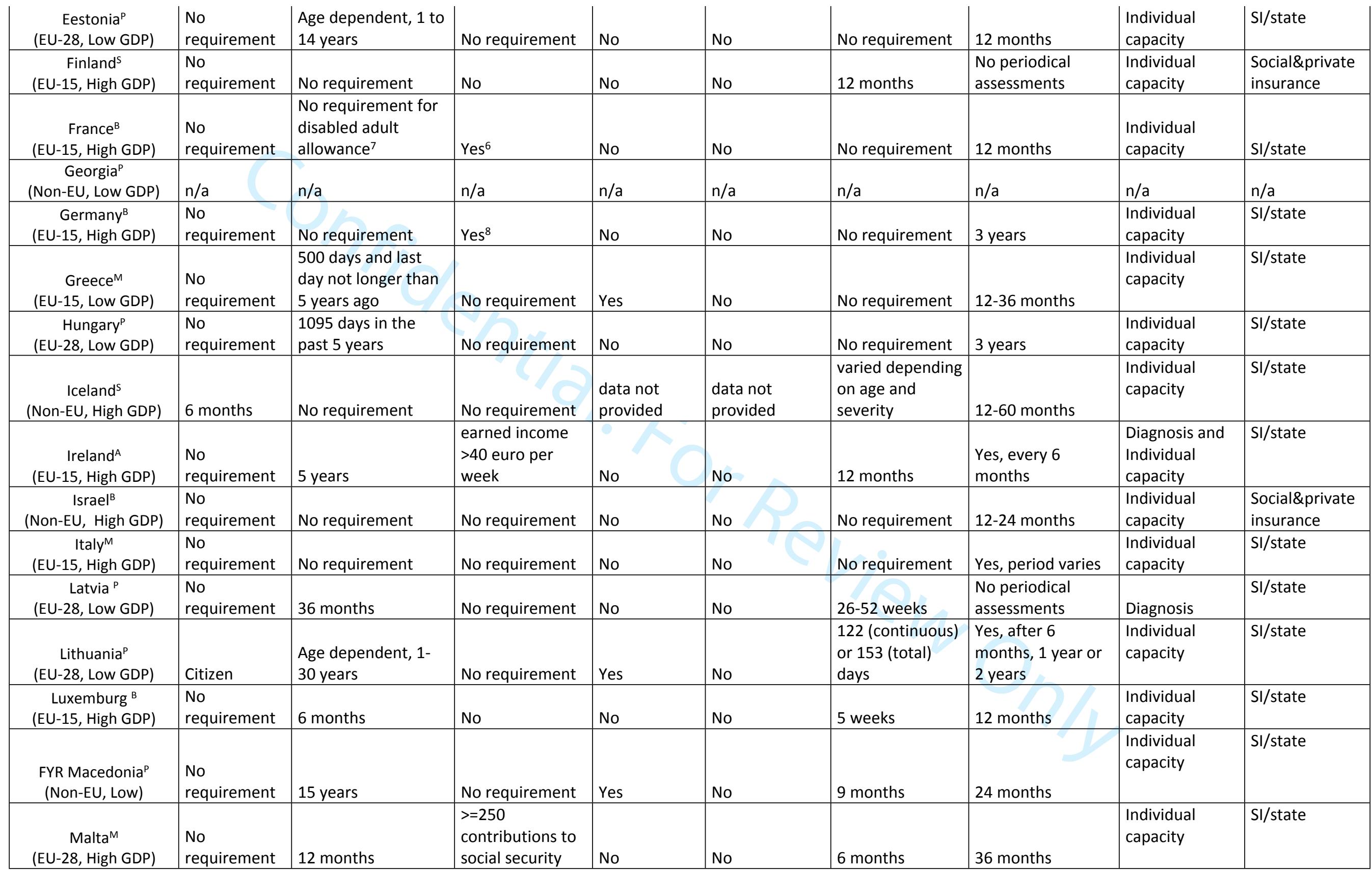









\begin{tabular}{|c|c|c|c|c|c|c|c|c|c|}
\hline $\begin{array}{c}\text { TaJikistan } \\
\text { (Non-EU, Low GDP) }\end{array}$ & Citizen & No requirement & No requirement & Yes & Yes & 120 working days & $\begin{array}{l}\text { Yes, every } 11 \\
\text { months }\end{array}$ & $\begin{array}{l}\text { Individual } \\
\text { capacity }\end{array}$ & SI/state \\
\hline $\begin{array}{l}\text { The Netherlands } \\
\text { (EU-15, High GDP) }\end{array}$ & $\begin{array}{l}\text { No } \\
\text { requirement }\end{array}$ & No requirement & No requirement & No & No & 24 months & $\begin{array}{l}\text { Yes, period varies } \\
\text { and depends on } \\
\text { diagnosis }\end{array}$ & $\begin{array}{l}\text { Individual } \\
\text { capacity }\end{array}$ & SI/state \\
\hline $\begin{array}{c}\text { Turkey }^{\mathrm{M}} \\
\text { (Non-EU, Low GDP) }\end{array}$ & $\begin{array}{l}\text { No } \\
\text { requirement }\end{array}$ & 120 months & No requirement & No & No & No requirement & 2 years & \begin{tabular}{|l} 
Individual \\
capacity
\end{tabular} & SI/state \\
\hline $\begin{array}{c}\text { Great Britain } \\
(\text { EU-15, High GDP) }\end{array}$ & $\begin{array}{l}\text { No } \\
\text { requirement }\end{array}$ & No requirement & Yes $^{4}$ & No & No & 28 weeks & $\begin{array}{l}\text { Yes, period varies } \\
\text { and depends on } \\
\text { diagnosis }\end{array}$ & $\begin{array}{l}\text { Individual } \\
\text { capacity }\end{array}$ & SI/state \\
\hline $\begin{array}{c}\text { Ukraine }^{\mathrm{P}} \\
\text { (Non-EU, Low GDP) }\end{array}$ & $\begin{array}{l}\text { No } \\
\text { requirement }\end{array}$ & No requirement & No requirement & No & No & 3 months & $\begin{array}{l}\text { Yes, every } 12 \\
\text { months }\end{array}$ & $\begin{array}{l}\text { Individual } \\
\text { capacity }\end{array}$ & SI/state \\
\hline
\end{tabular}
A - Anglo-saxon, S - Scandinavian, B - Bismarkian, M - Mediterranean, P - Post-communist

EU - European Union, GDP - Gross Domestic Product (classification based on GDP per capita in international dollars), SI - social insurance

1 1501,82 / 1082,65 euro per trimester for a person $>=21 /<21$ years old, resp

$2>=36$ months of payments to the statutory pension insurance within the last 5 years

3 from $20-26$ y.o. depending on education

${ }^{4}$ depends on multiple factors of individual situation (online calculator)

${ }^{5}$ Public benefit: continuous employment, the last 26 weeks (min. 240 hours) Employer-paid benefit: 8 weeks of employment (min. 74 hours)

${ }^{6}$ Previously earned income 19,142.9 euro for invalidity pension / 9,482.16 euro for disabled adult allowance

7800 working hours in previous 12 months for invalidity pension

${ }^{8}>=36$ months of payments to the statutory pension insurance within the last 5 years 


\section{Online Supplementary Table S3. Regulations to prevent work disability and help staying at work for people with RA}

\begin{tabular}{|c|c|c|c|c|c|c|c|c|c|c|c|c|}
\hline \multirow[b]{2}{*}{ Country } & \multicolumn{8}{|c|}{ Facilities available to support stay at work } & \multirow[b]{2}{*}{\begin{tabular}{|} 
Participatio \\
$\mathrm{n}$ in \\
programs \\
to facilitate \\
employmen \\
t obligatory \\
before a \\
WD can be \\
granted?
\end{tabular}} & \multicolumn{2}{|c|}{ Working with disability } & \multirow[b]{2}{*}{$\begin{array}{l}\text { Forms of societal support for employment } \\
\text { of people with disabilities }\end{array}$} \\
\hline & $\begin{array}{c}\text { Yes/N } \\
\mathbf{0}\end{array}$ & $\begin{array}{c}\text { Vocati } \\
\text { onal } \\
\text { rehabi } \\
\text { litatio } \\
\mathrm{n} \text { in } \\
\text { the } \\
\text { hospit } \\
\text { al }\end{array}$ & $\begin{array}{c}\text { Vocatio } \\
\text { nal } \\
\text { rehabilit } \\
\text { ation in } \\
\text { private } \\
\text { sector }\end{array}$ & $\begin{array}{c}\text { Self- } \\
\text { learning } \\
\text { /self- } \\
\text { manage } \\
\text { ment }\end{array}$ & $\begin{array}{c}\text { Work } \\
\text { coaches }\end{array}$ & \begin{tabular}{|} 
Advice \\
from \\
occupati \\
onal \\
physicia \\
$n$
\end{tabular} & $\begin{array}{l}\text { Coaching } \\
\text { (advice) } \\
\text { from } \\
\text { employm } \\
\text { ent } \\
\text { agency }\end{array}$ & Other & & $\begin{array}{c}\text { Can a } \\
\text { person } \\
\text { with WD } \\
\text { perform a } \\
\text { paid } \\
\text { work? }\end{array}$ & $\begin{array}{l}\text { Does the } \\
\text { right to } \\
\text { work } \\
\text { depend on } \\
\text { degree of } \\
\text { disability? }\end{array}$ & \\
\hline $\begin{array}{c}\text { Albania }^{\mathrm{P}} \\
\text { (Non-EU, Low GDP) }\end{array}$ & Yes & Yes & No & Yes & No & No & No & & No & Yes & Yes & reductions in taxes for employers \\
\hline $\begin{array}{c}\text { Austria }^{\mathrm{B}} \\
\text { (EU-15_High GDP) }\end{array}$ & Yes & No & Yes & No & No & Yes & No & 8 & Yes & Yes $^{6}$ & No & $\begin{array}{l}\text { protection against dismissal; wage protection; } \\
\text { additional annual leave; benefits for the } \\
\text { employer and his business if disabled people } \\
\text { are employed; tax benefits }\end{array}$ \\
\hline $\begin{array}{c}\text { Belarus }^{\mathrm{P}} \\
\text { (Non-EU, Low GDP) }\end{array}$ & Yes & Yes & No & Yes & No & No & No & & No & Yes & Yes & reductions in taxes for employers \\
\hline $\begin{array}{c}\text { Belgium }^{\mathrm{B}} \\
\text { (EU-15, High GDP) }\end{array}$ & Yes & Yes & No & No & Yes & Yes & Yes & Yes $^{1}$ & No & Yes & Yes & $\begin{array}{l}\text { compensation for work place adjustments, } \\
\text { travel expenses, subsidies for employers }\end{array}$ \\
\hline $\begin{array}{c}\text { Bosnia\&Herzegovina } \\
\text { (Non-EU, Low GDP) }\end{array}$ & No & na & na & na & na & na & na & & No & No & NA & none \\
\hline $\begin{array}{c}\text { Bulgaria }^{\mathrm{P}} \\
\text { (EU-28_Low GDP) }\end{array}$ & Yes & Yes & No & Yes & No & Yes & Yes & & No & Yes & No & $\begin{array}{l}\text { obligatory quotas of patients with disabilities } \\
\text { that should be employed. }\end{array}$ \\
\hline $\begin{array}{c}\text { Croatia }^{\mathrm{P}} \\
\text { (EU-28, Low GDP) }\end{array}$ & Yes & No & No & No & No & Yes & Yes & Yes $^{2}$ & No & Yes $^{7}$ & No & $\begin{array}{l}\text { obligatory quotas of patients with disabilities } \\
\text { that should be employed. }\end{array}$ \\
\hline $\begin{array}{c}\text { Cyprus }^{\mathrm{M}} \\
\text { (EU-28, HighGDP) }\end{array}$ & Yes & Yes & Yes & Yes & Yes & Yes & Yes & & No & Yes & Yes & $\begin{array}{l}\text { obligatory quotas of patients with disabilities } \\
\text { that should be employed, subsidies for } \\
\text { companies, programs of additional education } \\
\text { and training }\end{array}$ \\
\hline $\begin{array}{l}\text { Czech Republic }^{P} \\
\text { (EU-28, High GDP) }\end{array}$ & Yes & Yes & Yes & Yes & No & Yes & No & & No & Yes & No & reductions in taxes for employers \\
\hline $\begin{array}{c}\text { Denmark }^{\mathrm{S}} \\
\text { (EU-15, High GDP) }\end{array}$ & Yes & Yes & Yes & Yes & Yes & Yes & Yes & & Yes & Yes & Yes & wages subsidies \\
\hline $\begin{array}{c}\text { Eestonia }^{\mathrm{P}} \\
\text { (EU-28, Low GDP) }\end{array}$ & No & na & na & na & na & na & na & & No & Yes & No & reductions in taxes for employers \\
\hline $\begin{array}{c}\text { Finland } \\
\text { (EU-15, High GDP) }\end{array}$ & Yes & Yes & Yes & Yes & No & Yes & Yes & & Yes & Yes & Yes & $\begin{array}{l}\text { subsidies for companies, programs of } \\
\text { additional education and training }\end{array}$ \\
\hline
\end{tabular}




\begin{tabular}{|c|c|c|c|c|c|c|c|c|c|c|c|c|}
\hline $\begin{array}{c}\text { France }^{\mathrm{B}} \\
\text { (EU-15, High GDP) }\end{array}$ & No & na & na & na & na & na & na & & No & Yes & No & $\begin{array}{l}\text { obligatory quotas of patients with disabilities } \\
\text { that should be employed. }\end{array}$ \\
\hline $\begin{array}{c}\text { Georgia }^{\mathrm{P}} \\
\text { (Non-EU, Low GDP) }\end{array}$ & No & na & na & na & na & na & na & & $\mathrm{n} / \mathrm{a}$ & na & na & none \\
\hline $\begin{array}{c}\text { Germany } \\
\text { (EU-15, High GDP) }\end{array}$ & Yes & No & Yes & Yes & Yes & Yes & Yes & & No & Yes $^{6}$ & Yes & $\begin{array}{l}\text { obligatory quotas of patients with disabilities } \\
\text { that should be employed. }\end{array}$ \\
\hline $\begin{array}{c}\text { Greece }^{\mathrm{M}} \\
\text { (EU-15, Low GDP) }\end{array}$ & No & na & na & na & na & na & na & & No & $\mathrm{Yes}^{7}$ & Yes & none \\
\hline $\begin{array}{c}\text { Hungary }^{p} \\
\text { (EU-28, Low GDP) }\end{array}$ & No & na & na & na & na & na & na & & No & Yes & Yes & $\begin{array}{l}\text { obligatory quotas of patients with disabilities } \\
\text { that should be employed. }\end{array}$ \\
\hline $\begin{array}{c}\text { Iceland }^{\mathrm{S}} \\
\text { (Non-EU, High GDP) }\end{array}$ & Yes & Yes & Yes & & & Yes & & & No & Yes & No & subsidies for companies \\
\hline $\begin{array}{c}\text { Ireland }{ }^{A} \\
\text { (EU-15, High GDP) }\end{array}$ & Yes & No & Yes & Yes & No & Yes & No & & No & Yes & Yes & $\begin{array}{l}\text { obligatory quotas of patients with disabilities } \\
\text { that should be employed, subsidies for } \\
\text { companies, workplace adaptations }\end{array}$ \\
\hline $\begin{array}{c}\text { Israel }^{\mathrm{B}} \\
\text { (Non-EU, High GDP) }\end{array}$ & Yes & Yes & Yes & No & No & Yes & No & & No & Yes & No & education programs \\
\hline $\begin{array}{c}\text { ItalyM } \\
\text { (EU-15, High GDP) } \\
\end{array}$ & Yes & No & No & Yes & No & No & No & & No & Yes & Yes & $\begin{array}{l}\text { obligatory quotas of patients with disabilities } \\
\text { that should be employedYes }\end{array}$ \\
\hline $\begin{array}{c}\text { Latvia }^{\mathrm{P}} \\
\text { (EU-28, Low GDP) }\end{array}$ & No & na & na & na & na & na & na & & No & Yes & No & subsidies for companies \\
\hline $\begin{array}{c}\text { Lithuania }^{\mathrm{P}} \\
\text { (EU-28, Low GDP) }\end{array}$ & Yes & Yes & Yes & Yes & & Yes & Yes & 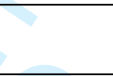 & Yes & Yes & Yes & Education programs \\
\hline $\begin{array}{c}\text { Luxemburg }^{\text {B }} \\
\text { (EU-15, High GDP) }\end{array}$ & Yes & No & No & No & No & Yes & No & Yes $^{2}$ & No & Yes & No & yes, details not specified \\
\hline $\begin{array}{l}\text { FYR Macedonia }{ }^{P} \\
\text { (Non-EU, Low) }\end{array}$ & Yes & Yes & Yes & Yes & No & Yes & No & & No & No & NA & $\begin{array}{l}\text { reductions in taxes for employers ( } 1 \text { year of } \\
\text { employment) }\end{array}$ \\
\hline $\begin{array}{c}\text { MaltaM } \\
\text { (EU-28, High GDP) }\end{array}$ & No & na & na & na & na & na & na & & No & Yes & No & $\begin{array}{l}\text { reductions in taxes for employers, subsidies } \\
\text { for companies, obligatory quotas of patients } \\
\text { with disabilities that should be employed }\end{array}$ \\
\hline $\begin{array}{c}\text { Moldova }^{\mathrm{P}} \\
\text { (Non-EU, Low GDP) }\end{array}$ & Yes & Yes & Yes & Yes & No & No & Yes & & Yes & Yes & Yes & $\begin{array}{l}\text { obligatory quotas of patients with disabilities } \\
\text { that should be employed }\end{array}$ \\
\hline $\begin{array}{c}\text { Montenegro } \\
\text { (Non-EU, Low GDP) } \\
\end{array}$ & Yes & Yes & No & No & No & No & No & & Yes & Yes & Yes & yes, details not specified \\
\hline $\begin{array}{c}\text { Norway } \\
\text { (Non-EU, High GDP) }\end{array}$ & Yes & yes & No & No & No & Yes & yes & & Yes & $\begin{array}{l}\text { Yes }(\text { after } \\
1 \text { year) }\end{array}$ & No & $\begin{array}{l}\text { in case of re-entering employment, employer } \\
\text { may for the first months (up to one year) get } \\
\text { refund of salary by the social security system. }\end{array}$ \\
\hline $\begin{array}{c}\text { PoLand } \\
\text { (EU-28, Low GDP) }\end{array}$ & Yes & Yes & No & Yes & Yes & Yes & Yes & Yes $^{4}$ & No & Yes & Yes & $\begin{array}{l}\text { obligatory quotas of patients with disabilities } \\
\text { that should be employed, educational } \\
\text { programs, subsidies for companies }\end{array}$ \\
\hline Romania $^{p}$ & Yes & No & No & No & No & Yes & No & & Yes & Yes & Yes & None \\
\hline
\end{tabular}




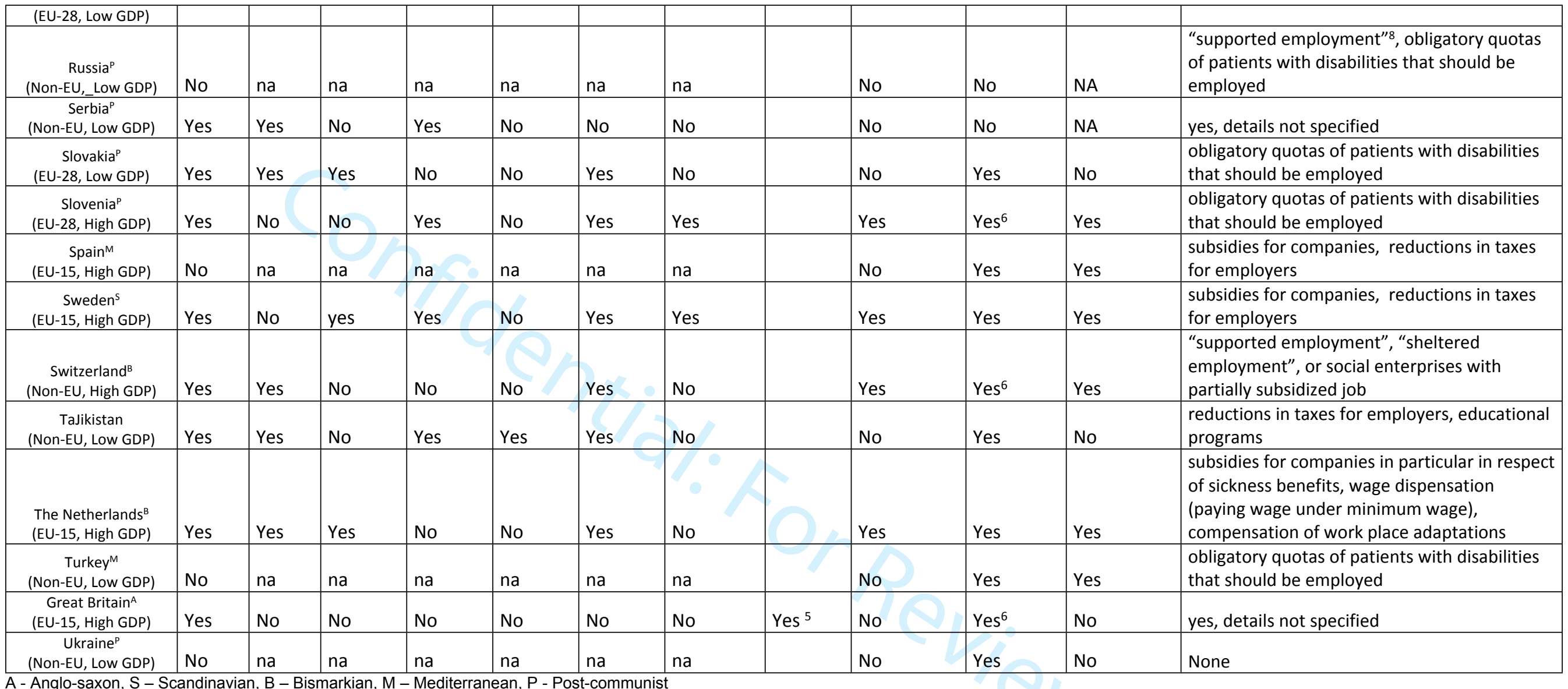

A - Anglo-saxon, S - Scandinavian, B - Bismarkian, M - Mediterranean, P - Post-communist
EU - European Union, GDP - Gross Domestic Product (classification based on GDP per capita in international dollars)

1 Progressive work reintegration

2 Professional rehabilitation right provided and payed by social insurance

3 Internal or external reclassification coached by social security

${ }^{4}$ Working in protected conditions, conditions of employment in sheltered workshops, customized workplace

${ }^{5}$ An occupational health assessment with recommendations made to their employer and doctor

6 With a limitation on earnings

${ }^{7}$ Amount of pension may be reduced

8 Salaries may be lower than average disability pension 


\begin{tabular}{|c|c|c|}
\hline 3 & & Total \\
\hline \multicolumn{3}{|c|}{ Rheumatologists characteristics (Questionnaire 2) } \\
\hline & & $539(33)$ \\
\hline & & $\underline{47.5(10.2)}$ \\
\hline & & $284(53 \%)$ \\
\hline \multicolumn{3}{|c|}{ Current work environment } \\
\hline 10 & $\underline{\text { Non-university hospital }}{ }^{* *}$ & $142(26 \%)$ \\
\hline 1 & University hospital & $278(51 \%)$ \\
\hline 12 & Private practice & $\underline{52(10 \%)}$ \\
\hline 13 & Other & $22(4 \%)$ \\
\hline \multicolumn{2}{|c|}{14 Years of work experience } & $2.5(1.1)$ \\
\hline \multicolumn{2}{|c|}{$\sqrt{\text { Average hours of patient care per week }}$} & $33.5(12.3)$ \\
\hline \multicolumn{3}{|c|}{16 Patient characteristics (Questionnaire 3) } \\
\hline \multicolumn{2}{|c|}{$\mathrm{N}$ of patients ( $\mathrm{N}$ of countries) } & $\underline{719(34)}$ \\
\hline \multicolumn{2}{|c|}{ Age (years) } & $\underline{53.1(12.2)}$ \\
\hline \multicolumn{2}{|c|}{20 Gender, female } & $541(76 \%)$ \\
\hline \multicolumn{2}{|c|}{21 Education } & \\
\hline \multirow{3}{*}{$\begin{array}{l}22 \\
23\end{array}$} & Primary & $110(16 \%)$ \\
\hline & $\underline{\text { Secondary }}$ & $254(36 \%)$ \\
\hline & $\underline{\text { High }}$ & $\underline{339(48 \%)}$ \\
\hline \multicolumn{3}{|c|}{25 Work status } \\
\hline \multicolumn{2}{|r|}{ Paid work } & $317(45 \%)$ \\
\hline & No paid work* & $180(26 \%)$ \\
\hline \multirow{2}{*}{4} & Work disabled (WD) & $172(24 \%)$ \\
\hline & Paid work + WD & $\underline{34(5 \%)}$ \\
\hline & & $15.0(11.1)$ \\
\hline & & $472(69 \%)$ \\
\hline
\end{tabular}

33 Values reflect mean (SD) or n (\%), as appropriate; WD - work disability * but not work disabled ** private or public Anglo-saxon: UK, Ireland; Scandinavian: Denmark, Iceland, Sweden,

34 Norway, Finland; Bismarkian: Austria, Belgium, Germany, France, Israel, Netherlands, Switzerland; Mediterranean: Cyprus, Greece, Italy, Portugal, Spain, Turkey; Post-communist: Albania

Bulgaria, Czech Republic, Croatia, Estonia, Georgia, Hungary, Latvia, Lithuania, Poland, Romania, Russian Federation, Serbia, Tajikistan, Slovak Republic, Slovenia 
Online Supplementary Table S4S5. Patients' and rheumatologists' perceptions across several domains according to (1) GDP per capita PPP (2) EU membership status (3) Type of social security system

\begin{tabular}{|c|c|c|c|c|c|}
\hline & \multicolumn{3}{|c|}{ Patients' perceptions $\xi$} & \multicolumn{2}{|c|}{ Rheumatologists' perceptions ${ }^{\xi}$} \\
\hline & $\begin{array}{l}\text { Importance and } \\
\text { support to remain } \\
\text { employed }(0-5) \\
\mathrm{n}=520\end{array}$ & $\begin{array}{l}\text { Process of } \\
\text { applying for work } \\
\text { disability pension } \\
(0-4) \\
n=360\end{array}$ & $\begin{array}{l}\text { Obtaining and } \\
\text { living with work } \\
\text { disability pension } \\
(0-6) \\
\mathrm{n}=358\end{array}$ & $\begin{array}{l}\text { Performance of the } \\
\text { system }(0-4) \\
\mathrm{n}=498\end{array}$ & $\begin{array}{l}\text { Role of the } \\
\text { rheumatologists } \\
(0-4) \\
n=507\end{array}$ \\
\hline $\begin{array}{l}\text { GDP per capita (int. \$) } \\
\text { High vs low GDP }\end{array}$ & $0.16[-0.12 ; 0.44]$ & $0.36[-0.27 ; 0.99]$ & $-0.13[-0.49 ; 0.23]$ & $0.31[-0.08 ; 0.69]$ & $-0.54[-0.89 ;-0.20]$ \\
\hline $\begin{array}{l}\text { EU membership } \\
\text { EU vs non-EU member }\end{array}$ & $0.19[-0.13 ; 0.52]$ & $-0.43[-1.17 ; 0.32]$ & $-0.19[-0.61 ; 0.24]$ & $0.02[-0.43 ; 0.46]$ & $-0.42[-0.83 ;-0.00]$ \\
\hline $\begin{array}{r}\text { Type of system } \\
\text { Scandinavian } \\
\text { Anglo-Saxon/liberal } \\
\text { Bismarckian/conservative } \\
\text { Mediterranean/southern } \\
\text { Post-Communist/eastern }\end{array}$ & $\begin{array}{l}\text { Reference } \\
-1.02[-1.62 ;-0.42] \\
-0.39[-0.88 ; 0.11] \\
-0.55[-1.04 ;-0.06] \\
-0.58[-1.00 ;-0.15]\end{array}$ & $\begin{array}{c}\text { Reference } \\
-1.87[-3.24 ;-0.50] \\
-1.02[-2.15 ; 0.12] \\
-1.37[-2.46 ;-0.29] \\
-1.14[-2.07 ;-0.22]\end{array}$ & $\begin{array}{c}\text { Reference } \\
-0.42[-1.25 ; 0.41] \\
-0.02[-0.71 ; 0.67] \\
-0.25[-0.90 ; 0.41] \\
-0.11[-0.67 ; 0.46]\end{array}$ & $\begin{array}{c}\text { Reference } \\
-1.40[-2.14 ;-0.67] \\
-0.29[-0.79 ; 0.21] \\
-1.18[-1.68 ;-0.67] \\
-0.86[-1.30 ;-0.42]\end{array}$ & $\begin{array}{c}\text { Reference } \\
-0.60[-1.47 ; 0.27] \\
-0.34[-0.95 ; 0.27] \\
-0.10[-0.71 ; 0.51] \\
0.24[-0.29 ; 0.77]\end{array}$ \\
\hline
\end{tabular}

Coefficients are derived from multilevel models (with individuals clustered in countries) and each independent variable (i.e. GDP, EU membership or type of system) entered in a separate model; GDP - Gross Domestic Product, EU - European Union

Anglo-saxon: UK, Ireland; Scandinavian: Denmark, Iceland, Sweden, Norway, Finland; Bismarkian: Austria, Belgium, Germany, France, Israel, Netherlands, Switzerland; Mediterranean: Cyprus, Greece, Italy, Portugal, Spain, Turkey; Post-communist: Albania, Bulgaria, Czech Republic, Croatia, Estonia, Georgia, Hungary, Latvia, Lithuania, Poland, Romania, Russian Federation, Serbia, Tajikistan, Slovak Republic, Slovenia

§the higher score the more positive are the perception 
Online Supplementary Table S5S6. N(\%) of missing data per variable.

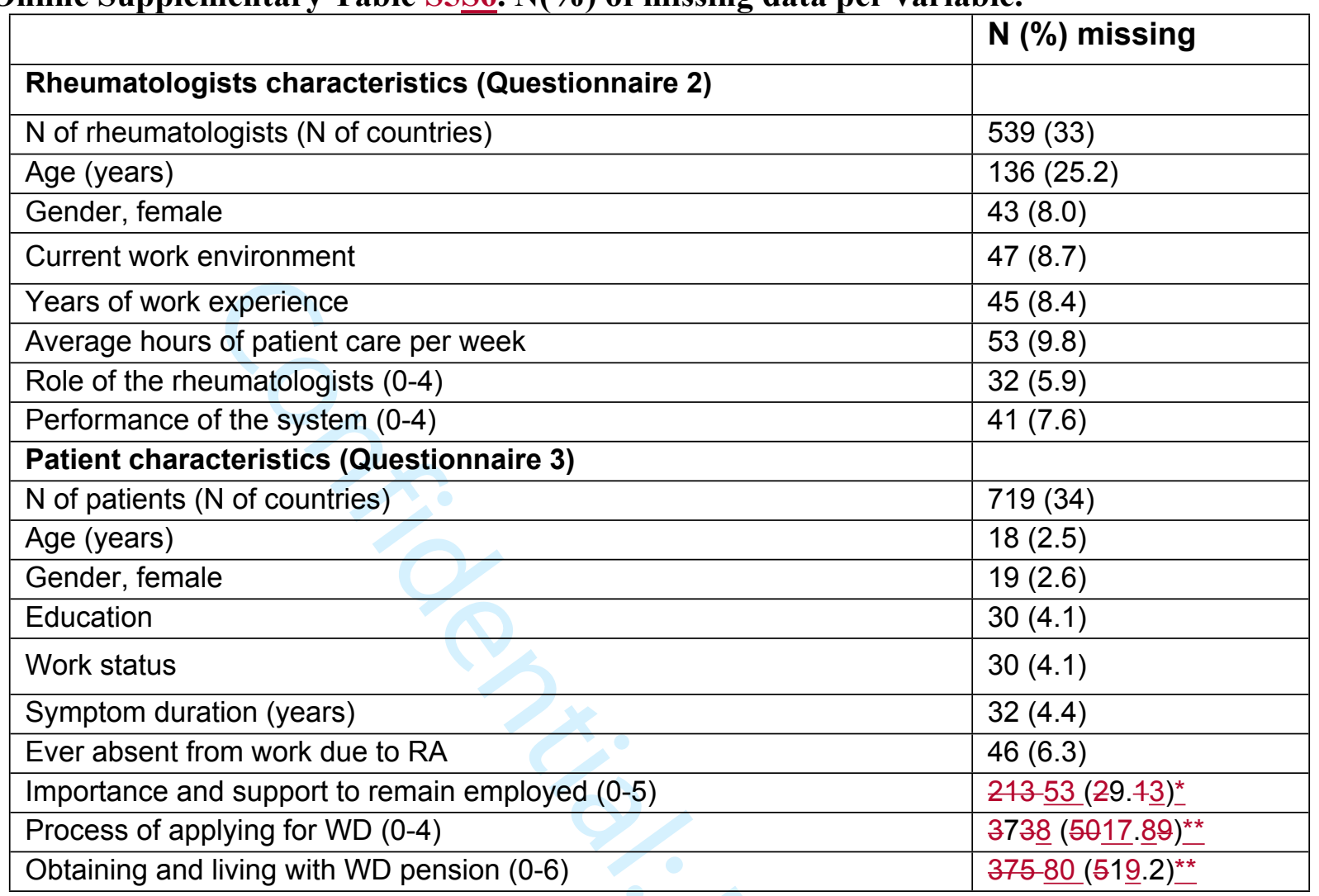

WD - work disability * but not work disabled ${ }^{* *}$ private or public

*only assessed in patients currently or ever having worked $(n=573)$

**only assessed in patients currently work disabled or ever considered applying for WD $(n=438)$ 


\section{Part I: Formal regulations and rules}

- One investigator per country filled in the questionnaire

\section{Part II: survey among rheumatologists}

- >15 rheumatologists per country on perceptions of social security system

\section{Part III: survey among patients}

- > 15 patients per country on perceptions of social security system

Figure 1. Study design. 
FORMATTING AMENDMENTS (if any)

Required amendments will be listed here; please include these changes in your revised version:

Please make sure the following statements are included in the main document file, which should match the details given in the submission pages:

Competing interests

Contributorship

Acknowledgements

Funding info

Patient and Public Involvement

Ethical approval information

Data sharing statement

\section{Authors' answer: The requested statements have been added at the end of the} manuscript.

The figure(s) shouldn't be embedded in the text file, but supplied in 300dpi TIFF, EPS, JPEG or PDF format. And legend(s) supplied at the end of the manuscript.

Authors' answer: The figure has now been uploaded separately, a legend is included at the end of the manuscript.

Please modify the references according the guidelines:

1 (list 3 authors et al if there are or fewer) Surname AB, Surname CD. Article title.

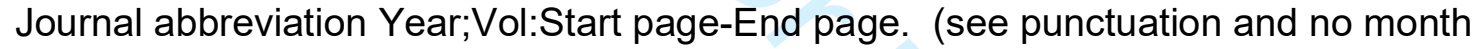
after year of publication)

See: https://authors.bmi.com/writing-and-formatting/formatting-your-paper/

Authors' answer: The reference style has been amended as per requirements.

If your article is a Clinical trial, please comply with our new format requirements for Clinical trials, including extension studies.

See: http://ard.bmj.com/pages/authors/ under the heading Clinical trials, including extension studies

Authors' answer: Our study does not report on a clinical trial. 
Authors' answer to reviewer 1: The authors appreciate the critical reflection of the reviewer and appreciation of the complexity of the studied topic. Indeed, recourse to (official) disability is a complex construct in which social security has a limited role, and our study reinforced this.

The idea of clinical vignettes is valuable and was indeed discussed by our research team at the start of the project. Given the paucity of studies in this field we aimed first to sketch the broad picture of the state of art, and therefore we have opted for the questionnaire approach to review rules and regulations as well as perceptions thereof. We are pleased to have the opportunity to clarify that we have still used vignettes for collection of part of data on income compensation using the 9 scenarios for a female with 25 years of full-time work history: 1) sick leave for 6 months 2) long-term work disability in case of $50 \%$ incapacity to work 3 ) long-term work disability in case of $75 \%$ incapacity to work, and each scenario considered for income at the level of minimum salary, average salary and 3 times average salary. The vignettes were initially referred to as 'scenarios' on p. 6 of the manuscript. This has now been clarified in the manuscript, and vignette template also added to Online Appendix Text S1b.

Clinical vignettes to extensively study application of regulations and judgments whether a work disability would be the 'preferred/best' solution given patient's health and his/her personal and environmental context would be a logical follow up of our study, which outlined some major differences between the countries and in this ways informs further research activities. This was also a next planned step we had, admittedly not mentioned in the discussion, which would probably benefit from it. 
We have edited part of the methods (p. 6) and discussion (p. 17) which now read as follows:

p. 6: The questionnaire for Pls addressed national regulations (in 2014) on benefits separately for sick leave and WD (Online Supplementary Text S1a: availability and affordability), as well as calculations of the level of income for nine pre-specified scenarios (vignettes), one on sick leave and two for long-term WD across the three levels of income (Online Supplementary Text S1b). In countries where a patient research partner was available $(n=21)$, he/she was invited to comment on any inconsistencies in the answers on the main questionnaire about the formal regulations. In this case, answers were double-checked with the PI.

p. 17: Recourse to (official) disability is a complex construct in which social security has a limited role. Alternative approaches to gain insight into international variation should be considered to study the performance and impact or social security systems. One of the potentially promising method could consist of series of clinical vignettes. By considering the rules and regulations that are to be applied to a hypothetical patient with given characteristics in terms of disease, work situation and disability, as well as attitudes and values about role of work in life and society, countries could be compared and further classified.

\section{Reviewer: 2}

Comments to the Author

The topic of work participation and work disability compensation is an important one and you are to be commended for a systematic study of the phenomena. The main issue for this reviewer is the generalizability of the sampling of primary rheumatologist in each country as well as the resulting samples of about 15 rheumatologists and patients per country. In short, how was the primary reporting rheumatologist selected in each country? While well known rheumatologists and/or those with high patient loads may be able to report on country compensation systems, they may still not account for the bulk of RA patients and so may have a unrepresentative picture of the reality of the patients in their respective country. Similarly, their selection of the rheumatologists and patients for the more impressionistic study questionnaires could be problematic. At the very least, how the primary rheumatologists and other participants were enrolled should be given adequate description. it would be preferable to at least attempt to show how representative they are of the universe of rheumatologists and RA patients in each country.

In the end, however, the most important issue is establishing the extent to which your informants among rheumatologists and patients are representative of the universe from which they were chosen.

The important data you report will be more influential once the reader has a sense of the context of the respondents relative to the sampling universes of rheumatologists and patients. 
Another issue is that, as of you note, official policy and unofficial bureaucratic norms may affect how the system is experienced. Providing some qualitative description of how the official policies and unofficial implementation differ may be necessary for the reader to know whether the impressions of the rheumatologists and patients reflect what's on paper or just how the policies are with real world implementation.

\section{Authors' answer to reviewer 2/question 2:}

We are pleased to hear that the reviewer shares our point of view on official and unofficial regulations. This aligns with the acknowledgement by reviewer 1 that 'official disability' is a social construct in which the role of the social security system is limited. In addition to the adjustment based on the comment of reviewer 1 , we have also expanded on this in the manuscript (p. 17) to make this point clear to the readership. This paragraph now reads as following:

We have to acknowledge that the actual application of the formal rules and regulations can differ substantially from the formal rules themselves, and hence patients in countries with similar rules could potentially have different experiences. For example, partial work disability (which formally implies that a patient can work part of the time) may be a barrier to any employment in some countries and thus perceived differently compared to countries where patients deemed partially disabled are able to use their right for part-time work. We have attempted to get the initial insight into this through the surveys among patients and rheumatologists.

\section{Reviewer: 3}

Comments to the Author This is an interesting study by Dr. Putrik and colleagues examining policies around sick leave and work disability for patients with RA across Europe. The strengths of

https://mc.manuscriptcentral.com/ard 
the study are the following: 1) this is a relatively understudied area and therefore the study and findings are novel; 2) the number of countries surveyed is quite comprehensive; 3) the variation in policies, although expected, is interesting. The weaknesses of the study are the following: 1) The reader gets lost in a large amount of data drawn from several surveys. This feeling of getting lost is at least in part because no hypotheses are stated in the introduction. What are 2-3 main hypotheses that you were trying to explore with this research? Without organizing the paper in this way, the paper reads like a descriptive thesis rather than hypothesis-driven scientific manuscript; 2) the number of patients from each country is likely too low to draw any reliable conclusions, particularly since not all were on work disability and given the very high missing item count for some of the survey items.

\section{Authors' answer to reviewer 3/question 1:}

We thank reviewer for appreciation of the strengths of our study. With respect to the mentioned limitations, we have addressed them and provide detailed answers to the specific comments point-by-point below.

Additional specific comments as follows:

-Introduction:

There needs to be a stronger justification for the study and if possible, some specific hypotheses listed. As is, the aim is just too broad, "To describe and explore differences in formal regulations around sick leave and WD" - what specific aspects were explored and why? That will go a long way in framing the paper and ensuring reader's don't get lost in a sea of data.

\section{Authors' answer to reviewer 3/question 2:}

To address the reviewers comments, authors revised the introduction and specified the hypotheses tested in this study (pp. 5-6). The edited part of the introduction now reads:

SS arrangements have been suggested to result in differences in overall patterns of employment, WD, and retirement.[13, 14] A recent worldwide multinational study (COMORA) has shown that lower economic wealth and human development of countries is associated with higher rates of unemployment and higher absenteeism. [15] Earlier, QUEST RA study has observed that in low Gross Domestic Product (GDP) countries, people remain working with higher levels of disability and disease activity compared to high GDP countries.[16] The regulations around the sick leave and work disability may be, at least partially, responsible for these differences, however, they have not been studied.

Policies with regard to work participation, however, go far beyond income substitution only. Criteria for access to WD benefits as well as levels of income substitution likely depend, among others, on economic, political and cultural factors. While the European Union (EU) and World Health Organization (WHO) accept the historical differences in the way health and social systems are organized and function in their member states, there is a universal agreement that differences should not result in inequalities in health and quality of life of people across nations. [17] It is unclear whether EU member states achieved any degree of homogeneity in the key regulations around social policies with

https://mc.manuscriptcentral.com/ard 
regards to work disability. In addition to system level factors, there is evidence that personal contextual and disease related factors [6, 18, 19], influence the decision of an individual to take sick leave or apply for long term WD.

The aim of this study was to describe and explore differences in formal regulations around sick leave and WD for patients with RA, as well as perceptions by rheumatologists and patients on the system's performance, across European countries. We hypothesized that 1) lower GDP countries have stricter rules with regard to obtaining work disability and lower income substitution once work disability is granted

2) EU countries have more homogeneous regulations compared to non-EU countries 3) patients and rheumatologists in high GDP and EU member states are more satisfied with the performance of the social security system.

\begin{abstract}
-Methods:
--Can you please provide more information on the IRB procedure? Were local IRBs required before surveying patients at individual sites, or was there a central IRB?
\end{abstract}

\begin{abstract}
Authors' answer to reviewer 3/question 3:
\end{abstract}
Before the start of the study, we have obtained the ethical clearance from Maastricht Ethical Review board. We have shared this document with the Principal Investigators in each country. It was the responsibility of the Principal Investigator to adhere to local IRB procedures. In some countries, the decision of Maastricht IRB was sufficient, in other countries the PI had to go through an additional local procedure. We have now clarified this in the relevant section at the end of the manuscript.

Ethical approval information

The project has been approved by the Ethical Committee from the Maastricht University Medical Center. Principal Investigators in each country were responsible for local ethical approvals, where necessary.

\begin{abstract}
--Can you clarify why you went to rheumatologists for information on sick leave and WD benefits (rather than, for example, government agencies who might provide formal policies)? In the U.S., the average rheumatologist doesn't know the details of these policies - it may be different in Europe. Although the large percentage of missing variables in Supp Table S5 (51\% for process of applying for WD and $51 \%$ for obtaining and living with WD), seems to support this.
\end{abstract}

Authors' answer to reviewer 3/question 4:

The reviewer definitely raises a relevant point. When developing the study protocol, authors were aware that governmental authorities would more likely be able to provide data on regulatory procedures around sick leave and work disability.

However, the feasibility of this approach is very limited (e.g. language issues but also motivation to collaborate in research) and we have opted to rely on our established international network of rheumatologists. We strongly encouraged rheumatologists to refer to local agencies for information, and to our knowledge, most of them did so. This was a compromise solution to ensure we cover a large amount of countries, 
which is, as also acknowledged by the reviewer, one of the major strengths of this study. We emphasize in the discussion and limitations (p. 17) that this is an exploratory study which can only provide a first insight in this understudied topic. The edited text reads as follows:

While government authorities would be the most knowledgeable parties to enquire about rules and regulations, the feasibility issues around establishing a direct contact with agencies from >30 European countries, in different languages, and where they likely have little or no interest to contribute to a research project, led us to choose for the well-established network of rheumatologists active in research was approached instead. To improve data quality and accuracy, Pls were encouraged to seek help from other experts in their country; the summary was sent to available patient partners for a face-validity check of data in their country, and the results showed a good agreement. It is emphasized in the discussion that this study has an explorative nature and limited conclusions should be drawn.

--Can you clarify why you included patients who had not applied for WD - how would these patients know about regulations that do not apply to them?

Authors' answer to reviewer 3/question 5:

Patients who have not (yet) apply for work disability may be an important source of information about their perceptions of the system. Some may have tried to apply and have been unsuccessful, others never applied because of - perceived - system barriers. To account for prior knowledge and relevance of the questions for the patients, we have limited the analyses in the following way (as described on p. 8):

- 'Importance and support to remain employed' was assessed in patients currently or ever having worked.

- 'Process of applying for work disability' and 'Obtaining and living with work disability' were limited to patients currently work disabled or ever considered applying for WD. This also explains apparent large number of reported missing data which is further address in the answer to question 13.

--The translation procedures for the patient questionnaire are likely inadequate (having the PI translate the questionnaires). Generally, at the very least, a backtranslation to English by a different person to ensure the meaning is not lost would be beneficial. I realize this is a limitation that cannot be addressed at this point.

Authors' answer to reviewer 3/question 6:

We agree with the reviewer that validated translation procedures would have made the translations more accurate. After initial translation by Pls, we have though asked local patient partners who were proficient in English language to verify the translations and reassuringly no major issues were raised for the questionnaires that were checked. Therefore, while the translation procedures used remain a strong limitation (acknowledged in the discussion (p.17)), we have reasons to believe that translation was unlikely to cause serious bias in this exploratory analyses.

https://mc.manuscriptcentral.com/ard 
-In the methods, the authors state that they investigated whether regulations differ by the type of welfare regime, GDP, EU membership, country-level WD rates. That is a lot of comparisons and getting back to the introduction, there is really no justification of why these specific things were looked at, what the specific hypothesis were etc. For example, why would EU membership influence the examined relationships (assuming that the average reader does not know the details of EU policies and how they would relate to WD etc).

Authors' answer to reviewer 3/question 7:

This comment has now been addressed and introduction has been edited throughout (see Authors' answer to reviewer 3/question 2).

-The question where questionnaire item numbers "Q23" doesn't work. The average reader is not going to look at item-by-item questions in the supplement. I would recommend keeping this description at a high level and then referring to the supplement just once for more detail.

\title{
Authors' answer to reviewer 3/question 8:
}

We thank reviewer for this comment. We have edited the text and references to the online supplementary file pp.7-8 to facilitate readership. The paragraph now reads as follows:

\begin{abstract}
Answers of rheumatologists on their perspective of SS arrangements were summarized and scored (the higher is the better) around the two domains: (1) 'Performance of the system' (score 0-4) and (2) 'Role of the rheumatologists' (score $0-4)$. Additionally, a single item on the perceived standardization in the decisionmaking process was analyzed separately. Input from patients was summarized following three domains: (1) 'Importance and support to remain employed' (score 0-5), (2) 'Process of applying for WD (score 0-4), and (3) 'Obtaining and living with work disability pension' (score 0-6) (complete questionnaires are provided in the Online Supplementary Texts S2 and S3). Each domain consisted of 4 to 6 questions (each on a 1 (totally agree) to 5 (totally disagree) Likert scale, dichotomized as 1 ('(totally) agree') and 0 ('not agree/not disagree', '(totally) disagree'). The dichotomized scores per question were summed into the five domain scores (two for rheumatologists and three for patients).
\end{abstract}

\begin{abstract}
-Again, with the theme of too many comparisons being made - the authors examine the relationship between rheumatologists age, gender and work setting on preceptions. This almost feels like a whole other paper (shift from policies and country-level things to individual characteristics). I really think it is just too much for one paper. Similarly, for patients, why would a patient's gender/age/earning capacity influence their perceptions - although these factors may certainly play a role, the paper does not set the reader up to understand the importance of making such a comparison or justify why this is being explored.
\end{abstract}


Authors' answer to reviewer 3/question 9:

We appreciate the comment. We have initially considered two publications but preferred to avoid multiplying the papers for the same project, a 'slicing' phenomenon not appreciate in the scientific community. We would like to emphasize that individual characteristics of patients and rheumatologists are only used to adjust regression analyses and the associations between the individual factors and perceptions about social security system are not interpreted or discussed separately. The main research question and interpretation from the models remains the effect of countrylevel socio-economic factors (e.g. GDP, EU-membership, social security system) on the perceptions about the social security system. To reduce unessential details and simplify the paper, we have now moved descriptive statistics of the individual characteristics from Table 3 to Online Supplementary Table S4.

\section{Results:}

-The sentence, "Remarkably, multilevel analysis revealed that rheumatologists in high GDP and EU member countries felt less confident in having an active role in WD decisions". I am not sure why this is "remarkable" - isn't this expected? In countries with more resources, there is often a larger set of resources to address these issues - for example, there might be more infrastructure in primary care settings or more government agencies. This may then place less burden on the rheumatologists. Even if this is not the case, further clarification of this point is needed.

Authors' answer to reviewer 3/question 10:

Authors thank reviewer for this insight. We agree with the comment and have downtuned the statement towards a more constructive reflection. The edited sentence in the Results (p. 9-10) now reads as follows:

Multilevel analyses revealed that rheumatologists in high GDP (vs low GDP) and EUmember (vs non-EU member) countries felt less confident in having an active role in $W D$ decisions $(\beta=-0.5[95 \% \mathrm{Cl}[-0.9 ;-0.2]$ and $\beta=-0.5$ [-1.0;-0.1], respectively).

And pp. 16-17 in the Discussion:

At the same time, little differences were related to country's wealth or EUmembership, and only a weak signal suggested that rheumatologists in lower income countries are more confident in their role to support patient in work disability issues. The latter may indicate that rheumatologists in low income countries interact more intensively with patients on these issues and accept work disability questions as part of their responsibility. Alternatively, other cultural and system factors could be considered, e.g. patients referring to other professionals within the system (such as primary care or state agencies) for advice and support regarding work disability decisions.[26]

-The Supplemental Tables are so extensive - I suspect if the paper is more focused, at least half of these Tables can be removed.

Authors' answer to reviewer 3/question 11:

https://mc.manuscriptcentral.com/ard 
-Please include a more detailed discussion of missingness. There are survey items that have over $50 \%$ missing and this is a significant limitation that needs to be addressed directly.

\section{Authors' answer to reviewer 3/question 13:}

We thank reviewer for this comment that allowed us to spot inaccuracy of reporting the numbers of missing data. The apparently large number of missings on the domains 'Process of applying for work disability' (50.9\%) and 'Obtaining and living with work disability' $(51.2 \%)$ - which are combinations of the survey items as outlines on p.7 of the manuscript (composition and calculation of the domains) - is explained by the fact that analyses were limited to patients currently work disabled or ever considered applying for WD. To prevent misleading interpretations when calculating $\mathrm{N}$ of missing from the total number of patients, we have now edited the table S6 in the appendix to make this point clear. We hope this clarifies the issue and reassures the reviewer the missingness, albeit present as in almost any survey research, does not present a major concern. 